\title{
Quantification of Potential Ecological Impacts of Road Transport
}

\author{
Dissertation \\ in order to acquire the doctoral degree \\ from the Faculty of Economic Sciences \\ at the Georg-August-Universität Göttingen
}

Submitted by

Jan Friedrich

Born in: Bamberg

Göttingen, 2014 



\title{
Quantification of Potential Ecological Impacts of Road Transport
}

\author{
Dissertation \\ in order to acquire the doctoral degree \\ from the Faculty of Economic Sciences \\ at the Georg-August-Universität Göttingen
}

Submitted by

Jan Friedrich

Born in: Bamberg

Göttingen, 2014 
Thesis Committee

First academic advisor: Prof. Dr. Jutta Geldermann

Other academic advisors: Prof. Dr. Christina von Haaren

Prof. Dr. Oliver Mußhoff

Other members of the examination board:

Prof. Dr. Lutz Kolbe

Date of oral examination: June $16^{\text {th }} 2014$ 




\section{Acknowledgements}

I thank the sun, the rain, the birds, the trees, my friends, my family, my academic advisors, music, tennis and all people, places and beings around the world, and here particularly those whom I have come to meet, for their beauty, sustenance, support of life, help and friendship. 

Live simple so that other beings can simply live,

and you may become rich in friendship and fulfillment.

(Adapted from Mahatma Gandhi) 



\section{Table of Contents}

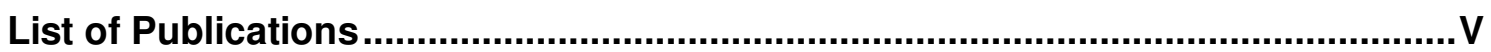

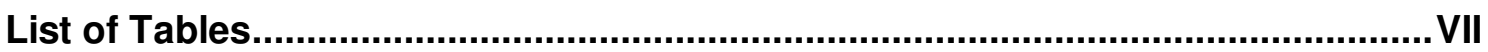

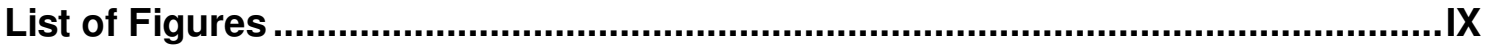

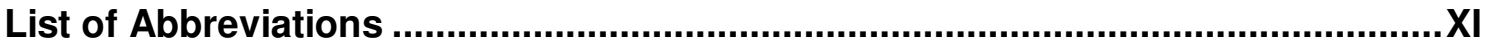

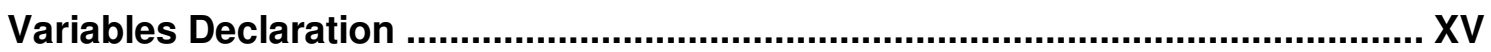

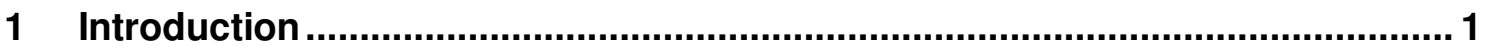

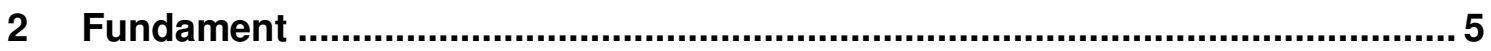

2.1 Groundwork of the Natural Sciences ............................................... 5

2.1.1 The Condition of Biological Diversity ................................................. 6

2.1.2 The Research Field 'Road Ecology' ................................................... 9

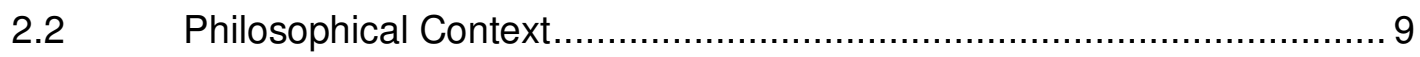

2.2.1 On the Relation of Humans and Non-Human Nature ........................ 10

2.2.2 Insights from Nussbaum's Capabilities Approach ........................... 13

2.2.3 Implications of Becker's Analysis of the Sustainable Person .............. 15

2.3 Conditions and Constraints of Sustainability in Society ..................... 17

2.3.1 The Term 'Sustainability' and Sustainable Conduct .......................... 18

2.3.2 Five Constraints to Sustainable Action in Business .......................... 19

2.3.3 The Concept of Degrowth .......................................................... 20

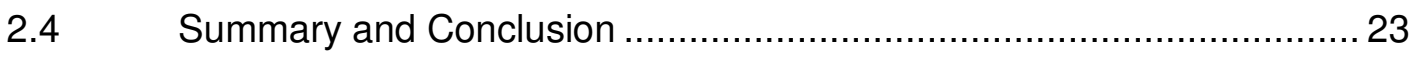

3 Methods Used in Environmental Management .......................................... 25

3.1 Corporate and Organizational Tools of Environmental Management: LCA, EMAS, ISO, and GRI 
3.2 Environmental Management Tools Used by Public Authorities: EIA and SEA, Ecological Risk-Analysis, the DPSIR-Model, and the Precautionary Principle 30

3.3 Aggregate Landscape Indicators of Land Use and Fragmentation ....... 34

S.4 Summary and Conclusion .......................................................... 40

4 Selected Ecological Impacts of Roads and Traffic ........................................ 43

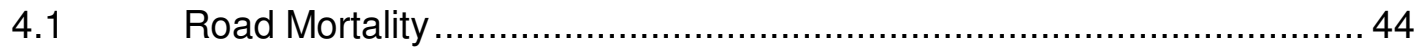

4.2 Loss and Degradation of Habitat ................................................ 46

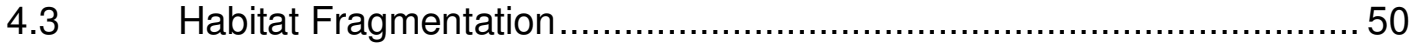

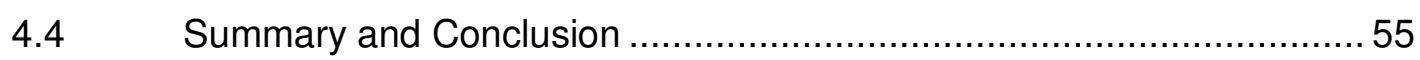

5 Complementing Environmental Indicators................................................... 57

5.1 Quantification of Land Use from Road Transport...............................57

5.1.1 Determination of the Share of Sealed Area of an Individual User....... 58

5.1.1.1 Measuring sealed area by the average width of road lanes .............58

5.1.1.2 Accounting for bypasses and resting areas ................................ 61

5.1.1.3 Mathematical representation and further input variables ................. 64

5.1.2 Determination of the Share of Degraded Area of an Individual User .. 68

5.1.2.1 The extension of sound pressure level from physical modeling........70

5.1.2.2 Determination of $55 \mathrm{~dB}(\mathrm{~A})$ as an animal disturbance threshold........ 74

5.1.2.3 Consideration of traffic volume variations within a 24 hour day ........ 77

5.1.2.4 Input data needed to calculate noise zone width........................... 79

5.1.2.5 Noise zone formulae derived from theoretical physical modeling ..... 80

5.1.2.6 Development of a correlation between noise zone width and traffic volume from a real data sample

5.1.2.7 Comparison of the two approaches pursued and development of a final methodology of quantification of impact. 88

5.1.2.8 Comparison of the results of the formula developed in this work to other results. 94

5.1.2.9 Further development of the interim method .98

5.1.2.10 Possibilities of impact mitigation 107 
5.2 Quantification of Fragmenting Impact from Road Transport .............. 107

5.2.1 Determination of the Fragmenting Impact of a Road User................ 108

5.2.1.1 Corridor- and prioritization-based quantification .......................... 109

5.2.1.2 Landscape discretionary quantification .................................... 118

5.3 Application of the Indicators ....................................................... 124

5.4 Remaining Limitations of the Indicators ..................................... 128

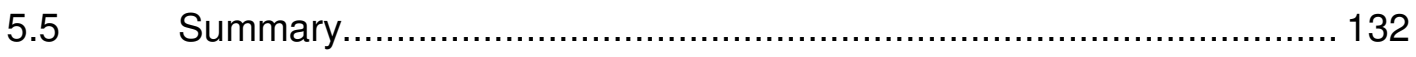

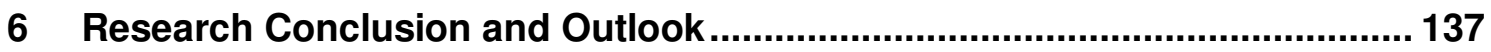

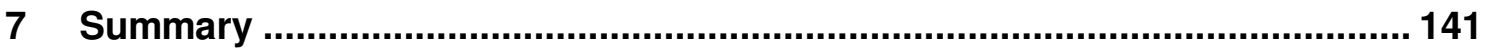

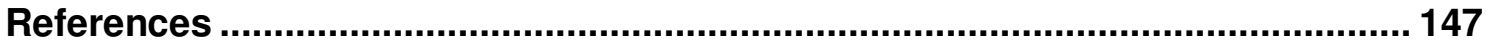

Appendix

A Discussion of the Meaning of Sustainability and of the Implications ... 171

B Five Economic Constraints to Sustainable Action - A More

Detailed Analysis................................................................... 174

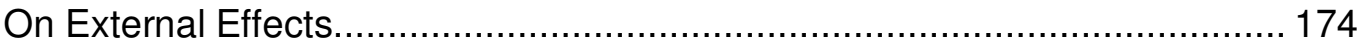

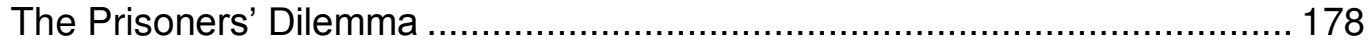

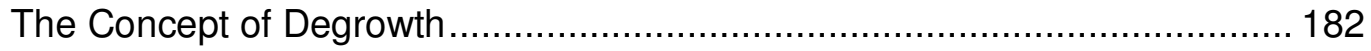

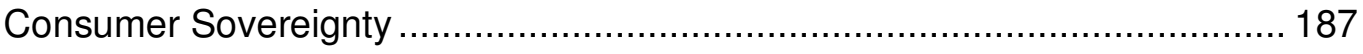

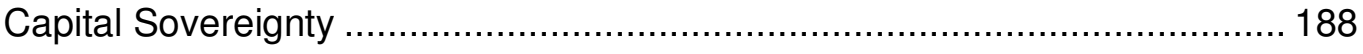

C Map of the Federal States of Germany.......................................... 190

D Traffic Volume Impacts on Wildlife at Different Thresholds................ 191

E Maps of the Two Exemplary Routes............................................ 192

Articles I - IV 



\section{List of Publications}

The dissertation consists of the present text and the following articles:

[ I ] Friedrich, J., and J. Geldermann. 2013a. Potentielle Auswirkungen von Straßenverkehrstransporten auf die Biodiversität. In Treffpunkt Biologische Vielfalt 12: Interdisziplinärer Forschungsaustausch im Rahmen des Übereinkommens über die Biologische Vielfalt, eds. U. Feit and H. Korn, pp. 107-113. Bonn: Federal Agency for Nature Conservation.

[ II ] Friedrich, J., and J. Geldermann. 2013b. Current practices of mitigating the ecologically harmful effects of roads - An assessment. In Societal dimensions of the conservation and utilization of biological diversity, eds. J. Friedrich, A. Halsband and L. Minkmar, pp. 341-358. Goettingen: Goettingen University Press.

[ III ] Friedrich, J., A. Henriquez Brash, M. Aguayo Arias, and J. Geldermann. Traffic Induced Landscape Fragmentation in the Biobío Region of Chile: Analysis and Prospects. Submitted October $21^{\text {st }} 2013$ to: International Journal for Social Ecology and Sustainable Development.

[ IV ] Friedrich, J. Integrating neglected ecological impacts of road transport into corporate management. Submitted June $10^{\text {th }} 2014$ to: Ecological Indicators. 



\section{List of Tables}

Table 5.1: $\quad$ Average width of lane representing the construction of the entire roadway per road category in meters.

Table 5.2: Length of federal highways and freeways per number of traffic lanes in $\mathrm{km}$.

Table 5.3: Absolute land use values (in $\mathrm{km}^{2}$ ) and proportions of resting areas 62

Table 5.4: $\quad$ Area sealed in Germany for roadways, bypasses, and resting areas in $\mathrm{km}^{2}$.

Table 5.5: Mileage shares and vehicle factors.

Table 5.6: Average values of hourly daily traffic volume (tvøh) and share of heavy duty vehicles $(p)$ for different types of roads and times during the day......

Table 5.7: Average shares of heavy duty vehicles in German freeways and federal highways.

Table 5.8: Decreasing accession of the noise zone per additional vehicle as of the formulae for both federal highways and freeways.

Table 5.9: Average extension of sound pressure levels $\geq 55 \mathrm{~dB}(A)$ in meters subject to average daily traffic volume yielded by the formulae of the theoretical approach.

Table 5.10: Average extension of sound pressure levels $\geq 55 \mathrm{~dB}(\mathrm{~A})$ in meters.

Table 5.11: Results of differing expressions of daily traffic volume and noise zone correlation.

Table 5.12: Noise zone expressions derived from the data sample changed to

$\mathrm{L}_{\text {DEN-energetic. }}$

Table 5.13: Display of the noise zone results using $L_{D E N-e n e r g e t i c}$ for the practical approach.

Table 5.14: Expansion of the noise zone assuming total attenuation values of $3.5,4$ and $6 \mathrm{~dB}(\mathrm{~A})$ per doubling of distance.

Table 5.15: Mileage shares and vehicle factors II. 104

Table 5.16: Number of conflicts determined by Hänel \& Reck by habitat type and priority.

Table 5.17: Mileage shares and vehicle factors III 
Table 5.18: Thresholds of daily traffic volume (dtv) of substantially increasing fragmenting impact.....

Table 5.19: Relating the fragmenting impact of dissimilar traffic volumes by factor weights.

Table 5.20: Factor weights and values of fragmenting impact calculated for a heavy duty vehicle $\left(\mathrm{v}_{\mathrm{t}<12}\right)$ driving a distance of $10 \mathrm{~km}$ for various traffic volumes.

Table 5.21: Impact of the route Hann.Münden, Hannover, Brunswick, to Hann.Münden [route 1]; distance of the trip: $354.5 \mathrm{~km}$; frequency of the trip: 40 trips per year.

Table 5.22: Impact of the route Hannover (Lower Saxony) to Wendelstein (Bavaria) [route 2]; distance of the trip: $479.9 \mathrm{~km}$; frequency of the trip: 73 trips per year.

Table 5.23: Comparison of the original and an alternative leg as part of an analysis targeted to mitigate spatial and fragmenting ecological impact.

Table Ap.1: Situation of payoffs which provokes an inefficient result. 180

Table Ap.2: Traffic volume impacts on wildlife at different thresholds. 


\section{List of Figures}

Figure 2.1: $\quad$ Main global direct drivers of biodiversity loss...................................... 8

Figure 3.1: European levels of landscape fragmentation by effective mesh size. ... 36

Figure 5.1: $\quad$ Standard cross-section 29.5 of a four lane highway as of RAS-Q and an indication of road elements................................................ 59

Figure 5.2: Examples of sound pressure levels in the human environment. ........... 75

Figure 5.3: Effects of noise on wild bird fauna: relative frequency of breeding birds in Dutch grasslands.

Figure 5.4: Distribution of the data sample generated from noise viewers of three German federal states.

Figure 5.5: Graphical representation of the correlation between traffic volume and noise zone extension from the obtained equations.

Figure 5.6: Graphical representation of the correlation between traffic volume and noise zone extension when adjusting the practical approach

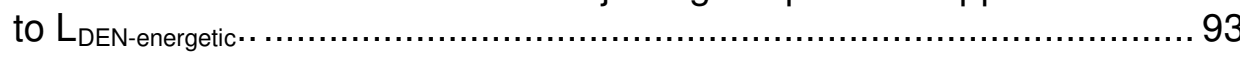

Figure 5.7: Noise levels and their extensions at peaking hourly traffic volumes......96

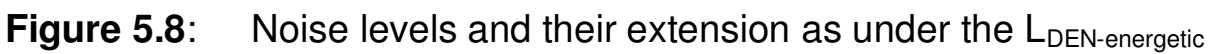
scenario for a German freeway at 64,000 vehicles per day.

Figure 5.9: Relation of sound pressure levels of passenger cars and heavy duty vehicles as calculated by various emission models used in Europe.

Figure 5.10: Cut-out of the map for large mammals for the region around Göttingen in Lower Saxony, 156 x 74 kilometers.

Figure 5.11: Image of an extending zone of conflict between Freeway 7 and the habitat corridor of forest living species north-east of Kassel, Germany.

Figure 5.12: Two locations of conflict forming one line conflict, but to be counted individually as they concern distinct corridors.

Figure 5.13: Display of the final part of route 2

Figure Ap.1: Map of the federal states of Germany. 190

Figure Ap.2: Route display Hann. Münden to Hannover to Brunswick and back to Hann. Münden.

Figure Ap.3: Route display Hannover to Wendelstein 



\section{List of Abbreviations}

23 CFR 772 Procedures for Abatement of Highway Traffic Noise and Construction Noise (USA)

ALKIS Land Survey Register Information System (Germany)

AR4 Fourth Assessment Report

B\&B Camp. Business \& Biodiversity Campaign

Bast Federal Highway Research Institute (Germany)

BfN Federal Agency for Nature Conservation (Germany)

BfU Bavarian Agency for Environment (Germany)

BImSchG 16 Federal Immission Control Act (Germany)

BKG Federal Agency for Cartography and Geodesy (Germany)

BMU Federal Ministry for the Environment, Nature Conservation and Nuclear Safety (Germany)

BMVBS Federal Ministry of Transport, Building and Urban Development (Germany)

BRD Federal Republic of Germany

CBD Convention on Biological Diversity

CML Institute of Environmental Sciences at Leiden University

CNCR Constant Nature Capital Rule

CNOSSOS Common Noise Assessment Methods

$\mathrm{CO}_{2} \quad$ Carbon Dioxide

COP Conference of the Parties

CORINE Coordination of Information on the Environment

COST 341 European Handbook for Identifying Conflicts and Designing Solutions

$\mathrm{dB}(\mathrm{A}) \quad$ Decibel (A-weighting)

DEGA German Accoustical Society

DFD German Remote Sensing Data Center

DIN German Institute for Standardization

DLR German Aerospace Center

DPSIR-model Driver, Pressure, State, Impact and Response Model

dtv daily traffic volume

EEA European Environment Agency

EEC European Economic Community

EIA Environmental Impact Assessment 
EKD Ecclesiastic Office of the Protestant Church (Germany)

EMAS Eco-Management and Audit Scheme

EU European Union

EU-ETS European Emission Trading Scheme

FGSV German Road and Transportation Research Association

FWIP Federal Wildlife Infrastructure Plan

G4-DMA Disclosure of the Management Approach of Guidelines 4

GDP

Gross Domestic Product

GFN

Global Footprint Network

GHG

Greenhouse Gases

GIS

Geographic Information System

GMO

Genetically Modified Organisms

GPS

Global Positioning Systems

GRI

Global Reporting Initiative

GVW

Gross Vehicle Weight

HDV

Heavy Duty Vehicle

IPBES

Intergovernmental Platform on Biodiversity and Ecosystem Services

IPCC

Intergovernmental Panel on Climate Change

ISO

International Standardizing Organization

IUCN

International Union for Conservation of Nature

IWW

Institute for Economic Policy and Research

LCA

Life Cycle Assessment

MA

Millennium Ecosystem Assessment

$m_{\text {eff }}$

Effective Mesh Size

MELS

Ministry of the Environment, Energy and Climate Protection of Lower Saxony

MKULV NRW Ministry for Climate Protection, Environment, Agriculture, Nature Conservation and Consumer Protection of the German State of North Rhine-Westphalia

MSV Applicable Hourly Traffic Volume (Germany)

NDLI Share of Noise Driven Landscape Impact

NeMoBFStr Network-model for Federal Roads (Germany)

NEPA National Environmental Policy Act

NMPB Noise Emission Model of Vehicles (France)

$\mathrm{NO}_{x} \quad$ Nitrogen oxides 
ODOT Ohio Department of Transportation

PCU Passenger Car Unit

ProgTrans AG Prognoses and Strategy Consulting for Transport and Traffic

RAA

Guidelines for the Design of Motorways (Germany)

RAL Guidelines for the Design of Country-roads (Germany)

RAS-Q Guidelines for the Design of Roads

REDD Reducing Emissions from Deforestation and forest Degradation

RLS-90 Guidelines of Noise Control of Roads (Germany)

$\mathrm{RQ} \quad$ Standard Cross-Section

RVS Guidelines and Rules of Roads (Austria)

SEA Strategic Environmental Assessment

SNR Signal-to-Noise Ratio

SonROad Modell of Quantification of Road Noise (Switzerland)

$\mathrm{SO}_{\mathrm{x}} \quad$ Sulphur oxides

SPL Sound Pressure Level

SSA Share of Sealed Area

TEEB The Economics of Ecosystems and Biodiversity

TNW Federal Highway Administration Noise Model (USA)

UBA German Environmental Agency

UN United Nations

UNA-Canada United Nations Association in Canada

UNESCO United Nations Educational, Scientific and Cultural Organization

UVPG Law on Environmental Impact Assessment (Germany)

$\mathrm{UZVR}_{100} \quad$ non-dissected low traffic areas larger $100 \mathrm{~km}^{2}$

VBUS Tentative Method of Quantification of Ambient Noise of Roads (Germany)

WHO World Health Organization 



\section{Variables Declaration}

\begin{tabular}{|c|c|}
\hline$A_{i=1, \ldots, n}$ & sizes of the individual areas or patches $\mathrm{i}$ with $\mathrm{i}=1,2, \ldots, \mathrm{n}$ \\
\hline$A_{\text {total }}$ & total size of the investigated region \\
\hline $\mathrm{cg}_{\mathrm{i}}$ & $\begin{array}{l}\text { conflict group } \mathrm{i} \text { indicating the severity of conflicts from high to low, } \mathrm{i}=1 \text {, } \\
2, \ldots 6 \text {. }\end{array}$ \\
\hline $\mathrm{D}$ & deviation from basic time-average sound pressure level $S P L_{m, 25}$ \\
\hline$D_{\text {at }}$ & atmospheric attenuation corrective \\
\hline $\mathrm{D}_{\mathrm{ba}}$ & barrier attenuation corrective \\
\hline$D_{\text {da }}$ & distance and air absorption corrective \\
\hline$D_{g m}$ & ground and meteorological attenuation corrective \\
\hline$D_{i}$ & inclination corrective \\
\hline$d_{i}$ & length of section $\mathrm{i}$ \\
\hline $\mathrm{d}_{\mathrm{iFH}}$ & distance travelled in section i on a federal highway in meters \\
\hline$d_{i F W}$ & distance travelled in section i on a freeway in meters \\
\hline$d_{i S R}$ & $\begin{array}{l}\text { distance travelled in section i on a lower category road ("smaller road") } \\
\text { in meters }\end{array}$ \\
\hline$D_{1}$ & line source \\
\hline$D_{\mathrm{ms}}$ & mirror sound sources corrective \\
\hline$D_{\text {rev }}$ & reverberation corrective \\
\hline$D_{\text {rs }}$ & road surface corrective \\
\hline$D_{s}$ & speed limit corrective \\
\hline$d t v_{i}$ & average daily traffic volume of section $\mathrm{i}$ \\
\hline$f\left(v_{x}\right)$ & vehicle factor of vehicle type $v_{x}$ \\
\hline $\mathrm{FV}_{\text {cha }}$ & $\begin{array}{l}\text { fragmenting value of a route using a corridor- and habitat-orientated } \\
\text { approach }\end{array}$ \\
\hline $\mathrm{FV}_{\text {dla }}$ & fragmenting value of a route using a discretionary landscape approach \\
\hline $\mathrm{h}$ & habitat type: wet $(w)$, dry $(\mathrm{d})$, forest $(\mathrm{f})$, forest living large mammals (Im) \\
\hline i & homogenous road section $\mathrm{i}$, with $\mathrm{i}=1,2, \ldots, \mathrm{n}$ \\
\hline $\mathrm{L}_{\text {car }}$ & time-average sound pressure level per passenger car per hour \\
\hline$L_{D E N}$ & time-average sound pressure level for day, evening and night \\
\hline$L_{D E N-e n e r g e t i c}$ & purely energetic time-average sound pressure level \\
\hline $\mathrm{l}_{\mathrm{i}}$ & number of lanes in section $\mathrm{i}$ \\
\hline$L_{\text {Night }}$ & time-average sound pressure level for nights \\
\hline$L_{\text {truck }}$ & time-average sound pressure level per heavy duty vehicle per hour \\
\hline $\mathrm{m}$ & meter (unit of measure) \\
\hline
\end{tabular}




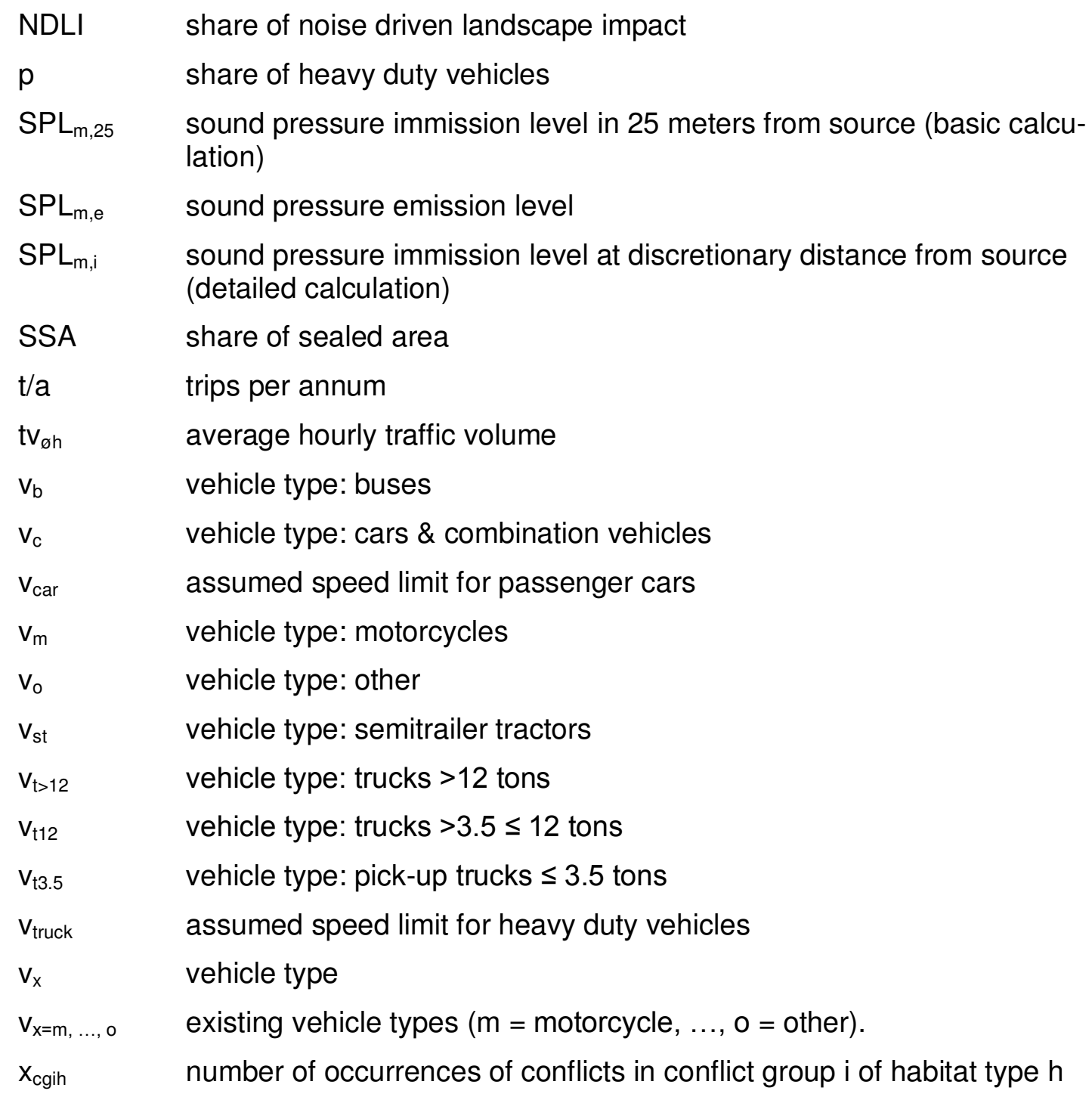






\section{Introduction}

Land use and fragmentation are among the major causes of the destruction of habitats and the resulting loss of biological diversity throughout the world (Chapin III et al. 2000, Sala et al. 2000). Climate change, affecting many more species more severely than our own, is expected to become a progressively more significant threat in the coming decades (CBD 2010a). Societal and political pressure, existing technological potential of improvement, and practicable measurement, in the first decade of the $21^{\text {st }}$ century made the emission of greenhouse gases the predominant ecological impact of concern in many countries and also in corporate environmental management. Awareness of the second environmental meta-crisis effected by humankind, namely the loss of biological diversity, seems to benefit from the recognition of climate change, because changes in climate concern many species and also because the possibility to ponder how long, under which conditions, and with which species around humans want to live anywhere on the earth, and what the respective implications on our actions would be, was activated anew by the characteristics of both crises.

Despite a range of environmental impacts of products and processes has been considered in life cycle assessment, having formed software programs that allow for identification and better management of individual effects, also in business the focus has been on pollutants and $\mathrm{CO}_{2}$-equivalent emissions. This focus is particularly true for the transport sector. The term "green logistics" in the past referred to greenhouse gas improved haulage processes that inter alia were obtained by emission reductions from route optimization, mode choice, the usage of tires of low rolling resistance, driver training, or adjusted warehouse management (Dekker et al. 2012, Murphy \& Poist 2000). However, companies have to improve further their sustainability reporting because the continuous development of environmental management tools and consumers demand for it, as overall problem awareness and the need for abatement increase. Likewise, the employment of the word sustainability may become more difficult, as sentience that the concept requires a comprehensive rather than a scattered approach toward dealing with adverse environmental effects also augments. In advancing the quantification of selected, underrepresented impacts, the goal of this research is to contribute to more complete corporate environmental management. To this end, after referring to the broader research context in Chapter 2, in Chapter 3 the available methodology to report and to manage environmental impacts of road haulage is analyzed. The finding that quantifiable indicators are missing for impacts other than direct land use and substance emissions, leads to selected further impacts being investigated and described in Chapter 4. As a result, degradation of area adjacent to roads and fragmentation are identified as significant impacts that need also be considered and reduced. As a precise assessment of impact makes it easier to manage a matter, methods that allow a 
quantification of the impacts identified in Chapter 4 for the unit of an individual road user are developed in Chapter 5.

Besides methodologies that concern fragmentation and degradation of adjacent area, an indicator measuring the individual share of sealed area is devised. This is because the impact also is found to be undervalued, because it is closely related to the impact of degraded adjacent area, and because the accuracy of the currently employed method of measurement is regarded to be imperfect. Also in Chapter 5, the implementation of the newly proposed indicators is tested for two exemplary transports and remaining limitations are named. The results mirror some of the characteristics of the formulae and confirm their functionality as the most significant numeric improvements coincide with the largest expected ecological amelioration. In Chapter 6 it becomes clear how, the presentation of philosophical analyses, concepts, and implications of humans' ends and values with regard to non-human nature, and the depiction of structural constraints to sustainable conduct in business and economics of Chapter 2, first and foremost the material growth paradigm and the at some point unsustainable throughput, relate to the development of environmental indicators. Regarding the impacts of land use and fragmentation, for firms and infrastructure planners both mitigation and avoidance display possible strategies of abatement. Yet, the potential of the latter is distinctly greater. At the total national level, mitigation alone is estimated to be insufficient to reach the political targets of reducing impairments caused by traffic by the means of pollutants, noise, and light, and of reaching conditions where existing transport routes no longer cause significant impairments to the system of interlinked biotopes or to the ecological passability between dissected areas (BMU 2011). This is not to say that road haulage clearly must be avoided, only it is to show what the current conditions of impacts are and that there is a need to consider both our ethical understanding of our relations to contemporaries, future generations, and other life forms, and our individual and collective behavior in order to act more consistently and to reach more satisfying results. In a summary in Chapter 7, the lines of action and findings of each chapter are briefly recited.

This work consists of the present text and four articles, which made the following contributions:

- Article 1 is entitled "Potential impacts of road haulage on biodiversity". It briefly identifies shortcomings of corporate environmental management concerning road haulage, and, short of the impacts of erosion, sedimentation, and changes in hydrology, the text in land degradation, fragmentation, road mortality, substance emissions, invasive alien species, and life-cycle impacts describes the principal ecological impacts of roads and vehicles. Interim methods of measurement of selected impacts are introduced. 
- Article 2 is entitled "Current practices of mitigating the ecologically harmful effects of roads - An assessment". It discusses recent conditions and trends of the effects of land use, material emissions, noise and light, and fragmentation in Germany. The focus is on the presentation and debate of the two major strategies of defragmentation that are currently applied. They can be referred to as a corridor-centered and an area-centered strategy. Advantages and weaknesses of both strategies are laid out in order to support finding a favorable weighting of the two strategies, as in fact both strategies need to be applied. Regarding the further investigated impairments, the positive trends of reductions are too weak to reach established targets, meaning that further improvements as well as improved monitoring are necessary.

- Article 3 is entitled "Traffic induced landscape fragmentation in the Biobío Region of Chile: Analysis and prospects". The text presents results of an analysis of landscape-level fragmentation in the Biobío Region. The study originates from the assumption of the Biobío Region not yet to be highly fragmented by transport infrastructure, and from the understanding that the most effective and most feasible approach to tackle fragmentation is to be aware of it before it establishes, in order to attempt avoiding it from the beginning. Results reveal that the overall level of fragmentation in the Biobío Region is moderate, meaning that significantly negative impacts are limited to specific species and locations. Strategies of mitigation and avoidance, such as bundling traffic or supporting sustainable resource use, are recommended for already severely dissected locations of ecological importance.

- Article 4 introduces the four methods of quantification that are explained in great detail in Chapter 5 of this text. The applied methodologies are explained and the case study of this text is used to illustrate the mode of operation of the indicators. Moreover, potentials and limitations of the formulae are specified. The indicators are intended to allow businesses to measure the respective impacts caused by their individual road transport activity. Results can be used for reporting in the context of LCA and for management and mitigation.

Findings of the research of the first three articles contributed to the eventual formation of methods of quantification of selected impacts introduced in Article 4. The following text focuses on explaining the need (Chapters 2, 3 and 4) and the courses of action pursued to develop the four final indicators. Potentials and limitations inherent to the formulae and regarding their contribution to mitigation are indicated (Chapters 5 and 6). 



\section{Fundament}

While the more immediate reasons that triggered the emergence of the research goal are explained in Chapters 3 and 4, in this chapter three topics that are closely related to environmental impact are introduced. Firstly, scientific results on biological and physical changes caused by human activity that are underway on planet earth are referred to. Their undesirability is the superordinate reason to consider and to reduce environmental impact. The questions, why certain impacts are undesirable, a question which $21^{\text {st }}$ century societies fail to answer and are reluctant to be aware of as for instance in the case of setting and reaching biodiversity goals, what the relation and potential responsibility for future generations and non-human life forms is like, and continued raveling on the self-identity of the human being and respective implications, in brief are turned to in the second part of this chapter because they are regarded important for humans' quests to live flourishing lives and for the chances to achieve a meaningful degree of sustainable behavior. This also concerns corporate decision-makers and people working in environmental management because their consideration of impacts and goal-setting require orientation, which shall be a conscious responsibility that cannot fully be ceded to regulation or consumers. Moreover, considering the wider meaning of effects allows making better informed decisions. This may be more fulfilling and may increase one's internal motivation for one's efforts. In the third subchapter, the use of the term sustainability and the condition of sustainable behavior in German society are reflected upon. In addition, five constraints faced by the realization of sustainability in the economic and corporate field are identified. Despite these constraints occur at the level of the overall economic system, they have significant implications on the environmental management efforts of individual corporate entities. As not only the theoretical formulation of indicators, but also their application and the management of the impacts are objectives of this research, in order realistically to estimate their potential, it is viewed relevant to consider the broader context of sustainability in economics and in business. However, apart from a description of an economic and societal model aiming to reduce gross material throughput, which later will become relevant in the analysis of the abatement of impacts, a detailed analysis of the constraints is not central to the research question. Therefore, the analysis is placed in Appendix B of this work.

\subsection{Groundwork of the Natural Sciences}

Since the enlightenment in the late $17^{\text {th }}$ - and $18^{\text {th }}$-century, natural sciences have a powerful twofold significance for Western societies. While the more obvious value lies in the at first theoretical, and subsequently applied dimension of a better understanding of natural processes, the enlarged knowledge of the character of particular life forms 
and causes and effects has a philosophical implication because it invites to an anew assessment of human relation to these life forms, the result of which may concern individual actions and collective organization. Extended knowledge on other life forms must not necessarily go into their valuation by humans, but should provoke a situation of conflict and eventually lead to change, if it stands in contrast to established beliefs (cp. Eichhorn 2013). While the dimension of enlarged knowledge is valid from the moment of discovery, the dimension of comparing and adjusting belief systems to then trigger according behavior endures much longer. This can be seen in two significant crises of the present. While the causes of climate change and loss of biodiversity are known and the consequences of business-as-usual scenarios are theoretically objected to, a development of values concerning the relation to future generations or to nonhuman species is still largely inexistent across society and far from having advanced enough to guide one's actions and to distinguish between right and wrong (Becker 2010). If people in Western societies, as busy and in many ways disconnected as they are at the beginning of the $21^{\text {st }}$ century, are at all interested and only then capable to confront such task, is uncertain. In the following sections 2.1.1 and 2.1.2, an overview on the history and the findings regarding the loss of biological diversity and on the continually emerging research field called 'road ecology' are presented.

\subsubsection{The Condition of Biological Diversity}

Biodiversity is the variety of life in all its forms. It is short for biological diversity and represents the variability and totality of species of flora and fauna, of genes within species, and of ecosystems. It is valuable to humans on the one hand because some people think that it has a value by itself, and on the other hand because humans obtain benefits from it, which range from life sustaining functions of ecosystems, such as soil fertility, oxygen production, $\mathrm{CO}_{2}$-absorption, pollination, clean water, etc., to the quality of life augmenting, immaterial experiences and sensations around nature, such as beauty, reverberation of life, relational perceptions from friendship over fear to responsibility, spiritual and cultural aspects, etc. Although the meaning of the term biodiversity to a certain degree resembles other ecologically denoted terms like nature, ecology, or environment, the word biodiversity underlines the fact that there is a rich diversity of living organisms that live in concurrence in equally diverse ecosystems.

With regard to the recent history relevant for the present valuation of losing biodiversity, several findings and institutions played central roles. A first milestone in identifying and communicating overall non-sustainable human behavior in Western countries was the foundation of the Club of Rome 1968 and its publication 'The limits to Growth' by Meadows et al. in 1972. The elementary principle is the members' common concern for the future of humanity and the planet. This translates into the working focus of detect- 
ing large scale problems and proposing possible solutions (Club of Rome 2011). Twenty years later, the Convention on Biological Diversity (CBD) emerged from the United Nations Conference on Environment and Development, also known as the Earth Summit of Rio de Janeiro in 1992. The foundation of the CBD originates from the recognition that the Earth's biological resources are vital to humanity's economic and social development and that they are a global asset of tremendous value to present as well as to future generations (CBD 2011). In 1992 the CBD was founded and members among other key statements affirmed, that the conservation of biological diversity was a common concern of humankind (CBD 1992).

Although established already in 1988, the Intergovernmental Panel on Climate Change (IPCC) had its first major impact in politics and the media with the publication of its Fourth Assessment Report (AR4) in 2007 (IPCC 2007). On the basis of this report, the internationally agreed upon limit of global warming of two degree Celsius was settled as the Copenhagen Accord in December 2009 in the United Nations 15th conference of the parties (COP 15) (UN \& UNFCCC 2010). Although the first comprehensive publication concerning the loss of biodiversity and the conditions of the earth's main ecosystems was published as the Millennium Ecosystem Assessment (MA) in 2005, and therewith two years prior to the AR4, the main impact of the study came to pass only after the topic of climate change had been made popular by the Fourth Assessment Report of the of the IPCC and by the attention that was generated before and after the COP 15. The MA confirmed the results of two recognized study by Sala et al. and Chapin III et al. in 2000.

Results of the Millennium Assessment continue to function as a basis of current scientific research and political decision- and policy-making. The findings were not outdated, but rather reaffirmed by the latest comprehensive publication of the CBD in 2010 on the conditions and trends of global biodiversity (CBD 2010b). The Millennium Assessment had two main objectives: firstly, to assess the consequences of ecosystem change for human well-being, and secondly, to establish a scientific basis for actions needed to enhance the conservation and sustainable use of those systems (MA 2005). Results of the MA revealed that over the last 50 years ecosystems have changed at a pace unprecedented in human history. Gains in human well-being and economic development had been achieved at the cost of degraded ecosystems, which were likely to become incapable of producing the existential, economically favorable and the quality of life enriching benefits to humans in the future, should they be degraded much further (MA 2005). Figure 2.1 shows that land use, climate change, overexploitation, invasive alien species, and pollution were identified as the five main direct threads to biological diversity. Land use still is the dominant negative factor; climate change is on a worrisome 
rise and expected to become the most severe problem in the future; and the negative effects from invasive alien species also are estimated to increase (MA 2005).

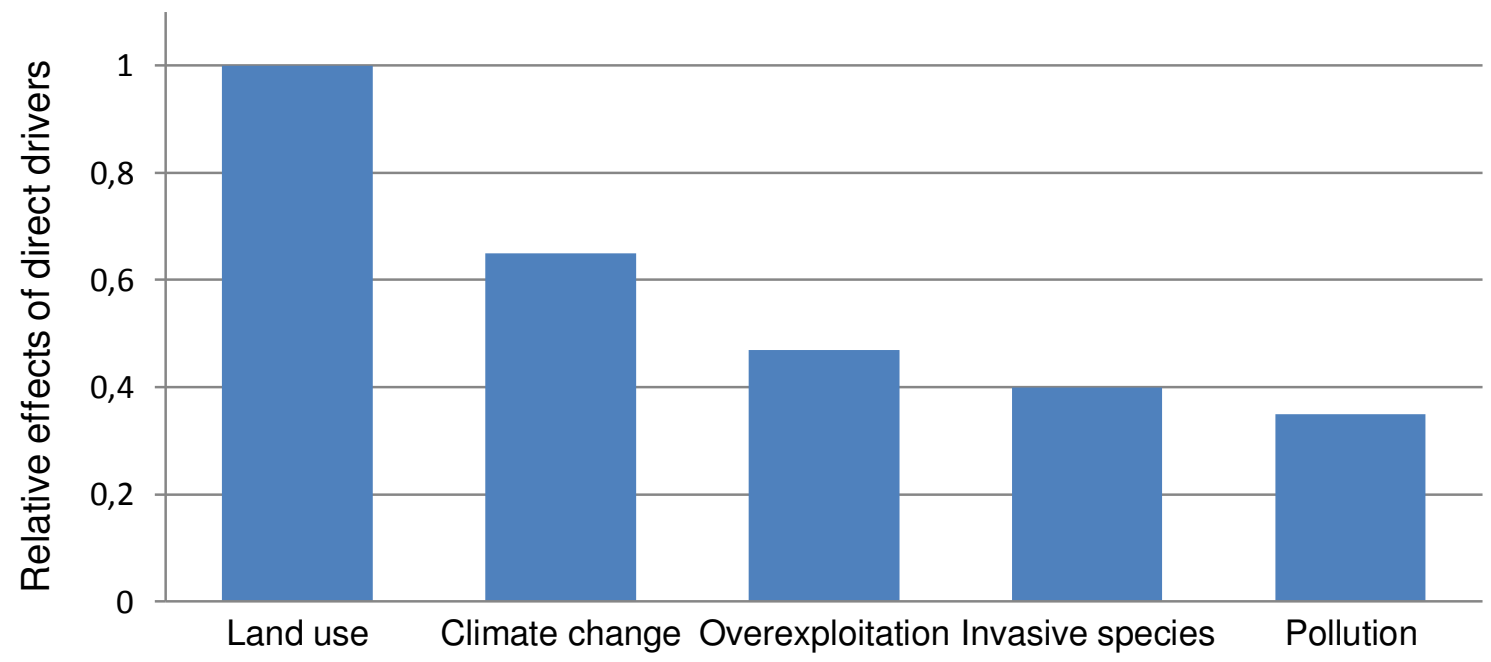

Figure 2.1: Main global direct drivers of biodiversity loss (Source: adapted from Sala et al. 2000, MA 2005).

Results and studies of the above mentioned organizations became important sources of information in politics, the media and for researchers. In December 2013, 178 or $92 \%$ of all parties of the CBD had developed national biodiversity strategies and action plans (CBD 2013). The United Nations concluded to end the 'Year of Biodiversity 2010' with the announcement of the beginning of an entire 'Decade of Biodiversity 2011 to 2020'. Beginning in 1960, the list of International Decades Designated by the General Assembly in 2011 consists of more than 30 decades, concerning topics like industrial development for Africa, drug abuse, combat against racism and racial discrimination, or deserts and the fight against desertification (UN 2011, UNA-Canada 2011). The 'Decade on Biodiversity' is decade no. 37. Although an announcement alone does not solve an issue, nor does it necessarily establish the issue as a priority throughout society, the formulation of national biodiversity strategies and the increasing implementation of impacts on biodiversity in environmental management suggest a rising relevance of topics related to biodiversity across the global society (cp. GRI 2006 \& 2011). Moreover, in 2011, the 'Intergovernmental Platform on Biodiversity and Ecosystem Services' (IPBES), an international, scientifically independent organization that focuses on ecosystem development was founded. The objectives of the institute are to follow up on the findings of the MA and to strengthen the scientific basis of global biodiversity and ecosystem services research. The institute is meant to serve as an interface between the scientific community and policy makers in order to reinforce the consideration of science in policy making (IPBES 2011). 


\subsubsection{The Research Field 'Road Ecology'}

When considering the ecological impacts of transport infrastructure, the effects of land use and fragmentation are not the only ones. Yet, these two were selected to be represented by indicators in the scope of this work. The ecological reason why they were chosen is their severity of impact. They are estimated in most cases to be the two largest threats to biodiversity and ecosystems (Oggier et al. 2007, Andel et al. 2010). The development of the indicators draws upon a discipline of the natural sciences that is called 'road ecology'. It was brought into being by a publication from Stoner in 1925, advanced significantly in the 1970s and 1980s by publications from Mader and Ellenberg, and had its international breakthrough in 2003, with the publication of Forman et al.'s book entitled 'Road Ecology' (Stoner 1925, Mader 1979, Forman et al. 2003). The research field appears still to be growing and it has become essential in accompanying infrastructure planning (Roedenbeck \& Jaeger 2006). An example is the European research program COST 341 , which was set up in order to share knowledge on fragmentation between European countries and in order to reduce fragmentation, with particular regard to to be built transport infrastructure in Eastern Europe (Oggier et al. 2007). Road ecology is defined as the interaction between organisms and their environment, linked to roads and vehicles (Forman et al. 2003). The main goal in road ecology research is systematically to record and assess the ecological impacts of roads, in order to lay a foundation for avoiding and mitigating significant negative impacts in the future (van der Ree et al. 2011). Reviewing the literature, for the last five to ten years the impact of habitat fragmentation seems to have been at the center of research and discussions. Current efforts of research predominantly tackle the subjects genetic consequences of fragmentation, functionality of crossing structures to abate population-level effects, and, relatedly, analysis and complementing of strategies apt to restore differing qualities of connectivity (Friedrich \& Geldermann 2013b).

\subsection{Philosophical Context}

The need to ponder their relation to, their valuation of, and their responsibility for nonhuman nature, for future generations, and for contemporary minorities who value nature to a higher degree than themselves, applies to most people in most societies around the world. However, in order to set goals, to devise strategies, and to facilitate the behavior necessary to reach the goals, for the complexity of relations of cause and effect, politicians, decision-makers, and experts have particular responsibility to consider the above questions. That efforts and goals in the realm of sustainability are much debated and often inconsistent, is evidence of the immature state of societal discussion of sustainability-matters. Reading and reflecting on the concepts and thoughts 
presented on the following pages will not close, but potentially contribute to narrow this gap. Thereto, a brief introduction to ethics, a description of the currently most prominent delineation of humans' valuation of all of nature, and the presentation of Nussbaum's and Becker's concepts, findings and thoughts of which in specifying concrete implications transfer the theoretical topic to personal and societal realities, are given. Regarded to be relevant to all people and to those who are more knowledgeable and who carry public responsibility in particular, to provide and allow for a certain degree of ethical reflection is considered an indispensable part of a work that deals with ecological impact. A simple way to embed the philosophical consideration of the research goal of quantifying ecological impacts of road haulage is to ask the question: why is the goal set? This implies the query, what the achievement of the goal shall lead to.

\subsubsection{On the Relation of Humans and Non-Human Nature}

The goal may have been set because humans are provoking an environmental crisis. Building on that, and as the word crisis suggests, there is a consensus that the biotic and abiotic changes underway are undesirable, at least to some extent. This assessment shows in the ratification of the Convention on Biological Diversity, in the resolution of the Copenhagen Accord, which set a target for one of the characteristics of climate change, namely for average global temperature not to increase beyond two per cent, or in individual peoples' efforts attempting to mitigate and to limit "harmful" environmental impact of their lives. Reuter (2013) correctly reasons that the changes taking place are best described as an environmental crisis and not as ecological crises, because the crises are not innate to ecosystems, species, or the climate, but are related to humans who for similar cultural aspirations are both shaping the changes and are affected by the changes in what they regard their environment. The finding that humans are producing the changes and that they are affected by them is no sufficient answer to the question raised above, but it leads the right way.

While 'shaping' and 'being affected' are rather vague expressions, their meaning can be concentrated to the central question in ethics, namely how one should live, or which ultimate ends and values one should orient one's actions towards. Krebs (1997) distinguishes between two groups of ends or values: eudemonic values, such as selfactualization in the job, personal health, or travels to foreign countries, which are related to one's own good and flourishing life, and moral values like gender equality, a fair sharing of resources, or the well-being of people living in foreign countries, which imply that one also gears one's actions to the happiness and interests of others. With regard to the human relation to non-human nature, 'shaping' and 'being affected' more precisely refer to the questions, whether non-human nature can contribute to a happy and flourishing life and thus be of eudemonic value, and whether one should respect the 
ecological interests of contemporary and future human beings and potentially of all biotic and abiotic nature, recognizing a certain moral value of nature.

In this paragraph a brief aside on the relatedness of eudemonic value and moral value is stated. While the overall distinction between the two different motivations for one's actions appears appropriate, one can argue that, probably as most species, humankind would not have remained to exist, if building and adhering to moral ends and values that target the well-being of others besides subconscious thoughts of benefits in security and sexuality, would not also be eudemonic itself. It seems that while eudemonia, which can be described as human flourishing, is not limited to happiness but includes the central experiences of human life like conviviality, sleep or study, having and adhering to morale cannot be said to be free of producing eudemonia and happiness. This condition Krebs falls short to refer to. Let's assume the case of Kedi. When leaving a room, Kedi does not turn off the light in order to feel happier, but because her sense of justice of the conflicting demands of all other living things suggests her to. In thinking of them at that moment and in feeling closer to and being less afraid of them when meeting some of them later on, she strengthens and 'improves' (i.e. less fear, more community) her relation with them and this makes her happier. The importance of relations is underlined by Becker, who views the human being to be "a fundamentally dependent and relational being" (2010, p. 3). While it is possible or even natural also to have unpleasant feelings when thinking of others when turning off a light, for instance because some people do not share one's sense of justice which makes oneself feel abused, it is presumed that in following one's moral values, the feelings of happiness usually outweigh the feelings of discomfort. While the conscious reason to build and adhere to moral values is acknowledged not primarily to be self-centered and driven by eudemonic experience, categorizing moral value as distinct from eudemonic value with regard to eudemonia is viewed implausible. While Hume's conception of the divergence of the two fundamental moral human sentiments, which to him are egocentric self-love and altruistic sympathy (Wolters 1995), supports the distinction of self-centered and unselfish motivations for actions, regarding eudemonia both sentiments are likely to produce good feelings and it is difficult to say, if one outweighs the other. Specifying the eudemonic potential of moral values was regarded an important annotation when distinguishing between the two groups of values to orient one's actions towards.

If eudemonia originating from self-love, which can include relishing nature's beauty, and solidarity, and moral values of mutual consideration allow drawing near the goal of human life, which Aristotle thought to be 'the good life', which for him is eudemonia in its ultimate form, then humankind should attempt to become aware of the meaning of non-human nature in this context. Despite the urgency, Ott (2009a) prospects hope as he opines in the style of Kant that while the majority of $21^{\text {st }}$ century societies do not live 
sustainably, they have begun to think of the value of nature. 1 To this end, in continuation the classical approaches of classifying human valuation of nature are presented. Thereafter, the two selected, promising concepts of Nussbaum's 'Capabilities Approach' and Becker's 'Sustainability Ethics', which are regarded to be able to contribute to answer the question on how we should live, and which in the case of approving of them have direct societal implications, are turned to.

When analyzing valuations of nature, the major distinction between different descriptions lies in the assumption either to recognize an innate moral value in parts or in all of nature, which is called physiocentrism (from Greek "physis" nature), or not to grant forms of nature an intrinsic value, a position called anthropocentrism (form Greek "anthropos" = the human being). Only at a rarely plead extreme of the latter position is it considered morally righteous for humans to dispose of nature to their free liking. When granting intrinsic moral value to parts of nature, e.g. to animals, it is possible that the character of this moral value is different from the moral value for instance granted to humans. While this is referred to as hierarchical physiocentrism, accepting equal moral value is considered egalitarian physiocentrism (Krebs 1997). Assuming innate moral value of different parts of nature has formed three major variants of physiocentrism: pathocentrism recognizes moral value in all sentient beings that can feel pain and suffering, biocentrism extends the appreciation of value to all biotic life forms, potentially due to Rolston's argument that organisms, if not moral, are at least axiological systems because they differentiate between an actual and a nominal condition and strive for the nominal condition (Rolston 1994) or due to the recognition of all living beings' autotelicy, which is the acceptance of them to contain their own meaning and purpose and to have an innate desire to survive and unfold, and radical physiocentrism, deep ecology or ecocentrism sees moral value in all of nature as for instance defined in footnote 1.

When moving from the reductionist extremes of regarding nature to be of purely instrumental value or of absolute value to the moderated perspectives of either a nontruncated anthropocentrism, which allows to see in nature a variety of values, such as aesthetic, spiritual, or relational values, that can produce eudemonia in humans and that humans can enjoy without needing to exploit nature, merely benefitting from its presence and a certain treatment of respect, or an epistemic-anthropocentric physiocentrism, which acknowledges the active and subjective role of the human being in assessing and recognizing intrinsic moral value in other species and which passes

1 Nature in the following refers to all biotic life forms, including humankind, to all abiotic objects, such as water or soil, as well as to geophysical systems like the climate system or moon cycle. 
some elements of human culture like care or enjoyment to parts of nature, most human attitudes and sentiments for nature can be described and understood (Krebs 1997). Valuing nature from either perspective is likely to contribute to a flourishing life, because either appreciating nature for its resources like soil fertility and food, shelter, air, or rain, for its beauty, reverberation of life, the felt relational aspects like friendship, respect, or care, for its relevance for the meaning of life, or for nature to produce a sense of home, which technically all stem from an anthropocentrically dominated perspective (Krebs 2008), or when having a physiocentric motivation to value nature, partially including it as a character of dignity that has innate moral value and that humans then include to orient their actions towards, which is likely to create a larger and more related community and to increase the number of potential recipients of sympathy and solidarity, are likely to be effective in this regard. Importantly, it is possible for one person to appreciate nature for sentiments from both perspectives. This concludes the overview of the established delineation of dissimilar types of valuations of nature. In the following, two promising concepts that are regarded to be of avail to answer the question how we should life, a question that directly concerns ecological impact and environmental management, are presented. The first concept well exploits the theoretical valuation of nature referred to above and the second investigates and relates the character of the human being to the challenge of sustainability under dominating current societal conditions.

\subsubsection{Insights from Nussbaum's Capabilities Approach}

Nussbaum's capabilities approach affirmatively answers the questions of instrumental, including relational value, and moral value of nature in two ways: firstly, because she regards the relatedness to nature as a characteristic of the human being, which required for humans "to be able to live with concern for and in relation to animals, plants, and the world of nature" (Nussbaum 2006, p. 77), and secondly, she set the dignity of all beings as the premiss of her approach, dignity in this case meaning to demand treating all beings with the attention and respect to which they were entitled, hence suggesting certain rights to be held also by non-human beings.

Taking a step back, the goal of Nussbaum's concept is the philosophical reasoning of fundamental constitutional principles of societies that shall be accepted and adopted as the absolute minimum of what the respect of human dignity requires (Nussbaum 2000, p. 5). Inspired by the Doctrine of Virtue of Aristotle and Marx, Nussbaum builds on and surpasses the works on the conception and dignity of the person of Kant and Rawls as she expands the apprehension of dignity from the rational to an emotional and sociable dimension (Nussbaum 2006). While critics of metaphysic essentialism argue that nothing about the character of the human species and the world can be known for certain, 
Nussbaum believes in the epistemic quality of internal essentialism, as she views that a deep examination of human history and human cognition through the cognitive apparatus of the human being itself can reveal a fairly determinate account of certain characteristics of human beings (Nussbaum 1992). Following such analysis, she regards it to be possible to become aware of universally valid, fundamental conditions that allow for a flourishing life. She names these conditions 'capabilities' and reasons each dignified human being shall be entitled to them in order not to be restricted in leading a flourishing life. While she sees her list of ten capabilities as open and changeable, she is positive that it can be valid independent of cultures and religions and she hopes that it can be an overlapping consensus between people who otherwise hold comprehensively different views (Nussbaum 2006).

Like the first capabilities approach developed by Sen, which centers on functions of freedom that allow a person to live self-determined (Sen 1999), also Nussbaum's concept was originally designed as an alternative to narrow economic-utilitarian indicators of individual and societal welfare being used at that time, such as gross domestic product (GDP). More than a decade later, GDP still is the most common index to be calculated and communicated. However, awareness of the shortcomings of the use of GDP as an index of well-being and welfare, such as, inter alia, the inabilities to assess happiness, health, regional economic independence, guarantee of human rights, etc., to consider the balance of ecosystem and natural resource availability, and even to account for the distribution of monetary wealth, have reduced its credibility to a low level. Inspired by Aristotle, who urged to be clear on what the equality and inequality of people should consist of (Aristotle 1282), and the philosophy of young Marx, who foresaw a new political economy in which not wealth and poverty, but the rich human being as the human being in need of the totality of human life activities, "the man in whom his own realization exists as an inner necessity, as need", would be the reality, (Marx 1844/1978), Nussbaum chose to use the conception of dignity in order to press for social and political institutions to monitor and to install minimum thresholds of the unalienable capabilities necessary for a good life. Being able to live in concern for and in relation to animal, plants, and the world of nature is one of the ten capabilities identified by Nussbaum. Her seeing her concept close to the one of human rights, well classifies her evaluation of the importance of human relation to nature (Nussbaum 2006). Nussbaum's assessment can be seen as a partial answer to the initial question why to develop indicators that allow for the assessment of environmental impact. In the next paragraphs, a résumé of the analysis of the ethical dimension of sustainability by Becker is presented. Becker's analysis also is viewed to allow for additional insight on the valuation and relation of the human being to non-human nature. 


\subsubsection{Implications of Becker's Analysis of the Sustainable Person}

Becker's central hypothesis is that we would struggle with the concept of sustainability, or the question of how we should live in relation to non-human nature, because the concept was no lightweight test, but concerned the fundamental philosophical issue of the self-identity and the existence of the human being as a dependent and relational being. 'Sustainability' for the first time would force the human being to acknowledge and to deal with a relation not only among contemporaries anywhere in the world, but also with nature and with past and future generations. While Becker's view of the human being to be dependent and relational to a significant extent was largely inspired by the approaches of virtue ethics and ethics of care, which belong to the approach in normative ethics that emphasizes virtues, moral character, and relations and stands in contrast to the approaches of deontology, which emphasizes duties or rules (e.g. Kantian ethics) and consequentialism, which emphasizes the consequences of actions (e.g. utilitarianism)2, also theories in the natural sciences support the significance of the relational character of the human being. For instance does the sociobiological finding, that altruism is likely to be evolutionary beneficial support the biophilia hypothesis, saying that the sentiment of altruism, which originally was rooted in the child-parent relationship, over the course of human cultural development expanded to larger entities, i.e. tribes, the human species, and eventually all of nature (Dawkins 1976, Wolters 1995). Partially for humans' genetic relatedness to other organisms, Wilson in his variation of the biophilia hypothesis emphasizes the foundation of biophilia to lie in humans' innate affinity for nature that made it a necessity to be in sufficient contact with nature in order to remain healthy, to see life's meaning, and to self-actualize (Wilson 1984).

For Becker sustainability also contains the characteristics of continuance of certain 'things' or systems and of positive normative orientation, as for instance in 'sustainable development', but it essentially is about the constitution and development of fundamental relationships of the human being, which can only thrive at the individual level, if the systemic level allows and facilitates so. At the individual level of morality, the three fundamental relationships of the human being can be respected by developing a type of

2 Suppose it is obvious that someone in need should be helped. A utilitarian will point to the fact that the consequences of doing so will maximize well-being, a deontologist to the fact that, in doing so the agent will be acting in accordance with a moral rule such as 'Do unto others as you would be done by' and a virtue ethicist would reflect what helping or not helping would say about her or his character or moral behavior, with the result to then help because it is benevolent and in accordance with her or his moral values (Hursthouse 2013). Still she or he would decide on a case-by-case basis, which reflects the negation of the absolute 'justice view' of morality by ethicists of care for whom morality rests on the understanding of relationships as a response to another in their terms. 
self-identity of a sustainable person. Such a person's characteristics would be: first, a relational self-identity as a temporal, interdependent, culturally and naturally contingent being, second, relational virtues or competences of attentiveness and receptiveness, third, further relational values of sympathy, such as respect, care, and responsibility, and fourth, an encompassing understanding of the human being as an emotional, rational, communicative, and creative being (Becker 2010, p. 7). The challenge of personal development would be the experience and adoption of these characteristics and their integration into the three relationships. Can this be achieved, ideally, all parts could flourish.

For the sustainable person to flourish, Becker regards the systemic level to be important because relationships to a large extent would be mediated by social structures, patterns of thought, education, etc. That Becker finds them today to significantly hamper the development of the sustainable person, he exemplifies by analyzing three fundamental global meta-structures, science, technology, and economy, to all of which the following three findings would apply: first, a definition of the human being as exclusively rational, putting humans in an active role and making nature a passive object of humans' cognition, control, and use. In addition, persons would increasingly understand themselves as rational utility maximizers and also see others as economic persons. This affected the relationships between contemporaries at the local and global scale and lead to an ethically problematic reduction of the human being and its relations. Second, the two dominant ends of autonomy of the individual and of growth-paradigms of more being better than less would stand in conflict with the sustainable person and its self-identity. Third, increasing complexity in all three meta-structures would complicate it to relate elements, which would impede the potential of individual responsibility. Concluding, Becker formulates the needs of a new, encompassing concept of the human being and of human rationality, of a basic reevaluation of most meta-structures, particularly science, and of a stability and simplicity of meta-structures. While Becker sees one task of philosophy to be the critical consideration of the underlying dynamics of current societal and global issues like sustainability, biodiversity loss, or economic crises, Eichhorn (2013) opines that moral philosophy in addition should consider the findings of the natural sciences on the character and the development of the human species and on other species more thoroughly than that had been the case for the last 200 years. Crompton and Kasser (2009) agree with Becker on the terms that in order to successfully confront the profound environmental challenges facing humanity, they recommend to become aware of and to use the environmentally-helpful aspects of human identity to a higher degree.

While Becker does not directly refer to eudemonia and while he 'only' answers how the sustainable person would live, it seems that, besides the alternative to live sustainably 
being unpleasant and of limited temporal success, the sustainable person in her selfidentity and in the quality of her relations, compared to the utility-rational and autonomous one, is the one of higher potential to self-actualize and to sense eudemonia. Aristotle assents to the importance of relations as for him the virtues function as means to safeguard human relations (maybe including those to nature), and here particularly authentic friendship, without which one's quest for happiness would be frustrated. Rosa (2014) concludes that missing 'experiences of resonance' or alienation in various dimensions like consumption, politics, work, or oneself, played a major role in stagnating levels of happiness and the increase in nervous system diseases of people of busy, competitive Western societies.

All four subjects, the two-dimensional approach of eudemonic and moral values to ethics, the delineation of dissimilar types of valuation of nature, Nussbaum's concept of relating the connection to nature to human dignity and thus to the flourishing life, and Becker's analysis of the ethical dimension of sustainability, which makes sustainability not just a concept to apply but an earnest demand to reflect on the character of the human being and on current societal conditions to support or to obstruct its evolvement, were considered adjuvant sources of inspiration in order to ponder how we should live with regard to our relation to nature and in order to ponder and to gain some idea of the ultimate goals of environmental management.

\subsection{Conditions and Constraints of Sustainability in Society}

After having referred to ecological conditions and selected philosophical concepts, in this chapter the term sustainability and sustainable conduct in society, as well as five structural economic constraints that impede its realization, are referred to. Despite their relevance for the potential of impact mitigation of to be presented indicators, only an alternative to unsustainable materialist growth is referred to in greater detail in this subchapter. This is because the analyses of the five constraints do not concern the more narrow scope of the research goal of developing indicators of ecological impacts, but rather are relevant for the potential of the indicators to significant mitigation. Thus, only the outcomes of the analyses are presented in the main body of this text and more elaborate investigations of the constraints, particularly of the three topics of external effects, a dilemma of incentives, and the concept of degrowth or post-growth, are placed in the appendix of this work. External effects, also termed externalities, are referred to because they represent the sole currently existing concept that deals with diffuse and non-compensated for ecological impacts, which Kempf (2008) more clearly refers to as the ignored depreciation of the biosphere. Moreover, identification and allocation of effects as achieved in this work are prerequisites for their just and effective 
potential, longer term internalization. It corresponds that due to an increase in environmental issues, for some time the concern of externalities in economic thinking was rising (Uzawa 1999) and still is relevant today. Regarding the dilemma of incentives, despite the motivational conditions of an entity that are assumed under the Prisoners' Dilemma are simplified, it seems that on a regional and particularly on a global scale the difficulty of adoption of sustainability as a vital value in businesses' visions and practices and in governments' policies does resemble the dilemma. One hypothesis is that not competition but the complexity of global markets may be overburdening the intelligence and the senses of responsibility and conscience of the human being. This speaks in favor of the thesis that the human being is more intelligent in being related than in being rational; at least it argues for relational qualities to be of importance. Graeber's finding (2011) that money allows turning moral into an impersonal arithmetic that legitimizes actions that would otherwise be disdained as harmful, supports the former hypothesis.

\subsubsection{The Term 'Sustainability' and Sustainable Conduct}

There is ongoing discussion about the adequacy and usefulness of having extended the scope of ecological sustainability to encompass a social and an economic dimension (see von Hauff \& Kleine 2009 and Ott 2009a). Following Wittgenstein, who said that "the meaning of a word is its use in communication" (Wittgenstein 2001, p. 425), the three-dimensional meaning of sustainability must be viewed as the current state of the art. However, due to the consideration that ecology is the fundament of social and economic action (Reuter 2013), due to the perception of there being more detrimental than beneficial consequences originating from the enlargement, and due to the convincibility of the concept of strong sustainability (Ott \& Döring 2006), in this work the term sustainability solely refers to ecological sustainability. The concepts of efficiency, consistency and sufficiency, with sufficiency being the silver bullet for industrialized countries (Linz 2004, German Bundestag 2013), can be applied to exercise and to strive for ecological sustainability in economic and societal contexts. A more elaborate discussion on the meaning of sustainability is pursued and placed in Appendix A.

Assessing the level of popularity to live sustainability in daily life, results from a biannual study commissioned by the German Environmental Agency (UBA) and the Federal Ministry for the Environment, Nature Conservation and Nuclear Safety (BMU) show that in 2012 nearly two-thirds of the participants call for more intensive engagement of politics in the future wherever linkages between social and economic behavior and an impact on the environment are concerned (UBA \& BMU 2012). This is an increase of eight per cent compared to the results of 2008. However, the percentage of the study of 2010 that indicates the willingness to make changes in one's lifestyle, for instance by 
paying more for environmentally friendlier goods or by sharing material goods like household, gardening, or recreational tools or mobility items, dropped to $50 \%$ and hence shows the discrepancy between what is demanded from others and what people do individually (UBA \& BMU 2010). Over the last twelve years, no clear trends appear. Therefore, a different type of source that may allow assessing current degrees of realization of sustainability and that may further allow forecasting future developments, namely the development of grassroots initiatives is analyzed in addition. Examples of where individual motivation emerges in collective structure and action are numerous, such as, inter alia, community gardens, repair-cafés, community-supported agriculture, time exchanges, local working groups, or petitions. Some activities are connected to or come together under local branches of the international Transition Town Network, which is built around the central ideas of rebuilding community resilience and livelihood and to reduce greenhouse gas emissions, aspiring to live without using much or any crude oil (Transition Network 2013). A concept that was originally developed in agriculture and that supposedly for its explicitness and its higher degree of development and higher potential of implementation and success when compared to sustainability, has received increasing attention is the concept of permaculture. Mollison regards permanent agriculture to be "a valid, safe, and sustainable, complete energy system" (Mollison 1979, p.1), in which species, composition, array and organization of plants and animals are the central factors to serve the welfare and the needs of the people. Mollison points to Fukuoka, in his book The One Straw Revolution, best to state the philosophy of permaculture as, in brief, to work with, rather than against nature, to apply protracted and thoughtful observation rather than protracted and thoughtless labor (Fukuoka 1978). The concept has produced its own ethics of earthcare, peoplecare, limits to consumption and growth, and redistribution of surpluses, and it has a few distinct guiding principles, such as long term instead of short term orientation, diversity instead of monotony, optimizing instead of maximizing, and cooperation instead of competition (Mollison 2010). Despite their presence, no source containing information on the development of the number of people participating in the various forms of grassroots initiatives, which usually have both a social and environmental motivation, could be found.

\subsubsection{Five Constraints to Sustainable Action in Business}

The currently dominating configuration of economic structure, which in most countries of the world and at a global market-level are deregulated market systems, with regard to environmental impact has five major pitfalls: firstly, the aversion to consider external effects, and secondly, the incapability to strive for the joint interest of all people (prisoners' dilemma). Both weaknesses mainly occur due to the inefficiency of too complex structures, non-regulated competition, and a one-sided Hobbesian assumption of the 
human character. The third pitfall is the material growth-paradigm, which in a setting of limited resources and time cannot work infinitely. Regarding environmental impact, the concept disregards that humans are part of earth's ecosystems because in spite of humans' dependence on organic processes and the relational and aesthetical benefits obtained from the presence of diverse life forms, the concept fails to collaborate and instead does away with species, ecosystems, and favorable atmospheric conditions. Fourthly, complex systems of production, the susceptibility to ubiquitous advertisement, and significant differences in pricing, complicate the reflection of purchasing decisions. Depending on degrees of monetary and personal freedom, the latter of which inter alia referring to time, education, and self-determination, before mentioned conditions can significantly impede consumer sovereignty. Fifth, capital sovereignty that establishes through financialization, which is the conversion of the sovereignty of public and private assets, such as social systems, land, water, education, mobility, or information, to the governing of holders of financial products, a process that is prone to happen in neoliberal economies, capital sovereignty according to Kempf (2010) and Foster (2013) means the end of liberal democracy and indicates a condition of oligarchy because the relative autonomy of the state disappears as sovereignty transfers from people to capital. Under this unegalitarian distribution of power originating from self-centered materialist accumulation, nature is likely to be exploited beyond degrees of meaningful sustainability. While all five constraints are explained to greater detail in Appendix $B$, the concept of degrowth is referred to in continuation in the body of this text because it is regarded an essential and promising concept that raises important ethical questions and that is regarded to be inseparable from sustainability and environmental management in industrialized countries.

\subsubsection{The Concept of Degrowth}

For the world as a whole, in 2013 the earth overshoot day, when the human ecological footprint surpassed the biocapacity 3 of the earth, was on August 20 (GFN 2013). Dated

3 The ecological footprint is a measure of the demand human activity puts on the biosphere. Developed in the early 1990's by Wackernagel and Rees, it measures the amount of biologically productive land and water area required to provide the resources and to absorb the wastes of humankind (Ewing et al. 2010). Biological capacity (biocapacity) is the maximum amount of such area that is available on planet earth. It is viewed problematic that the demands of other species are not accounted for in specific. Besides protected sites of restricted human impact, for instance in Germany there exists the wilderness-goal to set aside $2 \%$ of the terrestrial area for ecological processes to take place completely free of anthropogenic control (BMU 2011). Thus, in including the demands humans grant to non-human life forms the biocapacity available to humans would be smaller and the ecological footprint when measured in planets needed would rise. 
even earlier in the year for high income countries, as for instance for Germany, for which the overshoot day already in 2007 was May 16 (GFN 2010), this measure shows how material growth is metabolically unsustainable. The economic theory that deals with the question of how to achieve a socially agreeable and stable, rather than a catastrophic downshifting of an economy, is called degrowth. Kallis (2011) and Paech (2012a) postulate that in a post-growth economy an increase in welfare is possible, that is to live better with less, as for instance regional economic sovereignty will be strengthened, subsistent work will increase resonance and self-efficacy, sharing and exchanging at the local level will raise conviviality, and extreme material inequality will be reduced. The primary goal of degrowth is to establish an economy of a stable throughput that does not exceed ecological limits. This refers to a purely physical concept first introduced by Mill in 1857 as a stationary state (Bladen \& Robson 1965), which was taken up and developed further by Goergescu-Roegen (1973), Illich (1978), and Daly $(1973,1977)$, to from then on be referred to as steady state economy. Schneider et al. (2010), Kallis (2011), and Paech (2012a) enunciate that there exists a second dimension of and motivation for degrowth, which is maybe best described as a cultural or ethical dimension. Latouche (2010) concisely notes that growth did not increase happiness, that credit, advertising, and planned obsolescence maintained a treadmill of production, consumption and growth for growth's sake, binding the creativity, potential for relatedness, and personal quest for the meaning of live, and that the idea of development as growth was corrupting non-Western cultures.

As the normative goal of degrowth is not to degrow GDP, but to reduce material throughput in a socially desirable way, some fields like renewable energy, social services, or organic food produce need to grow. However, degrowth theorists clearly reject framings of qualitative growth, green growth, or green new deal as possibilities to keep current economies of unsustainable throughput running and growing (Brand 2012, Paech 2012a, 2012b).

Regarding the most important principles necessary to allow for socially agreeable degrowth, there is some consensus on implementing a multilevel, confederational, and more direct democracy and redistributing work and leisure, natural resources, and wealth, because a certain degree of equality is one trigger of social welfare and individual happiness (Kallis 2011, Wilkinson \& Pickett 2009), and there is great consensus on the need to relocalize the economy so that most materials, food, energy, and money will be produced and used locally (Paech 2012a, Latouche 2010). As the most important policies a reduction of working hours and a redefinition of work (Haug 2014), taxes, caps, moratoria, and bans for environmentally damaging activities like transport, resource extraction, or energy, as well as on global financial transactions, more sustainable production as in circular flow economic concepts like cradle to cradle or per- 
maculture, a guarantee of minimum healthcare and economic security to all, such as basic income, redistributive taxation, complementary locally based non-interest currencies and depreciative money, support of peasant agriculture, of regional subsistent economic structures, of convivial and relational goods, such as public places or open spaces, and of employment in labor intensive sectors, such as health or education, and significant penalties for advertising are emerging (Kallis 2011, Latouche 2010, Seidl \& Zahrnt 2010, Gorz 2009, Jackson 2009). The change of societies' basic institutions, such as financial, property, political, and redistributive, which have come to depend on and to mandate economic growth, is viewed to be particularly difficult.

While for the concept of degrowth soon to become societies' vision and politicians' agenda seems highly unrealistic, degrowth is not as far-fetched or romantically idealistic as it may seem. Many of the ideas and policies referred to above already are partially being implemented, such as carbon taxes in Ireland, moratoria for nuclear energy or fracking in Germany, a reduction of working hours in France, financial transaction taxes, regional currencies, community supported agriculture, and local time exchanges. Others are discussed in working teams both at the local and national level, such as basic income (Werner 2008) or alternative welfare indicators (German Bundestag 2013). It seems realistic that for a transition, developments and contributions from both local economic and democratic empowerment and action and from national political action would play an important role (Hopkins 2008).

Ending his book Liberation from Excess, Paech either consciously or unintentionally refers to the central question in ethics, which was also put forward in section 2.2 of this text, namely how we should live. Asking if one can be truly happy with things that with regard to personal conscience of global welfare one cannot vindicate to possess or use, relating to both eudemonia and morale the author concludes that enlightened happiness would require not only to enjoy, but also to be at peace with one's conscience. Degrowth and post-growth economies could form a favorable foundation to this end (Paech 2012a). The perception of the individual that using and having less does not mean being less, but that simplicity fosters one's own self-development, happiness, and peace, and that it is a morally consistent act of solidarity seem to be the strongest levers for individual and collective efforts to emerge and to establish societies characterized by organization and aspiration of post-material growth (cp. Burch 2009). In order realistically to assess the potential of mitigation of the ecological impacts concerned by to be presented indicators, the related phenomenon of highly unsustainable material throughput, which at this stage implies an unsustainable intensity of transport, and the alternative concept of degrowth briefly were set forth. A more detailed description of the reasons viewed to speak in favor of the concept and the concept's multidimensional reach is given in Appendix $B$. 


\subsection{Summary and Conclusion}

In section 2.1, the biological conditions and the development of the political consideration of the human induced crisis of biodiversity are referred to. Biodiversity is the variety of life in all its forms. Research has revealed that ecosystems and biodiversity over the last 50 years changed and went extinct at a pace unprecedented in human history. Land use, climate change, overexploitation, invasive alien species, and pollution were identified as the five main direct threads to biological diversity. The increasing implementation of national strategic plans as required by the CBD, and the foundation of the 'Intergovernmental Platform on Biodiversity and Ecosystem Services' are indications of the current relevance of the subject. Due to the three major environmental impacts of land use, greenhouse gas emissions, and fragmentation, road transport is one activity that significantly contributes to the alteration and deterioration of habitats, ecosystems, and earth systems. In the research field of road ecology, the effects of roads and vehicles on their environment are analyzed in order to facilitate avoiding and mitigating undesired negative impacts. The scientific basis of the assessment of impacts described in Chapter 4 predominantly originates from studies from this field.

The question, why impacts could be rated undesirable, is pursued in section 2.2. To this end the relation between humans and non-human nature is pondered. It results that the central question in ethics, how we should live, in part must be answered: "allowing for other life forms to be around". Krebs names eudemonic and moral values as the two major ends to orient one's actions towards. The two categories resemble the two prominent concepts of instrumental or moral valuation of nature, anthropocentrism and physiocentrism. It is found that while the distinction between the two groups of values and the denial or granting of innate value to non-human life forms is adequate, the frontiers are 'soft' as adhering to moral values can also be eudemonic and as people who exclusively advocate anthropocentrism or physiocentrism are rare. Nussbaum found the instrumental and relational value of nature in many cases to be essential to live a flourishing life. She thus defined nature's healthy presence to be one of humans' ten minimum capabilities to be granted by societies. For Becker, the role of nature in part is answered by his conception of the human being as a fundamentally dependent and relational being, holding three fundamental relationships to contemporaries, to nature, and to past and future generations. Assuming virtue ethics and ethics of care, he views the reduction of the human being as exclusively rational, the assumption of autonomy and the growth paradigm as ultimate ends, and the increasing complexity of most of life's meta-structures as major hindrances to live as a relational, sustainable person. The philosophical arguments presented in this text speak in favor of the existence of non-human life and they are viewed to be adjuvant to answer the question, to which ends the research goal of improving environmental impact assessment is set. 
In section 2.3, the term sustainability best to refer to an ecological dimension alone, and the absence of a clear trend in sustainable conduct in society are found. The analysis of a missing realization of sustainability by corporate players reveals five structural economic constraints: first, despite underlying rules and regulation, in market economies the interests of activated players are predominantly recognized. As the environment is a rather passive player, activated by human valuation to some extent, it suffers from external effects to a high degree. Yet, it is concluded that like in the case of the hypothesized motivational dilemma of the inefficient use of public resources, the competition in markets in most cases does not force players to act in a certain way. Material growth is considered to be a third structural economic constraint. While growth is not prejudicial per se, growth sought due to structural imperatives, which do not serve the dignified well-being of all people, is to be declined. For the degradation of 'natural resources', that is materials, ecosystems, and geophysical systems, and for the recognition that material wealth and happiness are not the same thing, the growth mandate inherent to the economic systems of all industrialized countries at the beginning of the $21^{\text {st }}$ century, is rejected by the economic concept of degrowth. While it is difficult to choose the most central characteristics of the multi-dimensional concept, the goals to establish an economy of stable throughput that does not exceed ecological limits, a multi-faceted and locally empowering democratic structure, a partial demonetization and regionalization of economic structure, a positive perception of sufficiency, and a higher relatedness to things, people, and non-human nature, are meaningful aspects that can be named. In short, degrowth is the transition to a state of living better with less. A decrease of consumer sovereignty and an increase of capital sovereignty are identified as two further constraints.

In Chapter 3, methods currently used in environmental management are analyzed in order to screen, if ecological impacts of road transport are sufficiently acknowledged and mitigated. 


\section{Methods Used in Environmental Management}

Companies at different stages of supply chains are concerned about the environmental impacts of their products because their business customers pass on demands from customers and from regulation. A company selling to end-customers does no longer limit its focus on their products or the in-house production process, but it extends the view to the supply chain. The relevance of environmental management is still growing (cp. Matthews et al. 2004). It increased quickly, as corporate environmental management developed from isolated examinations to a systems approach. Despite regulatory or monetary incentivizing installations, for instance like the European Emission Trading Scheme (EU-ETS), end customers' demands usually are the strongest driver behind a company's environmental management efforts (Christini et al. 2011). There are two principal reasons for extending environmental management to supply chains: firstly, while originally evoked to a great extent by scandals of child labor in the apparel industry, the level of customer sensitivity for all practices accepted by a final producer has risen, and secondly, the most efficient and quantitatively most meaningful reductions of environmental impacts were detected often to lie outside a focal company's premises. Land use changes from raw material production or negative impacts from the usage or disposal of a product are typical examples of substantial impacts occurring at different stages of a product's life cycle. In order to identify potential shortcomings, in this chapter, existing environmental management tools and standards are analyzed regarding their consideration of impacts on biodiversity and their consideration of ecological impacts of road transport, and here of land use and landscape fragmentation in particular.

Besides corporate and other organizational entities, also communal and governmental entities, from local committees to federal ministries, use methods of environmental management. A few selected methods will be referred to briefly in the second part of this chapter in order to assess if they can be of use for corporate environmental management. For the same reason, in a third subchapter, the methodologies, trends, and results of indicators that measure the overall impact of land use and fragmentation at the level of very large areas and for the totality of human activities, will be turned to.

\subsection{Corporate and Organizational Tools of Environmental Man- agement: LCA, EMAS, ISO, and GRI}

Primarily for the development that consumers hold end-producers responsible for all impacts associated with the production, use, and disposal of their products and services, as well as the awareness of companies that for efficient improvement of environmental impacts it makes sense to exceed a focal company's premises, life cycle 
assessment (LCA) became the prevalent technique to assess environmental impact. Management systems demand or recommend for LCA to be used. As indicated by the name, in LCA environmental impacts associated with all stages of a good's life, that is from-cradle-to-grave, are investigated. LCA traditionally was used in strategic decision making in order to monitor the significance and the shifting of environmental impacts in production systems (Ridoutt \& Pfister 2013). While LCA existed and was used for a much longer time, it received a new wave of attention when the demand to check products for greenhouse gas emissions set in. Due to the new relevance of carbon footprinting, LCA results had to address wider audiences. On the one hand this bore the risk of disregarding the core LCA principle of comprehensiveness (Finkenbeiner 2009), but on the other hand the elevated demand had the potential to challenge and to promote current LCA frameworks (Weidema et al. 2008). For instance to group impacts into footprint families like greenhouse gases, land use, or toxicity, to enable users to weight certain impacts, or to better account for the impacts on ecosystems and natural capital could improve the description and application of impacts and results (Zhang et al. 2010). With regard to determining impacts on biodiversity, life cycle assessment and environmental management overall face the issue that its measurement is complicated and not yet standardized. While a theoretical agreed on definition on what biodiversity is exists, even at the definitional stage questions on the preferable abundance within species and on the size of ecosystems remain. For instance assumed under the most ambitious environmental management tool, the Sustainability Reporting Guidelines G4 (G4) of the Global Reporting Initiative (GRI), the two central impact categories for biodiversity are effects on protected areas and on areas of high biodiversity value. The biodiversity value of a site usually is high for its species diversity or abundance and for the scarcity of species or ecosystems.

The topic of biodiversity found its first noteworthy incorporation in a life-cycle assessment tool in 2006. While the eco-indicator 99 does not refer to biodiversity and the CML model from 2001 only mentions but fails to conceptualize it, the Global Reporting Initiative in its 'Sustainability Reporting Guidelines G3' in 2006, firstly and explicitly formulates two core, and three add-on indicators on biodiversity (Pré Consultants 2001, Guinée et al. 2001, GRI 2006). The higher goal of the GRI is to make sustainability reporting standard practice. Until this state is reached, the guidelines predominantly function to help companies and organizations to determine and to report the environmental impacts of their processes and products' life cycles. The environmental aspects considered in the G4 are: materials, energy, water, biodiversity, emissions, effluents and waste, products and services, compliance, transport, overall, supplier environmental assessment, and environmental grievance mechanisms (GRI 2013). While the ecoindicator 99 and the CML model are life cycle assessment tools, today the two most 
popular environmental management systems used by organizations for certification and in order to help reduce environmental impact in a systematic way are the ISO 14001:2004 (International Organization for Standardization) and EMAS III (Eco Management and Audit Scheme) standards.

Besides the ISO 14000 series, which has special norms for the assessment of greenhouse gas emissions at the organizational level (ISO 14064-1:2012-05) and at the product level (ISO/TS 14067:2013-05), ISO has developed a guidance document for corporate social responsibility, entitled ISO 26000:2010 (ISO 2011). In the 26000 series the environment is also referred to and the protection of biodiversity and of ecosystem services are viewed as two means of conducting more social-responsible business processes (B\&B Campaign 2011a). However, the ISO 26000 document cannot be used for certification as can be the ISO 14000 series. The ISO 14000 norms do not establish absolute requirements for environmental performance; rather they are intended to provide organizations with the elements of an effective management system. The following five constantly recurring phases represent the core of the system: 1 . an organization's environmental policy must be defined by top management; 2 . in the planning phase environmental aspects of its activities, legal requirements, objectives, and environmental programs shall be identified; 3. in the implementation and operation phase responsibilities, training, communication, documentation of the system, and operational control shall be organized; 4 . in the phase named checking and corrective action significant environmental impacts of activities shall be monitored and measure and audits be carried out; 5 . top management at intervals shall check the suitability and effectiveness of the entire system (ISO 1996, DIN 2012). However, the EMAS III requirements appear to be more demanding than those of the ISO 14000 series, because under EMAS the requirements to determine specific significant impacts, to comply with additional management elements, and to continually improve environmental performance, seem more binding (EMAS 2011a, 2011b).

While ISO $14000 f f$ only implicitly refers to biodiversity, in EMAS III, biological diversity is one of the eleven most important environmental aspects (DIN 2012, EMAS 2009). Moreover, biodiversity is classified as one of six environmental core indicators and it is represented by the impact of land use (EMAS 2010). However, neither of these two management systems touches on biodiversity as detailed as the GRI standards G3, G3.1, and G4, which is part of the reason why the GRI standards became the international benchmark for sustainability reporting (GRI 2006 \& 2011, Gray 2006, Adidas AG 2013, Bauer 2013). In the latest version of guidelines G4, the aspect of biodiversity is referred to by one "guidance-entry" (G4-DMA), which demands for the Disclosure of the Management Approach, which means to describe an organization's strategy for applying its policy and achieving its goals of biodiversity management, and by four specific 
indicators (G4-EN11 to 14), which demand information on operational sites in or adjacent to protected areas and areas of high biodiversity value (G4-EN11), a description of significant impacts of activities, products and services in such areas (G4-EN12), information on habitats protected or restored, which refers to the prevention and redressing of negative impacts associated with activities (G4-EN13), and information on cases where IUCN Red List or otherwise classified endangered species are threatened by activities related to an organization (G4-EN14) (GRI 2013).

Companies that want to get a first overview of their impacts on biodiversity can benefit from checklists developed and provided by several NGOs, for instance the Business \& Biodiversity Campaign or the Business in Good Company initiative (B\&B Campaign 2011b, Business in Good Company 2011). Different sets of checklists for all functional units exist, mainly focusing on the first step of the identification of a company's existing impacts. Concerned in the checklists are all areas where a company has direct control, or where it at least has substantial influence on processes carried out by other entities within the supply chain or within the life cycle of a product. Although the questionnaires allow for both a comprehensive and detailed assessment, they are not yet elaborated and standardized enough to function as management systems. However, their strong point is that they are tested and developed in collaboration with organizational and corporate users and hence can give serviceable input and guidance to the formulation of standardized management systems and certifications.

Regarding the consideration and management of impacts from transport, the EMAS regulation of 2009 (Regulation EC No 1221/2009) mentions traffic as one of eleven direct environmental impacts that are mandatory to be assessed and managed by an organization. However, no more detailed explanation of the potential environmental impacts caused by different modes of traffic are made so that the aspect of guidance is weak and the quality of the requirement is low because no detailed or comprehensive analysis is demanded (EMAS 2009). In the various norms of the ISO 14000 series, traffic is not specifically mentioned, which primarily is because ISO predominantly follows the approach of eco-balancing and its two respective parts of life cycle inventory and life cycle impact assessment. However, apart from the greenhouse gas effect of emissions, four further effects of transport, namely land use or habitat loss, landscape and habitat fragmentation, invasion of neophytes, and altered states of competition through substance emissions are listed as non-compulsory results of a possible life cycle impact assessment in norm 14025 2011-10 (DIN 2012). While emissions into the air and land use are indicated as potentially relevant environmental aspects in norm 14004 2010-08, fragmentation and neophytes are not. Impacts from traffic neither are referred to in an elaborate, exemplary list of suggested key performance indicators in the same norm (DIN 2012). Overall, both tools, EMAS III and ISO $14000 f f$, lack a com- 
prehensive assessment and management of ecological impacts from transport. Emphasis remains on energy demands and emissions. Analyzing sustainable manufacturing, Chun and Bidanda (2013) also ascertain missing comprehensiveness.

Also with regard to transport the GRI standard seems to be the leading instrument. Compared to EMAS, it extends the demand to report the significant environmental impacts of transport not only for materials and products, but also for members of the workforce (GRI 2013). In addition, the GRI standard seems to be clearer and more ambitious in its requirements, which include the demand to report how impacts from transport are mitigated, by which criteria and methodology impacts were determined to be significant, and what were reasons in cases in which quantitative data is not provided (GRI 2013). However, while also the estimation of the relevance of environmental impacts of transport systems is well explained, 4 the proposed groups of impact, including energy use, emissions (e.g. GHG emissions, $\mathrm{NO}_{x}, \mathrm{SO}_{\mathrm{x}}$ ), effluents, waste, noise, and spills, only in the impact of noise go further than do EMAS and ISO 14000ff in their requirements (GRI 2011). One difficulty of the GRI guidelines is that their good reputation can be misused because the Global Reporting Initiative does not control, if its guidelines were fully or sufficiently adhered to. For instance, while Adidas AG says to have applied the GRI guidelines as of version 3.1 to their 2012 sustainability report, the disclosure is not intuitive with regard to environmental impacts as it is structured different to the GRI guidelines and also is far less exhaustive than the guidelines demand (Adidas AG 2013).

Overall, the two most recognized environmental management standards that certify, EMAS III and ISO 14000ff as well as the leading guidelines tool, the GRI G4, do not require or recommend comprehensive reporting and management when it comes to the impacts of road transport. Energy demands, greenhouse gas emissions, and other emissions are being focused upon, while land use, fragmentation, and invasive alien species are insufficiently considered. This is reflected by corporate efforts. Companies that are recognized to have implemented sustainability as one of their core principles, attempt to reduce greenhouse gases with much ambition as they for instance assist employee commuting in order to reduce motorized mobility but seldom extend their management to further impacts (Memo AG 2013, Vaude 2013). Only very few compa-

\footnotetext{
4 "The environmental impacts of transportation systems have a wide reach, from global warming to local smog and noise. For some organizations, particularly those with extensive supply and distribution networks, environmental impacts associated with logistics may represent a major part of their environmental footprint. Assessing the impacts of transporting products, goods, and materials for logistical purposes, and transporting members of the organization's workforce, is part of a comprehensive approach to planning environmental management strategies" (relevance of the environmental impacts of transport as in G4; GRI 2013).
} 
nies like the BMW Group mention land use and noise as environmental impacts being produced by their road transport activity (BMW Group 2013). Yet, also here 'management' is not the correct term to be used because they do not move beyond the step of recognition and do not offer quantifiable indicators. Hardly any company was found to refer to other ecological impacts like fragmentation or invasive alien species. The only company that reported on noise elaborately and indicated numeric data is Deutsche Post DHL (DHL 2013).

With regard to software tools, the eco-balancing tool 'eco-indicator' in its current version number three does calculate land use for transport processes, but does not refer to fragmentation (Ecoinvent 2013). Regarding land use, the tool appropriately calculates impacts according to vehicle weight, but in the method of calculation it is not differentiated by road type, which decreases the accuracy of allocation of impact (Spielmann et al. 2007). While the lack of methods of quantification is not viewed to be the major constraint to setting greater store by the ecological impacts of land use and fragmentation from road transport, their missing identification, communication, and management are facilitated by the absence of quantifiable methods in environmental management tools and standards. Thus, in Chapter 5 of this work, an alternative and more accurate calculation of an individual road user's direct land use impact as well as two indicators that allow a measurement of the wider land use impact of negatively affected area adjacent to roads and the impacts of landscape and habitat fragmentation are introduced.

\subsection{Environmental Management Tools Used by Public Authori- ties: EIA and SEA, Ecological Risk-Analysis, the DPSIR- Model, and the Precautionary Principle}

Apart from the DPSIR-model $(D=$ driver, $P=$ pressure, $S=$ state, $I=$ impact, $R=$ response), the environmental management tools introduced in the following paragraphs are not limited to, but clearly have a site specific, spatial and land use planning focus in assessing environmental impacts of land use. While they are often used in cases of public interest, such as mobility concepts and construction of infrastructure, waste management plans, energy concepts, regional development concepts, or tourism concepts, private organizations can be concerned when they want to operate or build plants in natural sites.

The strategic environmental assessment (SEA) is a systematic investigation procedure required to be conducted by law in Europe in order to consider environmental aspects in the strategic planning of policies and programs of public authorities, and at times of 
private bodies. In the European Union the procedure is formulated by the directive 2001/42/EC (EU 2001). Usually, in addition and in a phase prior to the strategic assessment, an environmental impact assessment (EIA) is conducted. The idea of this mechanism is to analyze and test potential environmental impacts before projects are licensed. This is because some fundamental impacts are evident already in the first phases of planning. When impacts are analyzed in an EIA at the planning level, after the approval of a project the same aspects usually do not need to be re-examined in a SEA. No standards exist that the results of an EIA have to adhere to or that would require specified actions. Rather, it compels decision makers to account for environmental values in their decisions and to justify those decisions in light of detailed environmental studies and public comments on the potential environmental impacts (Holder 2004). An EIA consists of seven key areas: description of the project, alternatives that have been considered, description of the environment, description of the significant effects on the environment, mitigation, non-technical summary, and lack of knowhow/technical difficulties (EU 1985). The directive on environmental impact assessment of the European Union, originally 85/337/EEC and since $20112011 / 97 / E U$, has to be implemented in the laws of the member states, as for instance done in the Law on Environmental Impact Assessment of Germany (Gesetz über die Umweltverträglichkeitsprüfung - UVPG), which explains the directive and issues a list of projects of different sectors that fall under the law (BRD 1990, last amendment July 2013).

A quantitative instrument that is used in environmental impact assessment during the planning period of a project is the Ecological Risk Analysis. While the resolution of the National Environmental Policy Act (NEPA) of the United States in 1969 marks an even earlier milestone in environmental impact assessment, the ecological risk analysis as developed in the late 1970 s contributed much to the compilation of the first European EIA directive in 1985 (Aulig et al. 1978). The central procedure of this method is to interrelate the intensity of potential impairments produced by the originator to the susceptibility of the respective ecological systems, the result of which is the determined ecological risk. The intensities of environmental disturbance and the sensitivities of the ecological systems are usually clustered in relevance trees, argument tables, and preference matrices (Bechmann 1998). This procedure allows assessing impairment not only to be significant or negligible, but requires a more detailed analysis of the damage of the impact. The target of the method was to allow for an assessment of ecological agreeability of land uses under incomplete information. The method does not imply a mandatory or tightly defined procedure of assessment. This stems from the basic original motivation to develop a methodology that would oppose increasingly economically driven politics as well the claim that long-term ecological and social impacts were too vague and hence not eligible to be considered in project planning. Thus, the formula- 
tion and communication of the guiding principle, in the beginning may have been more important than the definition of strict guidelines of quantification. Ecological risk analysis for instance is used for environmental impact studies in traffic and transport planning (Aulig et al. 1978, Bechmann \& Hartlik 2004).

The fourth method of human impact analysis on the environment referred to here is the DPSIR-model. In contrast to the two procedures and the instrument mentioned above, the DPSIR-model usually has no site specific focus, but evaluates impacts in broader contexts of cause and effect that concern larger geographic areas like regions, nation territories, or the whole world. Based on the PSR-concept devised by the OECD in the late 1980s, the European Environmental Agency (EEA) developed the model further. It now consists of five elements that are related to each other in a chain of cause and effect. The goal was to create a systemic methodology that related the reasons for environmental disturbances, biological change, and potential preventive action in a causal chain and that would be able to take into account concomitant influences of socioeconomic developments.

The functioning of the method can best be illustrated by analyzing the five phases, which are driving forces, pressures, state, impacts, and responses, in an example. Here, the use of land in South America in order to produce animal feed imported by Germany is constructed. Driving forces are German consumers, who demand increasing quantities of animal products at low prices, animal product companies, which decide to buy fodder from South America and which advertise and export their products to earn more money, and rivalling interests of land use in Germany. Pressures are the demands for land in South America in order to cultivate the crops. The state is that in South America land existed before the demand arose and that the land used to serve other interests like the growing of trees and the living of all other organisms in those trees. The state hence is the amount of land available to forests and the amount of land available to agriculture. No other land is available. The impact is that in order to grow more crops, forest land is converted into agricultural area so that the quantity of agricultural area increases and the quantity of land available to forests decreases. The societal response in Germany might be that the high consumption of animal products leads to research that finds certain degrees of consumption of animal products to be unhealthy or not necessarily essential for the physical well-being of the human being. In addition, consumers learn about the consequence of forest being lost. This to them may create a conflict because they also like forests and because they feel uneasy about the fact that their food comes from so far away and that despite being voluntarily offered to them they use large amounts of soil in regions, whose culture and history they do not know. This response in research and thought, in the scenario at hand may 
lead to consumers' decisions to use less animal products. This equals a change in driving forces and an alteration of all elements in the causal chain of effects.

Since the method normally is used by political institutions, the 'response' typically is a certain policy that can affect any or several of the other four elements of the chain. In the example above, techniques to improve crop yields would have lowered the pressure; the making available of land of a different use type would have changed the state. The strong point of the method is the possibility to analyze and estimate, if certain policy responses will lead to reduced impacts or if they will not. For instance in the example of fodder, it may be that the amount of reduced demand for animal products in Germany means that this amount is now exported to other people. If the goal was the absolute reduction of land being used for crops, the goal would not have been reached. However, in the above case, it is much more likely that the goal would have been for the German person to demand less land in South America, a goal that for the latitude of the individual person is very likely to be reached. While SEA, EIA, and ecological risk analysis focused on the determination of environmental impact, environmental impact remains the central motivation in the DPSIR-model. However, not its determination, but rather the original reasons (driving forces) and most of all the assessment of opportunities to change an impact through societal reaction or policy (response), are the core features of the DPSIR-model. Despite the complex scope of the DPSIR-model it seems to be of higher value to corporate or organizational entities than the other three measures because they require degrees of environmental expertise and manpower improbable to be hold by many entities and because due to their site specific focus they seem more relevant to entities with land use intensive activities, such as resource extraction, renewable energy production, or transport intensive activities.

The Precautionary Principle is no discrete methodology. Rather it is a guideline that is recommended to be used in impact assessments and the estimation of ecological responses. It reflects the condition that in many cases mankind can make more or less probable predictions, how natural systems will be affected, or how they will react to human impacts, but that in many cases there remains a significant degree of uncertainty. Two reasons of uncertainty are the high complexity of ecosystems and the knowledge gap of the existence and description of the estimated 8.7 million eukaryotic species on planet earth, of which about $86 \%$ on the earth and $91 \%$ in the ocean still await description (Mora et al. 2011). Applied to the loss of biodiversity, this means that the humanly provoked reduced abundance and extinction of any species potentially has greater ecosystem consequences (Sala et al. 2000). Another dimension that constitutes the precautionary principle is time lags in ecological responses. Consequences of impacts on ecosystems or species can become apparent only after decades. In the case of biodiversity loss this phenomenon is called extinction debt (Tilman et al. 1994). 


\subsection{Aggregate Landscape Indicators of Land Use and Fragmenta- tion}

In this section, the conditions and trends of land use and fragmentation, as well as the methods used to quantify both impacts at large geographical levels, are be presented. The focus will be on two methodologies that measure fragmentation, namely 'nondissected low traffic areas larger $100 \mathrm{~km}^{2}\left(\mathrm{UZVR}_{100}\right)$ ' and effective mesh size ( $\left.\mathrm{m}_{\text {eff }}\right)$, because they are more complicated and less intuitive than is the differentiation and calculation of types of land use. In Article III an investigation of landscape level fragmentation is carried out for the Biobío Region of Chile and here presented methods of quantification are applied.

Land use claims, sometimes also entitled 'change of habitat', that occur for instance for agricultural expansion, resource extraction or an extension of areas of settlements and transport infrastructure, have a substantial negative impact on biodiversity and ecosystems (van der Ree et al. 2011). The major negative effects from sealing natural surfaces are loss of the functions of the natural soil, loss of fertile arable area, and loss of ecologically intact area, including its biodiversity (BMU 2010). Despite being expected to be displaced by the effects from climate change in the future, land use change or degradation is viewed still to create "the biggest single source of pressure on biodiversity" (CBD 2010a, p. 55). While land use is the anthropogenic expression, it translates into habitat loss and degradation, when the planet's natural life forms and ecosystems are concerned. Pressures on natural lands are a worldwide phenomenon. The largest amounts of habitat loss continue to occur outside of Western societies and in regions nearer the equator, where forests or wild lands are usually converted to agricultural area, recently increasingly serving biofuel production. However, also impacts from urbanization and infrastructure development, such as housing, industry and transportation networks provoke daily land use claims to reside at unsustainable levels also in industrialized countries. This is predominantly valid for Western countries, but also for economically developing countries like various states in Eastern Europe and for parts of China and India (CBD 2010a). Here, an exemplary overview of the condition of land use in Germany is given. It is legitimate to assume similar tendencies also in most other industrialized countries, because infrastructural development was found to correlate with gross domestic product (Jaeger et al. 2005a, Federal Statistical Office 1999).

In Germany, land use is listed under the name 'area for settlements and transport infrastructure'. The percentage of area for settlements and transport infrastructure that is sealed is estimated to be between $43 \%$ and $50 \%$ (Federal Statistical Office 2012). At the end of 2012, area for settlements and transport infrastructure used up $13.5 \%$ of Germany's total land mass. Between 2009 and 2012 the area consumed increased by 
$2.3 \%$. That equals a daily claimed area of 74 hectares or approximately 106 soccer fields (Federal Statistical Office 2013b). Compared to the previous four year period between 2008 and 2011 the daily consumption came down 7 hectares, from before 81 hectares per day (Federal Statistical Office 2013a). This continues a positive trend in demanding less new land since 2004. In spite of the overall average yearly decline only being 2.7 hectares per day per year, that is calculating from 1996 onwards when the absolute average value was 120 hectares per day, there can be slight hope that the official 30 ha target will be reached by 2020. The target exists since 1998 and has recently been confirmed as part of Germany's National Biodiversity Strategy (BMU 2010, BMU 1998). Subtracting the area needed for uses like housing or industry from the total area of $13,5 \%$, the area consumed by transport infrastructure results to account for $5.05 \%$ of Germany's entire land mass (Federal Statistical Office 2013b). Whether this means that infrastructure and mobility are a luxury depends on how valuable natural landscapes or rival interests of use like agriculture, forestry, or natural resource extraction are viewed.

For an overview on the extent and the impacts of fragmentation, the study 'Landscape fragmentation in Europe' of the year 2011, which was presided by the European Environmental Agency, is referred to. Studies in other industrialized countries have revealed similar results, as for instance in the case of the USA (Forman et al. 2003). The European study points out that fragmentation also is a significant issue in not yet fragmented regions, where the pace of development lead to a relatively strong further increase in transport infrastructure. Particularly negative were the effects on biodiversityrich, mostly natural and largely undisturbed areas (EEA 2011). Figure 3.1 shows results of the European study on fragmentation by country. The method that indicates levels of fragmentation is effective mesh size $\left(m_{\text {eff }}\right)$. It is explained in detail on the following pages. Particularly Scandinavian and Eastern European countries dispose of less fragmented landscapes. Yet, the study points out that seemingly lower levels of fragmentation should not be judged in the light of the values for central Europe, because these were among the highest in the world. On the contrary, the study recommends not repeating the mistakes made in central Europe because their avoidance would favor the conditions of biodiversity and also save respective countries large costs. Such costs currently occur for instance in Germany for attempts of defragmentation.

Another approach that is used to measure fragmentation is the summation of large undissected areas. Coming from a value of $26.5 \%$ in 2000 , in Germany, the percentage of non-dissected low traffic areas larger $100 \mathrm{~km}^{2}\left(\mathrm{UZVR}_{100}\right)$ in 2005 fell to $25.4 \%$ (BMU 2010). Also this method is explained in detail on the next pages. While the goal was for the value not to decrease further than $25.4 \%$, already in 2007 in the National Strategy 
on Biological Diversity the value was assumed to have decreased to about 23\% (BMU 2010, BMU 2011). The most recent numbers are of the year 2010 and they disclose the total area of non-dissected low traffic areas larger $100 \mathrm{~km}^{2}$ to account for $23.16 \%$ of Germany's land mass (BfN 2013).
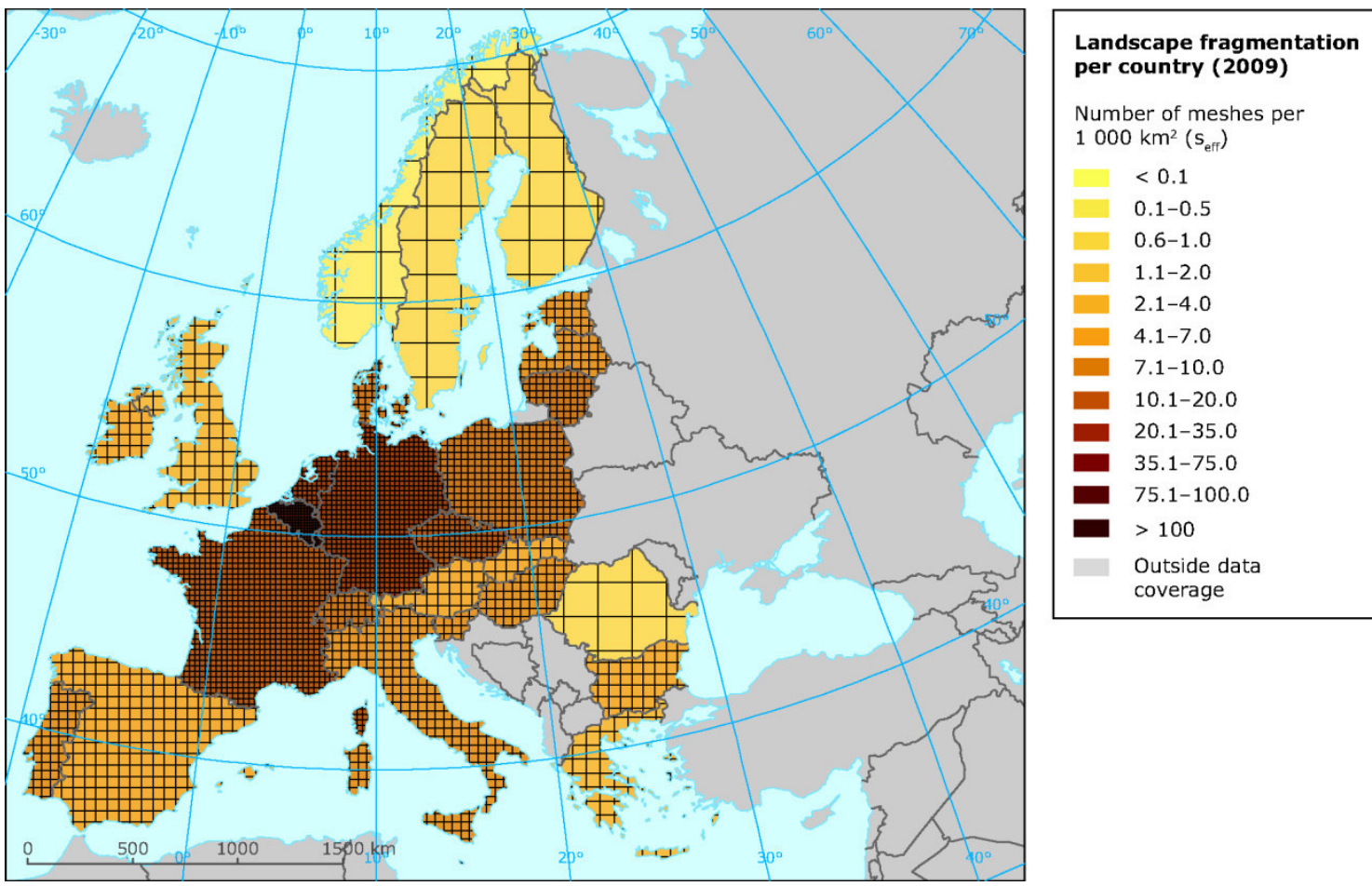

Figure 3.1: European levels of landscape fragmentation by effective mesh size (Use with permission; EEA 2011).

A recent and popular example underlines the worldwide relevance of the ecological problems deriving from fragmentation. In 2010, plans of constructing a tarred highway across the Serengeti in Tanzania surfaced. The objective was to foster economic development and trade with Uganda. Protests from environmental agencies, Tanzanian Tourism agencies, and the consideration of the UNESCO then to put the famous Serengeti National Park on the Red List of endangered World Heritage Sites were the response, because degradation beyond repair of this valuable ecosystem was predicted. In June 2011 the Tanzanian government withdrew from the plan and the road to be constructed will in the east end in Loliondo and in the west in Mugumu. Roads within the national park will not be tarred and remain under the authority of the park administration (Greenpeace 2011).

Over the last twenty years, two methods prevailed to measure the degree of fragmentation within a landscape. Both approaches are useful in spatial planning and they also can serve businesses to determine patches of landscape that are regarded ecologically valuable due to being considered undissected and large in size. However, they are not 
apt to serve an individual business as an indicator to actually measure its fragmenting effect. The first measure to be turned to is that of 'undissected low-traffic areas larger $100 \mathrm{~km}^{2}$ ' (UZVR $\left.{ }_{100}\right)$. The method was developed at the Federal Agency of Nature Conservation of Germany (BfN) in the 1980s (Lassen 1979, 1987). Undissected low-traffic areas larger $100 \mathrm{~km}^{2}$ by definition need to have a minimum size of $100 \mathrm{~km}^{2}$ and they cannot be dissected by a road with a traffic intensity greater 1,000 vehicles per day, by railways of two or more tracks or electrified tracks, by a waterway higher than category 3 , by airports or built-up area, and there cannot be tunnels shorter than 1,000 meters or water bodies that are larger than half of the total area (Schupp 2005). Strengths of the UZVR-approach are that it is easy to comprehend as it is clear and illustrative in its results. It also is regarded to be of ecological quality because undissected areas provide an important basis for regional, national, and supranational ecosystem functioning and the diversity of flora and fauna (Turner 2006, Selva et al. 2011). Deficiencies of the approach are that it possibly favors large areas over smaller, but ecologically more valuable areas, as size alone yet is no proof of a valuable natural ecosystem. Furthermore, it can be questioned why exactly $100 \mathrm{~km}^{2}$ and 1,000 vehicles per day and no smaller or larger values are used in both criteria. In total, the benefits of this approach seem to outweigh its limitations, also because people working in the respective agencies are usually well aware of its weaknesses (BMU 2010).

The second internationally well-established fragmentation measure is called effective mesh size $\left(m_{\text {eff }}\right)$ (Jaeger et al. 2006). It relates to each other the size and the multitude of undissected areas within a total area, so that the result can function as a quantitative expression of landscape connectivity (Reck et al., 2008). Since it takes into account the possibility of movement between all points in a given landscape, it expresses the probability of two randomly situated animals to meet each other. It hence considers the most basic prerequisite for the persistence of animal populations (EEA, 2011). The fragmentation geometry, that is the rules for natural or artificial features of the landscape to be considered as boundaries and edges of patches, can be determined by the person applying the method. When an UZVR-value and $m_{\text {eff }}$ are calculated for the same region, fragmentation geometries usually are identical. In order to obtain a final result of the effective mesh size that can be compared to other areas, the probability calculated for two animals to coincidence is multiplied with the size of the total area

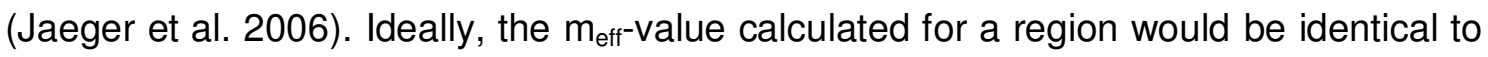
the size of that region. This would mean that no fragmenting elements existed and that from any point in the landscape all other points could be reached. Only in the case of all patches being of identical size does $m_{\text {eff }}$ yield the average size of all patches. Particularly in comparison to the fragmentation measure of undissected low-traffic areas, one advantage of the $\mathrm{m}_{\text {eff }}$-measure is that it analyzes a region in its entirety, as habitat 
patches of all sizes are considered. The $m_{\text {eff }}$-approach is depicted by the formula depicted below. It was first published by Jaeger in 2001.

Equation 3.1: Formula of the fragmentation measure 'effective mesh size ( $\left.m_{\text {eff }}\right)$ '.

$$
\mathrm{m}_{\text {eff }}=\left[\left(\frac{\mathrm{A}_{1}}{\mathrm{~A}_{\text {total }}}\right)^{2}+\left(\frac{\mathrm{A}_{2}}{\mathrm{~A}_{\text {total }}}\right)^{2}+\left(\frac{\mathrm{A}_{3}}{\mathrm{~A}_{\text {total }}}\right)^{2}+\ldots+\left(\frac{\mathrm{A}_{\mathrm{n}}}{\mathrm{A}_{\text {total }}}\right)^{2}\right] \cdot \mathrm{A}_{\text {total }}=\frac{1}{\mathrm{~A}_{\text {total }}} \sum_{\mathrm{i}=1}^{\mathrm{n}} \mathrm{A}_{\mathrm{i}}{ }^{2}
$$

$A_{i=1, \ldots, n}=$ sizes of the individual areas or patches $i$ with $i=1,2, \ldots, n$

$A_{\text {total }}=$ total size of the investigated region

One detail of both the UZVR-method and $m_{\text {eff }}$ that needs special attention is the definition of the 'total area'. Two approaches are thinkable: either to assume the entire land mass, including for instance inland waterbodys or areas occupied by cities, or to assume only the natural area apt as habitat for the species concerned in a given analysis. Although both measures focus on analyzing degrees of fragmentation, for UZVR usually the entire land mass is assumed as the total area (e.g. BMU 2010), whereas for $m_{\text {eff }}$ only the sum of all identified habitat patches is used (e.g. EEA 2011, Jaeger et al. 2006). This is because despite their similarity, the two measures communicate slightly different propositions: while $m_{\text {eff }}$ specializes in the issue of fragmentation and hence analyses the connectivity of existing habitats, the UZVR-approach examines the landscape from a broader perspective and takes into account the total area, assuming that the entire area would potentially be available as natural or semi-natural habitat.

Both measures face an identical problem when it comes to the yearly comparability of the aggregated final result: the calculation of UZVRs and of $m_{\text {eff }}$ for large countries or territories bears the risk that a result can augment between two points in time and thus indicate an improvement, while the issue of fragmentation for the landscape as a whole may have aggravated. This would for instance be the case when an already large unfragmented area doubles in size, while three smaller areas of particular ecological value for their regions are lost. This problem intensifies for the calculation and communication of $m_{\text {eff }}$, because as stated above, the metric usually considers habitats only and hence assumes $A_{\text {total }}$ to be the sum of all patches. This means that the result of $m_{\text {eff }}$ can increase, and hence improve, between two points in time, when an entire small patch is lost (assume that for instance in the year 2050 there are three patches of the sizes $1 \mathrm{~km}^{2}, 1 \mathrm{~km}^{2}$, and $2 \mathrm{~km}^{2}$ producing a $\mathrm{m}_{\text {eff }}$-value of 1.5 , and that in the year 2055 one $1 \mathrm{~km}^{2}$-patch is lost, which leads to the value of $m_{\text {eff }}$ being 1.67 ).

Therefore, any communication of the effective mesh size in particular has to also include information on the development of the area available as habitat, because connectivity results alone might improve while the overall availability and quality of habitat 
deteriorates. Apart from the possibility to supply the necessary data on land use, this problem could also be overcome by considering the entire landmass as total area in $m_{\text {eff. }}$ An increase in area not suitable as habitat would lead to a lower result of the $m_{\text {eff }}$ formula. In this case however, it would not be clear if a lower value was caused by an increase in fragmentation or an increase in build-up area. Overall, it is concluded that $m_{\text {eff }}$ should focus on its strength, which is measuring fragmentation, and therefore concern as "total area" only the sum of patches. This is viewed tolerable because researchers and decision-makers working in the field can be assumed to know that for a comprehensive and thorough environmental analysis of an area, several important characteristics of a landscape need to be considered. While connectivity is one significant aspect, qualities and sizes of ecosystems and habitats also are substantial characteristics.

One limitation of the $\mathrm{m}_{\text {eff }}$-method can be the application of a cutting-out procedure, which means that administrative borders are assumed as patch-boundaries. This is not normally the case, unless territories are separated by natural elements like oceans, very large rivers, or very high mountains. The alternative of leaving out boundary areas bears the risk that the share of the remaining total area is far below the actual area under investigation and that the result is not representative. In 2007, Moser et al. developed an alternative approach, which is called cross-boundary connections procedure. In the modified $m_{\text {eff }}$-approach, an administrative boundary is not considered to fragment patches, only physical barriers are. In order to then calculate $m_{\text {eff }}$, factual sizes of the boundary patches, that is including their extension into areas outside the region under discussion, need to be known. The idea of the $m_{\text {eff-approach is maintained }}$ as in the extended formula the probability of a randomly chosen first point to lie within the area of the reporting unit is multiplied with the probability that a second randomly chosen point lies in the complete area of that patch, be it in- or outside the original reporting unit's border. When summing up, a boundary patch contributes to each reporting unit according to its share within the unit. Moser et al. describe this characteristic as "area-proportionately additive" (Moser et al. 2007).

While degrees of fragmentation are determined in more detail by the $\mathrm{m}_{\text {eff }}$-metric and hence are of value to society and to landscape and infrastructure planners, for an individual road user, identified locations of undissected low-traffic areas can be a starting point to assess the fragmenting impact of a route. However, the information remains rather vague. Using it for quantification seems too complicated and not resilient enough to work with in management and mitigation of environmental impacts. 


\subsection{Summary and Conclusion}

The analysis of the demands of environmental management systems yields that the environmental impacts of rail, road, water, and air transport receive limited emphasis. Secondly, when ecological impacts of transport are referred to, both in management systems and in corporate reports the focus is on energy demand and emissions, and here particularly on greenhouse gas emissions. Further impacts are rarely referred to.

The survey of environmental management tools used by public authorities in section 3.2 proved to be of limited use for corporate and organizational entities as well as for the development of road user-specific indicators. Participating in an enforcement of a SEA, EIA, or an Ecological Risk Analysis may be helpful to learn more about systemic as well as about site-specific impacts of the construction and operation of plants and transport infrastructure. The DPSIR-model may allow viewing impacts at a broader perspective, where individual action is not limited to physical impact, but has the potential to affect other players. While the precautionary principle is a general guideline not limited to the assessments of public authorities, it was chosen to be referred to at this point because it is regarded to be of importance when impacts that potentially affect biodiversity are concerned, as these are usually characterized by significant human uncertainty of high ecological complexity. Moreover, particularly in the realm of indicator development it seems relevant to maintain a critical and precautionary mindset and not solely to rely on seemingly hard-fact indicators and definite thresholds when it comes to environmental impacts. The results and the functioning of the landscape-level indicators $\mathrm{UZVR}_{100}$ and $\mathrm{m}_{\text {eff }}$ that were presented in section 3.3 give an overview of the character and the conditions of the impacts in industrialized countries, but they are found incapable to contribute to the formation of indicators of a single user's impact or to immediate corporate management of the impacts.

Despite the continually high relevance of measuring and mitigating greenhouse gas emissions, the existence of other environmental impacts should not be neglected. Considering the depth of life cycle assessment and the variety of environmental impacts accounted for in eco-balancing, it becomes clear that further impacts are not really neglected. However, environmental management standards and guidelines still are incomprehensive. Two reasons for this deficit come to mind: firstly, some of the impacts like neophytes or fragmentation are relatively new phenomena, at least at current aggregate levels of impact. The latter condition of significantly augmented aggregate levels of impact also applies to land use and noise originating from road use. This explains why indicators for these impacts are either missing, or in the case of land use, seem to have room for improvement. Secondly, while the topic of greenhouse gas emissions was kind of a bottle opener also to become aware of the occurring loss of 
biodiversity, it at the same time seems to absorb capacities and to allow society and corporate producers of goods and services to constrain responsibility and awareness to greenhouse gas emissions. Indications that this slowly is changing for instance are the increasing levels of consideration of biodiversity in environmental management tools. Next, related but less easily discernable impacts like invasive alien species, fragmentation, or indirect land use for instance from agricultural fertilizer or traffic noise need to be considered. Due to their underrepresentation in environmental management and due to their high relevance in disturbing and threatening ecosystems and species, in the remainder of this work the impacts of degraded area and fragmentation will be focused upon. The targets are firstly, to better understand the impacts, which is the goal of the analysis carried out in the following chapter, and secondly, to develop quantifiable indicators that can facilitate their inclusion into environmental management (Chapter 5). 



\section{$4 \quad$ Selected Ecological Impacts of Roads and Traffic}

While carbon dioxide relevant emissions are the environmental impact of traffic most acknowledged and focused on at the political, societal, and also at the corporate level, a number of other environmental impacts exist. In this chapter, the three effects of land use, fragmentation, and road mortality are described. Referred to first in section 4.1 is the impact of road mortality. This is not so much because it is the commonly first perceived impact, but because it is interrelated with the other two effects of habitat degradation and fragmentation (Oggier et al. 2007). For instance, if road mortality is high, this can also constitute a barrier effect because too few attempted crossings are successful. It can also be a problematic sign if road mortality is very low, either because fencing keeps animals from entering and passing a road and hence increases the barrier effect, or because animals are deterred by the effects of traffic to such a degree that they do not approach a road, which is likely to imply that a large area of habitat is degraded or lost. For being more intuitive, next, in section 4.2 the effect of habitat loss is turned to before in section 4.3 the effect that is most difficult to measure and fully to understand, which is fragmentation, is examined. Further impacts that are not referred to in more detail here are the entire life cycle impacts of a road, a vehicle, and fuel. Effects then originate from polluting activities like $\mathrm{CO}_{2}$-intensive cement production or toxic copper extraction and the spatial demands from accessing lowly-disturbed areas for fuel production like the Arctic. In addition, moving vehicles can function as carriers for invasive alien species. Another type of impact are substance discharges like greenhouse gas or nitrogen emissions, which can affect ecosystems at much wider scales than can the area defined as degraded habitat referred to in section 4.2. At this point in time special emphasis resides with the emission of carbon dioxide equivalents. Greenhouse gas emissions from road transport accounted for $9.9 \%$ of the global emissions in 2000 (Baumert et al. 2005). For Germany, $\mathrm{CO}_{2}$-equivalent emissions of the entire transport sector in 2012 accounted for $16.72 \%$ of gross national emissions (UBA 2013). Furthermore, roads often facilitate erosion, sedimentation, and they may produce changes in hydrologic regimes (for a more complete description of effects see Friedrich \& Geldermann 2013a (Article I) and Forman \& Sperling 2011). While effects of roads and traffic that are perceived positively to influence the diversity of ecosystems also exist, a complete review of the empirical literature found the total ratio of harmful and beneficial impacts to be about 5 to 1 , thus proving a significant one-sidedness (Fahrig \& Rytwinsky 2009). The focus here is on land use and fragmentation because besides greenhouse gas emissions they are viewed to be the next most harmful impacts to ecosystemic, genetic, and speciose diversity (cp. Figure 2.1), and because they are still missing in environmental management, eco-balancing, standardization, and life cycle assessment tools. 


\subsection{Road Mortality}

Of the three major impacts dealt with in road ecology research, which are road mortality, loss and degradation of habitat, and fragmentation, animal casualties are the most devastating impact at the individual level (Bertiller et al. 2007, EEA 2011, Roedenbeck et al. 2007). Apart from the animals that are specifically attracted by a road, animals collide with vehicles because of their natural needs for movement either within habitats or through the farther landscape. It is distinguished between animal movements within a population and those exceeding a population's habitat. Movements within populations take place in order to forage for food, to find places to rest or to sleep, to reach mating locations, or to change between seasonal habitats (cp. Taylor et al. 1993, Oggier 2007). Since these movements in general are of shorter distances they are also called home range or trivial range movements. Relatively larger movements between seasonal habitats are referred to as migration range movements (Reck et al. 2005). Movements in which animals exit a population can occur because of seeking new habitats and partners for reproduction or in order to disseminate and to recolonize uninhabited areas. These movements usually imply covering longer distances and are referred to as dispersal range movements (Bisonette \& Adair 2007). Hence, there exist a variety of motivations for animals to move through the landscape.

In landscapes with dense infrastructure networks animals often encounter roads or railways. When animals are not sufficiently aware of the awaiting danger, collisions can be a lethal consequence. Animal mortality has been attempted significantly to be reduced by mitigation measures like wildlife fences, separation barriers between lanes of opposing directions, traffic volume and/ or speed limits, wildlife reflectors, road-verge management, warning signs for drivers, and wildlife crossing structures (van der Grift et al. 2013). While more experiments on most measures and particularly on their interplay in order to function complementarily are needed, casualties particularly in the large road network of minor roads can hardly be fully avoided before putting road infrastructure underground.

Despite various efforts, the quantity of casualties is still significant. While deer, foxes, hedgehogs, or rabbits are particularly threatened at an individual level, road mortality in Germany in the past even was the number one cause of death for stone marten, viper, or wildcat (Herrmann et al. 2007). Mainly for the reason of road mortality, the latter two animals in Germany are listed in the threatened species list (BfN 2009). In North America, the number of estimated animal-vehicle collisions is 1-2 million (references in Huijser et al. 2009). In Austria, in 1998 a deer was more probable to die from traffic collision than to die a natural death (Oggier et al. 2007). 
Eminently concerned to die from collision are slow-moving species like amphibians, hedgehogs, or vipers, animals that do not behave or react 'intelligently' since they cross at an angle or spend more time in the road than necessary, species with diurnal movement patterns, and species with larger spatial demands that have more extensive movement ranges that encounter roads more frequently like wild cat, deer, or lynx (Hels \& Buchwald 2001). Further findings suggest that larger rather than smaller mammals are subject to collisions because smaller ones perceive a road as a barrier more quickly and hence are deterred more often (cp. Bertiller et al. 2007). While the degree of vulnerability also depends on differing behavioral reactions of individual species and sometimes individuals, mainly for their diurnal movement pattern and the low velocity of movement, studies found anuran and amphibian species to belong to the group of animals particularly threatened by road mortality (Eigenbrod et al. 2009).

At the population level, road mortality is a serious threat to the existence of populations that have low reproductive rates, such as wildcats or to a minor degree to bats (Herrmann et al. 2007, Kerth \& Melber 2008). If a mortality rate is higher than a birth rate and the number of remaining individuals falls below the value of the minimum viable population size (MVP), a population collapses. Gibbs and Shriver (2002) found that as little as $2-3 \%$ of additive annual mortality is likely to be more than most turtle species can absorb to still maintain positive population growth rates. In addition, species are particularly vulnerable also at the population level, if they have smaller populations and are rather rare species (cp. Spielman et al. 2004). A population is also particularly threatened by road mortality, if it is regulated by density-independent mechanisms like climate variability, because then road mortality is an additive and hence important factor, than when it is regulated by density dependent factors (mainly intraspecific competition among larvae) (Hels \& Buchwald 2001).

Besides the variables inherent to the animal, characteristics of the vehicles and the road also influence the level of risk of an individual. While road width and vehicle speed are contributing factors, various studies identified traffic volume as the clearly dominating factor for attempted or successful crossings (Jaeger et al. 2005b, Charry \& Jones 2009). While in general the number of casualties correlates positively with the number of vehicles per day, for some species there exist thresholds when the frequency of passing vehicles and resulting noise frighten animals to a degree where a road becomes a complete barrier. Avoidance then reduces the number of casualties. Studies specifically investigating traffic volumes suggest that roadkill rates increase as traffic increases up to a level of about 3,000 vehicles per day. For traffic levels between 3,000 and 10,000 vehicles the rate remains high or may begin to drop and it on average drops further for levels above 10,000 vehicles as the intensity of vehicles and noise significantly deters animals from crossings (Charry \& Jones 2009, Forman \& Sperling 
2011). This is positive only in part because it means that the effect of the road as a barrier then is substantial.

While average road kill rates decrease with daily traffic volume and hence are lower on minor roads, the total effect of minor roads is assumed to still be significant due their high quantity of occurrence in the landscape (Taylor \& Goldingay 2010). Overall, population size, species occurrence, velocity of movement, and diurnal movement patters as well as the number and characteristics of vehicles, are the most important factors influencing species' vulnerability to road mortality (Hels \& Buchwald 2001; Riley at al. 2006). Before moving to fragmentation, habitat loss and degradation are turned to.

\subsection{Loss and Degradation of Habitat}

Habitat loss and degradation are grouped together as the second negative impact caused by road infrastructure and traffic. The soil and habitat functions of natural area can either be lost completely where surfaces are sealed, or they can be lost partially in the area adjacent to a road where substances or disturbances take effect.

The sealing of a formerly natural area is problematic not only because a mammal's or plant's habitat is lost, but because sealed area also loses its service capacities like soil fertility, erosion protection, oxygen production, water purification, or waste treatment. For the road subgrade, the foundation, and the surface, soil is removed and replaced by gravel, bitumen, and concrete. The thickness of these layers is between 0.5 and 0.6 meters in total, depending on the type of road (Maibach et al. 1999). For Germany, area sealed by transport infrastructure in 2012 accounted for $5.05 \%$ of the total land mass, that is $18,032 \mathrm{~km}^{2}$ of $357,690 \mathrm{~km}^{2}$ (Federal Statistical Office $2013 \mathrm{~b}$ ). 5 Only considering the categories commonly related to road bound transport, which are 'road', 'path', and 'place' (Keys 510-539), 15,708 km² or 4.40\% remain. While this distinction excludes areas of all types of rail, aircraft, and water shipping, it does not differentiate between motorized and non-motorized means of movement like walking or cycling, or between places associated with traffic and places of uses other than travel or transport. The analysis at hand considers faster and farther movements; and thus motorized vehicles. Hence, when excluding several types of uses like sidewalks, bikeways, or marketplaces, and when further omitting concomitant areas of roads (Key 591), the value

5 The yearly publication "Surface area accorting to actual usage" indicates all surface uses in Germany. Keys of type of use (Nutzungsartenschlüssel) are employed to differentiate between the usages. Area for settlements and transport has the keys 500-595. This classification will slightly change in 2015 when automatic land registries of the federal states as data souces will be substituted for the official land survey register information system (ALKIS). 
of $15,825 \mathrm{~km}^{2}$ decreases. In Germany, federal states are not required to report as detailed as one digit units, which contain the respective information. One federal state that reports as detailed as one digit units is Rhineland-Palatinate. At the end of 2012, infrastructure that specifically served motorized road-bound vehicles accounted for $97.52 \%$ of the overall 'road, path, place'-category. As Rhineland-Palatinate is neither a predominantly territorial nor a predominantly built-up state, the inaccuracy from extrapolating the percentage to all of Germany is viewed acceptable. Hence the final value of sealed area that serves motorized road-bound traffic is about $15,318 \mathrm{~km}^{2}$ or $4.29 \%$. Relating this number to a non-vehicle differentiated total national motor vehicle mileage of 2008 of 690.1 billion kilometers, the share of sealed area of $1 \mathrm{~km}$ travelled is 222 $\mathrm{cm}^{2}$ or $1 \mathrm{~m}^{2}$ for every $45 \mathrm{~km}$ travelled (Elsner 2010). This very roughly calculated number will be discussed further in section 5.3, when it is used to test the results of the indicator of sealed area for an individual user obtained from the approach developed in section 5.1.1.

While in the area adjacent to a road, the functions of the soil are mostly maintained, the suitability of that area as habitat for flora at low distances and for fauna at longer distances can either be significantly impeded or lost. Adjacent area often is affected by additional artificial construction, like embankments, and predominantly by diffusing effects, like noise or substance emissions. In literature the zone over which significant ecological effects extend outward from a road, is called 'road-effect zone' (Forman \& Deblinger 2000). Besides material emissions from vehicles, such as nitrogen oxide, particulate matter, ozone, carbon monoxide, carbon dioxide, sulfur dioxide, nonmethane hydrocarbons, rubber and litter, and from the road, such as salt and dust, intangible emissions like vibration, heat, noise, and light, occur (Friedrich \& Geldermann 2013b). Furthermore, the road and the physical modification of the landscape can produce changes in local climate, as winds, temperature, and moisture can change. Also hydrologic systems are altered because roads can block water flows, increase the risk of flooding, contribute to erosion, and facilitate sedimentation into waterbodys (Forman et al. 2003).

The longer term effects from fragmentation, that occur at a population or even ecosystem level, in literature so far are not explicitly considered to contribute to forming the road-effect zone. Despite the impacts of fragmentation not being appropriately represented in the formula to be developed for the quantification of the expansion of roadeffect zones in section 5.1.2, at least to some degree the indicator can be assumed to account for it. It is laborious and costly to measure and hence difficult to estimate the spatial extension of impacts from fragmentation. The meaning of the effect only is beginning to be introduced to and comprehended by society. This has respective negative consequences on funding, meaning that little research at the genetic level has been 
done. The example that assuming a road-effect zone to extend 20 kilometers in a certain direction because the wild cat no longer inhabits the area, shows the partial and weighty incompatibility to account for the effects of habitat degradation and fragmentation in the same spatial indicator. While anurans that lose access to a forest suffer habitat degradation from a road barrier, the symptom of coyote populations showing signs of genetic depletion at 20 kilometers of distance from a road is not suitable to declare the entire area as significantly ecologically degraded. Concluding, genetic effects from fragmentation should only be considered to contribute to the degradation of an area, which should and is likely to be degraded predominantly by other impacts. Even foregoing a utilitarian assessment, the wide expanding impacts are not direct enough and apply to too few plant and animal species as for entire regions to be significantly degraded in their capacity to contain functioning and species-rich ecosystems. However, this assessment remains problematic, because reduced genetic variability in fact does degrade a habitat. As it cannot fully be considered by the concepts of significantly degraded habitat or the road-effect zone, it is important to be represented otherwise. The indicators to be built in this work do not express the spatial dimension of fragmentation, but assign abstract values to the assumed overall severity of the effect produced by a single road and its users. For the widely-expanding spatial impacts of fragmentation an impact and a terminology different to 'road-effect zone', such as 'area of wider impact' or 'less direct impact zone' could be employed.

To date, studies found that outward effects of roadways with significant impact extend from about 15 to 200 meters for material emissions, which can repress the germination of plants and can change the competitive relations and hence plant composition, to 2,000 or 3,000 meters for noise, affecting sensitive species like moose or breedingbirds (Reck \& Kaule 1993, Kaseloo 2006, Shanley \& Pyare 2011, Benítez-López et al. 2010). Other investigated animal species include salamanders, which were found to be affected by forest roads for a distance of up to 35 meters (Semlitsch et al. 2007), and tortoise and anuran species that were affected for up to 400 and up to 1,000 meters respectively (Boarman \& Sazaki 2006, Eigenbrod et al. 2009).

Noise is the most spatially extensive direct impact. Already a passenger car can project a noise level of $20 \mathrm{~dB}(\mathrm{~A})$ at a distance of one kilometer from a road. This sound pressure level exceeds the mean natural level of low frequency sound in most environments (Barber et al. 2010). Noise can inhibit intentional communication, such as song used for mate attraction, messages of territorial defense, alarm calls, and socializing contact calls (Slabbekoorn \& Ripmeester 2008). In addition, it can inhibit the perception of adventitious sounds used to detect and localize prey, predators, or other dangers like fire (Blickley \& Patricelli 2010, Grafe et al. 2002). Noise is assumed to provoke the same deleterious physiological responses in animals as in humans, including hearing 
loss, elevated stress hormone levels, and hypertension at exposure levels of 55-60 $\mathrm{dB}(\mathrm{A})$ or higher (Barber et al. 2010, Babisch 2003, Jarup et al. 2008).

In order to communicate and hear successfully, signals must be detectable in background noise. The signal-to-noise ratio (SNR) and the masked auditory detection threshold of the receiver determine the detectability of a signal (Patricelli \& Blickley 2006). Masking is the effect, when noise inhibits the perception of sound. The amount of masking is the difference between a masked auditory detection threshold, which is the threshold at which a signal can be perceived when a specific level of masking noise is present, and an unmasked threshold, which is the quietest level at which a signal can be perceived without any masking noise present. Most likely because effectively transmitted vocalizations favor natural selection, many species have evolved signals that maximize the habitat-specific SNR (Brumm \& Slabbekoorn 2005). Shifts in behavior and vocalization that can reduce masking by background noise can be increased sound level, increased duration of call syllables, altering of the duration and time of day singing, and, most observed, shifts in frequency or spectral energy away from the spectral energy of the noise (Brumm et al. 2004, Patricelli \& Blickley 2006, Helldin et al. 2013). Such adjustments testify an ability of "vocal plasticity" that was found for some bird and primate, and also for cetacean species (Wood \& Yezerinac 2006, Barber at al. 2010). However, in addition to having observed species that showed no such efforts of adaption, also for the ones changing vocalization it is unknown whether they are hence thriving as a consequence of undertaken efforts of adaption or whether shifts are symptoms of communication breakdown (Lengagne 2008, Slabbekoorn \& Ripmeester 2008, Patricelli \& Blickley 2006). Strongest evidence on the harmful impact of traffic noise stems from studies that found reduced reproductive success and decreased population densities near sources of noise (Reijnen et al. 1997, Patricelli \& Blickley 2006, Dooling \& Popper 2007, Lengagne 2008, Halfwerk et al. 2011).

Lighting can impact animal and plant species because together with temperature and humidity it is one of the most important daily and seasonal zeitgeber. Most organisms have evolved molecular circadian clocks that are controlled by natural day-night cycles. These clocks play key roles in metabolism and health, growth, and behavior (Dunlap 1999). A substantial proportion of global biodiversity is nocturnal, namely $30 \%$ of all vertebrates and more than $60 \%$ of all invertebrates (Hölker et al. 2010). Light pollution not only threatens biodiversity by the means of changed night habits and processes, in addition, it can misguide animals or lead them to locations of danger (Rich \& Longcore 2006, Friedrich \& Geldermann 2013b). The percentage of land area that is affected by light pollution varies by the levels of artificial brightness defined to be polluting (Gallaway et al. 2010). Cinzano et al. (2001) found that while in 1996/1997 85.3\% of the EU's land mass and $61.8 \%$ of the US' land mass had night skies where artificial 
light added at least $11 \%$ to the natural brightness of the sky at zenith, for higher levels of light pollution this percentage drops to only $0.1 \%$ of the EU's and $0.6 \%$ of the US' land mass when artificial brightness higher than 27 times the natural levels was considered. While no studies on the expansion of light from roads exist, nighttime traffic plays a major role in dispersing light pollution, which used to be concentrated in and limited to cities and industrial sites.

Going back to considering all habitat relevant impacts of road traffic, a non-species specific study found that $33.68 \%$ of the significant impacts lie within the first 100 meters, while only $8.09 \%$ of the impacts occur further than 500 meters away from a road (Biglin \& Dupigny-Giroux 2006). It is not possible to define an average width of roadeffect zones because its extension predominantly depends on traffic volume and is also influenced by the topography of the landscape (Shanley \& Pyare 2011, Charry \& Jones 2009). For a highway with about 50,000 vehicles per day, Forman and Deblinger (2000) estimated the extension of significantly affected habitat to average approximately 600 meters. Smaller values apply for less travelled or secondary roads. With regard to the presence of effects at the landscape level, expanding road effects result to be a relevant ecological problem. In 2000, Forman calculated that while roads in the USA cover $1 \%$ of the land, they essentially affect $20 \%$ of the total land mass. It is unclear whether a likewise correlation between sealed and affected area applies to Germany. There, in 2012 the area covered by motorized road-bound infrastructure was $4.29 \%$, which assuming a similar correlation would yield a percentage of affected area far beyond $50 \%$ (Federal Statistical Office 2013b; calculations extended by the author as indicated above). However, as the road grid in Germany on average is more dense than is its American counterpart and as there are only six undissected-areas larger 500 $\mathrm{km}^{2}$ that make for only $1 \%$ of the total land mass, there is reason to assume that such a surprisingly high number is correct (BfN 2013). It is also possible that this approximation still under- rather than overestimates the impact of roads across the German landscape. As a general assessment, Jordaan et al. (2009, p. 12) constitute that while "edge effects are not the same as direct habitat removal, they will likely result in reduced habitat effectiveness".

\subsection{Habitat Fragmentation}

Several studies conclude that besides the destruction of habitats, also their fragmentation represents one of the greatest threats to biological diversity world-wide (Forman 1995, Jaeger 2000, luell et al. 2003, MA, 2005, Lindenmayer \& Fischer 2006, Smith \& Smith 2009, CBD 2010a). While the impact has different peculiarities for instance for southern hemispheric native forest roads or for highways in densely settled and con- 
nected societies, it is of significance in most landscape structures around the globe. By definition, landscape fragmentation is the result of transforming large habitat patches into smaller more isolated fragments of habitat (EEA 2011). Despite being particularly apt to produce fragmentation, man-made roads are not the only element to act dissecting. Fragmenting elements of a landscape can be natural features, such as rivers or mountains, and anthropocentric features, such as intensively used agricultural areas, industry and settlements, and, most impacting, the different types of linear transport infrastructure (Mader 1984). Concerning roads, some researchers regard the resulting phenomenon of fragmentation to consist of two slightly different effects: the subdivision of populations, referring to the division of meta-populations into smaller subpopulations, and the barrier effect, meaning that animals, but also plants, are hindered to move freely within a landscape (Bertiller et al. 2007, Jaeger et al. 2005b). As a dissection of populations can only take effect if a road represents a barrier, in this work both impacts are subsumed under the barrier effect. A road can represent a barrier in three principal ways:

1) Animals can be deterred to approach a road because of noise, light or changes in habitat. In addition, when they do come up to a road, they can be deterred to attempt to cross it, either because of physical obstacles like fences, median barriers, or even the road surface, which may display uninviting conditions for being dry or for being without shelter, or because of the presence of fast moving vehicles, which increase levels of noise and stress. This applies for life on the ground, in the air, and in streams.

2) Animals that attempt to cross a road can die in a road. This explains why road mortality was referred to above. Despite being a discrete impact, unsuccessful crossings significantly contribute to the barrier effect. Animals predominantly die because of collision with vehicles, but they can also get killed by attacks of waiting of predators.

3) Spores, insects and other life forms that do not actively cross a road but are carried over by host animals can no longer disperse, if their host is susceptible to one of the before mentioned aspects that keep her or him from crossing a road.

The barrier effect is problematic because it impedes or blocks either an individual animal's or an entire population's access to resources and to mates (Bertiller et al. 2007). As indicated above, it can be differentiated between movements within a population and movements between populations. 'Home range' movements that take place within a population are more likely to be shorter daily displacements for instance to reach food, to sleep, to mate, or to change between summering and wintering habitats. In the latter two cases the displacement is likely to take place during a period longer than one day, but is still likely to happen within a population. 
Despite the significance of a road to represent a barrier to presently living individuals or populations, a larger threat on the abundance, fitness, and eventually the persistence of future populations comes from the potential lack of genetic interchange within and between meta-populations, and even between individuals from much farther away territories that can be triggered by the barrier effect of a road. The up-keeping of functional ecological relations between species and ecosystems both over closer and farther distances is viewed as a major prerequisite for the conservation and flourishing of biological diversity (Reck et al 2005). This applies in particular to the intensively used landscapes of central Europe.

Movements that exceed the own population can be 'dispersion', when individuals undertake non-recurring, non-target oriented movements in order to find new mating grounds or partners, or 'dissemination', when individuals settle down in uninhabited areas (Oggier et al. 2007). All types of movements happen for reasons that are likely to be essential for the survival of individuals or the long term fitness of populations. Compared to other effects like road mortality or lost access to resources, the negative consequences from denied access to mates, which are inbreeding or decreased variability of genomes, will possibly become the most problematic impact of fragmentation. Reasons for this assessment are the ecological severity of the impact, its low visibility, and to date sparsely understood risks and patterns of extinction (Jaeger et al. 2005a). A number of studies exist that found a genetic subdivision in animals and plants due to fragmentation (Riley et al. 2006, Gerlach \& Musolf 2000, Fischer \& Matthies 1998). Keller et al. (2004) found that the risk of genetic differentiation and extinction due to the barrier effect is significantly higher in species with low dispersal abilities as for instance in flightless ground beetles or some amphibians and reptiles. Also particularly likely to be adversely affected are specialist species that require niche habitats (Fahrig 2003) and mammals with large habitat demands, such as lynx, needing at least $100 \mathrm{~km}^{2}$ of habitat, or wildcat needing up to $30 \mathrm{~km}^{2}$ (Yahner 1988, Herrmann et al. 2007). The time lag between the occurrence of an impact and the full ecological consequences in a landscape can take decades. This phenomenon is referred to as "extinction debt" (Tilman et al. 1994). That effects on genetic variability are both difficult and costly to examine is a problem for research and particularly for the societal perception of the impact. More information needs to be generated by applying molecular approaches that investigate genetic consequences to a higher degree (Balkenhol \& Waits 2009). They are particularly needed to investigate the effectiveness of wildlife crossing structures at the 
population level, as their construction is the central feature of most countries' efforts of defragmentation (Corlatti et al. 2009, van der Grift et al. 2013). 6

Another difficult issue is the question, at which traffic volume a road turns into being a significant barrier. While the overall correlation between traffic intensity and a road displaying a barrier is positive, the identification of exact thresholds is much more difficult because they vary between species and depend on the level of ambition of the goal, i.e. whether basic connectivity or near full permeability shall be reached (Charry \& Jones 2009, Taylor \& Goldingay 2010). Connectivity and permeability describe similar phenomena, but they are not identical. Permeability can be described as the highest possible order of plant and animal movement, meaning that animals can move freely without encountering dangers or barriers in almost any location. Connectivity refers to the possibility of movement at the next lower magnitude. It implies that for some individuals of most species it is possible to reach adjacent habitats, but that this is not as easy as in a permeable state because locations safely to reach another place are much fewer. Connectivity is important to enable dispersal range movements in order to maintain variable gene pools and to re-inhabit singular landscapes. It aims to meet the minimum requirements for movement of functioning and stable ecosystems. Connectivity does not meet the demands of all individuals and likely neither of all species. Both terms express the possibility of animal movement within a landscape, where connectivity is the least necessary condition to connect habitats and where permeability would be the ideal situation of nonhazardous movements in most places.

More recently, humanity took a path of development that today results in a desirability and conduct of mobility intensive lifestyles for the majority of people in industrialized and industrializing countries. Although the demands of some individual people and countries may willingly or unwillingly decrease in the future, for instance even for Germany, in total a further increase in traffic volumes is expected. A slight relativization of this development is made by the annotation that further increase is expected "at least for the nearer future" (BMVBS 2014, p. 1). Complete permeability of the landscape then would be an ecological target that is impossible to be reached. Yet, it is assumed that for a stable functioning of ecosystems, a high degree of connectivity in the landscape might be sufficient. Certainly, the goal of establishing connectivity needs to be sidelined by other measures like fencing and signaling in order to reduce rates of road mortality. The objective of spatial planners and of infrastructure users could be to guarantee permeability at smaller habit levels and to warrant connectivity at the larger landscape level. With regard to local and regional habitats, this requires a certain percent-

6 For a discussion of strategies of defragmentation see Friedrich \& Geldermann 2013b. 
age of a country's total area to be largely undissected and undisturbed. To monitor this goal, in Germany the method of non-dissected low traffic areas is used as one of 19 indicators of the national strategy on biodiversity.

Going back to the question, at which daily traffic volume a road would represent a barrier, Charry \& Jones (2009) identified a traffic intensity of 10,000 vehicles per day as a near complete barrier for most species. Moreover, they recommend avoiding to increase daily traffic volume to more than 3,000 vehicles per day in locations where roads are not wanted to have a significantly negative environmental impact either due to road mortality or the barrier effect. While Forman \& Sperling (2011) adopt this number of 3,000 vehicles per day as a threshold of substantial ecological impact, a study of the German Federal Ministry for the Environment, Nature Conservation and Nuclear Safety (BMU 2010) assumed a value of 1,000 vehicles per 24 hours as a threshold of significantly harmful impact. This accords to findings from Alexander et al. (2005) who found that in the Canadian Rocky Mountains the movement for carnivores was significantly impaired already at traffic volumes of 300 to 500 vehicles per day and that for ungulates the range of traffic volume of respectively impairing impact was between 500 and 5,000 vehicles. A study on salamanders in Appalachian Forests found that even a decommissioned gravel road can represent a barrier for some amphibian species (Semlitsch et al. 2007). This range of findings shows, how much the definition of a road with a certain traffic volume as a barrier, depends on the species of concern and the underlying objectives.

Some researchers argue that the most impacting effects from fragmentation on ecosystems come from the impeded movement of larger mammals. Apart from their own fate, they are assumed to be creators of habitats, because their movements and the impact of their hooves physically alter landscapes, and because they have a vector function as they move around smaller insects and plant seeds that they carry in their fur (Eversheim et al. 2009). Studies indicate that the barrier effect of a road for this group of animals lies above the more conservative value of 1,000 vehicles per day, namely rather around 3,000 to 5,000 vehicles per day as assumed by Charry \& Jones (2009) and Forman \& Sperling (2011).

In order to describe the often separately indicated second consequence of landscape fragmentation, that is the subdivision of populations, it is helpful to distinguish between two biological group concepts of life: the population and the meta-population. A population is a group of individuals that lives in the same habitat and that reproduces amongst each other. If a few habitats are scatteredly located within a greater landscape, individuals will only occasionally disperse and connect with other populations. This system, where dynamic and discrete populations interact only every so often, is called a meta- 
population. From fragmentation, either a subdivision of a meta-population, or a subdivision of a smaller population itself can happen. The four negative effects that newly generated subpopulations are then facing are the same in either of the two cases, but they are much more severe if a population itself is divided. One effect is the edge effect, which means that the edging and less favorable area increases while the core habitat decreases. Secondly, demographic instability increases. This means that the gender ratio or the ratio of individuals at reproductive age opposed to those not at reproductive age might be less favorable. Thirdly, changing environmental factors are a much greater thread to concentrated subpopulations. Fourthly, genetic variability in a diminished population always declines (Oggier et al. 2007). Again, in this work the barrier effect and the effect of subdivision are subsumed under the barrier effect alone because the effects in the case of subdivision require a barrier and often apply to any barrier. Overall, the subdivision of a population is viewed to be a type of barrier effect that is particularly harmful.

\subsection{Summary and Conclusion}

In this chapter the three impacts of road mortality, habitat loss and degradation, and fragmentation were introduced. It is evident that significant ecological impacts of the transportation process exist that extend beyond carbon dioxide emissions. The negative effects referred to here are particularly valid for road transport but to a lower degree also apply to other transport infrastructure types and vehicles like rail, inland navigation, aviation, and also to power lines.

While mortality and habitat loss due to road traffic usually threaten individuals and populations, they can represent as severe impacts as to endanger entire species that are particularly susceptible to them. Yet, in total, the fragmenting impact of the barrier effect seems most dangerous because it has the potential to structurally weaken species' genetics. While recolonization of habitats and species' abundance in certain locations immediately are impeded by barriers, reduced genetic variability develops subtlety and often becomes evident only after a long time. It can show in reduced genetic variability, which can affect healthy reproduction and which can decrease populations' abilities to adapt to external events like single weather extremes or long term changes in climatic conditions. Thus, impacts from fragmentation that derogate the long term fitness of populations and species are viewed to be particularly dangerous, also because they can show with great delay and are difficult, if not impossible, to reverse.

It is pointed out that the nature and severity of any of the impacts differs depending on the species and the respective behavioral responses that a species shows. While the 
stability of a population is particularly impaired if a species is struck by all three effects simultaneously, behavioral responses often tend to solve one of the threats only by aggravating the severity of another. Species that are put off by noise carry a lower probability to be run over, but face the tradeoff that the barrier effect of a road then has increased. Some amphibian species are less threatened to become casualties because they avoid dry and open spaces, which in turn also aggravates the barrier effect. As behavioral responses often determine which of the three effects has the greatest impact, it follows that particularly larger, non-traffic-dissected areas that cause neither of the problems, significantly improve the ecological quality of a region, at least as far as disturbances from transport infrastructure are concerned. The severity of ecological impact of a road correlates with traffic levels because they not only determine the risk of mortality in the road, but they are also sources of emissions like nitrogen oxide, particulate matter, light, and noise. These emissions degrade adjacent area and in deterring animals also contribute to the effect of fragmentation.

The negative consequences that the three effects of road mortality, habitat loss and degradation, and fragmentation have in common are reduced population size and higher risk of extinction (EEA 2011). Both symptoms mean a loss of biodiversity. This might pose a problem from a moral point of view, and also because the functioning of ecosystems depends on natural diversity (Belardi et al. 2011). Not every reduction in population size or extinction of a species leads to the collapse of an ecosystem. With regard to evolutionary time scales, they have shown to be very stable systems, if they are not disturbed by sudden external events. However, current extinction rates of species are 100 to 1,000 times higher than evolutionary rates and hence can be viewed as a sudden influence that tests ecosystem stability (Pimm et al. 1995, Pimm 2004). 


\section{$5 \quad$ Complementing Environmental Indicators}

Mainly due to the severity of the impacts and the resulting relevance to redress their misrepresentation in reporting, decision-making, and environmental management, the effects of land use and landscape fragmentation were chosen to be analyzed and prepared for easier inclusion in corporate efforts of environmental management. With regard to businesses, it shall briefly be recalled why a more complete set of indicators of ecological impacts from transport is needed. From a corporate perspective, it seems adjuvant to know about the impacts in order to make better informed decisions, independent of criteria hierarchy. Furthermore, having a more complete set of indicators is helpful because consumers urge companies have to improve their sustainability reporting and to operate more sustainably because their understanding of the meaning and desirability of sustainability is augmenting. For companies, it is easier to manage, that is to avoid and to mitigate impacts, if methodologies allow for their measurement and comparison. Despite agreeing with the notion that people in industrialized countries know enough about the environmental crisis, which means about its causes, the consequences, and the actions that lead to improvements of ecological conditions, in facilitating quantification of impact for a process, this work continues along the lines of contemplation rather than along those of action. Put in a drastic way, the demand of exact quantification can be viewed as an excuse for inaction. For instance is there scientific and political consensus on the unacceptability of human-induced climate change, but demands for more reliable forecasts or comparison between countries, sectors, or individuals lead to the postponement actions. While additional analysis allows for more effective and efficient attainment of goals, doubts on the necessity of further analysis are legitimate. The potential medium-term contribution of this work is seen in transferring seemingly landscape related impacts like land use and fragmentation to the individual provoking the impacts, both in production and consumption. While companies that have human resources of high quality in environmental management know about the impacts referred to here, quantification might increase the responsibility to consider the impacts more seriously, to refer to them in sustainability reporting, and to mitigate them. As indicated above, quantification also concerns the consumer, because awareness and comparability might allow her or him to contribute to avoid the impacts. Particularly in a lowly restricted free market economy, decisions in consumption play a central role in practicing one's democratic responsibility.

\subsection{Quantification of Land Use from Road Transport}

With regard to land use impacts from road transport, in this work it is differentiated between two types of land use impacts: first, the sealing of natural soil by the roadway 
and potential auxiliary construction, and second, the impacts of a road and its traffic that extend into the landscape. Due to a significant rise in complexity and decrease in precision when considering all roads of the entire road grid, and due to the assumption that road transport takes place mainly on larger roads as the mean haulage distance in Germany was $104 \mathrm{~km}$ in 2010, the analysis at hand focuses on transports on federal highways (German: Bundesstraßen) and freeways (German: Bundesautobahnen) (UBA 2012). While the formulae to be built can also consider the spatial ecological impacts of traffic on smaller roads like country roads (German: Landstaßen), district roads (German: Kreisstraßen), or municipal roads (German: Gemeindestraßen), the accuracy and type of impact here are less distinct. Thus, an amount of 1,000 vehicles per day is considered the threshold from where on the two indicators on land use yield accurate results. Concerning the share of use of smaller roads in an individual trip, results are particularly reliable for routes for which the share of distance travelled on roads with less than 1,000 vehicles per day does not exceed $10 \%$.

\subsubsection{Determination of the Share of Sealed Area of an Individual User}

The central question when determining the share of sealed area that an individual user is responsible for is on the surface sealed and hence on the width of a road. As no data on the individual width of roads and road segments is available, various approaches of assuming average road widths are possible. The two main approaches to differentiate between roads of unequal road-width are either by road category or by the quantity of lanes. The analysis at hand is tailored to longer transports, which mainly use larger roads like federal highways and freeways. Since sizes of roads within both categories vary significantly and since data on the number of lanes for all German roads of these two road types is available, both road type and the number of lanes are chosen as relevant factors to assume approximate road width. In the following section it is reasoned, which average values of lane width are assumed and which other parameters are considered to be relevant for the calculation of the share of sealed area per individual user.

\subsubsection{Measuring sealed area by the average width of road lanes}

The overall approach to determine the share of sealed area that an individual user is responsible for is to divide the sealed area by the number of users. In order to determine the sealed area, approximate road width needs to be known. Road width depends on the quantity of lanes and road type. As the quantity of lanes and the number of users change over the course of a route, a route is split into segments in which both parameters remain constant. Here, data from the publication 'Federal Roads of Germany - January 2012' and Germany's 'Manual Traffic-Census 2010' is used (BMVBS 2012a, 
Bast 2011a \& 20011b). A new segment begins whenever one of the two variables changes. Thus, in the first step, the area sealed within a section is calculated. In order to do so, information on the average width of the road and on the length of the section is needed. Information on the length of sections on freeways can be obtained from the '2006 Autobahn directory', which indicates the distance between all ingress ramps or exits (Bast 2007). Information on motorways constructed after 2006 as well as on federal highways or still smaller roads can most reliably be obtained using GIS- or route planning software. In order to calculate the average width of a road section using the above mentioned publication of the Federal Ministry of Transport, Building and Urban Development (BMVBS), the average width of sealed area per lane has to be determined. It is assumed to be 4.96 meters for federal highways and 7.45 meters for freeways. The ascertainment of these values is reasoned in the following paragraphs.

In order to estimate the area sealed by a road from the number of lanes, the assumed average lane width has to account for two types of areas related to the road: firstly, the roadway itself, and secondly, associated auxiliary construction, such as ramps or bypasses, junctions, and resting areas. The area covered strictly by a roadway is determined from guidelines for the design of roads. For Germany, a number of standard cross-sections per road category exist. The directives not only define the width of traffic lanes, but also the necessity and size of all additional elements that are part of a road. Lane width varies between 2.75 meters for district roads and 3.75 meters for large, high-traffic roads. Figure 5.1 shows most of the possible elements. These can include a median between lanes of opposing directions (here 3.50 meters), a marginal strip to prevent the breaking-off of roadway edge (here $0.75 \mathrm{~m}$ ), a curb designed for larger vehicles, for situations of emergency, or for conditions of high traffic (here $2.50 \mathrm{~m}$ ), and a shoulder to stabilize the entire road body and to install signposts, side rails, and noise barriers (here $1.50 \mathrm{~m}$ ). To account for the area consumed also by those elements, the average lane width exceeds the factual size of traffic lanes.

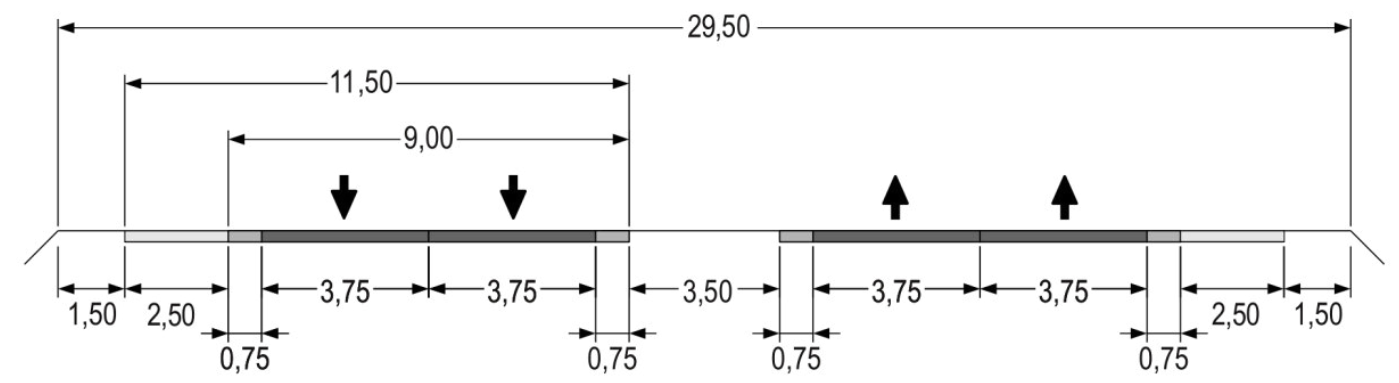

Figure 5.1: Standard cross-section 29.5 of a four lane highway as of RAS-Q and an indication of road elements; source: FGSV 1996. 
The directive used to calculate average lane widths that account for the entire construction is entitled 'Guidelines for the Design of Roads (RAS-Q)'. For the last 30 years this directive was valid in Germany. The guidelines of the directive apply to new roads only and not to all roads already present in the road grid. The name of each standard cross-section, e.g. 'Standard Cross-Section 26 - RQ 26' is identical with the width of the respective road. In 2008, a modified standard entitled 'Guidelines for the Design of Motorways (RAA)' was introduced and in 2013 it was followed by the 'Guidelines for the Design of Country-Roads (RAL)'. These two new standards render the formerly used RAS-Q standard obsolete. While a few standard cross-sections changed in size, e.g. RQ 35.5 now is RQ36, the changes are moderate and the present road grid is not concerned by the changes in regulation that apply only to to be built or to be modified roads. In fact, despite the RAS-Q standard was valid for new roads for a significant period of time, an unknown but estimated very large amount of roads do not yet adhere to the standard cross-sections as of RAS-Q. This applies not so much for freeways, but rather to smaller and less often upgraded or revised roads, including federal highways. This means that the currently best approximation of the average area sealed per lane remains as under RAS-Q (see Table 5.1). One problem restraining the accuracy of the average lane width per road type, which represents the entire construction of a road and not strictly traffic lanes, is that within each category there exist several standard cross-sections and that no data on their proportionate occurrence in the road grid is known. Hence, the average lane width per road type was yielded assuming an equal distribution of standard cross-sections. It can be seen that lane width correlates positively with the size or category of a road, as for instance the average width of a district road lane is 3.75 meters whereas the average width of a highway lane is 6.40 meters. Knowing the quantity of lanes present in a given section, as well as the average lane width of the road type, the area sealed by a roadway can be calculated.

Table 5.1: Average width of lane representing the construction of the entire roadway per road category in meters.

\begin{tabular}{|lrrr|}
\hline Road category & $\begin{array}{c}\text { Standard cross- } \\
\text { sections as of RAS-Q }\end{array}$ & $\begin{array}{r}\text { Number } \\
\text { of lanes }\end{array}$ & $\begin{array}{c}\text { Average lane } \\
\text { width }\end{array}$ \\
\hline District road & 7.5 & 2 & 3.75 \\
Country road & 9.5 & 2 & 4.75 \\
Federal highway & $10.5 ; 15.5 ; 20$ & 3,4 & 5.20 \\
Freeway & $26 ; 29.5 ; 33 ; 35.5$ & 4,6 & 6.40 \\
\hline
\end{tabular}




\subsubsection{Accounting for bypasses and resting areas}

The second type of area that has to be accounted for in order to calculate the share of sealed area per user for larger roads is associated "auxiliary area". It mainly consists of various types of bypasses, e.g. ramps or emergency accesses, of junctions, and of resting areas including, fuel stations. Further, but less directly associated areas like the diverse kinds of parking areas for instance of restaurants, at the workplace or at home, are not considered in the present analysis. In the following, first the area sealed by bypasses, and next that sealed by resting areas are determined. The totals will be compared to and proportionately added to the area sealed by roadways alone. This will find expression in a further increase of average lane width per road type, which will then account for the area sealed by a roadway and the auxiliary construction.

In 2011, the length of bypasses in Germany was $4.168 \mathrm{~km}$ for highways and $8.337 \mathrm{~km}$ for freeways (BMVBS 2011). Assuming a more conservative width of bypasses than the average lane width indicated in Table 5.1, widths of 4.5 meters for highways and 5.5 meters for freeways are chosen. Multiplying length by width, the value of area sealed by bypasses of highways is $18.756 \mathrm{~km}^{2}$ and it is $45.854 \mathrm{~km}^{2}$ for freeways. In order to assign these values to the two road types, the areas sealed by the roadways of freeways and highways is calculated. Before referenced statistics of the BMVBS, which indicate total lengths of the major different types of roads of the national road grid, including their distribution by the number of traffic lanes, are used (see Table 5.2).

Table 5.2: Length of federal highways and freeways per number of traffic lanes in $\mathrm{km}$.

\begin{tabular}{|lrrrrrrrrr|}
\hline Number of lanes & $\mathbf{1}$ & $\mathbf{2}$ & $\mathbf{3}$ & $\mathbf{4}$ & $\mathbf{5}$ & $\mathbf{6}$ & $\mathbf{7}$ & $\geq \mathbf{8}$ & Total \\
\hline Km of highway & 42 & 34,373 & 1,439 & 3,641 & 60 & 155 & & 39,710 \\
Km of freeway & 3 & 101 & 71 & 8,993 & 651 & 2,896 & 41 & 63 & 12,819 \\
\hline
\end{tabular}

When calculating areal values from the lengths indicated in Table 5.2, for federal highways an average lane width representing the entire construction of 4.75 meters is chosen. The value is larger than the one adopted to calculate the area sealed by bypasses because for main roads the impact of additional features is assumed to be larger. Yet, the value remains smaller than the average lane width suggested by RAS-Q because a large percentage of smaller roads is assumed not yet to adhere to the standard crosssections as of RAS-Q. As this does not apply to freeways, here the average 6.4 meters as of RAS-Q indicated in Table 5.1 are assumed. This yields areas consumed strictly by roadways of $422.270 \mathrm{~km}^{2}$ for federal highways and $369.997 \mathrm{~km}^{2}$ for freeways. This translates to a percentage of area sealed by bypasses compared to the area sealed strictly by roadways of $12.39 \%$ for freeways and $4.44 \%$ for federal highways. 
To determine the area covered by resting areas, two approaches are pursued and their results are compared. This course of action is chosen because detailed data that quantifies sizes of resting areas for the entire country of Germany is not available. The first approach uses the data collected and made available by a few individual states. The results then will be extrapolated to the national level. Data reported for RhinelandPalatinate indicates that the area covered by resting areas is $1.744 \mathrm{~km}^{2}$ (Kunz 2013). This accounts for $3.16 \%$ of the area covered by freeways and federal highways of the state, which is $55.217 \mathrm{~km}^{2}$ (BMVBS 2011, calculation extended by the author). Compared to the entire area concerned under the category 'road, path, place', which for Rhineland-Palatinate is $987 \mathrm{~km}^{2}$ (Federal Statistical Office 2013b), the percentage of land used by resting areas is $0.18 \%$. To obtain a percentage that is as realistic as possible, likewise calculations were made for other federal states of which detailed data was available, namely Brandenburg, Lower Saxony, and North Rhine-Westphalia (see Table 5.3 and a map of the federal states of Germany in Appendix C).

Table 5.3: Absolute land use values (in $\mathrm{km}^{2}$ ) and proportions of resting areas

\begin{tabular}{|cccccc|}
\hline State & $\begin{array}{c}\text { Rest- } \\
\text { ing } \\
\text { area }\end{array}$ & $\begin{array}{c}\text { Surface of } \\
\text { fed. highways } \\
\text { \& freeways }\end{array}$ & Rest. area & $\begin{array}{c}\text { Surface of } \\
\text { 'road, path, }\end{array}$ & Rest. area \\
\hline $\begin{array}{c}\text { Rhineland- } \\
\text { Palatinate }\end{array}$ & 1.744 & 55.216 & $3.16 \%$ & 987.000 & $0.18 \%$ \\
$\begin{array}{c}\text { Brandenburg } \\
\text { place' }\end{array}$ & 1.604 & 51.686 & $3.10 \%$ & 914.060 & $0.18 \%$ \\
$\begin{array}{c}\text { Lower Saxony } \\
\begin{array}{c}\text { North Rhine- } \\
\text { Westphalia }\end{array}\end{array}$ & 1.368 & 92.212 & $1.48 \%$ & $2,010.000$ & $0.07 \%$ \\
\hline
\end{tabular}

Comparing the size of resting areas either to the surface covered by federal highways and freeways or to the category 'road, path, place', the percentage of land used by resting areas is significantly smaller in Lower Saxony than it is in Rhineland-Palatinate, Brandenburg, and North Rhine-Westphalia. This is probably due to Lower Saxony being a large territorial state that has a significantly smaller density of freeways as traffic spreads out more. This also explains the low resting area/ 'road, path, place' ratio of 0.07. Statistics show that the state has many more federal highways compared to freeways, as the ratio of length is $0.3 \mathrm{~km}$ of freeway per $1 \mathrm{~km}$ of federal highway compared to for instance that number being $0.5 \mathrm{~km}$ of freeway per $1 \mathrm{~km}$ of federal highway in North Rhine-Westphalia. The lower percentage of freeways compared to highways and the area taken up by the entire road grid explains the smaller ratio of resting areas as they are built mainly along freeways. While the plain average of the four resting area/ 'road, path, place' ratios of Table 5.3 is circa 0.14 , with regard to topography and population density, for all of Germany an average share of only $0.11 \%$ of resting area 
per total area of the category 'road, path, place' is assumed. $80 \%$ of the area classified as resting area is assumed to be sealed. The total surface of 'road, path, place' in 2012 having been $15,708.000 \mathrm{~km}^{2}$ (Federal Statistical Office 2013b), this yields an areal value of the surface sealed by resting areas in Germany of $13.823 \mathrm{~km}^{2}$ or $0.9 \%$.

The second approach uses information from the "Guidelines for resting areas of roads" (FGSV 2011). In Germany, there are 1,550 smaller resting areas with or without restrooms and 430 larger service areas with restaurants and/ or filling stations. As of the guidelines, smaller resting areas with less than 40 individual parking sites have a size between 2 and 4 hectares and those that provide up to 80 parking units should have a size between 4 and 5 hectares. Larger service areas with less than 100 parking units should be 4 to 6 hectares in size, areas with more than 100 and less than 200 units are to take up between 6 and 8 hectares, and facilities with more than 200 individual parking units are indicated to be larger than 8 hectares (FGSV 2011). However, this information is of limited use for two reasons: firstly because it indicates target states and does not display the actual size of resting areas, and secondly because no distribution of areas by size other than the overall distribution of there being 1,550 smaller and 430 larger resting areas is known. Since most smaller resting areas are of a size closer to 2 than to 4 hectares, an average value of 2.5 hectares for the group of 1,550 smaller resting areas is assumed. Assuming also a conservative average for larger service areas of 5.5 hectares yields a total value of surface covered by resting areas of $62.400 \mathrm{~km}^{2}$. Supposing that this target state is not attained in practice and that the share of strictly sealed area of resting areas is only between 30 and $45 \%$, the final value of the surface sealed by resting areas shrinks to about $22.000 \mathrm{~km}^{2}$.

When comparing the values of the two approaches, which yielded $13.823 \mathrm{~km}^{2}$ as of the cadaster- data-approach and about $22 \mathrm{~km}^{2}$ as of the guidelines-approach, and when further considering that of the two approaches the cadaster-approach is perceived to be more accurate and hence more reliable, the adopted total value should resemble the result of the cadaster-approach to a higher degree. Therefore for all of Germany the area sealed by resting areas of major roads is assumed approximately to amount to $15,000 \mathrm{~km}^{2}$. This equals $0.10 \%$ of the total area recorded as 'road, path, place'.

The total area sealed by bypasses and resting areas was determined in order to be accounted for when assuming an average lane-width per type of road that considers direct auxiliary construction in addition to the roadway itself. Table 5.4 summarizes the values obtained for the three types of area, roadway, bypass, and resting area. Differentiating by type of road, the area of resting facilities is allocated entirely to freeways, because it is along these roads where it is essentially located. 
Table 5.4: Area sealed in Germany for roadways, bypasses, and resting areas in km².

\begin{tabular}{|c|c|c|c|c|c|}
\hline & \multicolumn{5}{|c|}{ Area sealed by } \\
Type of road & Roadways & Bypasses & $\frac{\text { Bypasses }}{\text { Roadways }}$ & Rest. areas & $\frac{\text { Rest. areas }}{\text { Roadways }}$ \\
\hline $\begin{array}{l}\text { Federal } \\
\text { highway }\end{array}$ & 422.270 & 18.756 & $4.44 \%$ & - & - \\
Freeway & 369.997 & 45.854 & $12.39 \%$ & 15.000 & $4.05 \%$ \\
\hline
\end{tabular}

Average lane-widths assumed when considering roadways alone were 4.75 meters for federal highways and 6.4 meters for freeways. Accounting for the areas covered by bypasses and resting facilities, in equations $5.1 \mathrm{a}$ and $5.1 \mathrm{~b}$ adjusted average lanewidths are calculated. They come out to be $4.96 \mathrm{~m}$ for federal highways and $7.45 \mathrm{~m}$ for freeways. This concludes the approximation of average lane-width per type of road.

Equations 5.1: Average lane-width of federal highways (a) and freeways (b).

a) Final average lane-width of highways $=4.75 \mathrm{~m} \cdot(1+0.0444)=4.96 \mathrm{~m}$

b) Final average lane-width of freeways $=6.40 \mathrm{~m} \cdot(1+0.1239+0.0405)=7.45 \mathrm{~m}$

\subsubsection{Mathematical representation and further input variables}

Knowing the length of a section, the number of lanes, and the type of road, the total sealed area of a road section can be calculated. In order to determine the share of an individual user, in step two, the obtained result is divided by the quantity of vehicles using a section of a road over the course of one year. The result describes the quantity of sealed area that an individual user driving on the road one time in a year is responsible of. One year is assumed to be an appropriate time horizon because it represents a compromise of a road usually being used for various years and the ecological impact to occur not just once but day by day. In addition, the level of use within one year seems significant for infrastructure planning. That is, if a substantial share of users in one year uses a road half as often as before, this is likely to provoke a change in infrastructure planning for the concerned road. While it seems unrealistic that roads would be dismantled to a high degree, a decrease in demand within one year would be a signal not to extend a road, to adopt concepts of temporary closures during a specific time of day or year, or it might render the building of crossing structures obsolete. Moreover, negative environmental impact of a road, for instance in form of the barrier effect or the quantity and reach of substances or noise, would decrease considerably. This could not be accounted for when assuming the total quantity of users from periods much longer than one year. Yearly results are also suitable for users from companies as their 
principle reporting and management periods are one year. If on a given route smaller roads than federal highways or freeways are used, their average lane width shall be assumed to be 4 meters. As this value significantly varies between smaller roads, which were not targeted and analyzed in as much detail as were federal highways and freeways, the precision of the result suffers as the share of smaller roads being used augments. For a route of $n$ homogeneous road sections, where a section is homogeneous if neither the number of lanes, the type of road, nor the volume of traffic change, the before mentioned steps of calculation yield the formula indicated in Equation 5.2. The measuring unit of the result is square meters.

Equation 5.2: Share of area sealed of one trip in one year.

$$
S S A=\sum_{i=1}^{n} \frac{d_{i F W} \cdot 7.45 m \cdot l_{i}+d_{i F H} \cdot 4.96 m \cdot l_{i}+d_{i S R} \cdot 4 m \cdot l_{i}}{d t v_{i} \cdot 365}
$$

$\mathrm{d}_{\mathrm{FF}}=$ distance travelled in section $\mathrm{i}$ on a freeway in meters

$\mathrm{d}_{\mathrm{FH}}=$ distance travelled in section $\mathrm{i}$ on a federal highway in meters

$\mathrm{d}_{\mathrm{iSR}}=$ distance travelled in section i on a low category road ("smaller road") in meters

$\mathrm{i}=$ homogenous section $\mathrm{i}$, with $\mathrm{i}=1,2, \ldots, \mathrm{n}$ (homogenous in quantity of lanes and daily traffic volume)

$\mathrm{m} \quad=$ meter (unit of measure)

$\mathrm{I}_{\mathrm{i}} \quad=$ number of lanes in section $\mathrm{i}$ (if varying, take ruling quantity for entire section)

$\mathrm{dtv}_{\mathrm{i}}=$ average daily traffic volume of section $\mathrm{i}$

$\mathrm{SSA}=$ share of sealed area in $\mathrm{m}$ per trip

One parameter needs to be added to the formula in order to obtain a more precise allocation of impact and in order to obtain a more functional result. It concerns the distinction between different vehicles, because vehicles of different sizes, weights, engines, and speeds have dissimilar demands on a roadway and produce dissimilar degrees of environmental impact. With regard to spatial use and deterioration of road surface, vehicle size is the most relevant factor. In order to account for differing impact factors, the approach to classify different vehicle types by attributing them impact proportionate values expressed in passenger car units (PCUs) evolved. This means that when a standard car has a PCU of 1, for instance a truck equal or larger 12 tons gross vehicle weight (GVW) has a PCU of 3 . This allows fairly describing the proportion of impact of the two different vehicles. PCUs are used in order to standardize vehicles in relation to a passenger car in order accordingly to allocate costs and impacts. A vehicle's PCU is determined by its size, weight, engine power, width of wheels, and speed. In order not to spoil the realistic areal value that is yielded using the above formula, the total sealed area has to represent the sum of passenger car units of all vehicles. 
Since for most roads no data from traffic censuses on the distribution of vehicle types exists, the distribution of vehicle types is deduced from their shares of the national mileage of Germany. Data on national mileages stems from Elsner (2010) for the year 2007 and from Bast (2005) for the year 2002. Elder vehicle type-specific data published by Bast, which is the Federal Highway Research Institute of Germany, is necessary because it differentiates between different sizes of trucks. The data from Elsner does not report this level of detail. A differentiation between trucks of different sizes is viewed necessary because the differences in impact are significant. PCU values were adopted and adjusted from Delft et al. (2011) and from ProgTrans AG and IWW (2007). All relevant information is displayed in Table 5.5. For the vehicle types of trucks and semitrailer tractors further differentiation regarding the differing sizes of vehicles may be possible and would yield more detailed and fairer results. However, to date, the data necessary for such differentiation is not available. It is dismissed to approximate this data because accuracy would not be high and the adopted level of differentiation is viewed sufficient.

Table 5.5: Mileage shares and vehicle factors; adapted from Elsner 2010, Bast 2005, Delft et al. 2011, ProgTrans AG and IWW 2007.

\begin{tabular}{|r|c|c|c|}
\hline \multicolumn{1}{|c|}{$\begin{array}{r}\text { Vehicle type } \\
\left(\mathbf{v}_{\mathbf{x}}\right)\end{array}$} & $\begin{array}{c}\text { National mileage } \\
\text { in billion tkm }\end{array}$ & $\begin{array}{c}\text { National } \\
\text { mileage in \% }\end{array}$ & $\begin{array}{c}\text { Vehicle factor/ } \\
\text { PCU } \mathbf{f}\left(\mathbf{v}_{\mathbf{x}}\right)\end{array}$ \\
\hline $\begin{array}{r}\text { Motorcycles }\left(\mathrm{v}_{\mathrm{m}}\right) \\
\text { Buses }\left(\mathrm{v}_{\mathrm{b}}\right)\end{array}$ & 15.4 & 2.23 & $0.5 \mathrm{f}\left(\mathrm{v}_{\mathrm{m}}\right)$ \\
Cars \& Combination vehicles $\left(\mathrm{v}_{\mathrm{c}}\right)$ & 587.5 & 84.90 & $1.0 \mathrm{f}\left(\mathrm{v}_{\mathrm{c}}\right)$ \\
Pick-up trucks $\leq 3.5$ tons $\left(\mathrm{v}_{\mathrm{t} 3.5}\right)$ & 3.4 & 0.49 & $2.5 \mathrm{f}\left(\mathrm{v}_{\mathrm{b}}\right)$ \\
Trucks $>3.5 \leq 12$ tons $\left(\mathrm{v}_{\mathrm{t} 12}\right)$ & & 5.04 & $1.2 \mathrm{f}\left(\mathrm{v}_{\mathrm{t} 3.5}\right)$ \\
Trucks $>12$ tons $\left(\mathrm{v}_{\mathrm{t}>12}\right)$ & & 1.67 & $2.0 \mathrm{f}\left(\mathrm{v}_{\mathrm{t} 12}\right)$ \\
All trucks combined & & 2.08 & $3.0 \mathrm{f}\left(\mathrm{v}_{\mathrm{t}>12}\right)$ \\
Semitrailer tractors $\left(\mathrm{v}_{\mathrm{st}}\right)$ & 60.8 & 8.79 & \\
Other $\left(\mathrm{v}_{\mathrm{o}}\right)$ & 16.9 & 2.44 & $4.0 \mathrm{f}\left(\mathrm{v}_{\mathrm{st}}\right)$ \\
Total & 8.0 & 1.16 & $1.0 \mathrm{f}\left(\mathrm{v}_{\mathrm{o}}\right)$ \\
\hline
\end{tabular}

For the modification of the formula this means that in order to yield the share of sealed area of one passenger car unit per day, the shares of the distinct vehicle types $\left(v_{x}\right)$ have to be multiplied by the corresponding vehicle factors $\left(f\left(v_{x}\right)\right)$ and be added $\left(\sum_{v_{\mathrm{x}=\mathrm{m}}}^{\mathrm{o}}\left[\right.\right.$ share of $\left.\left.v_{x} \cdot \mathrm{f}\left(\mathrm{v}_{\mathrm{x}}\right)\right]\right)$. As all variables in this term are constant, the resulting value can already be calculated. It is 1.1379 . The value has to be multiplied by the average number of daily user of a section. In order to obtain the share of an individual vehicle of a certain vehicle type, the intermediate result has to be multiplied by the vehicle factor of the respective type. This results in the formulae indicated as Equations 5.3. 
The measuring unit of the result is square meters. Optionally, in order to obtain a serviceable yearly result for reoccurring trips, the result can further be multiplied by the number of trips of a given vehicle on that route during one year.

Equations 5.3: Calculation of share of sealed area per vehicle per route.

a)

b)

$$
S S A=\sum_{i=1}^{n} \frac{d_{i F W} \cdot 7.45 m \cdot l_{i}+d_{i F H} \cdot 4.96 m \cdot l_{i}+d_{i S R} \cdot 4 m \cdot l_{i}}{\left.\mathrm{dtv}_{\mathrm{i}} \cdot \sum_{v_{\mathrm{x}=\mathrm{m}}}^{\mathrm{o}}\left(\operatorname{share~of~} v_{x} \cdot \mathrm{f}\left(\mathrm{v}_{\mathrm{x}}\right)\right)\right] \cdot 365} \cdot \mathrm{f}\left(\mathrm{v}_{\mathrm{x}}\right)
$$

$$
S S A=\sum_{i=1}^{n} \frac{d_{i F W} \cdot 7.45 m \cdot l_{i}+d_{i F H} \cdot 4.96 m \cdot l_{i}+d_{i S R} \cdot 4 m \cdot l_{i}}{\mathrm{dtv}_{\mathrm{i}} \cdot(1.1379) \cdot 365} \cdot \mathrm{f}\left(\mathrm{v}_{\mathrm{x}}\right)
$$

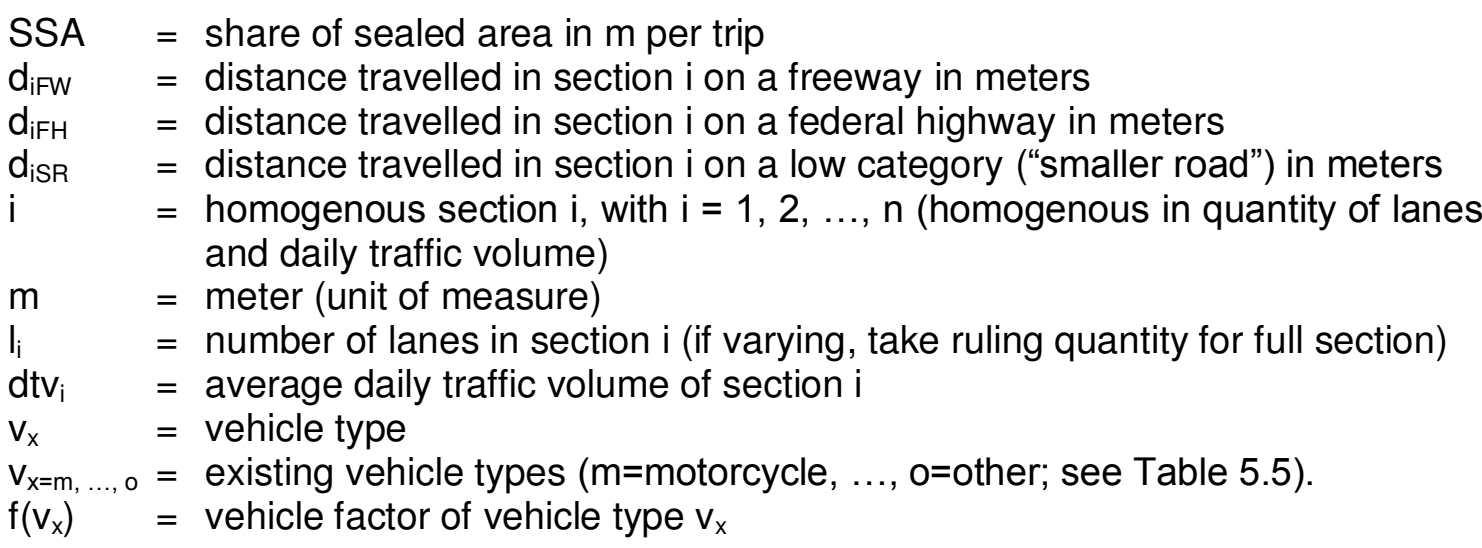

The idea of this method of calculation is to determine the share of sealed area that one individual user is responsible for. While the formula was developed focusing on the impacts of vehicles on higher use roads, parts of a route driven on lower use roads or smaller roads, which are roads of a lower category than federal highways or that carry less than 1,000 vehicles per day, can also be considered (see $d_{i s R}$ ). Here it will usually be sometimes to make an additional assumption concerning the quantity of daily traffic on smaller roads because it is not always metered or the data is difficult to obtain. It is suggested to assume an average daily traffic volume for smaller roads of 2,500 vehicles per day. This average is likely often to be imprecise given the existing variety of intensities of use of smaller roads. Yet, the estimate is chosen and viewed suitable for two reasons: firstly, because it was defined that in order to yield accurate results the formula only be applied to routes with shares of lower use roads below $10 \%$, and secondly because in 2,500 vehicles per day a value is chosen that may be too high for many local roads, but which is viewed suitable in order to avoid yielding a disproportionately high impact of lower use roads that cannot be avoided to be used to a certain degree even when managing the direct spatial impacts of road transports. 
The formula can help companies in their efforts of environmental management because it yields a concrete amount of sealed area that a specific transport is responsible of. A positive trait of the formula is that if a company decides to try and decrease the resulting areal value, this can best be done by lowering haulage distances and by driving on larger and more intensively used roads. The latter behavior matches current strategies of defragmentation that favor the bundling of traffic and then to apply measures that ensure landscape connectivity for the remaining roads (EEA 2011). Before landscape fragmentation is turned to in section 5.2, next a method for calculating the share of degraded area adjacent to a road is presented.

\subsubsection{Determination of the Share of Degraded Area of an Individual User}

As a road is a relatively narrow and hence seemingly area-efficient construction, it may be counterintuitive that the total spatial impact of most traffic types and particularly that of fuel-powered road bound movements in fact is extensive. The spatial impact is less obvious for effects that do not come into effect at immediate vicinity of occurrence, as for instance the spatial demands of input materials that occur along the supply chain or the effects from emitting carbon dioxide like upper ocean warming (IPCC 2013). Spatial impact is more intelligible for more direct impacts on areas surrounding roads, probably also because they are not limited to animals, plants, and ecosystems, but for instance in the cases of particulate matter or noise also concern the well-being of many people. With regard to ecological impacts, some effects like substance emissions, rubber, salt, or other waste, changes in vegetation, or changes in microclimate or water geology predominantly concern the nearby area between 10 to 50 meters of a road (Friedrich \& Geldermann 2013b, Reck \& Kaule 1993). Other impacts like noise, road mortality, or the barrier effect, which can aggravate to a degree of harming flora and fauna at the level of entire populations, represent farther-reaching direct impacts. While the population-level impacts of road mortality, the barrier effect, and also climate change, potentially reach out further than noise, noise straightforwardly alters habitats and disturbs animals for up to several kilometers (Kaseloo 2006, Barber et al. 2010, Shanley \& Pyare 2011, Boarman \& Sazaki 2006). While habitats adjacent to roads and their inhabitants are constantly subject to a variety of disturbances like visual disturbances, air pollutants, sedimentation, changes in microclimate, or attraction to a road with danger emanating from vehicles or predators that make it more difficult to discern disturbance distances (Liu et al. 2008), none of these impacts is regarded severe enough or altogether able to explain the changed behavioral patterns observed in a variety of species (Forman et al. 2002). Other researchers agree that considering both the extension and the magnitude of the before mentioned impacts per traffic unit, noise is assumed to be 
the overwhelming reason for habitat loss near roads and it hence is viewed to be the spatially most extensive direct impact (McClure et al. 2013, Parris \& Schneider 2009). Therefore, the extension of the road-effect zone, which is the reach of significantly degraded area, in this work primarily is measured by the impact of traffic noise.

That the effect of noise on more susceptible species like birds often was identified to be one of the key variables in determining the expansion of the road-effect zone fits this decision (Forman et al. 2002). Moreover, due to the longtime confirmed effects of noise not only on subjective well-being, but also on physiological health in humans, with expected likewise consequences for animals, in the following the extension of noisecorridors that significantly affect animals is analyzed. This allows to determine a land use value that is not limited to measuring the physical location of the road, but that accounts for and hence includes the significantly impacted adjoined area. Various studies found that the reach of impact of noise increases with traffic volume. Thus, the correlation between the two is analyzed in order to determine extensions of degraded areas fairly accurately and according to impact (Reijnen \& Foppen 1995, Forman et al. 2002, Kaseloo 2006, Charry \& Jones 2009). While noise is assumed to be the spatially most extensive impact, a final formula that allows for determining degraded ecological area shall also account for other impacts that have spatial implication. This for instance is the case when no noise pollution occurs due to the presence of noise barriers or low traffic volumes, but when habitat is degraded due to sedimentation or changed microclimate, or due to the population level effects stemming from fragmentation or road mortality.

In the past, there have been attempts to calculate the effective habitat loss from traffic noise, but studies either referred to particular species and sites or, on the opposite, investigated large geographic scales (Forman 2000, Reijnen \& Foppen 2006, Helldin et al. 2013). No method that recognizes average extensions of noise-levels with significant effects on flora and fauna along larger roadways and that allows determining the share of impact of an individual road user in a specific site is known.

Two approaches will be pursued in order to obtain an expression that determines the extension of a certain sound pressure level into the landscape from traffic volume: at first, the existing theoretical physical modeling of determining sound pressure levels from traffic is considered and attempted to be rearranged in order to allow for a direct calculation of noise zone extension with traffic volume and vehicle size being the only input factors. However, the results to be obtained only are estimates and cannot be assumed to yield an exact extension of a certain sound pressure level for a particular location because of the influence of individual topography and climate is significant. 
In a second approach, real data from existing noise calculations is used. In Germany, some federal states measure the extension of noise levels along major roads. The results are communicated through graphical representation in the states' noise viewers (access to noise viewers: MKULV NRW 2014, MELS 2014, BfU 2014). Together with data on traffic volumes and compositions of traffic that stems from the before mentioned Federal Highway Research Institute of Germany (Bast), an average correlation between traffic and sound pressure level extension can be deduced. As both approaches have advantages over each other they shall both be carried out and the results be compared and discussed to obtain a final mathematical expression of noise extension from traffic. In both approaches the calculation of the extension of the noise corridors refers to only one side of the road. Thus, to obtain the total area affected by noise it has to be kept in mind also to consider the effect on the adjacent area on the other side of a road. The goal is to obtain a mathematical expression of the relation of the parameters of traffic volume and noise zone extension, that will allow for a straightforward calculation of the extension of a sound pressure level of $55 \mathrm{~dB}(\mathrm{~A})$ subject to traffic volume.

\subsubsection{The extension of sound pressure level from physical modeling}

In noise calculations it is differentiated between noise emissions that occur at a source and noise immissions that are felt by a third party affected at distance from a source. The technical and more accurate expression for 'noise' is 'sound pressure level'. Sound pressure needs an elastic medium to spread or propagate. Such media can be gases, liquids, or solids; in a vacuum, no spreading of sound pressure is possible. Sound pressure provoked by traffic spreads through the atmosphere because the movement of objects causes vibrations in air molecules which then jolt other molecules (ODOT 2006). Sound pressure declines because parts of the acoustic energy are absorbed by molecular friction and by other characteristics of the molecules of the atmosphere and because in the process, these parts of the acoustic energy are changed into non audible waves of oscillation energy. Commonly, attenuation of sound pressure is understood as the interference of sound propagation by the absorption of airborne sound.

In the most basic noise propagation model used for the calculation of sound pressure level, ' $S P L_{m}^{25}$ ' (see Equation 5.4a), an approximate immission-value in $\mathrm{dB}(\mathrm{A})$ and at 25 meters of distance from a road is calculated (VBUS 2006). In the equation, SPL stands for time-average sound pressure level, 25 indicates the distance from the source in meters, and $\mathrm{m}$ indicates that a normal or medium situation in terms of all relevant variables other than average hourly traffic volume $\left(\mathrm{tv}_{ø \mathrm{~h}}\right)$ and share of heavy duty vehicles $(p)$ is assumed. While the formula reliably determines the immission-value at 25 meters, it is too rudimentary to be used for calculations of immission-values at larger 
distances. This is not because the formula uses average values for the most relevant parameters of the formation and dispersion of sound pressure levels of traffic noise at low distance, such as maximum speed limit, inclination, road surface, free propagation, and height of receiver, but due to the impacts of different triggers of attenuation, such as distance and air, ground, and atmospheric absorption, that are not considered in a way that would allow to deduce immission values for larger distances from the formula. To this end, the extended formula ' $S P L_{m, i}$ ' was developed (Equation $5.4 \mathrm{c}$ ). It builds on $S P L_{m}^{25}$, which becomes ' $S P L_{m, E}$ ' once adjustments for the formerly assumed average values of speed limit $\left(D_{s}\right)$, road surface $\left(D_{r s}\right)$, inclination $\left(D_{i}\right)$, and mirror sound sources $\left(D_{m s}\right)$ are made (see Equation 5.4b). Besides the result of $S P L_{m, E}$, the length of the road as a line source $\left(D_{1}\right)$, distance and air absorption $\left(D_{d a}\right)$, ground and meteorological attenuation $\left(D_{g m}\right)$, barrier attenuation $\left(D_{b a}\right)$, reverberation $\left(D_{\text {rev }}\right)$, and atmospheric attenuation $\left(\mathrm{D}_{\mathrm{at}}\right)$ are variables added in $S P L_{m, i}$. This allows obtaining more precise sound pressure immission levels for larger distances. Despite its inferiority with regard to precision to $S P L_{m, i}$, a change in the underlying assumptions of absorption is viewed to permit using the $S P L_{m}^{25}$-formula in the following calculations. As mentioned above, it contains two traffic related variables, average quantity of vehicles per hour $\left(\mathrm{tv}_{ø \mathrm{~h}}\right)$ and share of heavy duty vehicles (p). Further, it assumes the following average conditions for the most impacting road- and vehicle-related parameters: a non-serrated melted asphalt surface, a speed limit of 100 kilometers per hour, an incline or decline $\leq 5 \%$, a free sound propagation at 2,25 meters, and a height of the reception of immissions of 4 meters (VBUS 2006).

Equations 5.4: Noise propagation models used to calculate immission-sound pressure levels at dissimilar distances (VBUS 2006).
a) $S P L_{m}^{25}=37.3+10 \cdot \log \left[t v_{\emptyset h} \cdot(1+0.082 \cdot p)\right]$
b) $S P L_{m, E}=S P L_{m}^{25}+D_{s}+D_{r s}+D_{i}+D_{m s}$
c) $S P L_{m, i}=S P L_{m, E}+D_{l}-D_{d a}-\max \left[D_{g m}, D_{b a}\right]+D_{r e v}+D_{a t}$

Despite its high accuracy in calculating immission-values at distances larger or much larger than 25 meters, mainly for its input parameters and the respective assembly of equations needed for their calculation, $S P L_{m, i}$ is regarded to be too complex to be used to build a simplified model that can relate traffic volume and noise expansion. In addition, the goal at hand is to obtain an average value of noise emissions from certain traffic volumes that is valid at a wider landscape level and that is not limited to particular geographic conditions. Therefore, the simplified sound pressure propagation model $S P L_{m}^{25}$ will be used, but underlying assumption will be changed in a way as to account 
for distance and the different types of absorption, mainly atmospheric, ground, and meteorological absorption. To noise models on traffic, the following two rules apply:

Rule 1: When doubling the distance from a line source, time-average sound pressure level falls $3 \mathrm{~dB}(\mathrm{~A})$ due to geometric absorption (Dooling \& Popper 2007).

Rule 2: When traffic volume doubles, time-average sound pressure level in 25 meters $\left(S P L_{m}^{25}\right)$ rises $3 \mathrm{~dB}(\mathrm{~A})$ (VBUS 2006).

In order to use $S P L_{m}^{25}$, the first rule will be modified in a way so that it can account for the average impact of atmospheric or air, ground, and meteorological absorption. Atmospheric and meteorological absorption are the parts of transmission loss caused by conversion of acoustic energy into other forms of energy; ground attenuation is the part of transmission loss caused by interaction of the propagating sound with the ground (Barber et al. 2010). With regard to geometrical divergence, which takes into account the atmospheric absorption of the air and the form of the average surface of the planet, which curves and is not straight, sound from a point source, such as a single vehicle, spreads uniformly and the sound pressure level drops off at a rate of $6 \mathrm{~dB}(\mathrm{~A})$ for each doubling of distance. In acoustics this is referred to as the "inverse square law" (Bolt Beranek \& Newman 1973). However, in a highway situation the surroundings are usually not subject to the noise of a single vehicle but to a more or less continuous line of vehicles. For such a line source sound spreads almost cylindrically and the corresponding rate of sound level drop-off falls to $3 \mathrm{~dB}(\mathrm{~A})$ for each doubling of distance (Agent \& Zegeer 1981).

Lamure (1986) considers ground attenuation to be the most important effect with regard to the propagation of noise. Agent and Zegeer (1981) state that regarding the impact of ground attenuation, source height and primarily receiver height, are crucial variables because ground attenuation significantly decreases with source height and receiver height. They recommend to assume a noise drop-off per doubling of distance of $3 \mathrm{~dB}(\mathrm{~A})$ for reflective ground covers, of $4.5 \mathrm{~dB}(\mathrm{~A})$ for absorptive ground covers, and of $6 \mathrm{~dB}(\mathrm{~A})$ for very absorptive ground covers when the receiver height is at or below 3 meters. For receiver heights above three meters a $3 \mathrm{~dB}(\mathrm{~A})$ drop-off per doubling of distance should be used regardless of the type of ground cover (Agent \& Zegeer 1981). Thus, ground bound animals are expected to benefit more from absorption than do aerial species. This explains why many bird species are considered to be among the species most susceptible to noise and why the entire reach of road effects can be determined from changes of behavior in birds. Meteorological attenuation depends on the weather conditions; most relevant are temperature, humidity, and wind. 
While Dooling and Popper (2007) state that in extreme cases total absorption can amount to as much as $5-6 \mathrm{~dB}(\mathrm{~A})$ per doubling of distance, in line with the above findings, Lamure (1986) remarks that it is common practice to assume an overall attenuation of $4 \mathrm{~dB}(\mathrm{~A})$ for each doubling of distance from a traffic induced line source of noise. Sound propagation can also be affected by obstructions like barriers, buildings, or high vegetation. As noise barriers near the source of noise deflect and enlarge the travel of beams of noise and hence lead to lower acoustic energy levels of sound pressure leaving a road, usually all sites near and far to a road benefit from their installation because they receive lower sound pressure levels. Only sometimes can a deflection redirect beams of noise to locations that were impacted less or not reached at all before the installation of sound barriers. Agent and Zegeer (1981) found a very high correlation between noise level and distance, which validated the assumption that traffic noise attenuation is fairly constant per doubling of distance. Overall, despite individual energy absorption values being dependent on the individual geography of a landscape and on the location and height of a receiver, it seems suitable to assume an average propagation in order to estimate average impact. For the above findings and for the implementation of the precautionary principle in the theoretical approach of establishing a formula that calculates the expansion of a certain sound pressure level from traffic volume, it is decided to build on the simplified and with regard to larger distances incomplete model of sound propagation $S P L_{m}^{25}$ and to account for the missing parameters of absorption by assuming an increased drop-off rate of $3.5 \mathrm{~dB}(\mathrm{~A})$ for each doubling of distance. The drop-off rate is kept close to $3 \mathrm{~dB}(\mathrm{~A})$ also because roads more often run through valleys than they run on mountain ranges. This reduces geometric attenuation because the average gradient of the planet, which requires some sound waves to travel as a curve, decreases. This facilitates the propagation of sound pressure waves.

In order to use $S P L_{m}^{25}$, data of two variables is needed. $t v_{ø h}$ is the average hourly traffic volume and $\mathrm{p}$ is the share of heavy duty vehicles. $\mathrm{t}_{\varnothing \mathrm{h}}$ later is substituted by $\mathrm{dtv}$, which is 'average daily traffic volume'. Combining $S P L_{m}^{25}$ and the modification of rule 1 , a formula can be produced that directly calculates the extension of any sound pressure level of noise in meters (Equation 5.5a). Central is the assumption that with every doubling of the distance from the source of noise when beginning at 25 meters a reduction of $3.5 \mathrm{~dB}(\mathrm{~A})$ occurs. This can be continued until a $\mathrm{dB}(\mathrm{A})$-value of zero is reached. However, $0 \mathrm{~dB}(A)$ is defined the lowest threshold of human hearing. Despite variation in the capability of hearing between species, this threshold is assumed similarly to apply to animals. The perception or impact of noise to be disturbing is reached only at higher levels. Reviewing the literature and applying a supposedly more precautionary approach a value of $55 \mathrm{~dB}(\mathrm{~A})$ is chosen (see explanation in the next section). If the extension of a noise zone with noise levels equal or higher than a certain threshold is 
to be calculated, this $\mathrm{dB}(\mathrm{A})$-value is set as a minimum $\left(d B(A)_{M I N}\right)$ and it has to be subtracted from the $\mathrm{dB}(\mathrm{A})$-value calculated at 25 meters distance, which equals the result of $S P L_{m}^{25}$ (Equation5.5b). Accepting and assuming medium values for the relevant variables inherent to $S P L_{m}^{25}$ as they are indicated in the VBUS 2006, this allows to determine the extension of time-average sound pressure levels $\geq 55 \mathrm{~dB}(\mathrm{~A})$ solely by using average hourly traffic volume and share of high duty vehicles as input variables (see Equations 5.5c and 5.5d).

Equations 5.5: Relation of traffic volume, noise immission and SPL extension; own representation using VBUS 2006.

a) Extension of Noise (in meters) $y=25 \cdot 2^{\frac{S P L_{m}^{25} \text { in } d B(A)}{3.5}}$

b) Extension of Noise (in meters) $y=25 \cdot 2^{\frac{S P L_{m}^{25}-d B(A) M I N}{3.5}}$

c) Extension of Noise (in meters) $y=25 \cdot 2^{\frac{\mathrm{SPL}_{\mathrm{m}}^{25}-55}{3.5}}$

d) Extension of Noise (in meters) $y=25 \cdot 2^{\frac{37.3+10 \cdot \log \left[\mathrm{M}_{\phi \mathrm{h}} \cdot(1+0.082 \cdot \mathrm{p})\right]-55}{3.5}}$

\subsubsection{Determination of $55 \mathrm{~dB}(\mathrm{~A})$ as an animal disturbance threshold}

The 'correct' animal disturbance threshold expressed in $\mathrm{dB}(A)$ is difficult to determine because it varies between species and because the frequency of a signal to be duplicated per second (Hertz, where $1 \mathrm{~Hz}$ means 1 cycle per second) might be more relevant than an aggregate $d B(A)$-value. Yet, $d B(A)$ is the common measuring unit of traffic noise. $\mathrm{dB}(\mathrm{A})$ stands for $A$-weighted decibel scale. It sums the sound energy across the frequency spectrum of sounds audible to humans. Sound levels relevant for humans range from $0 \mathrm{~dB}(\mathrm{~A})$, a level which is barely audible, to about $120 \mathrm{~dB}(\mathrm{~A})$, a level at which pain usually is beginning to be felt (ODOT 2006). Since extensive studies and resulting information on the impacts of noise are known for humans, and since biologically the human being is an animal and some of the effects detected to occur in humans have been confirmed to occur in animals, it is no coincidence that the chosen threshold value of disturbance for animals of $55 \mathrm{~dB}(\mathrm{~A})$ lies in the middle of the spectrum of deleterious noise values suggested for humans. The Federal Environmental Agency of Germany (UBA) communicated outside noise thresholds of $65 \mathrm{~dB}(\mathrm{~A})$ during the day and 55 $\mathrm{dB}(\mathrm{A})$ at night to significantly increase cardiovascular disorders (UBA 2012). Also the German Federal Parliament in 1999 advocated nighttime values $\geq 55 \mathrm{~dB}(\mathrm{~A})$ as problematic. The WHO in 2009 in their "Night Noise Guidelines for Europe" recommended $40 \mathrm{~dB}(\mathrm{~A})$ to be the maximum night-noise level outside ( $\left.L_{\text {night, outside }}\right)$ (WHO 2009). The WHO further proposed $55 \mathrm{~dB}(\mathrm{~A})$ as an interim target for countries, where for various 
reasons this value could not be reached at short term. In a different publication, the Federal Environmental Agency of Germany suggests threshold values of $55 \mathrm{~dB}(\mathrm{~A})$ during the day and $45 \mathrm{~dB}(\mathrm{~A})$ at night, both outside and with an ajar window, because, inter alia, sleep disorders would begin to increase significantly at a level of $45 \mathrm{~dB}(\mathrm{~A})$ (UBA 2006). In Germany, the legal thresholds for traffic noise when building a new road are at $59 \mathrm{~dB}(\mathrm{~A})$ during the day and $49 \mathrm{~dB}(\mathrm{~A})$ at night for residential areas (BImSchV 16). In order better to comprehend the threshold-values referred to above, see Figure 5.2 for an overview of typical sound pressure levels that occur in the human environment.

\begin{tabular}{|c|c|c|}
\hline Outdoor Activities & Noise Level $\mathrm{dB}(\mathrm{A})$ & Indoor Activities \\
\hline Gunshot near muzzle & $-160-$ & \\
\hline Jet fighter at 7 meters & $-130-$ & $\begin{array}{l}\text { max. Walkman loudness; } \\
\text { threshold of noise pain }\end{array}$ \\
\hline Aircraft at 7 meters & $-120-$ & $\begin{array}{l}\text { begin of threshold of noise } \\
\text { pain }\end{array}$ \\
\hline Power drill & $-110-$ & loud club or concert \\
\hline Buzz saw & $-100-$ & Trombone choir \\
\hline Truck engine at 5 meters & $-90-$ & \\
\hline Car at $50 \mathrm{~km} / \mathrm{h}$ at 1 meter & $-80-$ & max. vocal loudness \\
\hline $\begin{array}{l}\text { Noisy urban area } \\
\text { Lawn mower }\end{array}$ & $-70-$ & $\begin{array}{l}\text { Vacuum cleaner at } 3 \text { me- } \\
\text { ters }\end{array}$ \\
\hline $\begin{array}{l}\text { Commercial area } \\
\text { Heavy traffic at } 90 \text { meters }\end{array}$ & $-60-$ & Colloquial speech \\
\hline $\begin{array}{l}\text { Quiet urban area } \\
\text { Residential area }\end{array}$ & $-50-$ & $\begin{array}{l}\text { Rather quiet radio music } \\
\text { Speech in living room }\end{array}$ \\
\hline Quiet urban nighttime & & Quiet radio music \\
\hline Quiet suburban nighttime & $-40-$ & $\begin{array}{l}\text { Conference room (back- } \\
\text { ground) }\end{array}$ \\
\hline \multirow[t]{2}{*}{ Quiet rural nighttime } & $-30-$ & $\begin{array}{l}\text { Library } \\
\text { Bedroom at night } \\
\text { Whisper }\end{array}$ \\
\hline & $-20-$ & Trickling water tap \\
\hline $\begin{array}{l}\text { Low rustling leaves in a } \\
\text { forest }\end{array}$ & $-10-$ & Broadcasting studio \\
\hline $\begin{array}{l}\text { Lowest threshold of human } \\
\text { hearing }\end{array}$ & $-0-$ & $\begin{array}{l}\text { Lowest threshold of human } \\
\text { hearing }\end{array}$ \\
\hline
\end{tabular}

Figure 5.2: Examples of sound pressure levels in the human environment; adapted from Caltrans 1998, German Federal Parliament 1999. 
With regard to animals, studies found noise to affect most animal species, among them avian, mammalian, and amphibian species like birds, primates, elk or anurans (Reijnen et al. 1995, Brumm et al. 2004, Gagnon et al. 2007, cp. Eigenbrod et al. 2009). Animal species use acoustic signals for various reasons, such as attracting mates, defending territories, maintaining group cohesion and pair bonds, warning for predators, and hunting (Brumm \& Slabbekoorn 2005). The effects of noise on birds are particularly well investigated, probably because animal densities are relatively easy to be tracked, because vocal communication via sound is particularly common and abundant in some bird species, because the A-weighting, also approximates the shapes of hearing threshold curves in birds, and because avian species are among the more susceptible species, which is helpful in order to determine the near total zone of disturbance from traffic noise (McClure et al. 2013, Helldin et al. 2013, Parris \& Schneider 2009, Barber et al. 2010). However, while $\mathrm{dB}(\mathrm{A})$ is easiest to measure, a more accurate measure is the spectrum level of noise, which is defined as the energy level for each frequency in the sound and which is measured in $\mathrm{Hz}$ and $\mathrm{kHz}$. While most energy in traffic noise lies below about $1 \mathrm{kHz}$ and the spectrum slopes downward from above $1 \mathrm{kHz}$, the frequency region where birds vocalize most and hear best is between $2-4 \mathrm{kHz}$. Thus, estimating the spectrum level in the region of $2-4 \mathrm{kHz}$ from an overall level, such as $\mathrm{dB}(\mathrm{A})$, overestimates the energy and the noise level in the frequency region where birds communicate most (cp. Dooling \& Popper 2007). Yet, for empirical studies on animal density that observe behavioral reactions of animals, it is suitable to correlate density to $\mathrm{dB}(\mathrm{A})$-values. Assuming animal density as the main measure of response means that a study applies a threshold-type approach. This may be a problem because using density as response is unlikely to reveal potential long-term effects on remaining, seemingly unaffected individuals. Impact can still be negative and may be of physiological type or come to effect over time, for instance as hearing loss or changed vocalization.

In the past, in scientific studies usually a noise level of $60 \mathrm{~dB}(\mathrm{~A})$ was assumed to begin to affect the behavior of birds. This is due to the fact that traffic noise of an overall level of $60 \mathrm{~dB}(\mathrm{~A})$ will have a spectrum level of noise around $3 \mathrm{kHz}$ of about $10 \mathrm{~dB}$ SPL, which is roughly equivalent to the spectrum level of noise at $3 \mathrm{kHz}$ in a quiet rural to suburban environment with approximately 40-50 dB(A) ambient noise. This means that beyond traffic noise levels of $60 \mathrm{~dB}(\mathrm{~A})$ birds would have to mask, that is to increase the threshold of detection of sound due to the presence of the aggregate of other sounds, to a higher degree than under noise levels experienced in quiet rural to suburban areas (cp. Dooling \& Popper 2007). However, Dooling and Popper (2007) suggest that as birds require better signal-to-noise ratios than humans in order to discriminate and recognize sounds, that is to communicate effectively, a lower noise level guideline of 55 $\mathrm{dB}(\mathrm{A})$ was probably safer and more realistic. This is supported by Reijnen et al. (1997), 
who found threshold values for traffic noise of breeding birds to be as low as $42 \mathrm{~dB}(\mathrm{~A})$. In addition, while noise levels only above $60 \mathrm{~dB}(\mathrm{~A})$ might significantly impede intentional communication and the perception of adventitious sounds, physiological effects of noise exposure with high certainty can be assumed to be present or to begin at levels of $55 \mathrm{~dB}(\mathrm{~A})$. Helldin et al. (2013) assumed $55 \mathrm{~dB}(\mathrm{~A})$ as a threshold value, implying an average decrease in habitat quality for birds of $70 \%$. Reijnen et al. (1996) found impacts at this level to prompt a significant behavioral response (see Figure 5.3). That humans have better auditory sensitivity than birds might mean that humans are easier disturbed by noise, but it also suggests that communication for birds can be more severely impeded by similar or lower levels of sound pressure (cp. Dooling \& Popper 2007). Overall, in this work $55 \mathrm{~dB}(\mathrm{~A})$ is assumed as a sound pressure threshold of significant ecological impact. The strong orientation towards impacts of noise on avian species, due to their level of susceptibility and the quantity of conducted studies is regarded admissible,

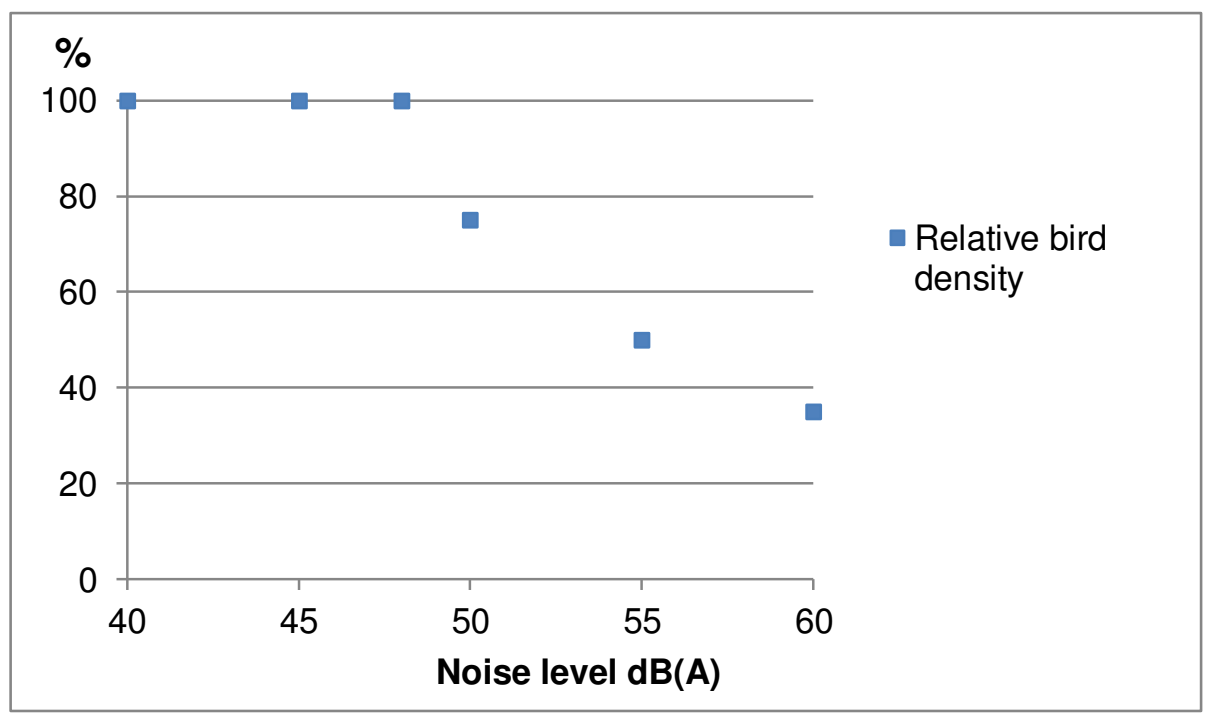

Figure 5.3: Effects of noise on wild bird fauna: relative frequency of breeding birds in Dutch grasslands, adopted from Helldin et al. 2013 and Reijnen et al. 1996.

\subsubsection{Consideration of traffic volume variations within a 24 hour day}

In the basic time-average sound pressure level formula $S P L_{m}^{25}$ (Equation 5.4a), average hourly traffic volume $\left(\mathrm{tv}_{ø \mathrm{~h}}\right)$ is one of the two input variables. Hence, the resulting value is valid only for that particular hour. However the distinction made in the corresponding 24-hour formula ' $L_{D E N}$ ' is not between hours but between times of the day, namely 'day' (06:00 a.m. - 06:00 p.m.), 'evening' (06:00 p.m. - 10:00 p.m.) and 'night' (10:00 p.m. 06:00 a.m.), so that three different $\mathrm{tv}_{ø \mathrm{~h}}$-values are required. The reason behind this is that traffic volume usually does not spread evenly during 24 hours and that the impact 
of noise on humans is considered relevant to a degree as to differentiate between times of day and to use fairly accurate data, particularly for day- and nighttime, when dissimilar levels of noise are detrimental. Also, the European Noise Directive that came into effect in 2002 requires member states to report sound pressure levels by the above stated differentiation of daytimes (EU 2002). A cumulative value for the entire day $\left(L_{D E N}\right)$ is usually reported as well (see Equation 5.6 ). In the formula of $L_{D E N}$, the immission-values for evening and night are subject to an addition of 5 and $10 \mathrm{~dB}(\mathrm{~A})$ respectively. This is done in order to account for the assumption that evening and nighttime noise has a particularly severe impact on human health. Without the adjustment, an $L_{D E N}$-value would fail to express this.

Equation 5.6: Formula to obtain the daily immission value $L_{D E N}$ (VBUS 2006).

$$
L_{D E N}=10 \cdot \log \frac{1}{24}\left(12 \cdot 10^{\frac{L_{\text {Day }}}{10}}+4 \cdot 10^{\frac{L_{\text {Evening }}+5}{10}}+8 \cdot 10^{\frac{L_{N i g h t}+10}{10}}\right)
$$

However, with regard to fauna, various animal species are differently affected at various times of day and at various times of the year in different locations. Therefore, it is difficult and possibly of little use to determine average periods when the majority of species is most negatively affected. Therefore, the weighting of the $L_{D E N}$-formula is not considered and the purely energetic average sound pressure level as of Equation 5.5d is calculated. In the following two paragraphs, two reasons due to which a differentiation of daytimes is regarded needless are explained in more detail.

Firstly, the ratio between positively increased accuracy and negatively increased complexity and negatively decreased user-friendliness is viewed to be negative. A differentiation by daytimes would require users to prepare much more detailed input data. It would no longer be sufficient to know the route of a vehicle, but also the times at which a vehicle was located in a certain place needed to be known. While the generation of the data might not be difficult because most vehicles today are equipped with global positioning systems (GPS), the processing and entry of the data would be much more time consuming as long as no information technology support existed.

The second reason is that it is uncertain whether a differentiation of land use from noise per time of day is of significant relevance in order to achieve a reduction of loss of ecologically valuable area and species' habitats. Although most animal species are particularly active at dusk, night, and dawn, many species are also affected during the day (Blickley \& Patricelli 2010). A calculation of different extensions of noise zones per time of day only becomes relevant, firstly, when ecological values on the degrees of faunal affectedness per time of day are known, and secondly, when there has been 
sufficient moral discussion in society, what a possible utilitarian evaluation of those degrees of affectedness should look like or whether this kind of evaluation is admissible at all. For the human being, for instance in Germany and Europe, the Kantian principles of unalienable rights of the individual have prevailed. Although an approximate transfer of those values to the relationship between humans and non-human animals is viewed reasonable and would suggest a differentiation between daytimes to be lapsed, it does not fully exclude a certain consciousness and coordination in decision-making, potentially to avoid and prioritize traffic during certain times of the day or times of the year.

\subsubsection{Input data needed to calculate noise zone width}

To calculate the extension of the average noise zone formed by road traffic, data on traffic volume ( $\left(v_{ø h}\right.$ or $\mathrm{dtv}$ ) and the share of heavy duty vehicles $(p)$ has to be gathered. For the calculation of time-average sound pressure levels by federal institutions in Germany, data as indicated in Table 5.6 is used whenever more precise information is unavailable. This is uniformly the case for hourly traffic volumes and the corresponding shares of heavy duty vehicles because they are seldom reported by time of day. However, this is to become irrelevant for the calculation at hand, because average daily traffic volume will be used as an input variable once the final formula is developed. Table 5.6 is included nonetheless, in case Equations 5.7b and 5.7c are to be used to calculate the extension of noise zones also for country roads and local roads because their shares of heavy duty vehicles are not always reported or not easily available.

Table 5.6: Average values of hourly daily traffic volume $\left(\mathrm{tv}_{ø \mathrm{~h}}\right)$ and share of heavy duty vehicles (p) for different types of roads and times during the day; source: VBUS 2006.

\begin{tabular}{|c|c|c|c|c|c|c|}
\hline \multirow{2}{*}{$\begin{array}{l}\text { Time of day } \\
\text { Type of road }\end{array}$} & \multicolumn{2}{|c|}{$\begin{array}{c}\text { by day } \\
(6: 00-18: 00)\end{array}$} & \multicolumn{2}{|c|}{$\begin{array}{l}\text { by evening } \\
(18: 00-22: 00)\end{array}$} & \multicolumn{2}{|c|}{$\begin{array}{c}\text { by night } \\
(22: 00-6: 00)\end{array}$} \\
\hline & $\mathrm{tv}_{\text {øh }}(\mathrm{veh} / \mathrm{h})$ & $p(\%)$ & $\operatorname{tv}_{\text {øh }}(\operatorname{veh} / \mathrm{h})$ & $p(\%)$ & $\mathrm{tv}_{\text {øh }}(\mathrm{veh} / \mathrm{h})$ & $p(\%)$ \\
\hline Freeway & $0.062 \mathrm{dtv}$ & 25 & $0.042 \mathrm{dtv}$ & 35 & $0.014 \mathrm{dtv}$ & 45 \\
\hline $\begin{array}{l}\text { Federal high- } \\
\text { way (arterial) }\end{array}$ & $0.062 \mathrm{dtv}$ & 20 & $0.042 \mathrm{dtv}$ & 20 & $0.011 \mathrm{dtv}$ & 20 \\
\hline $\begin{array}{l}\text { Country road } \\
\text { (collector) }\end{array}$ & $0.062 \mathrm{dtv}$ & 20 & $0.042 \mathrm{dtv}$ & 15 & $0.008 \mathrm{dtv}$ & 10 \\
\hline Local road & $0.062 \mathrm{dtv}$ & 10 & $0.042 \mathrm{dtv}$ & 6.5 & $0.011 \mathrm{dtv}$ & 3 \\
\hline
\end{tabular}

When adding the percentages of hourly traffic volume for the three times of day, a value of $102.4 \%$ is reached. There is no intention behind this and it is a pure rounding inaccuracy that is viewed tolerable with regard to the multitude of variables used and 
assumptions made in the noise zone formulae. In this work shares of heavy duty vehicles for freeways and federal highways, which are the two types of roads of particular focus, will not be used as indicated in Table 5.6 because more precise and more recent data from the Federal Highway Research Institute of Germany (Bast) is available. The most recent data is of the year 2012 and stems from fixed automatic counting stations. However, the 718 counting stations on freeways and the 774 stations on federal highways by far do not account for the total number of sections in freeways and federal highways in Germany. Data on all sections is available from a manual traffic census of the year 2010 (Bast 2011a, 2011b). It includes shares of heavy duty vehicles and daily traffic volumes. Where data is missing, average values of the share of heavy duty vehicles on freeways and federal highways issued in a different publication of the Federal Highway Research Institute are used (Fitschen \& Nordmann 2012) (see Table 5.7). In order to mitigate fluctuations the mean value of the years 2009 and 2010 is adopted.

Table 5.7: Average shares of heavy duty vehicles in German freeways and federal highways.

\begin{tabular}{|lrcc|}
\hline Type of road & $\mathbf{2 0 1 0}$ & $\mathbf{2 0 0 9}$ & Mean 2009, 2010 \\
\hline Federal highway & $8.58 \%$ & $11.63 \%$ & $10.11 \%$ \\
Freeway & $14.90 \%$ & $14.21 \%$ & $14.56 \%$ \\
\hline
\end{tabular}

\subsubsection{Noise zone formulae derived from theoretical physical modeling}

At this point, both parameters relevant for the calculation of traffic noise in $\mathrm{dB}(\mathrm{A})$ and the respective extension of sound pressure level are determined and could be fed into formula 5.5d. However, before doing so, the equation is partially solved and simplified.

Equation 5.7: Formation of a simplified formula to determine noise zone extension from traffic volume and the share of heavy duty vehicles.
a) Extension of Noise (in meters) $y=25 \cdot 2^{\frac{\mathrm{SPL}_{m}^{25}-55}{3.5}}$
or Extension of Noise (in meters) $y=25 \cdot 2 \frac{37.3+10 \cdot \log \left[t v_{\emptyset h} \cdot(1+0.082 \cdot p)\right]-55}{3.5}$

The equation can be shortened to:
b) Extension of Noise (in meters) $y=25 \cdot 2 \frac{-17.7+10 \cdot \log \left[t v_{\emptyset h} \cdot(1+0.082 \cdot p)\right]}{3.5}$
$\Leftrightarrow \quad$ Extension of Noise (in meters) $y=25 \cdot 2^{-5.0571+\frac{10}{3.5} \log _{10}(2) \cdot \log _{2}\left[t v_{\emptyset h} \cdot(1+0.082 \cdot p)\right]}$
$\Leftrightarrow \quad$ Extension of Noise (in meters) $y=25 \cdot 2^{-5.0571+0.86 \cdot \log _{2}\left[\operatorname{tv}_{\varnothing h} \cdot(1+0.082 \cdot p)\right]}$
$\Leftrightarrow \quad$ Extension of Noise (in meters) $y=0.7509 \cdot 2^{0.86 \cdot \log _{2}\left[t v_{ø h} \cdot(1+0.082 \cdot p)\right]}$ 
$\Leftrightarrow$ Extension of Noise (in meters) $y=0.7509 \cdot\left[t v_{\emptyset h} \cdot(1+0.082 \cdot p)\right]^{0.86}$

Since data on traffic volumes is usually recorded per day, and since $t v_{\emptyset h}=\frac{d t v}{24}$, the formula can be adjusted as follows:

c) Extension of Noise (in meters) $=0.7509 \cdot\left[\frac{d t v}{24} \cdot(1+0.082 \cdot p)\right]^{0.86}$

If mean shares of heavy duty vehicles per type of road as indicated in Table 5.7 are assumed, the formula for instance for freeways can be shortened further to:

d) Extension of Noise (in meters) $=0.7509 \cdot\left[\frac{\mathrm{dtv}}{24} \cdot(1+0.082 \cdot 0.1456)\right]^{0.86}$

$\Leftrightarrow$ Extension of Noise (in meters) $\mathrm{y}=0.7509 \cdot\left(1.0119 \cdot \frac{\mathrm{dtv}}{24}\right)^{0.86}$

$\Leftrightarrow \quad$ Extension of Noise (in meters) $\mathrm{y}=0.7509 \cdot 1.0102 \cdot\left(\frac{\mathrm{dtv}}{24}\right)^{0.86}$

$\Leftrightarrow \quad$ Extension of Noise (in meters) $\mathrm{y}=0.7586 \cdot\left(\frac{\mathrm{dtv}}{24}\right)^{0.86}$

$\Leftrightarrow$ Extension of Noise (in meters) $\mathrm{y}=0.7586 \cdot \frac{\mathrm{dtv}^{0.86}}{24^{0.86}}$

$\Leftrightarrow \quad$ Extension of Noise (in meters) $\mathrm{y}=0.0493 \cdot \mathrm{dtv} \mathrm{v}^{0.86}$

Inserting the mean of the share of heavy duty vehicles of federal highways, which is $10.11 \%$ (see Table 5.7), an only marginally deviating function develops:

e) Extension of Noise (in meters) $=0.7509 \cdot\left[\frac{\mathrm{dtv}}{24} \cdot(1+0.082 \cdot 0.1011)\right]^{0.86}$

$\Leftrightarrow \quad$ Extension of Noise (in meters) $\mathrm{y}=0.0492 \cdot \mathrm{dtv}^{0.86}$

The resulting Equations 5.7d and 5.7e are power functions. The exponent is smaller than one, which means that the impact of one additional vehicle decreases as the total quantity of vehicles increases. It can be determined that for freeways and federal highways uniformly, at about 200 vehicles an extra vehicle extends the noise zone of a sound pressure level $\geq 55 \mathrm{~dB}(\mathrm{~A})$ by 2 centimeters. This value continually drops to 1,6 $\mathrm{cm}$ at 5,000 vehicles, to $1 \mathrm{~cm}$ at about 30,000 vehicles, to $0.9 \mathrm{~cm}$ at 65,000 vehicles, and to $0.8 \mathrm{~cm}$ at 150,000 vehicles (see Table 5.8). With regard to the sum of all road bound movements, this means that it is 'noise-efficient' to have more traffic on fewer roads than to spread traffic across a dense road grid. This is relevant for the individual user who may be looking to produce the least necessary spatial impact. Also, some forms of mitigation, such as the construction of noise barriers, are more feasible and efficient for fewer and more intensively used roads. 
Table 5.8: Decreasing accession of the noise zone per additional vehicle as of the formulae for both federal highways and freeways.

\begin{tabular}{|cc|}
\hline $\begin{array}{c}\text { Daily traffic } \\
\text { volume }\end{array}$ & $\begin{array}{c}\text { Increase of noise zone in } \\
\text { centimeters }\end{array}$ \\
\hline 200 & 2,0 \\
1,000 & 1.6 \\
5,000 & 1.3 \\
15,000 & 1.1 \\
30,000 & 1.0 \\
65,000 & 0.9 \\
150,000 & 0.8 \\
\hline
\end{tabular}

Concerning the values obtained for the extension of the sound pressure corridor $\geq 55 \mathrm{~dB}(\mathrm{~A})$ for a number of theoretically assumed daily traffic volumes, the first impression is that the formula yields realistic values because they approximately accord to results published by Reijnen et al. (1996) and Forman et al. (2002) (see Table 5.9). Reijnen et al. found birds in grasslands and woodlands to be affected for between 125 and 190 meters at 10,000 vehicles per day and for 365 and 560 meters at 50,000 vehicles. Forman et al. found no significant effect on birds for traffic volumes of up to 8,000 vehicles per day, they measured roughly 400 meters of impact at up to 15,000 vehicles, 700 meters of impact at 30,000, and as much as 1,200 meters of impact for roads being used by more than 30,000 vehicles per day. Eigenbrod et al. (2009) found the value of wetlands likely to be very low for anuran populations if they are within 250 meters of a major highway and hence suggest buffer distances between busy roads and wetlands to amount to between 500 and 2,000 meters. While the results of the studies are consistent among each other as well as with the values yielded by the theoretically deduced formula measuring noise zone extension, it shall be noted that they account for the impact of noise exclusively and that particularly for lower traffic volumes at or below 10,000 vehicles per day, other impacts of traffic are viewed to have larger spatial impacts than noise. Table 5.9 also shows the only marginal variation of results between the equations for freeways and federal highways that occur due to differing average shares of heavy duty vehicles. Further analysis of the results yielded by these equations, which were generated pursuing a theoretical and technical approach, is to follow after having developed an expression and having calculated corresponding results when pursuing a practical approach. Under the practical approach, a mathematical expression that allows determining noise zone extension subject to traffic volume is built from a data sample of existing noise zones. To conclude this section, Table 5.9 indicates the extension of sound pressure levels $\geq 55 \mathrm{~dB}(\mathrm{~A})$ for differing daily traffic volumes that are yielded by the formulae obtained for federal highways and freeways. 
Table 5.9: Average extension of sound pressure levels $\geq 55 \mathrm{~dB}(\mathrm{~A})$ in meters subject to average daily traffic volume yielded by the formulae of the theoretical approach.

\begin{tabular}{|ccc|}
\hline $\begin{array}{c}\text { Daily traffic } \\
\text { volume }\end{array}$ & $\begin{array}{c}\text { Noise zone of freeways, } \\
\text { with } \mathrm{y}=0.0493 \cdot \mathrm{dtv}^{0.86}\end{array}$ & $\begin{array}{c}\text { Noise zone of highways, } \\
\text { with } \mathrm{y}=0.0492 \cdot \mathrm{dtv}^{0.86}\end{array}$ \\
\hline 100 & 3 & 3 \\
500 & 10 & 10 \\
1,000 & 19 & 19 \\
3,000 & 48 & 48 \\
5,000 & 75 & 75 \\
10,000 & 136 & 136 \\
20,000 & 246 & 246 \\
40,000 & 447 & 446 \\
50,000 & 542 & 541 \\
70,000 & 724 & 722 \\
80,000 & 812 & 810 \\
100,000 & 984 & 982 \\
120,000 & 1,151 & 1,148 \\
140,000 & 1,314 & 1,311 \\
\hline
\end{tabular}

In the next section, a real data sample of the extension of noise zones along roads is used to test for a reliable correlation with traffic volume. Results of that equation will be compared to the ones obtained from theoretical modeling in order to possibly combine strengths of the two approaches to build or decide for the most reliable expression. This expression will be suggested to be used in environmental management.

\subsubsection{Development of a correlation between noise zone width and traffic volume from a real data sample}

When using the noise zone data publicly disclosed by the federal states, two major aspects for which the results are expected to differ from the values calculated with the time-average sound pressure level model have to be considered. Firstly, the federal environmental agencies calculate two values, one for nights $\left(L_{\text {Night }}\right)$ and another for the average extension of a noise corridor during 24 hours $\left(L_{D E N}\right)$. For the question at hand it is more appropriate to use the value that considers the total amount of traffic during a day. However, as explained above, the day-evening-night value $L_{D E N}$ does not calculate the factual energetic average but discriminates in favor of evening- and nighttime-noise because the impact on human health is viewed to be most severe during these times of day. Therefore, the purely energetic values calculated using $S P L_{m}^{25}$ are expected to yield narrower noise corridors than those disclosed as $L_{D E N}$ by the federal states. 
The second aspect that might yield a difference is that the calculations of the states account for the topology of the particular surroundings and landscape as well as the average yearly meteorological conditions. Here it could show whether assuming an average reduction of $3.5 \mathrm{~dB}(\mathrm{~A})$ per doubling of distance is appropriate or whether it is significantly off the average attenuation in the landscape. The damping effects of urban areas, which were largely disregarded under the average landscape scenario of the theoretical approach, will not be the reason of significantly deviating results, because when gathering the data it was attempted to collect data in open landscapes that resemble the average situation assumed in the time-average sound pressure level formula. However, this means that using the resulting values to express the correlation between traffic volume and noise extension and applying the results to all locations of all major roads is likely to overestimate the total spatial impact of noise for the threshold level of $55 \mathrm{~dB}(\mathrm{~A})$.

In addition, as this work investigates the effects of road traffic on non-human nature, that is flora and fauna, not to subtract the areas where roads run through commercial or urban and hence non-natural area can be viewed as a significant inaccuracy because here no habitat of meaningful quality is affected. However, as large traffic roads and the construction of bypasses, were and still are attempted not to run through urban area, an analysis of road courses from maps suggests that the overall area adjacent to larger roads that is not at least semi-natural is very likely to be below $2 \%$ overall. In addition, while this work strongly focuses on the impacts of roads on flora and fauna, it must not necessarily be limited to it. Hence, not subtracting urban areas allows partially accounting for the effects of noise on humans. This course of action is endorsed by the precautionary principle, which is underlying this work, and which assumes it to be more suitable to potentially slightly overestimate an impact than to underestimate it.

Data on the extension of corridors of sound pressure levels $\geq 55 \mathrm{~dB}(\mathrm{~A})$ is collected from the noise viewers of three federal states of Germany; North Rhine-Westphalia, Lower Saxony, and Bavaria (MKULV NRW 2014, MELS 2014, BfU 2014). Data on traffic volumes comes from the manual traffic census of the year 2010 of the Federal Highway Research Institute (Bast 2011a, 2011b). The data sample contains freeways and federal highways. As the data sample of the noise viewers already accounts for the impact of heavy duty vehicles, the resulting mathematical expression will not allow for further modeling of neither individual shares of heavy duty vehicles per road nor per road type. Differentiating by states, the quantitative expressions for the correlation between traffic volume and the extension of the noise zone $\geq 55 \mathrm{~dB}(\mathrm{~A})$ indicated in Equations 5.8 are obtained. Differences in the equations are assumed to be the result of, on average, slightly dissimilar values of the input variables of the noise extension formulae, such as average speed limit, road surface, barrier attenuation, reverberation, or ground and 
meteorological attenuation. Topography is largely eliminated as a bias, because data is intentionally collected only in even and mostly open landscapes.

Equations 5.8: Mathematical expressions of the correlation between traffic volume and extension of the noise zone from real data samples and by state.
a) Lower Saxony:
Extension of Noise (in meters) $y=0.0544 d t v^{0.8724}$
b) North Rhine-Westphalia: Extension of Noise (in meters) $y=0.8596 d t v^{0.6088}$
c) Bavaria:
Extension of Noise (in meters) $y=1.4141 d t v^{0.5502}$

When calculating noise extension values for exemplary traffic volumes, results indicated in Table 5.10 show that particularly for rather low and rather high traffic volumes like $500,5,000$, or $100,000 \mathrm{dtv}$, the formulae of the states yield deviating results. For medium traffic volumes like 20,000 or $50,000 \mathrm{dtv}$ the formulae yield comparable values.

Table 5.10: Average extension of sound pressure levels $\geq 55 \mathrm{~dB}(\mathrm{~A})$ in meters.

\begin{tabular}{|rccc|}
\hline $\begin{array}{c}\text { Daily traffic } \\
\text { volume }\end{array}$ & $\begin{array}{c}\text { Lower Saxony, } \\
\mathrm{y}=0.0544 \mathrm{dtv}^{0.8724}\end{array}$ & $\begin{array}{c}\text { North Rhine-Westphalia, } \\
\mathrm{y}=0.8596 \mathrm{dtv}^{0.6088}\end{array}$ & $\begin{array}{c}\text { Bavaria, } \\
\mathrm{y}=1.4141 \mathrm{dtv}^{0.5502}\end{array}$ \\
\hline 100 & 14 & 3 & 18 \\
500 & 38 & 12 & 43 \\
1,000 & 58 & 23 & 63 \\
3,000 & 113 & 59 & 116 \\
5,000 & 154 & 92 & 153 \\
10,000 & 234 & 168 & 225 \\
20,000 & 357 & 307 & 329 \\
40,000 & 545 & 563 & 481 \\
50,000 & 624 & 684 & 544 \\
70,000 & 766 & 917 & 655 \\
80,000 & 830 & 1,031 & 705 \\
100,000 & 951 & 1,252 & 797 \\
120,000 & 1,063 & 1,468 & 881 \\
140,000 & 1,168 & 1,679 & 959 \\
\hline
\end{tabular}

The formulae of Lower Saxony and Bavaria yield comparable results. Despite the deviation of the results for North Rhine-Westphalia being notable, the overall deviation of the results is viewed acceptable to build one formula that represents the data sample as a whole. Moreover, while still within the continuum of use, which ranges from 100 to 150,000 vehicles per day, few roads in Germany carry traffic volumes of 100,000 dtv or higher. This means that the deviation at this level of traffic can be accepted. Equation 
5.9 shows the expression obtained when considering all data in one sample. The data is plotted and the graph of the aggregate formula is shown in Figure 5.4.

Equation 5.9: Mathematical expression of the correlation between traffic volume and extension of the noise zone for the entire real data sample.

a) All Data Combined: $\quad$ Extension of Noise (in meters) $y=0.2339 \mathrm{dtv} v^{0.7273}$

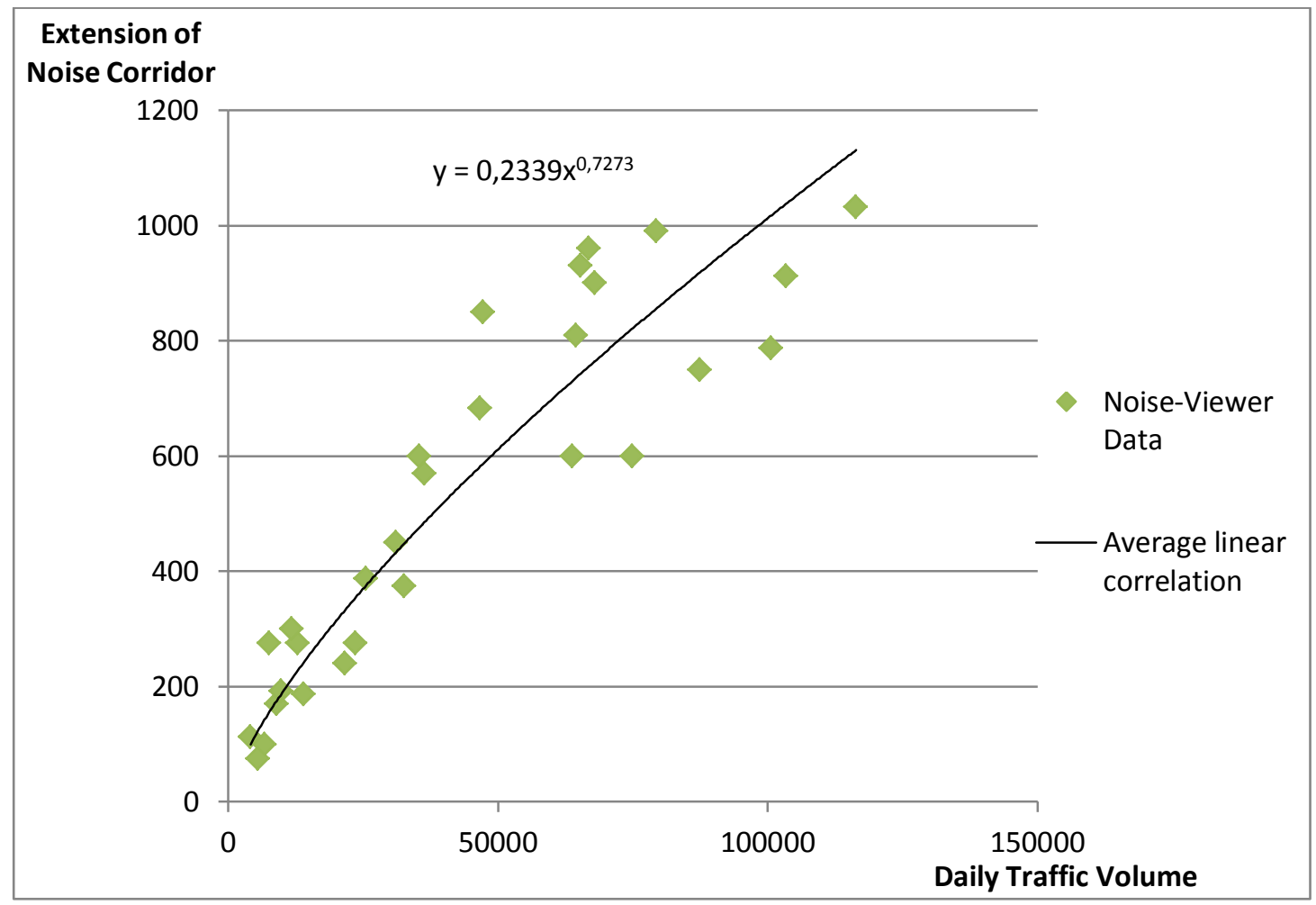

Figure 5.4: Distribution of the data sample generated from noise viewers of three German federal states.

The aggregate formula discloses spatial impact also for traffic volumes below 5,000 or 3,000 vehicles per day. Some studies suggest that noteworthy negative impacts from noise on the majority of species only begin to occur at daily traffic volumes of 5,000 to 10,000 vehicles (Forman et al. 2002, Forman et al. 2003). From their literature review, Charry and Jones (2009) conclude that 3,000 vehicles per day is the approximate threshold above which substantial ecological impacts occur. Similarly, also reviewing existing studies, Forman and Sperling (2011) state that amphibians, reptiles, mammalian carnivores, ungulates, and birds often are most threatened to die in a road at traffic levels of about 3,000 vehicles per day. For rare species with small populations like for instance the wild cat in Germany, threatening levels drop to 1,000 vehicles per day. This is confirmed by studies that state that particularly sensitive species or those, for which peak traffic volumes of overall lower use roads have an impact at the most cru- 
cial times of day, can be significantly impacted far below average thresholds of impact of for 3,000 to 5,000 vehicles per day, namely already at 1,000 vehicles per day (Parris \& Schneider 2009, Charry \& Jones 2009).

Most of the impacts other than noise, and particularly the other two most significant ones, road mortality and fragmentation, begin to impede animals and their populations at lower daily traffic volumes. While the formula developed here to quantify significantly degraded habitat adjacent to a road up to here built on noise as the spatially most extensive and farthest reaching direct impact, it at this point is viewed suitable to factor in other impacts that can amount to be of population level significance and hence affect the habitat near a road and not just a small number of individuals. Thus, it is concluded that the spatial impacts of traffic volumes lower than 5,000 vehicles per day shall also be accounted for by the developed quantitative method measuring habitat degradation.

Examining the results of the practical approach, which refers to the approach of using the entire data sample, the value for 1,000 vehicles per day is 36 meters and for 3,000 vehicles it is 79 meters. These values are considered appropriate because the further negative impacts other than noise that are present below the average minimal disturbance threshold of noise, such as microclimatic modification or the attraction of predators, can be viewed to form these values. Also the representation of the two prominent impacts of road mortality and the barrier effect, which begin to occur at daily traffic volumes of 1,000 and 3,000 vehicles per day, can be reasoned. However, the representation is very basic and it shall be emphasized that for the sakes of sensibility, understanding of the effects, and mitigation, it is probably inadequate to represent the impacts of road mortality and the barrier effect through an areal indicator. It is only admissible as a very basic account, the imperfectness of which has to be acknowledged immediately. Yet, these two impacts are not essential to reason the extension of the roadeffect zone for lower traffic volumes because the aggregate impact of pollutants, waste, noise, microclimatic modification, sedimentation, attraction of predators, and hydrological alteration, is significant enough to be suitably represented by the first areal values of lower traffic volumes, for example by 36 meters at 1,000 vehicles. It is assumed to be between 5,000 and 10,000 vehicles per day that traffic noise takes over to become the dominant negative impact with farthest reach.

The aggregate formula of the practical approach yields reliable results also for higher traffic volumes. Despite the influence of other impacts no longer being as direct as for instance in the case of changes in microclimate for areas close to a road, the further reaching effects from road mortality and the road functioning as a barrier on populations, seem to add to the degradation of habitat alone from noise to a degree that vindicates seemingly large results of road-effect zone extension for high traffic volumes. 
Compared to the mean value of the data sample at 100,000 vehicles per day, which is 1,005 meters, the value of the aggregate formula, which is 1,013 meters, is very close. Table 5.11 summarizes the expressions developed assuming either a theoretical or a practical approach. To represent the theoretical approach, the formula for freeways is used. The formula for highways can be omitted here as the difference between the two is diminutive. Under the practical approach no distinction between types of roads and their mean shares of heavy duty vehicles applies because the larger effect of heavy duty vehicles is already represented in the noise zone calculations of the data sample.

Table 5.11 indicates results for a number of exemplary traffic volumes. These results are the basis to discuss and potentially modify the resulting formulae of both approaches in the next section. The objective will be to find out if one expression is reliable to a degree that allows for it to be suggested to be added to the assessment of environmental impacts of road traffic in the future.

Table 5.11: Results of differing expressions of daily traffic volume and noise zone correlation.

\begin{tabular}{|rrrr|}
\hline $\begin{array}{c}\text { Daily traffic } \\
\text { volume }\end{array}$ & $\begin{array}{c}\text { Practical } \\
\text { approach }\end{array}$ & $\begin{array}{c}\text { Theoretical } \\
\text { approach, } \\
\text { For freeways } \\
\text { y=0.2339dtv }\end{array}$ & $\begin{array}{c}\text { Average real } \\
\text { data values }\end{array}$ \\
\hline 100 & 7 & 3 & \\
500 & 21 & 10 & \\
1,000 & 36 & 19 & \\
3,000 & 79 & 48 & \\
5,000 & 115 & 75 & 198 \\
10,000 & 190 & 136 & 264 \\
20,000 & 314 & 246 & \\
40,000 & 520 & 447 & \\
50,000 & 612 & 542 & \\
70,000 & 781 & 724 & \\
80,000 & 861 & 812 & \\
100,000 & 1,013 & 984 & \\
120,000 & 1,156 & 1,151 & \\
140,000 & 1,294 & 1,314 & \\
\hline
\end{tabular}

\subsubsection{Comparison of the two approaches pursued and development of a final methodology of quantification of impact}

Since for the theoretical approach a basic noise model was used, which was then extended to account for missing variables, the results of this approach are seen as an as 
good as possible estimation when pursuing a simplified theoretical approach. Yet, the correlation depicted by the existing data is regarded to be of higher accuracy. Nonetheless, it is viewed beneficial to test, whether the approaches can support each other or whether they suggest reconsidering assumptions and reasoning in at least one of them should resulting values be greatly dissimilar. To declare results of the expressions as similar or dissimilar strongly depends on the subjective assessment of the analyzing person. Here, results of the expression generated by the practical approach and the theoretical approach should not differ by more than 20 per cent or by more than 40 meters in order for the formulae of both approaches to be considered to be reliable. Regarding percentaged deviation, the equation of the practical approach is to be the formula of reference. Figure 5.5 depicts a visual representation of the comparison between the results of the equations of the practical approach and theoretical approach.

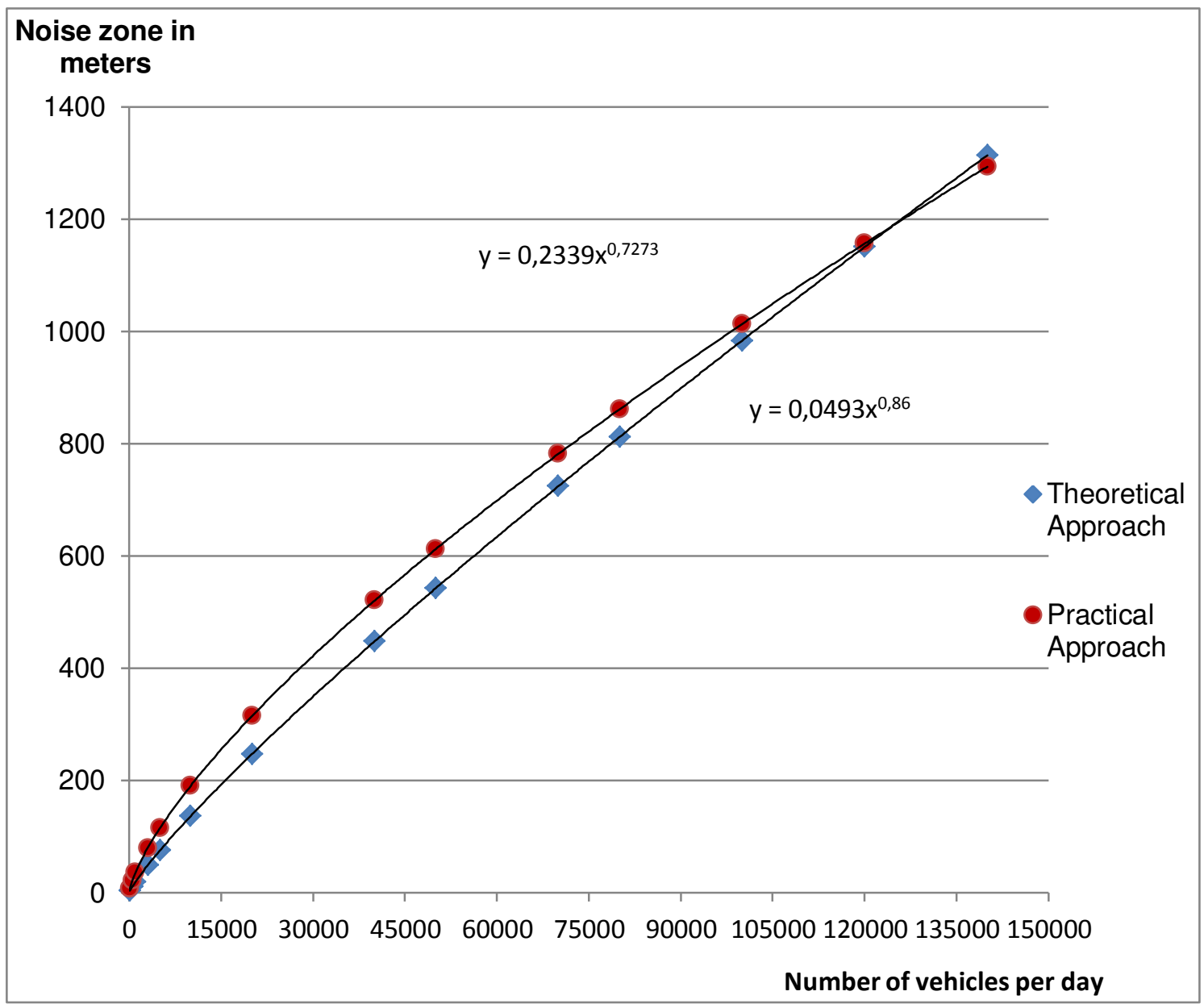

Figure 5.5: Graphical representation of the correlation between traffic volume and noise zone extension from the obtained equations.

From a first glance at the values indicated in Table 5.11 and particularly when comparing the graphs of the two formulae in Figure 5.5, the results calculated for differing traf- 
fic volumes along the potential continuum of use for the theoretical and the practical approach seem comparable. Along the continuum of use, the formula of the practical approach is slightly more concave. This shows in results for very low and very high traffic volumes to be more similar than for traffic volumes at the middle of the continuum of use, where the formula of the practical approach yields higher results. However, the difference is perceived as moderate. In the following the exact values are challenged by the rules of resemblance and reliability referred to above. For an orientation that is more exact than the one permitted by Figure 5.5, the numbers of Table 5.11 can be turned to.

While for traffic volumes lower than about 5,200 vehicles the admissible divergence of $20 \%$ between the results of the two expressions does not hold, the difference is not greater than 40 meters. From about 23,000 vehicles to 90,000 vehicles per day the difference in the results of the two approaches is larger than 40 meters, but it in turn is not larger than $20 \%$. For traffic volumes between 90,000 and 150,000 vehicles per day, both requirements are met. This leaves the range between 5,200 and 23,000 vehicles per day. Within this range both rules are violated. The largest deviation occurs at about 10,000 vehicles where results of the theoretical approach are about 28 per cent or 54 meters smaller than those of the practical approach. While ex ante this deviation was defined to require reconsideration and remodeling of one or of both of the formulae, the following three reasons argue in favor of accepting the overall more accurate formula of the practical approach as it is: firstly, the data sample of the practical approach contains no values of very low traffic volumes; the lowest data pair had a daily traffic volume of 4,109 vehicles. This means that this range is slightly underrepresented and the reliability of the formula in this range is likely not to be as accurate. Further data pairs of this range could not be found because they apply to smaller roads than the ones represented by the noise viewers. Secondly, regarding the theoretical approach, several assumptions went into the simplified theoretical modeling. While this means that the theoretically derived formula can potentially be improved, the necessary effort is perceived impracticable and would not justify the potential gains because the practical approach overall is viewed more reliable. Thirdly and most importantly, the potentially slightly too high results of the extension of the noise zone from the practical approach are considered to be of use in order rudimentarily to represent the spatial impacts of other impacts of traffic referred to in section 5.1.2.6 like emissions, microclimatic modification, sedimentation, attraction of predators, or hydrological alteration that are estimated to dominate the spatial impacts of noise at low traffic volumes up to about 8,000 vehicles per day.

Overall, as the difference at the beginning of the continuum of use is viewed tolerable and as the remainder of the resulting values are relatively similar, it is concluded that 
the formula of the practical approach stands the comparison to the expression of the theoretical approach and that its equation reliably represents the correlation between traffic volume and the width of a noise-dominated road-effect zone.

That the values of the expression of the theoretical approach were calculated assuming an average share of heavy duty vehicles for freeways of $14.56 \%$ does not render the comparison useless for federal highways because as shown in Table 5.9, the absolute values of the marginally differing formulae differ very little. The similarity of the results for dissimilar shares of heavy duty vehicles under the two expressions of the theoretical approach (see Equations 5.7d and e) suggests that the weakness of the practical approach not to account for dissimilar shares of heavy duty vehicles is tolerable. This is despite vehicle size being a major variable for the amount of noise being produced by a moving vehicle (see Table 5.15).

Despite the conclusion that results of the equations of the two approaches are similar enough to strengthen rather than to question the reliability of either approach, a notable difference in the results is present. For the majority of the continuum of use, namely for all traffic volumes below 120,000 dtv, the practical approach yields values that are significantly higher than those of the theoretical approach (see Table 5.11 and Figure 5.5).

Three potential reasons for this difference were mentioned before, they are: firstly, the data sample consists of values which were calculated using the $L_{D E N}$ formula. In the calculation of the average sound pressure level for 24 hours using the $L_{D E N}$ formula (Equation 5.6), the impact of evening and night-time noise are artificially increased in order to account for the aggravated impact of noise on humans during those times of day. In section 5.1.2.3 it was decided that too little is known on the severity of impacts on fauna at different times of day and that too little discussion on the topic of how humans want to affect non-human nature overall, and particularly with regard to noise, has happened, for instance because dominant anthropocentric, utilitarian thinking to some extent conflicts with the seemingly unconditional but still unclear goal of protecting biological diversity. Therefore the expression developed pursuing a theoretical approach was chosen to calculate purely energetic values, meaning that the impact of nighttime noise is not artificially upvalued. For this reason the values of the theoretical approach were expected to be lower than the expression generated from the data sample. This argumentation holds also because the precautionary principle is assumed to be sufficiently implemented in choosing a sound pressure level value of $55 \mathrm{~dB}(\mathrm{~A})$ as a rather low threshold of animal disturbance and in assuming a rather low total attenuation-value of $3.5 \mathrm{~dB}(\mathrm{~A})$ per doubling of distance. Under the practical approach, the gathering of data in mostly open landscapes and the setting of the disturbance threshold of sound pressure level at $55 \mathrm{~dB}(\mathrm{~A})$ are regarded as decisions to apply the precau- 
tionary principle sufficiently. Therefore, it is decided to correct the practical approach for being biased by the overestimation of noise inherent to $L_{D E N}$. Calculating $L_{D E N}$ and ' $L_{D E N-e n e r g e t i c}$ ', $L_{D E N-e n e r g e t i c}$ being the average sound pressure level for 24 hours free of a weighting of daytimes, for various daily traffic volumes between 10,000 and 100,000 vehicles, which is assumed to be the foremost continuum of use of federal highways and freeways, showed that $L_{D E N}$ values were between 5.0 and $5.8 \%$ higher than those of $\mathrm{L}_{\mathrm{DEN} \text {-energetic. }}$ Applying a correction of an average of $5.4 \%$ to the practical approach changes the equation to the one indicated in Table 5.12.

Table 5.12: Noise zone expressions derived from the data sample changed to $L_{D E N-\text { energetic. }}$

\begin{tabular}{|l|c|}
\hline $\begin{array}{c}\text { Type of 24- } \\
\text { hour method }\end{array}$ & $\begin{array}{c}\text { Practical } \\
\text { approach }\end{array}$ \\
\hline$L_{D E N}$ & $y=0.2339 \mathrm{dtv}^{0.7273}$ \\
$\mathrm{~L}_{\mathrm{DEN} \text {-energetic }}$ & $\mathrm{y}=0.2212 \mathrm{dtv}^{0.7273}$ \\
\hline
\end{tabular}

The modification of the formula has an impact on the values of noise zone width of the practical approach. The change in results is indicated in Table 5.13.

Table 5.13: Display of the noise zone results using $L_{D E N-e n e r g e t i c}$ for the practical approach.

\begin{tabular}{|rccc|}
\hline $\begin{array}{c}\text { Daily } \\
\text { traffic } \\
\text { volume }\end{array}$ & $\begin{array}{c}\text { Practical } \\
\text { approach } \\
L_{\text {DEN }}\end{array}$ & $\begin{array}{c}\text { Practical } \\
\text { approach } \\
\mathbf{L}_{\text {DEN-energetic }}\end{array}$ & $\begin{array}{c}\text { Theoretical } \\
\text { approach }\end{array}$ \\
\hline 100 & $y=0.2339$ dtv $^{0.7273}$ & $y=0.2212$ dtv $^{0.7273}$ & $y=0,0493$ dtv $^{0.86}$ \\
500 & 7 & 6 & 3 \\
1,000 & 21 & 20 & 10 \\
3,000 & 36 & 34 & 19 \\
5,000 & 79 & 75 & 48 \\
10,000 & 115 & 108 & 75 \\
20,000 & 314 & 179 & 136 \\
40,000 & 520 & 297 & 246 \\
50,000 & 612 & 492 & 447 \\
70,000 & 781 & 579 & 542 \\
80,000 & 861 & 739 & 724 \\
100,000 & 1,013 & 814 & 812 \\
120,000 & 1,156 & 958 & 984 \\
140,000 & 1,294 & 1,094 & 1,151 \\
\hline
\end{tabular}


With regard to the comparison between the theoretical approach and the practical approach, the power function of the modified expression of the practical approach resembles the theoretical approach more than when using $L_{D E N}$ data. While it was expected that the extent of difference of higher results of the practical over the theoretical approach would diminish, it could not be foreseen that the equations and results would resemble more. However, this strengthens the reliability of both approaches because despite the rules of resemblance and reliability of a discrepancy in noise zone results at a certain traffic level not to surpass either 40 meters or 20 per cent are still violated for a significant scope of traffic volumes, the scope of the violation more than halved now ranging from 7,900 to 15,300 vehicles per day instead of prior ranging from 5,200 to 23,000 vehicles per day. The stronger resemblance becomes visually evident in Figure 5.6 and also in comparison of Figures 5.5 and 5.6.

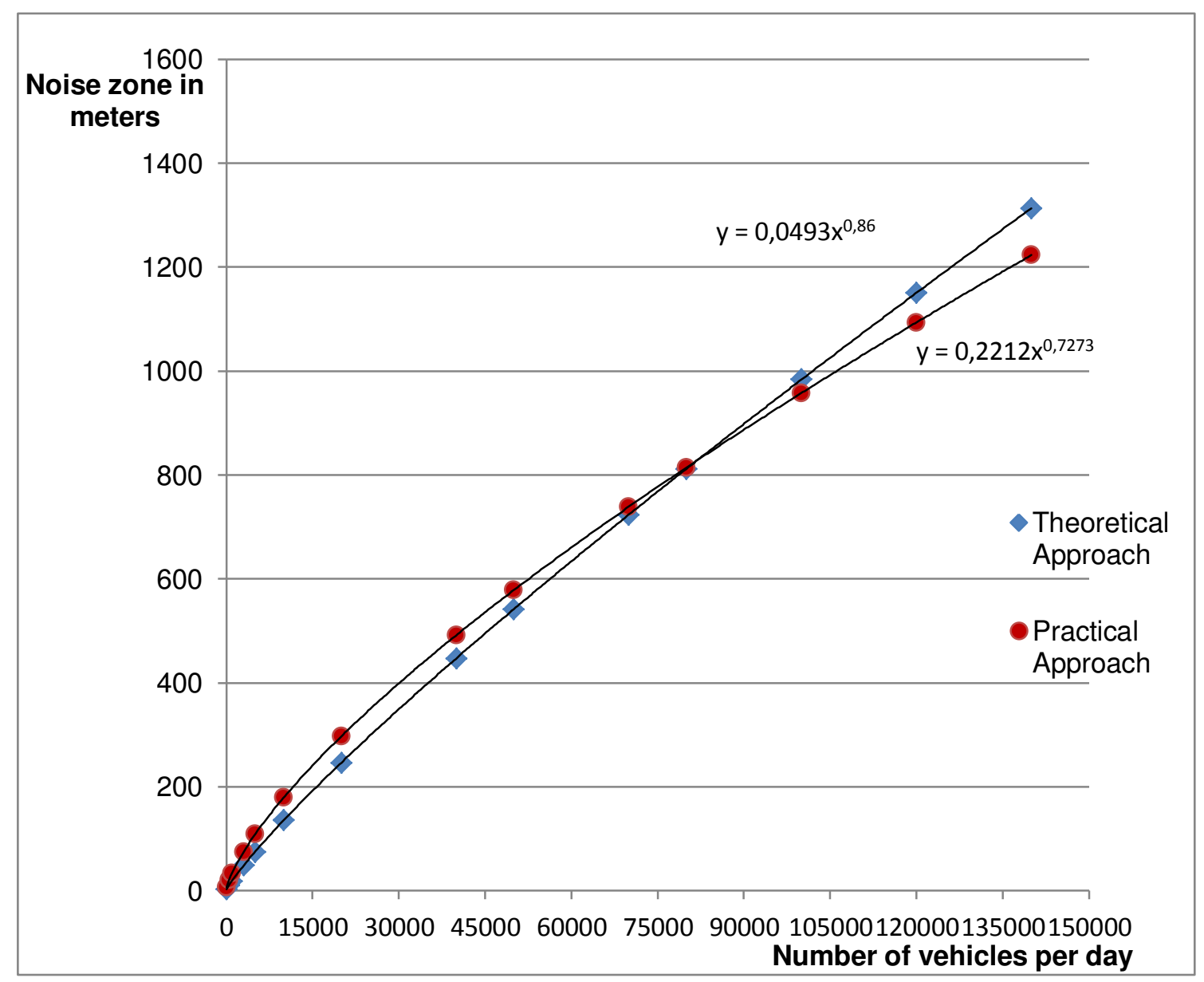

Figure 5.6: Graphical representation of the correlation between traffic volume and noise zone extension when adjusting the practical approach to $L_{D E N-e n e r g e t i c}$.

The second and third potential reason, why the practical approach originally yielded higher results than the theoretical one do not seem to apply because although the ad- 
justed equation of the practical approach still yields higher results from the beginning of the continuum of use up to about 82,000 vehicles, from this level onwards the theoretical approach yields higher values so that it cannot be said that the practical approach yields higher values overall. Yet, the other two potential reasons to produce a difference in results are mentioned. It is now helpful that they are related to one another and seem to level each other. On the one hand, data of the practical approach was gathered for open landscapes because they represent the most frequent condition and because data of comparable conditions was needed better to test for the correlation between traffic volume and noise zone extension. While slightly overestimating the size of the spatial impact on the landscape was viewed tolerable due to the assumed small size of the misrepresentation and the application of the precautionary principle, this could have led to obtaining higher values than the theoretical approach. On the other hand, it seems that the third potential reason for differing results, which is the drop-off in sound pressure level for every doubling of distance in being $3.5 \mathrm{~dB}(\mathrm{~A})$ was selected suitably in order to represent conditions of attenuation similar to those in open landscapes. Had the value been too large, results of the expression of the theoretical approach would have been significantly smaller than the ones of the practical approach.

In conclusion, after adjusting the practical approach to $L_{D E N-e n e r g e t i c}$, the results of the expressions of the practical approach and the theoretical approach do not differ by much (see Table 5.13 or Figure 5.6). It stands as a conclusion of the comparison and analysis of the approaches that the expression obtained under the practical approach, after being adjusted to represent purely energetic sound pressure levels, is of sufficiently high accuracy and quality to allow for an approximate quantification of some of the spatial ecological impacts that are produced by traffic and that occur adjacent to a road, particularly of the impact of noise. Equation 5.10 repeats the formula. In order to use the expression in corporate environmental management, in section 5.1.2.9 the formula is extended not to yield the aggregate impact of a road with a certain traffic volume, but to yield the impact of an individual user.

Equation 5.10: Final formula to calculate the average width of a noise-dominated road-effect zone.

Extension of Noise (in meters) $y=0.2212 d t v^{0.7273}$

\subsubsection{Comparison of the results of the formula developed in this work to other results}

In a last step of analyzing and validating the theoretically derived formula and the final expression obtained from the data sample, their assumptions and results are compared 
to other studies that are related to the topic. However, as mentioned above, most of the few studies that were found to deal with the topic either refer to particular species and sites (Reijnen \& Foppen 2006, Helldin et al. 2013) or to aggregate national scales (Forman 2000). Only one study published by Dooling and Popper in 2007 in its complementary material reports on the direct correlation between daily traffic volume and noise levels at varying distances from a road. The graphic of concern, which is entitled 'Typical highway noise levels', displays results yielded by the Federal Highway Administration Noise Model (TNM) of the United States of America. The graphic (see Figure 5.7) reports sound pressure level extension subject to hourly daily traffic volume. The comparison of the results to those obtained using the expressions developed above is majorly hampered because too little information on the noise model, its assumptions, and the conversion rate between hourly and daily traffic volume, could be attained. Most likely the hourly traffic volumes of the graphic stem from a specific project. This makes the extrapolation to average daily traffic volumes difficult. While in the USA federal regulations (Procedures for Abatement of Highway Traffic Noise and Construction Noise - 23 CFR 772) require the use of the worst case noise hour when calculating existing and predicting future noise zone extension, in Germany the $30^{\text {th }}$ worst hour of a year is used as the 'applicable hourly traffic volume' (MSV = maßgebliche stündliche Verkehrsstärke). In a national assessment in Germany, the percentage of the $30^{\text {th }}$ worst noise or busiest road hour ranged to represent between 7 and $20 \%$ of the total quantity of average daily traffic volume. This large spectrum explains because the ratio depends highly on the function of a road, which differs between meeting diverse user demands like commuting to work, driving into a city, or going on vacation. If an overall average had to be yielded, data indicates that the $30^{\text {th }}$ worst noise hour corresponds to 10 to $11 \%$ of daily traffic volume (Fitschen \& Nordmann 2012). While no data is known for the USA, at the Federal Highway Administration the average share of the worst noise hour of a 24 hour period is assumed to be around 10\% (Alexander 2013). Considering the stricter definition of the worst noise hour in the US when compared to Germany, this number seems too low and it might be considerably higher for any specific road. The attempt to extrapolate daily traffic volumes from the hourly traffic volumes (htv) indicated by Dooling and Popper (2007) yields largely differing results depending on whether an extrapolating factor for the worst noise hour of either 10 or $15 \%$ is assumed. For instance, for 6,000 vehicles per hour the daily value could be between 40,000 and 60,000 vehicles. Due to this uncertainty, in the graphical representation of the results of the US traffic noise model, a conversion to daily traffic volumes is forgone and the results correspond to hourly traffic volumes. Despite this limitation for comparison, one clear distinction to the most important assumption made under the theoretical approach in this work is apparent. This is the used value of attenuation per doubling of distance. From Figure 5.7 it can be read that under the US traffic noise model, with 
every doubling of distance, sound pressure levels fall between 6 and $7 \mathrm{~dB}(A)$. For instance for the graph that describes the spreading of noise at 3,000 vehicles per hour, sound pressure level falls from 61.5 to $55 \mathrm{~dB}(\mathrm{~A})$ when going from 61 to 122 meters of distance or from 63 to $56 \mathrm{~dB}(\mathrm{~A})$ at 6,000 vehicles per hour when moving out from 122 to 244 meters. This magnitude of attenuation is twice the value assumed under the theoretical approach pursued above.

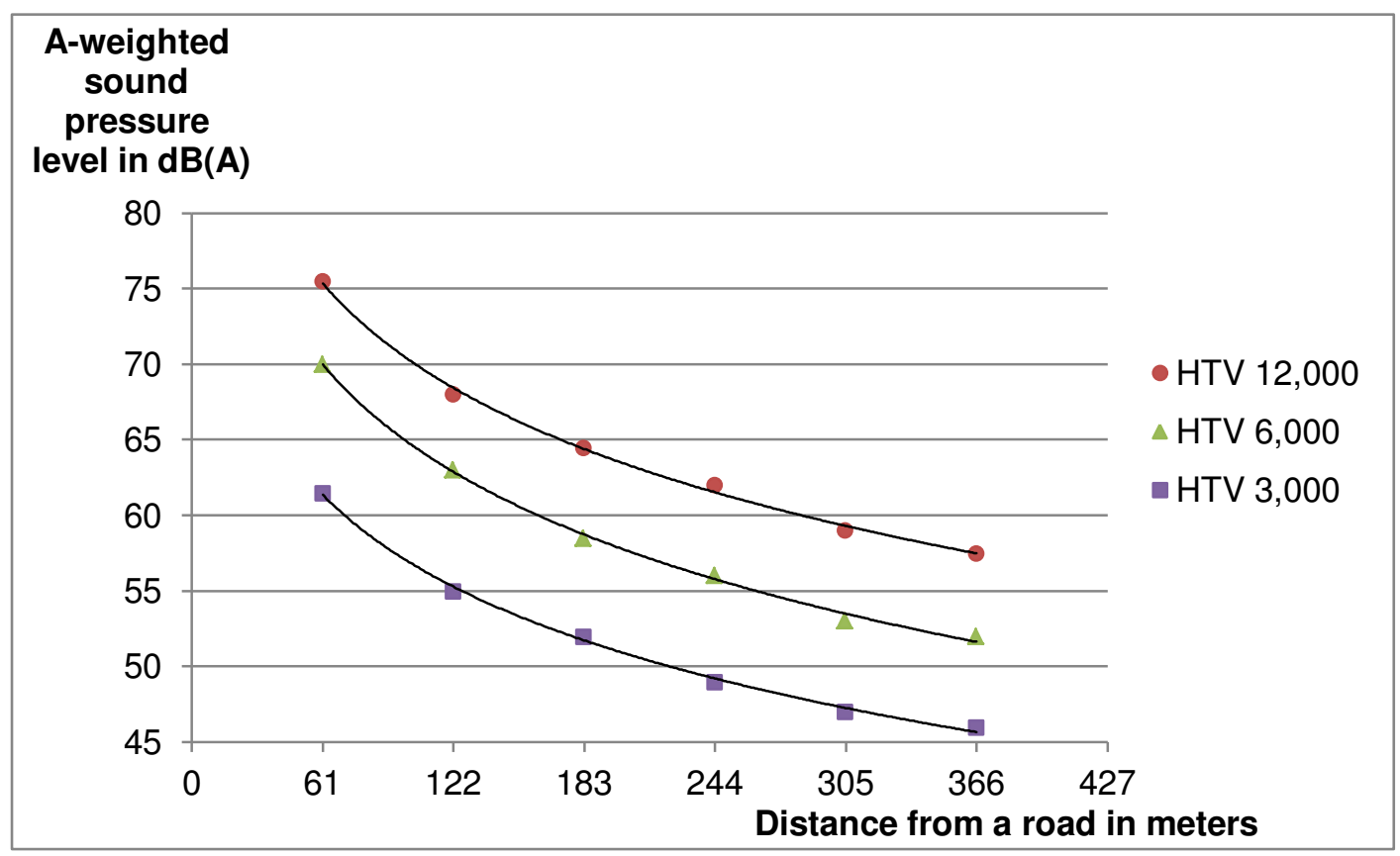

Figure 5.7: Noise levels and their extensions at peaking hourly traffic volumes; adapted from Dooling and Popper 2007.

While a comparison of the results indicated in the graphic to the values yielded by the final expression of the practical approach is imprecise, one exemplary estimate shall be given. For a quantity of 6,000 vehicles per busiest hour, the expansion of sound pressure levels $\geq 55 \mathrm{~dB}(\mathrm{~A})$ in the graphic is about 268 meters, whereas it is between 425 and 615 meters under the final expression of the practical approach when assuming respective daily traffic volumes between 40,000 and 60,000 vehicles. This difference can also be seen when plotting the average real data values of this work in a way similar to the US data as done in Figure 5.8. Comparing Figures 5.7 and 5.8, the slopes of the curves that represent the German noise expansion sample are much weaker compared to the steeper decline of curves representing the US data. For Germany, the progression of the curves shows that per doubling of distance $\mathrm{dB}(\mathrm{A})$-values decrease only by 3.4 to 3.5 points. In order to account for possible sensivities, two scenarios, one in an open another in a cleft landscape, are pictured in Figure 5.8 that represents the German data. As the two curves do not deviate dramatically, the composition of the landscape is unlikely to explain the greatly different drop-off rates. 


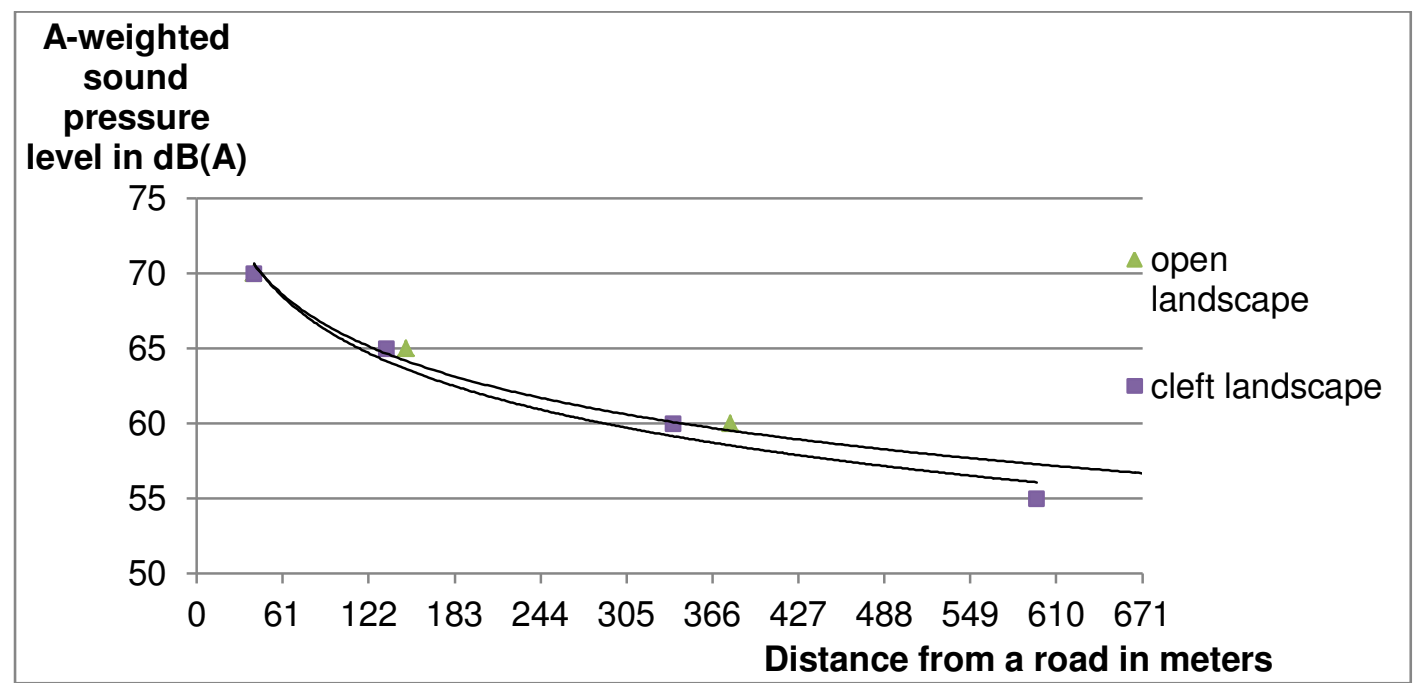

Figure 5.8: Noise levels and their extension as under the $L_{D E N-e n e r g e t i c} S$ Scenario for a German freeway at 64,000 vehicles per day.

Provoked by the dissimilar level of attenuation of the US traffic noise model and in order again to test the reliability of the expression of the theoretical approach developed above, the theoretical approach was followed again, this time assuming first a 6 and then a $4 \mathrm{~dB}(\mathrm{~A})$ decrease in sound pressure per doubling of distance. The resulting values are indicated in Table 5.14. The results for a $6 \mathrm{~dB}(\mathrm{~A})$ attenuation are much smaller than those of the original $3.5 \mathrm{~dB}(\mathrm{~A})$ drop-off rate and the $4 \mathrm{~dB}(\mathrm{~A})$ drop-off rate from 10,000 vehicles per day onwards. Compared to the average values of the data sample (Table 5.14, column 5) it becomes evident that assuming a $6 \mathrm{~dB}(\mathrm{~A})$ decrease per doubling of distance for the noise calculation formulae used in Germany is not appropriate.

Next, it was tested, how an only slightly higher attenuation value of $4 \mathrm{~dB}(\mathrm{~A})$ would play out. Despite yielding more realistic results, particularly for traffic volumes larger 50,000 vehicles per day, the values also differ significantly from those of the data set (see Table 5.14 , columns $2 \& 3$ ). It is concluded that due to the quality of the existing data and due to the similar expansion of impact zones determined in studies on birds by Reijnen et al. (1996) and Forman et al. (2002) (see section 5.1.2.5) the firstly assumed attenuation value of $3.5 \mathrm{~dB}(\mathrm{~A})$ is kept. The approximate decrease of noise levels of the data sample plotted in Figure 5.8 being 3.4-3.5 dB(A) supports this decision. Besides, too little is known on the methodology and the circumjacent assumptions made in the US noise model. Further doubts about the accuracy of the results of Figure 5.7 and the indicated $\mathrm{dB}(\mathrm{A})$-drop-off rate to be between 6 and $7 \mathrm{~dB}(\mathrm{~A})$ arise from within the very same publication where Dooling and Popper (2007) state that ground and atmospheric absorption together can increase geometric attenuation to 5-6 $\mathrm{dB}(\mathrm{A})$ per doubling of distance. Even this more favorable scenario of attenuation does not match the drop-off rate of the graphic. Drop-off values as of Figure 5.7 seem realistic only if very effective 
shielding by natural or human-made features is present or if the height of the source and the receiver are chosen rather adversely to the spreading of sound pressure. However, both of these conditions cannot suitably be assumed for the impact of noise on animals at the landscape level. Therefore, the energetically adjusted expression of the practical approach prevails as the most accurate and reliable formula to determine the average noise zone extension of a road to be deduced from traffic volumes.

Table 5.14: Expansion of the noise zone assuming total attenuation values of $3.5,4$ and $6 \mathrm{~dB}(\mathrm{~A})$ per doubling of distance.

\begin{tabular}{|c|c|c|c|c|}
\hline $\begin{array}{c}\text { Daily } \\
\text { traffic } \\
\text { volume }\end{array}$ & $\begin{array}{c}\text { Extension of noise } \\
\text { zone; attenuation } 3.5 \\
\mathrm{~dB}(\mathrm{~A}) \\
\mathrm{y}=0.0493 \cdot \mathrm{dtv}^{0.86}\end{array}$ & $\begin{array}{c}\text { Extension of noise } \\
\text { zone; attenuation } 4 \\
\mathbf{d B}(\mathbf{A}) \\
\mathrm{y}=0.1074 \cdot \mathrm{dtv}^{0.7526}\end{array}$ & $\begin{array}{c}\text { Extension of noise } \\
\text { zone; attenuation } 6 \\
\qquad \begin{array}{c}\mathbf{d B}(\mathrm{A}) \\
\mathrm{y}=0.6607 \cdot \mathrm{dtv}^{0.5017}\end{array}\end{array}$ & $\begin{array}{c}\text { Average } \\
\text { real data } \\
\text { values }\end{array}$ \\
\hline 100 & 3 & 3 & 7 & \\
\hline 500 & 10 & 12 & 15 & \\
\hline 1,000 & 19 & 19 & 21 & \\
\hline 3,000 & 48 & 44 & 37 & \\
\hline 5,000 & 75 & 65 & 47 & 96 \\
\hline 10,000 & 136 & 110 & 67 & 198 \\
\hline 20,000 & 246 & 185 & 95 & 264 \\
\hline 40,000 & 447 & 312 & 135 & \\
\hline 50,000 & 542 & 369 & 150 & 690 \\
\hline 70,000 & 724 & 476 & 178 & \\
\hline 80,000 & 812 & 526 & 190 & \\
\hline 100,000 & 984 & 622 & 213 & 1,005 \\
\hline 120,000 & 1,151 & 714 & 233 & \\
\hline 140,000 & 1,314 & 802 & 252 & \\
\hline
\end{tabular}

\subsubsection{Further development of the interim method}

As of the state of the formula as indicated in Equation 5.10, three shortcomings remain: firstly, the assumption of a noise disturbance threshold at $55 \mathrm{~dB}(\mathrm{~A})$ because the threshold only is an educated estimate as it varies per species and per time of the day and has not been sufficiently discussed in science and society, secondly, the dominance of the impact of noise over other impacts of expanding spatial effect like genetic depletion due to mortality or the barrier function, and thirdly, the inability to consider relevant parameters like topography, speed, or vehicle size individually. Yet, with regard to the overall objective, which was to inspire and contribute to more complete environmental management efforts for road transport, all three shortcomings are viewed tolerable as 
long as they are communicated clearly to potential users and to those who wish to criticize and further develop the indicator.

While the first two shortcomings were attended to in this work and here cannot be improved further, the third weakness of the equation at large is solved in the following paragraphs. Regarding the first weakness, more studies on impacts on animals and discussions on admissible degrees of effect and risk in society are necessary. Besides, knowledge of the 'perfect' threshold is not essential in order to begin to reducing an overall clearly adverse impact. The second weakness is regarded to be tolerable as long as the dominance of noise in determining spatial impact is highlighted and not sufficiently accounted for impacts are stated. Besides, it seems impractical to unite all effects in one indicator. Additional methods of assessment of other spatial impacts can be added to the one dominated by noise if regarded necessary. Moreover, the above formula can account for some of the other spatial ecological impacts like change of microclimate, wastes, increased $\mathrm{CO}_{2}$ composition, etc. For further reaching and less direct impacts that occur at the population level, such as mortality, genetic depletion, or the reduced richness, stability, and functioning of local ecosystems, additional measures that allow for better informed decision-making and improved environmental management may be necessary.

Regarding the third weakness, not to account for topography individually is viewed admissible because freight transports usually cover large distances so that average landscape values can be used. However, the consideration of vehicle size is of significance, because for a fair calculation of impact per user, vehicle size has to be accounted for. While in the expression of the theoretical approach it can be differentiated between heavy duty vehicles and all other vehicles, also the final expression derived from the data sample can be extended to calculate impact in accordance with vehicle size. Here, two possibilities arise: Firstly, to differentiate between heavy duty and nonheavy duty vehicles (HDVs), or secondly, to differentiate between all major vehicle groups, i.e. cars, buses, motorcycles, trucks, etc. In the following, the difference in individual share of degraded area when differentiating between HDVs and non-HDVs is shown. Assuming a traffic volume of 50,000 vehicles and multiplying the corresponding areal value by two in order to account for each side of the road, and again to multiply the result by the length of a section $\mathrm{i}$, which in this example is assumed to be 20 kilometers, further assuming a share of heavy duty vehicles of $15 \%$ as well as an impact ratio for heavy duty vehicles to non-heavy duty vehicles of $3: 1$, yields the share of noise driven landscape impact (NDLI) for one heavy duty vehicle within one year calculated in Equation 5.11b. In Equation 5.11a the resulting value when not differentiating between vehicle types is calculated. 
Equations 5.11: Calculation of noise driven landscape impact not differentiating (a) and differentiating (b) between HDVs and non-HDVs.

a) Noise Driven Landscape Impact of $1 \mathrm{HDV}$ when not differentiating by vehicle type $=$ $\frac{\text { noise zone in meters }\left(0,2212 \cdot d t v_{i}{ }^{0.7273}\right) \cdot 2 \cdot \text { length of section } \mathrm{i} \text { in meters }}{d t v_{i} \cdot \text { days of the year }}$

Hence:

$$
\begin{aligned}
& \text { NDLI of } 1 \mathrm{HDV}=\frac{\left(0,2212 \cdot 50,000^{0.7273}\right) \mathrm{m} \cdot 2 \cdot 20,000 \mathrm{~m}}{50,000 \cdot 365} \\
\Leftrightarrow \quad \text { NDLI of } 1 \mathrm{HDV} & =\frac{24,470,800 \mathrm{~m}^{2}}{50,000 \cdot 365} \\
\Leftrightarrow \quad \text { NDLI of } 1 \mathrm{HDV} & =1.3409 \mathrm{~m}^{2}
\end{aligned}
$$

b) Noise Driven Landscape Impact of $1 \mathrm{HDV}$ when differentiating by vehicle type $=$ $\frac{\text { noise zone in meters }\left(0,2212 \cdot d t v_{i}^{0.7273}\right) \cdot 2 \cdot \text { length of section i in meters }}{\left[\left(d t v_{i} \cdot \text { share of non-hdv }{ }_{i} \cdot \mathrm{f}\left(\mathrm{v}_{\text {non-hdv }}\right)\right)+\left(d t v_{i} \cdot \text { share of } \mathrm{hdv}_{i} \cdot \mathrm{f}\left(\mathrm{v}_{\mathrm{hdv}}\right)\right)\right] \cdot \text { days of the year }} \cdot \mathrm{f}\left(\mathrm{v}_{\mathrm{hdv}}\right)$

Hence:

$$
\begin{aligned}
\text { NDLI of } 1 \mathrm{HDV} & =\frac{\left(0,2212 \cdot 50,000^{0.7273}\right) \mathrm{m} \cdot 2 \cdot 20,000 \mathrm{~m}}{(50,000 \cdot 0.85 \cdot 1+50,000 \cdot 0.15 \cdot 3) \cdot 365} \cdot 3 \\
\Leftrightarrow \quad \text { NDLI of } 1 \mathrm{HDV} & =\frac{24,470,800 \mathrm{~m}^{2}}{65,000 \cdot 365} \cdot 3 \\
\Leftrightarrow \quad \text { NDLI of } 1 \mathrm{HDV} & =3.0943 \mathrm{~m}^{2}
\end{aligned}
$$

As reasoned above, it is considered appropriate to split the spatial ecological impact among all users within one year because the potential effects of altered usage within one year are viewed to affect future infrastructure planning decisions. In addition, yearly results are suitable for companies because their principal reporting and management periods usually are one year. Comparing the result of $3.0943 \mathrm{~m}^{2}$ to a value of 1.3409 $\mathrm{m}^{2}$, when not differentiating by vehicle size, shows that a distinction is relevant for a fair allocation of impact.

The significance of the difference evokes pursuing the second possibility of allocation, which is to distinguish in more detail between all major vehicle groups as done in section 5.1.1. Since no average shares of all vehicle groups on roads are reported, again shares of the total national mileage in billion ton-kilometers are used (Table 5.15). Impact factors per vehicle type are expressed as passenger car units (PCUs). For the 
generation of sound pressure level, the PCUs valid for the areal usage and the material demand of dissimilar vehicle types that were relevant in section 5.1.1 cannot be used identically. Although they include most of the variables relevant for the generation of sound pressure levels, they do not set special emphasis on the generation of noise. At speeds greater $50 \mathrm{~km} / \mathrm{h}$ tire noise becomes the dominant noise source of individual vehicles. While engine noise is the most relevant source of noise at speeds lower $50 \mathrm{~km} / \mathrm{h}$, at higher speeds velocity itself becomes a key variable in the generation of noise (ODOT 2006). Bartolomaeus (2010) analyzed the road noise emission models used in Europe, which are the RLS-90 in Germany, the RVS 04.02.11 in Austria, NMPB 2008 in France, SonRoad in Switzerland, and CNOSSOS in the entire EU. The models use different methodologies. RLS-90 and RVS use pure A-weighted methods, NMPB 2008 and SonRoad use the standard spectrum, and CNOSSOS uses differentiated spectral data of acoustic emissions. While the methods differ in the input data considered like rolling and engine noise, gradient, surface, traffic volume, and speed, as well as in the way of their inclusion, results are comparable. When relating the noise emissions of passenger cars and heavy duty vehicles, only at speeds below $50 \mathrm{~km} / \mathrm{h}$ do the results of the models, and particularly of RLS and the RVS, deviate significantly. Apart from RLS, from 50 to $100 \mathrm{~km} / \mathrm{h}$ the other four models yield similar ratios of the generation of sound pressure level of cars and trucks. In Figure 5.9 it is illustrated how many passenger cars equal the noise emission of one heavy duty vehicle at an identical speed of both vehicles. At a vehicle speed of $80 \mathrm{~km} / \mathrm{h}$ one heavy duty vehicle produces the noise emissions of between 3 and 9.5 passenger cars. While CNOSSOS is the model of highest complexity yielding a ratio of 1 truck to 3 cars, NMPB 2008 excels for the quality of input data suggesting a ratio of 1 to 7 (Bartolomaeus 2010 \& 2013).

There is one slight difficulty with these ratios, which is that passenger cars and heavy duty vehicles in reality do not move at the same speed, but that passenger cars particularly on freeways tend to move faster than heavy duty vehicles. Despite no data on average speed per vehicle type per road type is available, in Germany on freeways an average speed of heavy duty vehicles between 70 and $80 \mathrm{~km} / \mathrm{h}$ and of passenger cars between 90 and $110 \mathrm{~km} / \mathrm{h}$, including traffic jams and lower speed limits like in road works, is assumed. The question is: what effects do dissimilar average speeds of vehicle types have on the average generation of sound pressure and on the ratios referred to above and indicated in Figure 5.9? Here, the road noise emission model of Germany will be used to approximate the change in sound pressure level when the average speed of passenger cars is increased by $20 \mathrm{~km} / \mathrm{h}$ (see Equations 5.4 ). $20 \mathrm{~km} / \mathrm{h}$ is the estimated difference of average speeds of passenger cars and heavy duty vehicles. In the RLS-90, for the standard calculation of sound pressure levels of freeways, vehicle speeds or speed limits of heavy duty vehicles of $80 \mathrm{~km} / \mathrm{h}$ and of passenger cars of 100 
$\mathrm{km} / \mathrm{h}$ are assumed. In addition, the model consists of formulae, which allow calculating noise emissions for conditions where input variables differ from the assumed standard. Among them are formulae for deviating vehicle speeds $\left(D_{s}\right.$, Equation 5.12a). That $D_{s}$ in the model is defined as abbreviating speed limit is not relevant for the calculation of the quantity of increase of noise emissions when increasing speed by $20 \mathrm{~km} / \mathrm{h}$. All necessary formulae are indicated in Equations 5.12.

Equation 5.12: Formulae used under RLS-90 to account for speed limits that deviate from the assumed standards (source: VBUS 2006).
a) $D_{s}=L_{c a r}-37.3+10 \cdot \log \frac{100+\left(10^{\frac{D}{10}}-1\right) \cdot p}{100+8.23 \cdot p}$
b) $L_{c a r}=27.7+10 \cdot \log \left[1+\left(0.02 \cdot v_{c a r}\right)^{3}\right]$
c) $L_{\text {truck }}=23.1+12.5 \cdot \log \left(v_{\text {truck }}\right)$
d) $D=L_{\text {truck }}-L_{\text {car }}$

$D_{s} \quad=$ size of deviation of differing speed limits or speeds in $\mathrm{dB}(\mathrm{A})$

$\mathrm{L}_{\mathrm{car}} \quad=$ time-average sound pressure level per passenger car per hour

$\mathrm{L}_{\text {truck }} \quad=$ time-average sound pressure level per heavy duty vehicle per hour

$\mathrm{D}=$ differential gauge

$\mathrm{v}_{\mathrm{car}} \quad=$ assumed speed limit or speeds for passenger cars

$\mathrm{v}_{\text {truck }} \quad=$ assumed speed limit or speeds for heavy duty vehicles

$\mathrm{p} \quad=$ share of heavy duty vehicles

The three input variables that need to be known in order to calculate the size of abbreviation of noise emissions in $\mathrm{dB}(\mathrm{A})$ of non-standard speed limits are the speed limits or speeds for passenger cars and heavy duty vehicles and the share of heavy duty vehicles. Irrespective of the value of $p$, when choosing $v_{\text {car }}$ to be $100 \mathrm{~km} / \mathrm{h}$ and $v_{\text {truck }}$ to be $80 \mathrm{~km} / \mathrm{h}, D_{\mathrm{s}}$ is zero. When modifying $v_{\text {car }}$ to be $120 \mathrm{~km} / \mathrm{h}$ while leaving $v_{\text {truck }}$ at $80 \mathrm{~km} / \mathrm{h}$ and assuming a share of heavy duty vehicles of $17 \%$, noise emissions rise by 2.083 $\mathrm{dB}(\mathrm{A})$. In the calculation the share of heavy duty vehicles is a factor of marginal impact as assuming a value of $p$ of 10 instead of $17 \%$ produces a rise in noise emissions to $2.094 \mathrm{~dB}(\mathrm{~A})$, which is an insignificant difference compared to $2.083 \mathrm{~dB}(\mathrm{~A})$.

As the basic noise propagation model (Equation 5.4a) in a situation of daily traffic volume of 42,000 vehicles and the share of heavy duty vehicles being $17 \%$ yields a 24 hour energetic noise emission average of about $66 \mathrm{~dB}(\mathrm{~A})$, it can be seen that while for the absolute spreading of noise an increase in $2.083 \mathrm{~dB}(\mathrm{~A})$ is meaningful, for a fair allocation of impact a change in vehicle speeds at higher velocity does not make a very large difference. This is very different at lower speeds, where an increase in speed has a much higher consequence on resulting $\mathrm{dB}(\mathrm{A})$-values. Overall, it is concluded that the 
ratio of sound pressure levels of passenger cars and heavy duty vehicles as indicated in Figure 5.9 is appropriate to be used to describe the generation of sound pressure by vehicle type because an increase in an assumed average vehicle speed of passenger cars does not gravely affect, that is decrease, the ratio of noise emissions of trucks and cars at high vehicle speeds relevant on mostly larger roads and depicted in Figure 5.9. However, a slight correction for the decrease in difference of noise emissions due to a higher average speed of passenger cars shall be made. Thus, assuming an average of the four noise models with the most congruent results at $80 \mathrm{~km} / \mathrm{h}$, weighting higher the results of NMPB 2008 and CNOSSOS, and accounting for unequal speeds, here the ratio of noise produced by one heavy duty vehicle on average to correspond to the amount of noise of five passenger cars is chosen.

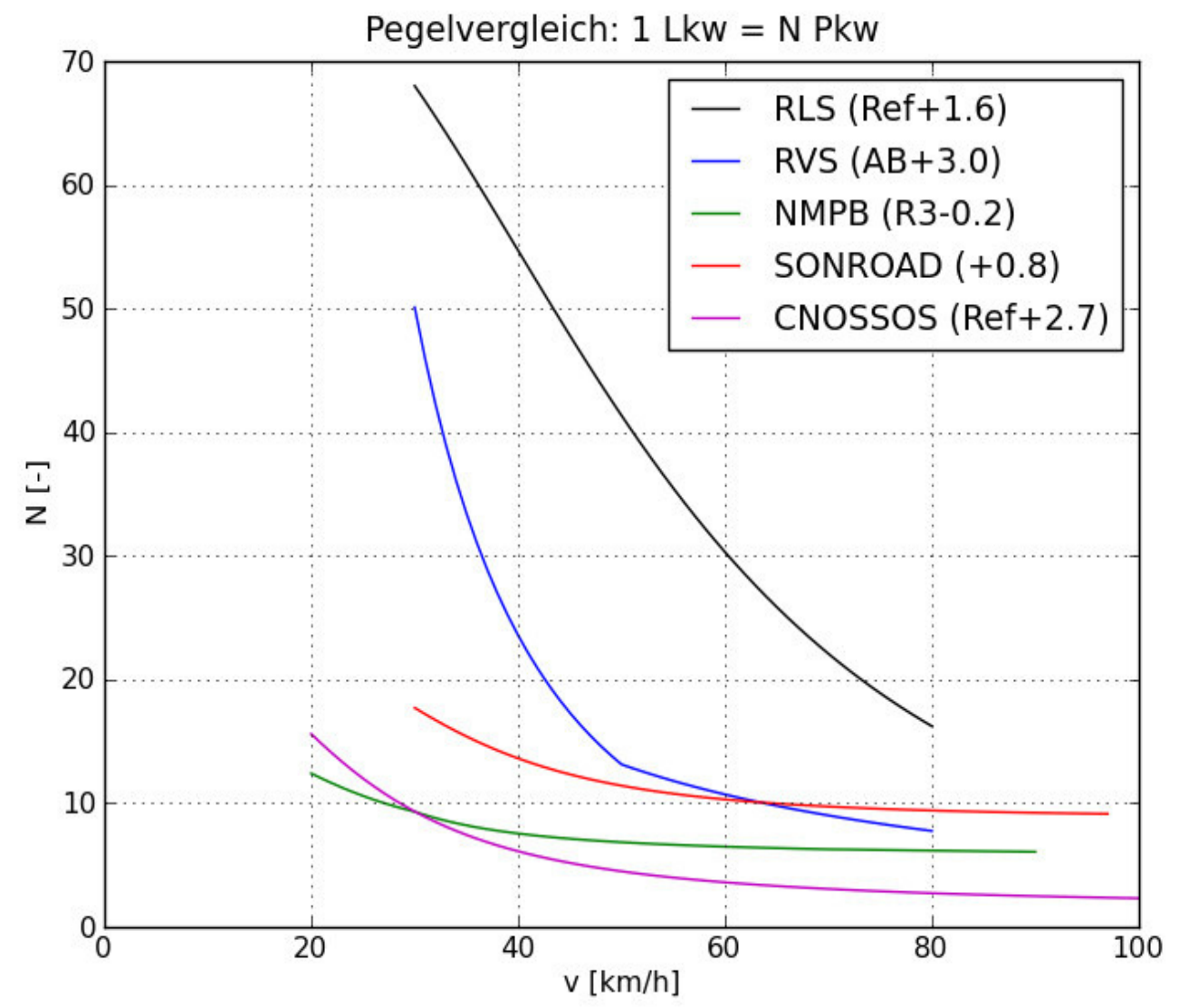

Figure 5.9: Relation of sound pressure levels of passenger cars and heavy duty vehicles as calculated by various emission models used in Europe; source: Bartolomaeus 2010.

While the noise emissions of motorcycles on high traffic roads do not stand out if low speed drives are used, their distinct character of motor sound is perceived as particularly loud in rural or recreational areas with lower use roads (DEGA 2010). Since the 
method at hand considers mainly higher use roads, the impact factor of the vehicle type 'motorcycles' is only raised to a value of 0.8 . The impact factor of buses is decided also to be increased only marginally, namely to a PCU of 3.0, because due to their relatively lower weight and restriction of speed by law they usually do not produce as much noise as heavy duty vehicles. Table 5.15 shows mileages and impact factors of the selected vehicle types.

Table 5.15: Mileage shares and vehicle factors II; mileages adapted from Elsner 2010, vehicle factors assumed from own reasoning.

\begin{tabular}{|r|c|c|c|}
\hline \multicolumn{1}{|c|}{$\begin{array}{c}\text { Vehicle type } \\
\left(\mathbf{v}_{\mathbf{x}}\right)\end{array}$} & $\begin{array}{c}\text { National mileage } \\
\text { in billion tkm. }\end{array}$ & $\begin{array}{c}\text { National } \\
\text { mileage in \% }\end{array}$ & $\begin{array}{c}\text { Vehicle factor/ } \\
\text { PCU } \mathbf{f}\left(\mathbf{v}_{\mathbf{x}}\right)\end{array}$ \\
\hline $\begin{array}{r}\text { Motorcycles }\left(\mathrm{v}_{\mathrm{m}}\right) \\
\text { Buses }\left(\mathrm{v}_{\mathrm{b}}\right)\end{array}$ & 15.4 & 2.23 & $0.8 \mathrm{f}\left(\mathrm{v}_{\mathrm{m}}\right)$ \\
Pick-up trucks $\leq 3.5$ tons $\left(\mathrm{v}_{\mathrm{t} 3.5}\right)$ & 587.5 & 84.90 & $1.0 \mathrm{f}\left(\mathrm{v}_{\mathrm{c}}\right)$ \\
Trucks $>3.5 \leq 12$ tons $\left(\mathrm{v}_{\mathrm{t} 12}\right)$ & 3.4 & 0.49 & $3.0 \mathrm{f}\left(\mathrm{v}_{\mathrm{b}}\right)$ \\
Trucks $>12$ tons $\left(\mathrm{v}_{\mathrm{t}>12}\right)$ & & 5.04 & $2.5 \mathrm{f}\left(\mathrm{v}_{\mathrm{t} 3.5}\right)$ \\
All trucks combined & & 1.67 & $3.5 \mathrm{f}\left(\mathrm{v}_{\mathrm{t} 12}\right)$ \\
Semitrailer tractors $\left(\mathrm{v}_{\mathrm{st}}\right)$ & 60.8 & 2.08 & $5.0 \mathrm{f}\left(\mathrm{v}_{\mathrm{t}>12}\right)$ \\
Other $\left(\mathrm{v}_{\mathrm{o}}\right)$ & 16.9 & 8.79 & \\
Total & 8.0 & 2.44 & $7.0 \mathrm{f}\left(\mathrm{v}_{\mathrm{st}}\right)$ \\
\hline
\end{tabular}

In order to account for the above data, all different vehicle types and their respective impact factors have to be considered by the formula. Hence, the assumed share of a vehicle group (share of $\mathrm{v}_{\mathrm{x}}$ ), which is its average share of mileage of the total national mileage, has to be multiplied by the vehicle factor of that group $\left(f\left(v_{x}\right)\right)$ and the results for all vehicle types have to be added. Hence, the equation to calculate the impact of one heavy duty vehicle has to be modified (see Equation 5.13). This notably changes the impact of one heavy duty vehicle in the assumed situation of a 20 kilometer section and of a daily traffic volume of 50,000 vehicles. The noise driven landscape impact (NDLI) of the heavy duty vehicle increases to $4.9500 \mathrm{~m}^{2}$ from prior $3.0943 \mathrm{~m}^{2}$. The new value is considerably larger because in the prior differentiation between passenger cars and heavy duty vehicles the ratio of the two was more equal than when accounting for the results of the analysis of road noise emission models by Bartolomaeus (2010), which suggests the noise-impact ratio for trucks $>12$ tons and passenger cars to be 5 to 1 . The calculation is indicated below. 
Equation 5.13: Calculation of noise driven landscape impact (NDLI) differentiating between all major vehicle types as of Table 5.15.

$$
\left.\mathrm{NDLI}=\frac{\text { noise zone in meters } \cdot 2 \cdot \text { length of section } \mathrm{i} \text { in meters }}{d t v_{i} \cdot\left[\sum_{x=m}^{o} \text { share of } v_{x} \cdot \mathrm{f}\left(\mathrm{v}_{x}\right)\right] \cdot \text { days of the year }} \cdot \mathrm{v} x\right)
$$

Hence:

Noise Driven Landscape Impact of $1 \mathrm{HDV}=$

$\frac{0,2212 \cdot d t v_{i}^{0.7273} \cdot 2 \cdot 20,000 \text { meters }}{50,000 \cdot[(0.0223 \cdot 1)+(0.849 \cdot 1)+(0.0049 \cdot 2.5)+(0.0504 \cdot 2.5)+(0.0167 \cdot 3.5)+(0.0208 \cdot 5)+(0.0244 \cdot 7)+(0.0116 \cdot 1)] \cdot 365} \cdot 5$

$$
\begin{aligned}
& \Leftrightarrow \quad \text { NDLI of } 1 \mathrm{HDV}=\frac{578.56 \text { meters } \cdot 2 \cdot 20,000 \text { meters }}{50,000 \cdot 1.3544 \cdot 365} \cdot 5 \\
& \Leftrightarrow \quad \text { NDLI of } 1 \mathrm{HDV}=\frac{24,470,800 \mathrm{~m}^{2}}{67.725 \cdot 365} \cdot 5 \\
& \Leftrightarrow \quad \text { NDLI of } 1 \mathrm{HDV}=4.9500 \mathrm{~m}^{2}
\end{aligned}
$$

Comparing the two options, which are either to differentiate only between HDVs and non-HDVs using available, very precise road specific data on their shares of traffic, or to distinguish between all major vehicle types using average national data, option one is more fair with regard to the share of impact of an individual user on a particular road because the most relevant difference in effect is between heavy duty and non-heavy duty vehicles. Moreover, data availability on the shares of heavy duty vehicles on federal highways and freeways is good as the information comes from the same source as does the data on daily traffic volumes, the Federal Highway Research Institute of Germany (Bast 2011a \& 20011b). The data indicates specific shares for sections between all ingress and exit ramps. Option two is more fair with regard to the differentiation between all vehicle groups because for instance a motorcycle has a significantly lower impact than a car and a semitrailer tractor a higher impact than a truck. This differentiation is not possible to be made under option one.

Deducing heavy duty vehicle shares from their national mileage as done in option two may underestimate the impact of heavy duty vehicles because the total mileage of vehicles of all vehicle types accounts for all roads, with smaller roads generally having lower heavy duty vehicle shares than freeways and federal highways, which are the road types focused upon here as they are most used for freight transport. This can be seen when adding the mileage percentages of trucks and semitrailer tractors as of Table 5.15 . Their total percentage is $11.23 \%$, which is a slightly lower value than the expected average share on freeways (14.56\%) and federal highways (10.11\%) (see Ta- 
ble 5.7). However, the loss of precision of an individual road or road section is viewed less distortive as is the gain to more precisely determine the impact per vehicle type regarded beneficial. In addition, while a higher accuracy from assuming real heavy duty vehicle shares for a particular road as in option one may seem relevant when accounting for spatial ecological impact in route selection, the distortive impact from assuming a potentially imprecise share of heavy duty vehicles as in option two is negligible compared to the two most weighty factors of traffic volume and vehicle type and potentially the presence of mitigation barriers in the future. For a company, a more precise calculation of impact per vehicle type is also beneficial in order to yield higher accuracy for the measurement of the specific type of heavy duty vehicle used in order fairly to relate the impact to single products. The final NDLI-formula (noise driven landscape impact) allows determining the landscape impact per vehicle $\left(\mathrm{f}\left(\mathrm{v}_{x}\right)\right)$ for an entire route. Optionally, in order to obtain a serviceable yearly result for reoccurring trips, the result can be multiplied by the number of trips of a given vehicle on that route during one year. A route consists of various sections that are determined as homogeneous sections by the characteristic of containing identical traffic volumes. When traffic volume changes a new section begins. Other factors that potentially speak in favor to distinguish between sections, such as surrounding urban area or the presence of noise mitigation barriers, are not considered because aberrations of the impacts are reasoned not to be significant enough necessarily to be accounted for individually and because data availability is not good. The final formula recommended to be used for the quantification of degrading, predominantly noise driven impact on adjacent area is shown in Equations 5.14. The measuring unit of the result is square meters.

Equations 5.14: Calculation of noise driven landscape impact (NDLI) per vehicle per route.

a)

$$
\mathrm{NDLI}=\sum_{i=1}^{n}\left(\frac{0,2212 \cdot d t v_{i}^{0.7273} \cdot 2 \cdot d_{i}}{d t v_{i} \cdot\left[\sum_{x=m}^{o} \text { share of } v_{x} \cdot f\left(v_{x}\right)\right] \cdot 365}\right) \cdot f\left(v_{x}\right)
$$

b)

$$
\mathrm{NDLI}=\sum_{i=1}^{n}\left(\frac{0,2212 \cdot d t v_{i}^{0.7273} \cdot 2 \cdot d_{i}}{d t v_{i} \cdot(1.3544) \cdot 365}\right) \cdot f\left(v_{x}\right)
$$

$\mathrm{i}=$ homogenous section $\mathrm{i}$ with $\mathrm{i}=1,2, \ldots, \mathrm{n}$

$\mathrm{n}=$ total number of road sections considered

$\mathrm{dtv}_{\mathrm{i}} \quad=$ average daily traffic volume of section $\mathrm{i}$

$\mathrm{d}_{\mathrm{i}} \quad=$ distance travelled in section i in meters

$\mathrm{v}_{\mathrm{x}} \quad=$ vehicle type

$\mathrm{v}_{\mathrm{x}=\mathrm{m}, \ldots, \mathrm{o}}=$ all existing vehicle types

$\mathrm{f}\left(\mathrm{v}_{\mathrm{x}}\right)=$ vehicle factor of vehicle type $\mathrm{v}_{\mathrm{x}}$ 


\subsubsection{Possibilities of impact mitigation}

An array of options to mitigate noise from road traffic exists. Road users intuitively perceive most measures to lie out of their reach. While some clearly are at the hands of infrastructure planners and operators, such as implanting quieter road surfaces, installing artificial or natural sound barriers like soil berms or placing shielding and absorptive vegetation, and other technical options are at the hands of vehicle manufacturers, like developing quieter engines, tire designs, or vehicle aerodynamics, road users can make a significant contribution in what could be called 'traffic behavior' (Forman et al. 2002, Parris \& Schneider 2009, Blickley \& Patricelli 2010). This refers to: firstly, the distribution of traffic in a road network, when a bundling of traffic on fewer roads in most cases is ecologically more efficient than spreading traffic throughout a rather dense network, secondly, vehicle speed, thirdly, the willingness to accept temporary seasonal or daytime related closures up to the removal or permanent public closure of certain stretches, and fourthly, considering the question, whether in spite of higher costs or lower revenues transport intensity can be decreased. While incentives or restrictions implemented by national and regional infrastructure planners would give guidance and allow for more fair national competition, companies can adjust their behavior on their own initiative. They will then have to communicate their decisions to stakeholders in order to explain prize premiums or constraints to revenues.

With regard to all other impacts that together with noise create a cumulative spatial impact of road transport, other individual options of mitigation exist. Problematically, most technical measures mitigate one, but reinforce another impact, for instance as fencing reduces mortality, but enhances the barrier effect. Again, some measures are more closely connected to road operators and vehicle manufacturers and others to users. Contributing to a bundling of traffic and reducing haulage distance per product are the most effective options to abate all impacts. In the next subchapter on fragmentation, two further methods that measure the barrier effect are introduced.

\subsection{Quantification of Fragmenting Impact from Road Transport}

The second underrepresented ecological impact in corporate and also in national environmental management is the fragmentation of the landscape, a landscape that despite being semi-natural, still contains different habitats for animal and plant species. Like land use, fragmentation also is a spatial impact. In the description of the impact in section 4.3 it was said that the ecological impact of fragmentation is difficult to measure. On the one hand this is because the impact depends on the overall degree of fragmentation of an area, on the intensity of human use of an area, and on the habitats and 
species present in certain locations. On the other hand, study designs are complex and too costly to be carried out comprehensively over a large area. Unlike in the rather straightforward case of degraded area, the situation of imperfect knowledge on the differing degrees of impact for differing species and the difficulty to determine a clear cause-and-effect relation of a road user's impact compared to that of the condition of the larger landscape on ecological processes make a precise correlation between an individual vehicle and the fragmenting impact rather challenging. Thus, both approaches of quantification introduced here are no more than rough estimates of describing the impact of particular roads and individual users. They are the first proposals that on the one hand can serve corporate decision-makers who want to report and consider a more complete set of environmental impacts associated with the production and delivery of their products and that on the other hand can activate and inspire researchers and privates who are working in field of environmental management to develop the methods further in order to eventually agree on a methodology that can be standardized, offered, and demanded to be applied and reported by the majority of businesses and institutions. Of the two approaches presented in the following, the first one regards fragmentation from a rather top-down, conservation-strategic, and supraregional connectivity-centered perspective and the second one is more suitable to represent the impact at an unweighted, discretionary, and total landscape scale.

\subsubsection{Determination of the Fragmenting Impact of a Road User}

It is not possible to merge the two approaches indicated above to obtain one final result. This predominantly is not because of dissimilar measuring units, but due to the different perspectives of analysis that both approaches hold. The first approach gauges fragmentation by the impact of transport infrastructure on wildlife corridors. Compared to the second approach, which measures the fragmenting impact for an entire landscape independent of the ecological value of a location as a habitat or corridor and hence considers every kilometer travelled, the first approach in focusing on the seemingly most important trait of habitat connectivity, that being most frequently used corridors, prioritizes certain locations in a landscape over others. While this has the advantage most effectively to help species and supposedly most efficiently to dedicate resources to mitigating the impact of fragmentation on the diversity, abundance, and well-being of species, the approach bears the risk to communicate and to provoke a narrow-minded and anthropocentrically, utility driven thinking of the role and the demands of ecosystems and non-human species. This is a critique of corridor driven approaches only insofar, as they fall short to emphasize the equal importance of also maintaining small and particularly large ecologically valuable areas because not all species can disperse via the crossing structures provided and because, ultimately, 
movement corridors that allow for connectivity only are relevant if there are things to be connected. As national parks alone fail to function as databases of regional diversity, larger rather permeable areas that are relatively undissected by intensive land use, settlement area, or traffic infrastructure, are also highly valuable areas because they were found to facilitate ecosystem processes, ecosystem integrity, and ecosystem stability, including the preservation of native biodiversity and the rendering of ecosystem services vital for human societies (Selva et al. 2011). This is why the easier manageable approach of measuring the impact of the barrier effect from road traffic by the means of habitat corridors is complemented by an approach that does not distinguish between areas perceived as more or less important for connectivity and that thus equally considers the demands of movement of all species in all locations of the still largely semi-natural landscape of Germany. The two corridor analyses to be referred to at least insofar argue in favor of large undisturbed areas as they determine vast lists of conflicts to be attended to and do not stop at a threshold of for instance the 100 most urgent conflicts. Discussion on the dissimilar approaches of impact assessment followed in this work similarly occurs in analyses that are intended to determine the most effective and most appropriate strategies to mitigate the barrier effect at the landscape level and to restore a certain degree of connectivity. An examination of the two most common, complementing, and yet rivaling strategies of defragmentation is undertaken in Friedrich and Geldermann 2013b (see Article II). In the following two sections, two methods that allow approximating the fragmenting impact of a road user driving a particular route are introduced. At first, a corridor based quantification, and subsequently, in section 5.2.1.2, a landscape discretionary quantification are pursued.

\subsubsection{Corridor- and prioritization-based quantification}

The first approach that considers habitat fragmentation from a coarser and more functional perspective is based on a work from Hänel and Reck (2011). In their analysis the authors consider the major known wildlife corridors of species of the four major habitat types of terrestrial Germany as well as the German grid of transport infrastructure, and here particularly larger roads. Also considered are some local habitats that are dissected by roads. However, as these habitats are not part of supra-regional corridors, which are the focus of the work, the conflicts of fragmentation of these habitats are not assessed in detail and are ranked to be of lower importance. Comparing both infrastructures, corridors and roads, Hänel and Reck were able to find locations of conflict at which the two infrastructures collided or intersected. A road is considered a barrier when the average daily traffic volume was 1,000 vehicles or higher. This minimum threshold is increased to 5,000 vehicles for the habitat corridors of large mammals. Additionally considered elements are existing artificial structures targeted to allow road 
crossings, the network of the most used and at least electrified and double-tracked railway lines, and the impact of intensively used or built-up canals, which per definition are assumed to be all federal waterways of category IV or higher. As the intention of Hänel's and Reck's work was not only to identify locations of conflict between ecological connectivity and road traffic, but also to suggest abatement of the conflicts, they used the various criteria that allow determining a conflict also to determine a conflict's severity in order to rank conflicts and to disclose lists of priority for mitigation.

The criteria to determine a conflict and its severity vary per type of habitat. Yet, the following three main criteria to differentiate between differing degrees of conflict were used: first, the ecological importance of a habitat location or corridor, which inter alia is determined by the size and the proportion of resulting fragments, with aliquot dissection being worse than dissection into two unequally sized fragments, by the importance of the species, for instance determined by the characteristics of a species as being rare or being a vector or target species, and by the importance of a location as a core area or as a larger landscape corridor; second, the severity of the barrier function of a road, railroad, or waterway, which is determined mainly by the intensity of use, but also by width and fencing construction of the infrastructure; and third, existing opportunities for wildlife to cross an infrastructure, which are assessed mainly by the width of underground or aboveground structures (Hänel \& Reck 2011).

With regard to the courses of the different habitat corridors, Hänel and Reck build on a study that established a national cartographic overview of habitat corridors of wet habitat, dry habitat, coastal habitat, and of habitat of forest and semi-open landscape living species (Reck et al. 2005). The four types of habitat corridors considered in the analysis of Hänel and Reck hence are: wet habitats, dry habitats, at least seminatural forest habitats, and habitats of forest living large mammals. Information on the grid of roads with an average traffic volume of more than 1,000 vehicles per day came from a research project of the Federal Agency for Nature Conservation, which in turn mainly drew from a work of Esswein and Schwarz von Raumer (2005). Ground cover was assumed as of Corine Land Cover 2000 for Germany (UBA \& DLR-DFD 2004) and as of Corine Land Cover 2000 Europe for the neighboring countries (EEA 2002). CORINE stands for 'Coordination of Information on the Environment'. The assembly of this cartographic database was originated by the European Commission in order to obtain a consistent classification of the major types of land use. Further data on water bodies, settlements, and national borders came from the Federal Agency for Cartography and Geodesy (BKG 2008). The same institution provided cartographic data on existing road crossing structures (BKG 2006). This strain of data was complemented by information from the Federal Highway Research Institute (Bast 2014). Data was obtained or transferred into a format so it could be used in geographic information system modelling. 
Hänel and Reck present their findings in catalogues, ordered by priority, and in maps of all of Germany. Also in the maps, the locations of conflict are indicated as of their priority, 1 representing the highest and 5 the lowest priority. Locations of conflict that are not numbered indicate that the locations were not yet classified, but overall were assumed to be of subordinate importance to priority 5 . The maps further show crossing structures that either are in place or are planned by the symbol of a green star. The maps have green-marked points to indicate locations of tunnels and viaducts. Furthermore, the corridors of concern, continuous natural areas of ecological value, and basic topographic information on forests, rivers, and waterbodys, are indicated. In addition, areas and elements of human use, such as settlement area, roads with more than 1,000 vehicles per day, and railway lines, as well as names of larger cities and federal and national borders are depicted. Due to the display of a large quantity of information and the objective to maintain good visibility, no document exists where the locations of conflict of all four habitat types are aggregated in one map. To obtain an impression about the character of the maps see Figure 5.10. It is a cut-out of the map for large mammals.

Table 5.16 indicates the number of conflicts indexed by priorities of the four different habitat types of the corridors. The numbers do not match the quantity of conflicts indicated in Hänel's and Reck's main publication because the data in Table 5.16 refers to all identified conflicts, whereas for the sake of the publication, the locations were analyzed further and partially were consolidated into fewer instances of conflict (2011, pp. $204 f$ and 239). Also applying the rules stated on the following pages on how to read the maps in order to achieve a quantification of a value of fragmenting impact, less conflict points than indicated in Table 5.16 are effective. Furthermore, it shall be noted that despite the conflicts of the habitat types of large mammals and forest habitat in some instances refer to an identical location, it is viewed appropriate to view them as distinct events because they focus on different species. While the impact of waterways and railroads is taken into account to determine the severity of a conflict, locations of conflict are assigned only to roads.

Table 5.16: Number of conflicts determined by Hänel \& Reck by habitat type and priority.

\begin{tabular}{|lrrrrrc|}
\hline $\begin{array}{c}\text { Habitat type of } \\
\text { the corridor }\end{array}$ & $\mathbf{1}$ & $\mathbf{2}$ & $\mathbf{3}$ & $\mathbf{4}$ & $\mathbf{5}$ & $\begin{array}{c}\text { Sum of priori- } \\
\text { tized conflicts }\end{array}$ \\
\hline Large mammals & 25 & 106 & 352 & 457 & 1,429 & 2,369 \\
Forest habitat & 22 & 38 & 99 & 119 & 430 & 708 \\
Dry habitat & 24 & 24 & 37 & 149 & 53 & 287 \\
Wet habitat & 16 & 59 & 70 & 171 & 396 & 712 \\
\hline
\end{tabular}




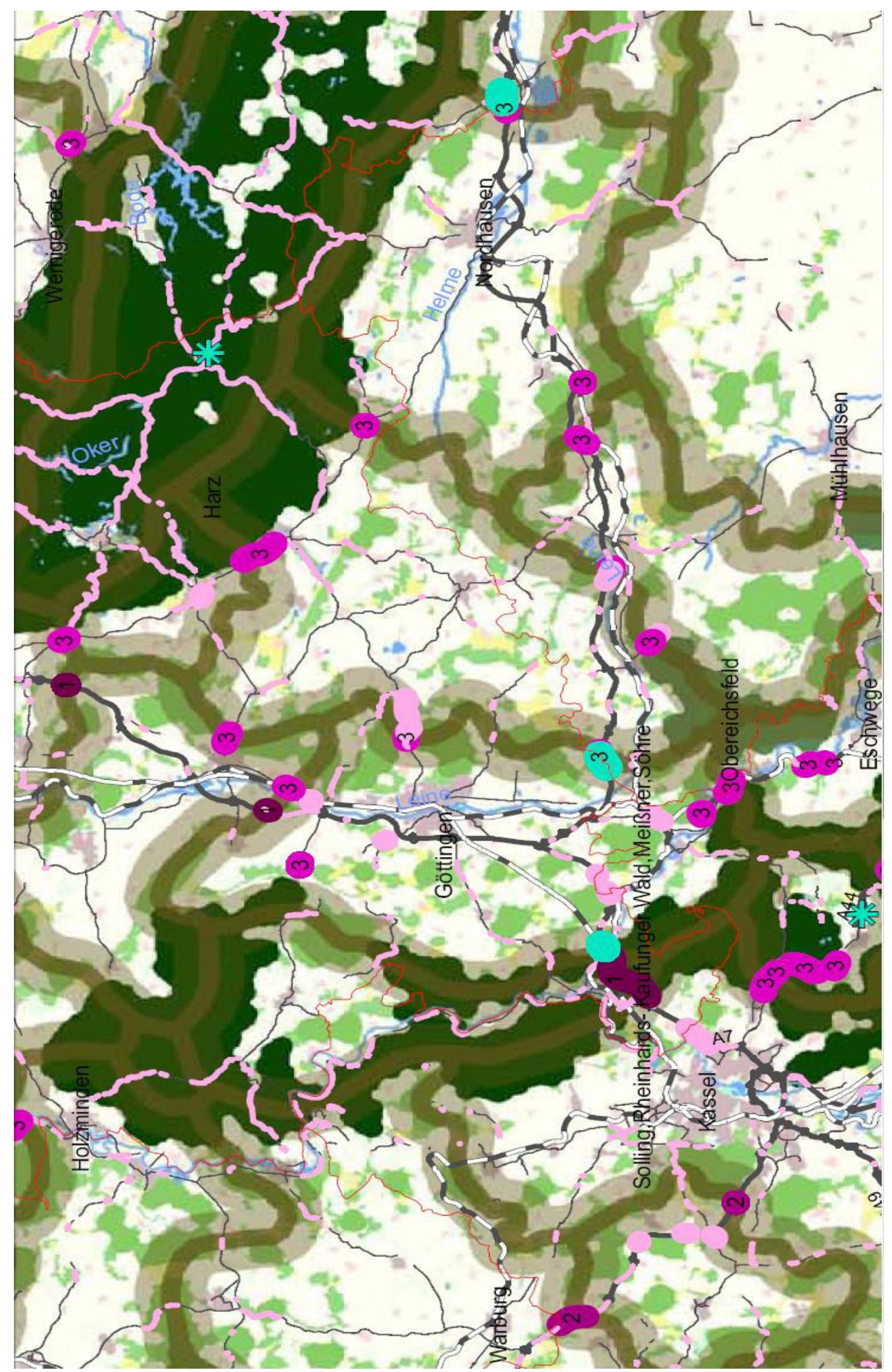

Figure 5.10: Cut-out of the map for large mammals for the region around Göttingen in Lower Saxony, 156 x 74 kilometers; source: Hänel and Reck 2011. 
Prior to Hänel and Reck, Herrmann et al. (2007) had analyzed wildlife corridors and traffic infrastructure at the German national level. In dependence on the societally attended to Federal Transport Infrastructure Plan, the study is entitled Federal Wildlife Infrastructure Plan (FWIP). The study emphasizes that in a landscape different levels of connectivity exist, which concern both large and short distance demands of animals. The scope of the FWIP was the national level, which means that European corridors on the one hand and regional and local corridors and habitats on the other hand were not accounted for satisfactorily. The types of traffic infrastructure that were considered are high-traffic railways and large federal roads, i.e. federal highways and freeways with a traffic volume higher than 10,000 vehicles per day. The corridors were determined assuming two types of sources of input, existing corridor studies from Reck et al. (2005), Strein et al. (2005), and Reuther \& Krekemeyer (2004) on the one hand, and information gathered by in-house specialists on the dispersal of five keystone species, which were wild cat (Felis silvestris), lynx (Lynx), wolf (Canis lupus), red deer (Cervus elaphus), and otter (Lutra lutra), on the other hand. Together the information yielded maps of corridors for species of forest, half-open, dry, and wet habitats.

The FWIP determined 900 points of conflict between the habitat corridors and the traffic infrastructure considered. The conflicts were weighted by 21 criteria, of which the importance and width of a corridor, the intensity of traffic of a transport infrastructure, and recommendations of experts were the three most important criteria. In the end, 125 locations were identified to be of highest priority for mitigation. Hänel and Reck exemplarily compared their findings to those of the Federal Wildlife Infrastructure Plan. For the habitat type of forest living large mammals 9 of the 14 locations identified as being of highest priority were identical to the ones identified to be among the 125 locations of priority of the FWIP. Summing the locations of priority groups 1 and 2, still more than $50 \%$, namely 34 of 65 conflicts, are identically classified under the 125 priority locations of the FWIP. Furthermore, apart from very few exceptions, for instance for locations at railroads that were considered, but not designated as locations of conflict by Hänel and Reck, all other of the 125 locations of priority identified under the FWIP are listed under the priority groups 3,4 , and 5 of the study of Hänel and Reck. This relatively large congruence not only of locations detected, but also of the similar assignation of priorities would be close to $100 \%$ had the authors of the FWIP not targeted a distribution of the conflicts proportionate to federal states (Hänel \& Reck 2011).

The Federal Wildlife Infrastructure Plan was the first study of its kind with a national scope. As its input data with regard to the corridors was still incomplete and constricted to a national focus, as the methodology and the output data are less detailed as in the work of Hänel and Reck, and as their later analysis recognizes the findings of the 
FWIP, the work of Hänel and Reck is viewed superior and it in the following is used to determine the fragmenting impact of an individual road user.

As for the indicators of land use, vehicle type is accounted for by the means of passenger car units. The factors are those assumed for the measurement of the share of sealed area in section 5.1.1 (see detailed reasoning later in this section). The share of an individual user of the total fragmenting impact is not calculated. This is because the measuring unit does not represent a real ecological impact, only which would make a fair allocation of the impact more desirable and feasible. In order to calculate the impact of a given vehicle on a given route, the locations of conflict along that route have to be extracted from the four maps. No tables exist in which the locations of conflict are organized in a way that would make their identification easier for the purpose at hand. This is because the locations of conflict were not determined to describe certain routes, but the goal was to identify locations of conflict and to prioritize them to some extent in order to obtain an overall impression of the relevance of fragmentation and in order to begin mitigation and to dedicate financial resources most efficiently. In addition, roads were not always labeled because the geo-referenced data on traffic volumes of the road grid was not obtained as one uniform dataset, but came in in different levels of detail from the federal states, meaning that. A future update of the results of the study would use a current version of the "NeMoBFStr", a network-model for federal roads that was not completed at the time of the study (BMVBS 2012b). The model contains datasets that have uniform entries and that have much higher degrees of annotation that would make it easier to display results in various types of catalogues.

Thus, for the time being, the more tedious approach of extracting the information for a particular road from the maps has to be applied. Here the following difficulty arises: oftentimes locations of conflict either lie close together or are meant to describe one continuous conflict and are hence much longer than an individual conflict point. In both cases it is sometimes difficult to know how the line of conflicts should be understood, whether it should be regarded as just one conflict or whether two or more conflicts apply. One such extending location of conflict is the entry of priority group 1 of Freeway 7 and the corridor of large forest living mammals of the Forest of Kaufungen (German: Kaufunger Wald) north-east of Kassel, Germany (see Figures 5.10 and 5.11). Depending on which parts of the forest are included in the corridor, the line of conflict is between 6.5 and 11.5 kilometers long. This suggests the situation of conflict be regarded to be more severe than a less extensive conflict. To this end, a more detailed local analysis of the use of an area, the demands of the existing species, the topography, existing possibilities for crossings, and the feasibility of potential means of mitigation would be necessary. While the findings of Hänel and Reck (2011) can initiate the overall process of mitigation, they hold no information of this level of detail. This for instance 
concerns the placement and quantity of structures needed in situations of extending locations of conflict, which would be an indication, how the severity of an extending location of conflict should be understood. With regard to the construction of the various existing types of crossing structures, a number of aspects important for the use and functionality of a particular structure have to be considered. See Reck et al. (2011) and Friedrich and Geldermann (2013b) for a set of guidelines on the planning and construction of large bridge-type structures.

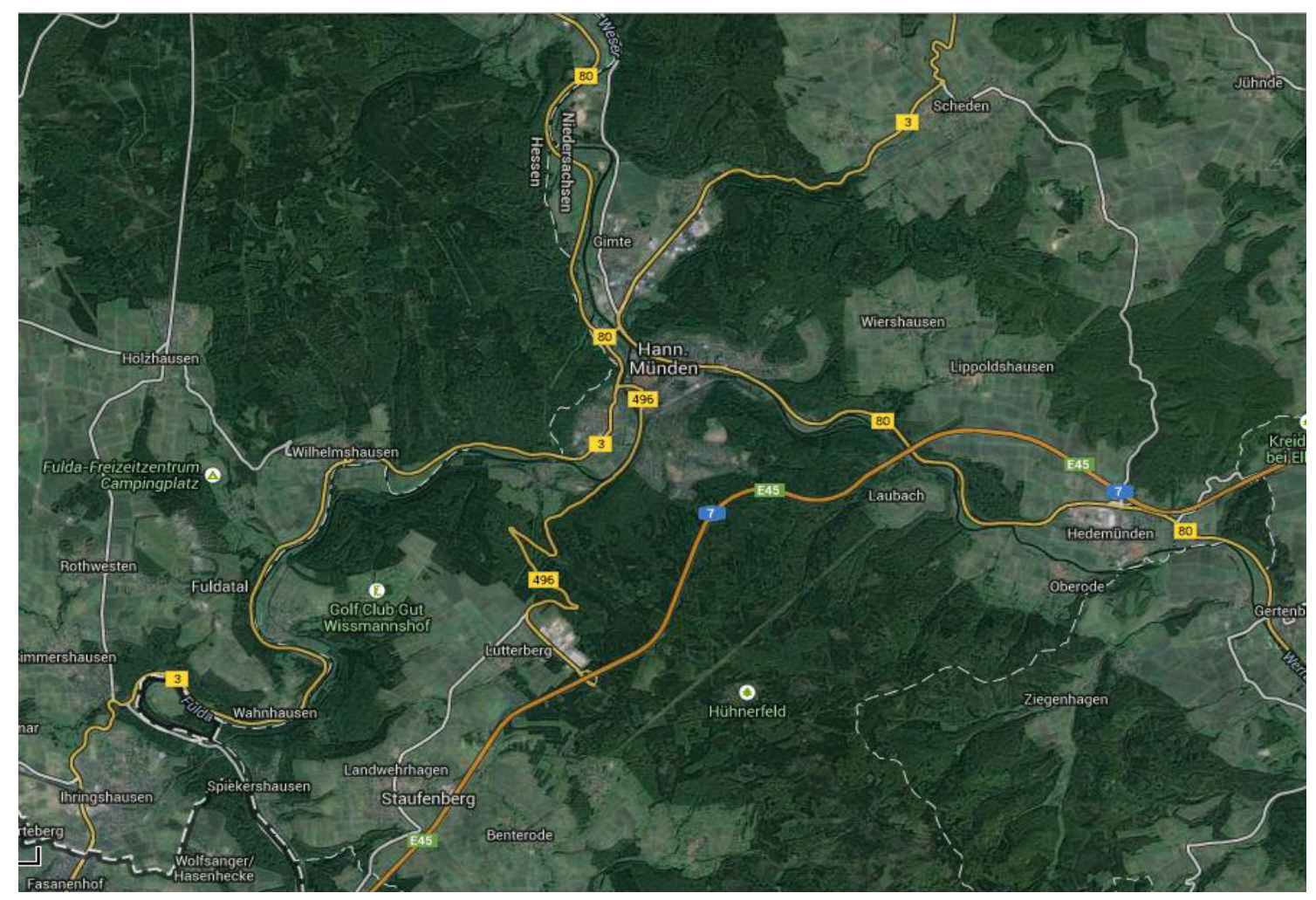

Figure 5.11: Image of an extending zone of conflict between Freeway 7 and the habitat corridor of forest living species north-east of Kassel, Germany; source: Google Maps.

The easiest way to proceed in a case where a line of conflicts renders the quantity of conflict points unclear, would be to define a maximum length over which an area of conflict can extend still to be regarded as a single incident and from which length onwards a line of conflicts has to be considered to represent two or more conflicts respectively. However, this is not easily possible because the demands for connectivity vary between species and depend on individual topography. Estimations on an appropriate distance between opportunities of crossings vary between 1.6, 5, and 10 kilometers. Bissonette and Adair (2007) found that for North America, gaps between crossing structures that would be no larger than $1.6 \mathrm{~km}$ would serve about $71 \%$ of the $72 \mathrm{spe}$ cies considered in their study. While this placing distance might be appropriate for 
hotspots and in order to establish conditions close to permeability, even such a high density is likely not to meet the demands of all species sufficiently and it is unfeasible to be applied to an entire road grid. Hence, so far the strategy is to gear the configuration and location of individual structures to the species with the highest demands and of the lowest mobility. In the next paragraph, the code of practice for the counting procedure of conflicts from the maps is indicated.

In order to identify and to count all conflicts that lie on a given route, the following four rules shall be effective: firstly, locations of conflict are to be considered as two dissimilar cases when they are not connected. Secondly, extending locations of conflict shall not be considered as one event of conflict, if it is clearly visible that they are valid for the connectivity of different habitats or corridors. They shall then be counted as one conflict per connection of dissimilar habitats or corridors (see Figure 5.12). Thirdly, in order to account for the severity of the conflicts, the approach of quantification suggested in this work designates to multiply the sum of conflicts of each priority group by the following values: the sum of conflicts of the highest group of priority (Group 1) are to be multiplied by a factor of five, the second most severe conflicts of Group 2 are multiplied by a factor of four, conflicts of Group 3 by three, conflicts of Group 4 by two, and conflicts of Group 5 by one. Conflicts that were not classified but are indicated nonetheless shall form Group 6 and be multiplied by a factor of 0.25 . The presence of viaducts, tunnels or green-bridges, also referred to as 'wildlife crossings', was considered in the analysis of Hänel and Reck (2011), hence, their alleviating impact is assumed to be recognized sufficiently.

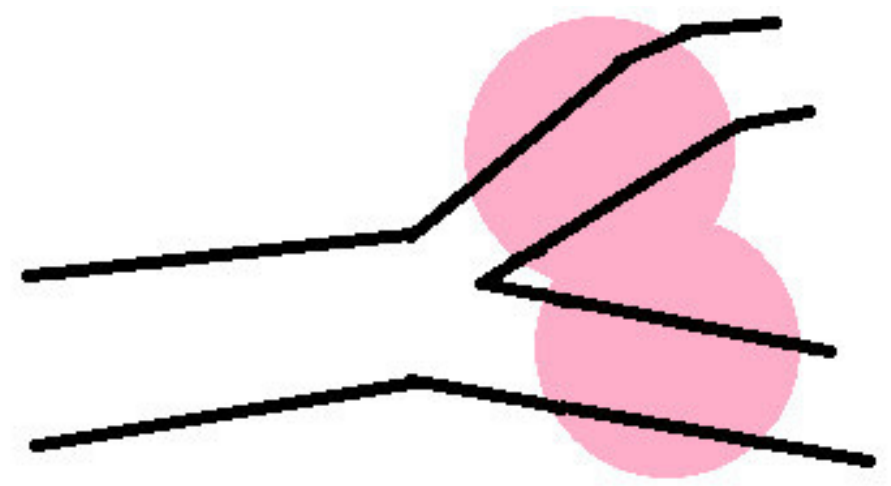

Figure 5.12: Two locations of conflict forming one line conflict, but to be counted individually as they concern distinct corridors.

For a given route, this procedure has to be applied to all four maps that indicate conflicts between road traffic and a habitat corridor (see Hänel et al. 2010). The conflicts of category five are not numbered due to their quantity and due to the visibility of the 
graphics. Yet, they can be identified as it is intuitive that they are the ones indicated in lightest green and lightest turquois. All other markings in the colors of the conflict points are much smaller. They represent all conflicts that were not sufficiently assessed to be classified to belong to one of the five groups of priority. However, they are estimated generally to be less severe than the least severe points of conflict of Group 5, which is why in the analysis at hand they are designated to form Group 6.

When quantifying the fragmenting impact of a road user, a differentiation by vehicle type is suitable because the increased taking up of space and generation of noise of larger and heavier vehicles produce a larger barrier effect than do small vehicles. Noise cannot only reduce habitat quality or lead to a loss of habitat, but in deterring species and individuals also can contribute to the barrier function of a road. Yet, regarding the assumption of vehicle factors, the quantity and size of vehicles and the configuration of a road for most species represent the more decisive parameters that render a road unpassable. Thus, in spite of noise contributing to the barrier effect, here the vehicle factors assumed for the allocation of sealed area, are decided to be used. The factors are identical to the ones determined to represent the impact of dissimilar vehicle types on land use in section 5.1.1. They are reprinted in Table 5.17.

Table 5.17: Mileage shares and vehicle factors III; adapted from Elsner 2010, Bast 2005, Delft et al. 2011, ProgTrans AG and IWW 2007.

\begin{tabular}{|r|c|c|c|}
\hline \multicolumn{1}{|c|}{$\begin{array}{r}\text { Vehicle type } \\
\left(\mathbf{v}_{\mathbf{x}}\right)\end{array}$} & $\begin{array}{c}\text { National mileage } \\
\text { in billion tkm }\end{array}$ & $\begin{array}{c}\text { National } \\
\text { mileage in \% }\end{array}$ & $\begin{array}{c}\text { Vehicle factor/ } \\
\text { PCU } f\left(\mathbf{v}_{\mathbf{x}}\right)\end{array}$ \\
\hline Motorcycles $\left(\mathrm{v}_{\mathrm{m}}\right)$ & 15.4 & 2.23 & $0.5 \mathrm{f}\left(\mathrm{v}_{\mathrm{m}}\right)$ \\
Cars \& Combination vehicles $\left(\mathrm{v}_{\mathrm{c}}\right)$ & 587.5 & 84.90 & $1.0 \mathrm{f}\left(\mathrm{v}_{\mathrm{c}}\right)$ \\
Buses $\left(\mathrm{v}_{\mathrm{b}}\right)$ & 3.4 & 0.49 & $2.5 \mathrm{f}\left(\mathrm{v}_{\mathrm{b}}\right)$ \\
Pick-up trucks $\leq 3.5$ tons $\left(\mathrm{v}_{\mathrm{t} 3.5}\right)$ & & 5.04 & $1.2 \mathrm{f}\left(\mathrm{v}_{\mathrm{t} 3.5}\right)$ \\
Trucks $>3.5 \leq 12$ tons $\left(\mathrm{v}_{\mathrm{t} 12}\right)$ & & 1.67 & $2.0 \mathrm{f}\left(\mathrm{v}_{\mathrm{t} 12}\right)$ \\
Trucks $>12$ tons $\left(\mathrm{v}_{\mathrm{t}>12}\right)$ & & 2.08 & $3.0 \mathrm{f}\left(\mathrm{v}_{\mathrm{t}>12)}\right)$ \\
All trucks combined & 60.8 & 8.79 & \\
Semitrailer tractors $\left(\mathrm{v}_{\mathrm{st}}\right)$ & 16.9 & 2.44 & $4.0 \mathrm{f}\left(\mathrm{v}_{\mathrm{st}}\right)$ \\
Other $\left(\mathrm{v}_{\mathrm{o}}\right)$ & 8.0 & 1.16 & $1.0 \mathrm{f}\left(\mathrm{v}_{\mathrm{o}}\right)$ \\
\hline Total & 692.0 & 100.01 & \\
\hline
\end{tabular}

The vehicle factor completes the assembly of variables needed to determine a fragmenting value of a given vehicle driving a given route when using a corridor- and habitat-orientated approach ( $\left.F V_{\text {cha }}\right)$. The final formula is indicated in Equation 5.15. Users, who repeatedly drive a certain route and who are interested in yearly totals and not primarily in the comparison of two routes, can multiply the obtained result by the quanti- 
ty of trips per year. It again shall be pointed out that this approach of quantifying the fragmenting impact of an individual road user predominantly had two goals: one was to illustrate the impact in a plausible way in order to sensitize road users and consumers who use products with embodied road travel for the existence of the impact. The second was to provide a rough method of assessment and quantification to those who wish to consider, communicate, and mitigate the impact. The indicator allows for that. Yet, as the impact of fragmentation is complex, the indicator is incapable precisely and comprehensively to describe the ecological impact of driving on a road and producing various forms and implications of the barrier effect to many species at the individual and population level, today and in the future, in the end also concerning entire ecosystems and landscape stability. This is why the measuring unit of the result of the method is referred to as fragmenting value, which appears to be less definite than fragmenting impact. The result has no spatial or species diversity-specific implication, but is plainly numeric. The calculation of a fragmentation value applying a corridor- and habitatorientated approach is recommended to be complemented by the indicator presented in the next section. This is not so much for the results, which are not comparable, but for a dissimilar approach that is applied to define the impact. This shows in a different method of calculation, which in turn has significant implications on the interpretation and communication of the results and the mitigation of the impact.

Equation 5.15: Quantification of the fragmenting impact of an individual road user when applying a corridor-orientated assessment of impact.

$\mathrm{FV}_{\text {cha }}=\sum_{w, d, f, l m}^{h}\left(x_{c g_{1} h} \cdot 5+x_{c g_{2} h} \cdot 4+x_{c g_{3} h} \cdot 3+x_{c g_{4} h} \cdot 2+x_{c g_{5} h}+x_{c g_{6} h} \cdot 0.25\right) \cdot f\left(v_{x}\right)$

$\mathrm{FV}_{\text {cha }}=$ fragmenting value using a corridor- and habitat-orientated approach

$\mathrm{h} \quad=$ habitat type: wet $(\mathrm{w})$, dry $(\mathrm{d})$, forest $(\mathrm{f})$, and forest living large mammals $(\mathrm{Im})$

$\mathrm{x}_{\mathrm{cgih}}=$ number of occurrences of conflicts of habitat type $\mathrm{h}$ in conflict group $\mathrm{i}$

$\mathrm{cg}_{\mathrm{i}} \quad=$ conflict group $\mathrm{i}$ indicating the severity of conflict from high to low, $\mathrm{i}=1, \ldots 6$.

$\mathrm{f}\left(\mathrm{v}_{\mathrm{x}}\right) \quad=$ vehicle factor of vehicle type $\mathrm{v}_{\mathrm{x}}$ as of Table 5.17

\subsubsection{Landscape discretionary quantification}

A second approach of representing and measuring the fragmenting effect of a road bound transport is considered indispensably to complement a corridor- and prioritybased approach in order to account for the fragmenting impacts that are effective in any location of a semi-natural landscape, including those sites where crossing structures are installed, but are not used by the entirety of species of a certain habitat. The formula of measurement to be suggested thus has to consider all sections of a route 
over a road's total length equivalently and it has to account for the severity of the impact in accordance to the respective road that is being used. The latter is important because the fragmenting impact of a high-traffic road, i.e. with a daily traffic volume of 30,000 vehicles or higher, has a more severe fragmenting impact than a lower use road that for instance is used by 3,000 vehicles per day. In the corridor-based quantification, no further differentiation of the impact by road type was necessary because this criterion was considered in the determination of priority levels. Assuming an approach that regards a landscape discretionarily, that is considering all locations equally and not favoring one over another, the severity of the fragmenting impact of a road can be accounted for by the traffic volume of the road. It shall be clarified that the impact measured in the following solely refers to the barrier effect of a road and not to the overall ecological impact of a road at a certain traffic volume. Nonetheless, statistics and thresholds on mortality determined in other studies are important, because severe rates of mortality as occurring in some amphibian, reptile, or small mammalian species, can turn a road into a barrier that is severe enough not only to yield high death rates, but also to produce all other impacts associated with the barrier effect that usually occur due to deterrence or physical hindrance, particularly with regard to the potential impacts on genetic variability.

It is difficult and there is hardly a right or wrong, when estimating levels of severity of fragmenting impact from levels of traffic volume because traffic thresholds that represent a barrier vary significantly between species. Here, the to date still most extensive literature review of Charry and Jones (2009) on the impact of different levels of traffic volume on most classes of animals, i.e. amphibians, reptiles, carnivores, ungulates, and birds, a later assessment of Forman and Sperling (2011) that to some extent evaluates the conclusions drawn by Charry and Jones (2009), and a number of individual studies, build the basis for the estimation of levels of severity of impact assumed in this work.

Despite the variation of impact between species, here the severity of fragmenting impact is determined subject to traffic volume. The major guiding hypothesis for the weighting of impact is the assumption that higher volumes are always more fragmenting than lower volumes. While this is assumed to be valid for the overall impact of fragmentation, it does not necessarily apply to the well-being of individual or the shortto medium-term fitness of populations because both can be higher when higher traffic levels deter animals from roads and prevent their death in collisions. However, in total the impact of a barrier function is viewed more harmful than is mortality. This does not apply to cases where mortality forms the barrier effect. The well-being of individuals is discriminated against the long term viability of entire populations. While the barrier function ultimately refers to a situation, in which the vast majority of individuals of a 
species does not cross a road, it also includes conditions where movements dissimilar to the need of dispersion are significantly restricted, so that the fitness of a population is not harmed by missing genetic exchange, but by non-satisfactorily met demands for instance in foraging or visiting seasonal habitats. Both dimensions of impact are considered under the subsequent method of quantification, with impeded dispersion being considered to be slightly more severe. Hence, a road representing a complete barrier is weighted as more detrimental than other yet substantial disjunctive effects, which can also render a population extinct, but are not as likely to do so when assuming a longterm perspective. However, a case in which a population directly is driven extinct due to the denial of access to essential resources is weighted as severe as a complete barrier, also because an extinct population cannot contribute to genetic exchange. In a first step, several thresholds of traffic volume were determined at which structural changes and intensification of fragmenting impact is assumed. Table 5.18 shows these thresholds and specifies the increase in negative impact.

Table 5.18: Thresholds of daily traffic volume (dtv) of substantially increasing fragmenting impact (see Charry \& Jones 2009, Forman \& Sperling 2011, and also Seiler 2005, Gibbs \& Shriver 2002, Gibbs \& Shriver 2005, Glista et al. 2008; see the original graphic of Charry \& Jones 2009 in Appendix D).

\begin{tabular}{|c|c|}
\hline Thresholds & Form of fragmenting impact \\
\hline$\sim 2,000 \mathrm{dtv}$ & $\begin{array}{l}\text { - first changes in rates of animal movements and begin- } \\
\text { ning of severe impacts from road mortality for some } \\
\text { species, i.e. reptiles }\end{array}$ \\
\hline$\sim 3,000 \mathrm{dtv}$ & - first substantial impacts on ungulates and carnivores \\
\hline$\sim 5,000 \mathrm{dtv}$ & - increases in mortality rates for most species \\
\hline$\sim 10,000 \mathrm{dtv}$ & $\begin{array}{l}\text { road represents a near complete barrier for most spe- } \\
\text { cies }\end{array}$ \\
\hline$\sim 30,000 \mathrm{dtv}$ & $\begin{array}{l}\text { - road is a complete barrier to all ground bound and } \\
\text { some aerial species }\end{array}$ \\
\hline
\end{tabular}

In a second step, different degrees of severity are assigned to the dissimilar fragmenting impacts produced by different traffic volumes. Factors are allocated in order not only to rank the impacts of dissimilar daily traffic volumes but continuously to relate the impacts to each other by their degrees of severity. The possible consequences of impacts were described in Table 5.18. In the previous paragraph, insights on the criteria by which the severity of impact was assessed and which weighting of factors this would lead to, were given. The factor weights assumed for dissimilar daily traffic volumes are indicated in Table 5.19. 
Table 5.19: Relating the fragmenting impact of dissimilar traffic volumes by factor weights.

\begin{tabular}{|cc|}
\hline Daily traffic volume & Factor weights \\
\hline 2,000 & 0.8 \\
3,000 & 1.0 \\
5,000 & 2.0 \\
10,000 & 5.0 \\
30,000 & 10.0 \\
50,000 & 10.0 \\
90,000 & 10.0 \\
\hline
\end{tabular}

Since the function that best describes the relation of the pairs of variates is a logarithmic formula, it can only be used from 5,000 vehicles per day onwards until up to 150,000 vehicles per day or further (see Equation 5.16a). For daily traffic volumes between 500 and 5,000 vehicles, Equation 5.16b well represents the relation of the factor weights. For roads with traffic volumes below 500 vehicles, traffic volume shall be assumed to be at 500 vehicles per day in order not to complicate matters by adding another formula that well represents the impact of traffic volumes at this lowest range.

Equations 5.16: Mathematical functions calculating factors of fragmenting impact subject to traffic volume.
a) $f(d t v)=2.8498 \ln (d t v)-21.317 \quad$ for $\mathrm{dtv} \geq 5,000$
b) $f(d t v)=0.009816 d t v^{0.6701} \quad$ for $500 \leq d t v<5,000$

It applies to the ranges of validity of both functions that the factor weight of fragmenting impact increases with every additional vehicle. This means that while the factor weights suggested in Table 5.19 express the assumption of no additional impact being produced by traffic volumes higher than 30,000 vehicles per day, the resulting equation for daily traffic volumes at or above 5,000 vehicles does yield increasing factors of impact also in the range between 30.000 and 200,000 vehicles. The reason to assume a maximal impact at 30,000 vehicles was that a road represents a complete barrier for most species at this level of traffic. However, as there probably are some few species that considerably suffer from the barrier effect only at even higher traffic volumes and as the increase in impact per additional vehicle, particularly from a level of about 58,250 vehicles onwards, when an impact factor of 10 is yielded (see Table 5.20, column 2), becomes very small because the logarithmic function begins to level off and as the remaining increase is outweighed by the effect of proportioning the impact between a larger number of users, it is viewed suitable to have an ongoing increase of impact also for very high traffic volumes. In Table 5.20, the here mentioned development of factor 
weights as well as values of fragmenting impact obtained from using the final formula as of Equation 5.17 to calculate the result for an individual heavy duty vehicle using a road for 10 kilometers at various hypothetical traffic volumes, are indicated.

With regard to the formula referring to traffic volumes below 5,000 vehicles per day it is to be said that no minimum quantity of vehicles was assumed in order for the barrier function beginning to be effective. While it can be argued that particularly for levels of traffic below 2,000 vehicles per day, only very few species are meaningfully affected by the barrier effect, some studies found that low use roads or even decommissioned gravel or tarred roads alone, can significantly reduce animal abundance near those roads, leading to an implied contribution to fragmentation (Semlitsch et al. 2007, Marsh \& Beckman 2004). As already a change of surface or of vegetation creates unfavorable conditions and a reduction of free movement for some species, it was assumed for a fragmenting impact to be present from the lowest numbers of users onwards. Same as for the calculation of a fragmenting value assuming a corridor-orientated approach, vehicle factors originally determined in section 5.1.1, which are not skewed in favor of the criterion of noise, are used. Thus, Table 5.17 can be turned to to obtain the vehicle factors $f\left(v_{x}\right)$ of dissimilar vehicle types. Hence, in order to calculate a value of fragmenting impact of an individual user driving a particular route that accounts for traffic volume and vehicle size, assuming a discretionary landscape approach $\left(F^{d l a}\right)$ the formula depicted in Equation 5.17 can be used.

Equation 5.17: Quantification of the fragmenting impact of an individual road user when applying a discretionary landscape orientated assessment of impact.

$\mathrm{FV}_{\mathrm{dla}}=\sum_{\mathrm{i}=1}^{\mathrm{n}} \frac{d_{i} \cdot \mathrm{f}\left(d t v_{i}\right)=\left\{\begin{array}{c}2,8498 \ln \left(d t v_{i}\right)-21,317 \quad \text { for all } d t v \geq 5,000 \\ 0.009816 d t v_{i}^{0,6701} \quad \text { for all } 500<d t v<5,000\end{array}\right\}}{\mathrm{dtv}_{i} \cdot(1.1379)} \cdot \mathrm{f}\left(\mathrm{v}_{\mathrm{x}}\right)$

$\mathrm{FV}_{\mathrm{dla}} \quad=$ fragmenting value using a discretionary landscape approach

$\mathrm{d}_{\mathrm{i}} \quad=$ length of section $\mathrm{i}$

$\mathrm{i}=$ homogenous section $\mathrm{i}$, with $\mathrm{i}=1,2, \ldots, \mathrm{n}$ (consistent in traffic volume)

$\mathrm{dtv}_{\mathrm{i}}=$ number of vehicles/ day in section $\mathrm{i}$ with dtv to equal 500 for all $\mathrm{dtv}<500$

$f\left(v_{x}\right) \quad=$ vehicle factor of vehicle type $v_{x}$ as of Table 5.17

Besides vehicle type, traffic volume is the main variable to determine total fragmenting impact and to distribute shares of impact among users. At the center of the formula, the impact factor of a section of uniform traffic volume, which is the result obtained from Equation 5.16, is multiplied by the length of that section. The result is divided by the sum of passenger car units present in that section in one day. This represents a deviation from the two indicators on land use, where impact was split among all users within 
one year. However, fragmenting impact more directly is produced by and thus to be split among the total of users of a 24-hour period. Next, the total of the results of all sections, which is that of one PCU, is multiplied by the vehicle factor of the vehicle of concern. The result can be multiplied by the quantity of trips per year to obtain a yearly total. Table 5.20 exemplarily indicates fragmenting values of a heavy duty vehicle $\left(v_{t<12}\right)$ driving in one section for 10 kilometers, assuming a variety of potential traffic volumes.

Table 5.20: Factor weights and values of fragmenting impact calculated for a heavy duty vehicle $\left(\mathrm{v}_{\mathrm{t}<12}\right)$ driving a distance of $10 \mathrm{~km}$ for various traffic volumes.

\begin{tabular}{|rcc|}
\hline $\begin{array}{c}\text { Daily traffic } \\
\text { volume }\end{array}$ & $\begin{array}{c}\text { Factor } \\
\text { weights }\end{array}$ & $\begin{array}{c}\text { Value of fragmenting } \\
\text { impact per user }\end{array}$ \\
\hline 500 & 0.6317 & 33.31 \\
1,000 & 1.0052 & 26.50 \\
2,000 & 1.5994 & 21.08 \\
3,000 & 2.0987 & 18.44 \\
5,000 & 2.9554 & 15.58 \\
10,000 & 4.9360 & 12,00 \\
20,000 & 6.9060 & 9.10 \\
30,000 & 8.0615 & 7.09 \\
50,000 & 9.5172 & 5.02 \\
70,000 & 10.4761 & 3.95 \\
100,000 & 11.4925 & 3.03 \\
150,000 & 12.6480 & 2.22 \\
\hline
\end{tabular}

Comparing the resulting values of fragmenting impact, the differences seem appropriate. Also they incentivize users to use higher traffic roads. For instance, using a road with a daily traffic volume of 50,000 vehicles is a little over four times more efficient than using a road with only 2,000 vehicles per day. While this speaks in favor of using higher use roads when possible, at an aggregate level, fragmentation will only decrease if traffic shifts toward larger roads. Obtaining better individual values by increasing traffic on all roads of a road grid, only the individual user is better off while the total impact aggravates. Multiplying the impact of one PCU by the total of PCUs according to traffic volume, the aggregate development of a selected road can be monitored.

While for a corporate entity, the results calculated following a corridor- and habitatorientated approach can best be lowered by reducing haulage distance, achieving a higher rate of vehicle utilization, and choosing a different route, under the discretionary landscape approach the major triggers for improvement are the usage of more efficient higher traffic roads and the reduction of haulage distances. Also rates of vehicle utilization remain as a variable for improvement, but are not expected to hold much potential because they for long have been attended to for monetary reasons. 


\subsection{Application of the Indicators}

The potential implementation of the indicators is tested by exemplarily calculating the results for two transportation routes. The first is from Hann. Münden to Hannover to Brunswick and back to Hann. Münden, all cities being located in Lower Saxony, Germany, and the second is from Hannover to Wendelstein, Bavaria (see Appendix E for maps). The frequency of the first transport is 40 trips per year, the second is assumed to take place more frequently, namely 1.4 times per week on average, which equals 73 trips per year. In both cases, the vehicle used is a semitrailer tractor. Sources of data for the courses of the routes, section lengths, and traffic volumes, are the ones indicated in the previous sections. Tables 5.21 and 5.22 show the results obtained for both routes.

Table 5.21: Impact of the route Hann.Münden, Hannover, Brunswick, to Hann.Münden [route 1]; distance of the trip: $354.5 \mathrm{~km}$; frequency of the trip: 40 trips per annum.

\begin{tabular}{|c|c|c|c|}
\hline Type of impact & ... per trip & $\begin{array}{l}\text { Impact ... } \\
\text {... per year }\end{array}$ & $\ldots$ per $1 \mathrm{~km}$ \\
\hline SSA (in $\mathrm{m}^{2}$ ) & 2.92 & 116.72 & 0.0082 \\
\hline NDLI (in $\left.\mathrm{m}^{2}\right)$ & 126.82 & $5,072.77$ & 0.3577 \\
\hline $\mathrm{FV}_{\text {cha }}$ & 302.00 & $12,080.00$ & 0.8519 \\
\hline $\mathrm{FV}_{\mathrm{dla}}$ & 293.80 & $11,752.03$ & 0.8288 \\
\hline
\end{tabular}

Table 5.22: Impact of the route Hannover (Lower Saxony) to Wendelstein (Bavaria) [route 2]; distance of the trip: $479.9 \mathrm{~km}$; frequency of the trip: 73 trips per annum.

\begin{tabular}{|c|c|c|c|}
\hline Type of impact & ... per trip & $\begin{array}{l}\text { Impact ... } \\
\text {... per year }\end{array}$ & $\ldots$ per $1 \mathrm{~km}$ \\
\hline SSA (in $\left.\mathrm{m}^{2}\right)$ & 3.09 & 225.80 & 0.0064 \\
\hline NDLI (in $\mathrm{m}^{2}$ ) & 156.49 & $11,423.96$ & 0.3261 \\
\hline $\mathrm{FV}_{\text {cha }}$ & 533.00 & $38,909.00$ & 1.1106 \\
\hline $\mathrm{FV}_{\mathrm{dlla}}$ & 318.11 & $23,222.18$ & 0.6629 \\
\hline
\end{tabular}

Legend: $\quad \mathrm{SSA}=$ share of sealed area; $\mathrm{NDLI}=$ noise driven landscape impact; $\mathrm{FV}_{\text {cha }}=$ fragmenting value using a corridor- and habitat-orientated approach; $\mathrm{FV}_{\mathrm{dla}}=$ fragmenting value using a discretionary landscape-orientated approach.

Regarding route 2 from Hannover to Wendelstein, the fragmenting value determined using a corridor- and habitat-orientated approach ( $\left.F V_{\text {cha }}\right)$ is slightly inaccurate because 
no information on locations of conflict for wet and dry corridors and habitats for the area of the federal state of Hesse is available. While in $128 \mathrm{~km}$ or $26.5 \%$, this concerns a significant portion of the route, compared to the fragmenting value of forest and forest living mammal habitats, the relevance of dry habitats and corridors is relatively weaker because in Germany they are more rare. This applies to wet habitats to a much lower degree. As information on fragmenting impacts regarding corridors in Hesse is available for forest and forest living species habitats, the reliability of the method of quantification is viewed to be satisfactory for all routes, whose share of route driven in Hesse is $50 \%$ at most.

In the following analysis, two subjects are concerned: firstly, to test the possible ecological superiority of alternative routes as indicated by the four impacts of concern, and secondly, to compare the results per average kilometer of each route to each other in order to learn more about the characteristics and sensivities of the formulae.

With regard to the first route, which is a round trip, studying the course of the route only one leg of the route seems reasonable to be chosen differently. That is the very first and very last part, leaving from and coming back to Hann. Münden. Instead of going slightly south on B80 and then going strictly north on $A 7$, it is possible right away to go north on B3 from the industrial zone of origin and to access A7 at a farther northern point at Göttingen (see maps in Appendix E). While this option is $10 \mathrm{~km}$ shorter, the leg is 20 instead of $30 \mathrm{~km}$ long, the share of sealed area is more than twice the amount of the longer, but more area efficient alternative (see Table 5.23 for numeric results), the noise driven landscape impact is only slightly, namely $12 \%$ lower, and while also the fragmenting value of the discretionary landscape approach sets the more userintensive, longer alternative better off (the impact value is 35.6 compared to 49), only the fragmenting value of the corridor- and habitat-orientated approach yields a significantly better result for the alternative route (1 to 5.75). The reason for the latter is that the original route runs through the important forest habitat corridor "Kaufunger Wald". This situation illustrates well, why an approach that complements the corridororientated approach is useful. Not calculating $F V_{\text {dla }}$, the result of $F_{\text {cha }}$ would have indicated to avoid using the road that runs through the important forest corridor. While this might still be the superior option, this verdict at least is questioned by the results of the discretionary landscape approach, which stresses the presence of fragmenting impact produced by lower use roads. Table 5.23 summarizes the values of the original leg and the alternative, lower use leg. While the results suggest no clear preference, it seems that overall the original route is preferable due using a more efficient high use road. This applies in particular, if a solution to re-establish connectivity for the forest corridor Kaufunger Wald can be found. 
Table 5.23: Comparison of the original and an alternative leg as part of an analysis targeted to mitigate spatial and fragmenting ecological impact.

\begin{tabular}{|r|cc|}
\hline Type of impact & Current route on B80 and A7 & Alternative route on B3 \\
\hline $\mathrm{SSA}\left(\right.$ in $\left.\mathrm{m}^{2}\right)$ & 0.31 & 0.82 \\
$\mathrm{NDLI}\left(\right.$ in $\left.\mathrm{m}^{2}\right)$ & 12.85 & 11.20 \\
$\mathrm{FV}_{\text {cha }}$ & 5.75 & 1.00 \\
$\mathrm{FV}_{\mathrm{dla}}$ & 35.6 & 49.00 \\
\hline
\end{tabular}

With regard to the second route, two possible alternative legs are tested for their potential ecological superiority as of the four impacts of concern. The first option would be to go via Bamberg, that is to exit A7 further north at Schweinfurt and to take A70 to go east and later to take $A 73$ to go south to Nuremberg (see the map displayed in Figure 5.13). While the distance of the alternative route is almost the same, 112 instead of $110 \mathrm{~km}$, and while no significant difference in the amount and severity of locations of fragmenting conflict as considered under the corridor- and habitat-orientated approach is found, a meaningful difference shows in the intensities of use of the two routes. Here the firstly assumed route via $A 7$ and $A 3$ has significantly higher traffic volumes than the alternative route via $A 70$ and $A 73$, particularly when comparing $A 70$ to $A 3$ for the westeast axis. As the three directly traffic-related formulae SSA, NDLI, and FV $\mathrm{V}_{\text {dla }}$, yield lower values of individual impact with rising traffic volumes, without calculating detailed results it can be concluded that the original leg is preferable with regard to the ecological impacts of concern.

The same reasoning, and here to an even greater degree, applies to the second alternative leg, which is not to continue on A3, but to drive via Neustadt, exiting A3 at Wiesentheid and taking federal highways B286 and B8 to Nuremberg. While the distance is marginally lower, namely 78 compared to $81 \mathrm{~km}$, and while the alternative leg avoids dissecting the high priority corridors of the Steigerwald, which is both habitat and corridor for forest living mammals, and the wet Aisch River habitats, the much lower intensity of use of both federal highways B286 and B8 compared to the originally used freeways dominates to argue in favor of the firstly assumed leg via A3 and A73 when it comes to overall spatial and fragmenting ecological impact. This concludes the analysis of possible alternative courses that reasonably could have been used had they been found to mitigate the ecological impacts considered by the indicators developed in this work. 


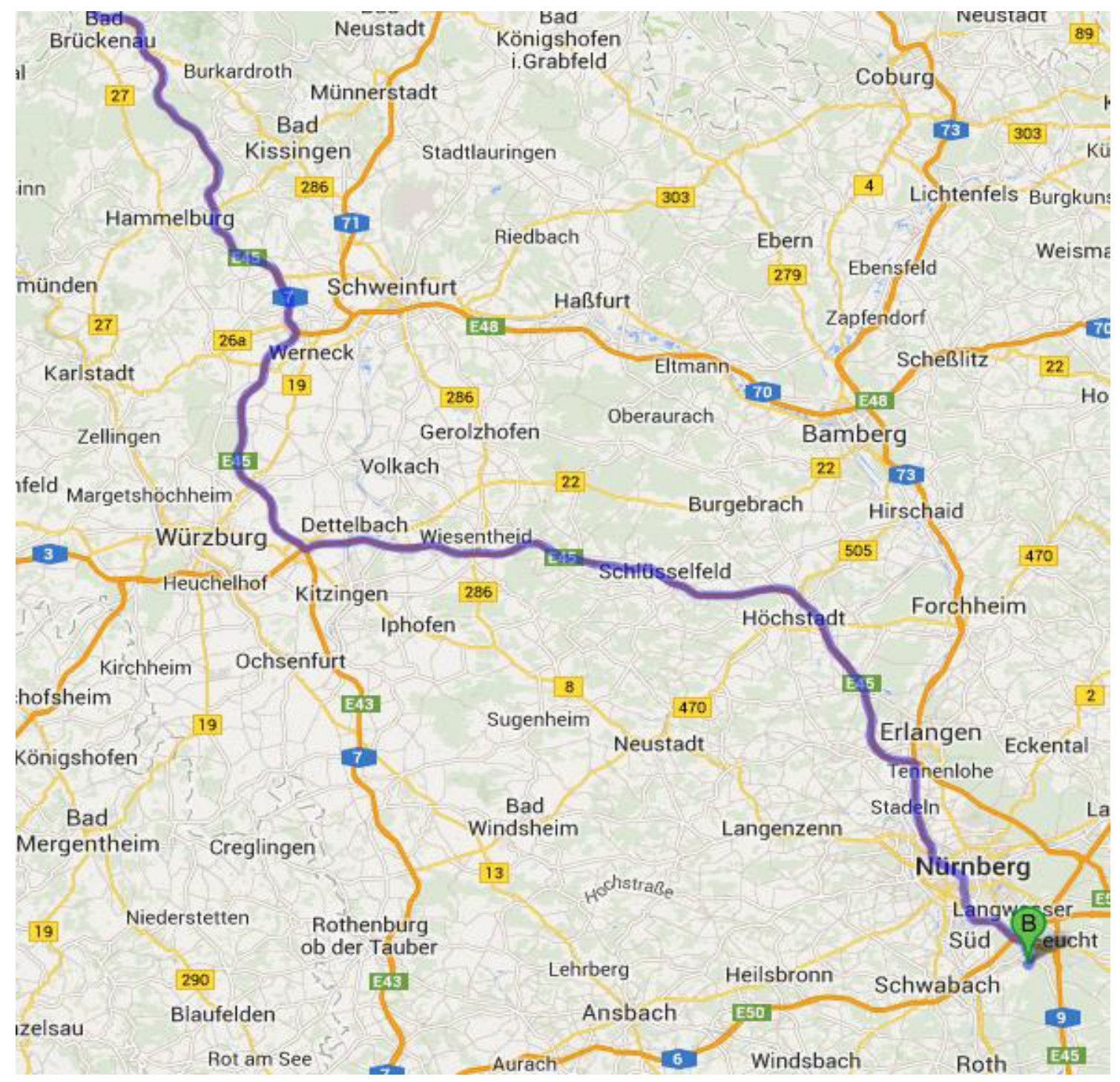

Figure 5.13: Display of the final part of route 2; source: Google Maps 2014a.

In a second to last step of the analysis of the exemplary results, the magnitude of impact of the average kilometer driven on the two routes is compared (see columns 4 of Table 5.21 and 5.22). For the share of sealed area, route 2 yields a significantly lower kilometric value, namely $64 \mathrm{~cm}^{2} / \mathrm{km}$ compared to $82 \mathrm{~cm}^{2} / \mathrm{km}$. This is caused both by the differing intensities of use and the differing shares of smaller roads of the two routes. While the average kilometric traffic volume is 54,416 for route 2 it is 50,502 for route 1 . In addition, the percentage of smaller roads, which usually have a much worse traffic volume to sealed area ratio, is only $0.85 \%$ for route 2 , but is $4.24 \%$ for route 1 . This results in a significant difference of kilometric SSA-values of the two routes; with route 2 having the notably better ratio of sealed area per kilometer travelled. That the noise driven landscape impact implies a much more linear relation between traffic volume and degrading impact shows in the more moderately deviating kilometric results of 
$\mathrm{NDLI}$ for the two routes, which are $0.3261 \mathrm{~km}^{2} / \mathrm{km}$ for route 2 and $0.3577 \mathrm{~km} / \mathrm{km}$ for route 1 . The fragmenting values obtained when applying a corridor- and habitatorientated approach are more difficult to compare because they depend on landscape individual geographies to a high degree. Furthermore, it shall be recalled that the $\mathrm{FV}_{\text {cha }}$ of route 2 is not accurate because information on locations of conflict was missing for about one quarter of the route. However, the overall lower $\mathrm{FV}_{\text {cha-result of route } 1 \text {, which }}$ is 0.8519 compared to 1.1106 , can be explained for the geographies because route 1 in Hannover and Brunswick runs through metropolitan, less natural areas to a larger share than does route 2 . Finally, the large difference in fragmenting values using a discretionary landscape orientated approach between routes 1 and 2 again goes back to the higher efficiency of use of larger roads. $F_{\mathrm{dla}}$ being 0.6629 for route 2 and 0.8288 for route 1 shows that the $\mathrm{FV}_{\mathrm{dla}}$-formula accounts for this gradual characteristic, which was built into the formula in spite of possible cases where absolute thresholds of fragmenting impact of traffic volumes exist. Overall, the differing results yield no surprise as they can be reasoned and they are an indication of the functionality and reliability of the formulae.

In a last step of verification, the results obtained for the share of sealed area of an individual vehicle are compared to national meta-data. According to the data from the Federal Statistical Office, area under the category 'Road, Path, and Place' in Germany in 2012 covered 15,708 km² (Federal Statistical Office 2012). Subtracting the area that is not used for motorized road bound mobility, roughly $15,318 \mathrm{~km}^{2}$ remain. Dividing this number by the total national motorized mileage of 690.1 million kilometers yields an average value of $0.0222 \mathrm{~m}^{2}$ or $222 \mathrm{~cm}^{2}$ per one vehicle kilometer. This number is three to four times the size of the numbers of the exemplary kilometric results of the SSAformula discussed above. Taking into consideration that the roads of the above calculation are federal highways and freeways at large, which are the most sealed area efficient ones, the deviation from the gross average area value per vehicle kilometer does not shed doubt on the quality and reliability of the here developed formula and even can be seen to support their functionality.

\subsection{Remaining Limitations of the Indicators}

While remaining limitations were already referred to when developing the respective formula, they shall be summarized in order for potential users to be able to obtain a concise overview on the remaining weaknesses. This is viewed helpful for users who may want to apply the formulae and for researchers and practitioners who may assess and consider developing the formulae further. Moreover, it will be pointed out, which variables are likely to change over time and hence will have to be adapted in the future. 
Two subjects that concern all four formulae are the assumed vehicle factors and the missing compilation of all necessary data in one database. With regard to vehicle factors, two different sets of factors were assumed. The factors used to determine the share of sealed area (SSA) and both fragmenting values ( $\mathrm{FV}_{\mathrm{cha}}$ and $\mathrm{FV}_{\mathrm{dla}}$ ) were adopted and merged from earlier transport studies, namely Delft et al. (2011) and ProgTrans AG \& IWW (2007). In the studies, the factors focused on the areal and material demands of different types of vehicles on the road surface. They did not explicitly include environmental impact, such as emissions, although the correlation between the variables that determined the factors and relative environmental impact is positive. Due to the spatial relevance, it was viewed appropriate to use the factors for the SSA-formula and for the quantification of fragmenting impact. Concerning the latter, their suitability is most uncertain. The vehicle factors were considered to be inappropriate to be used for the noise dominated landscape impact. With regard to the adjusted factors that were then used in the NDLI-formula, the depth of investigation and argumentation is assumed to have produced dependable factors, which nonetheless can be questioned. With regard to the conditions of availability of the data, at this early stage of development of the indicators it was viewed sufficient to refer to and to describe the data and not to process the data to be available in a more convenient format. It is beneficial that all necessary data is freely available.

Two limitations that concern the calculation of the individual share of sealed area, are expected changes of road sizes in the future and the inaccuracy of the SSA-formula with regard to roads smaller than federal highways and freeways. Despite the introduction of new guidelines for the design of motorways and country-roads, cross-sections and road width are expected only to change slowly over time and even then are expected not to change by much because the difference between the former and the new guidelines is marginal and because no drastic changes in traffic volumes or regulation for instance on speed limits are foreseen. However, the missing compatibility of the formula to smaller roads is a significant constraint. While the average haulage distance in 2000 was $84 \mathrm{~km}$ and in 2010 it was $104 \mathrm{~km}$ and hence is an indication that the share of utilization of larger roads like federal highways and freeways dominates at this distance, the numbers also show that far from all road bound transports are of long distance. In addition, the trend of longer haulage distances on roads may attenuate, if rail and water infrastructure are improved, subsidized, and used for modal split to a higher degree. Statistics however speak against such a development (UBA 2012). The more relevant point is that roads are not just used for long distance import/ export transports or nationwide shipments from production facilities to warehouses and customers, but that a significant share falls back to local and regional product distribution, a share that the current SSA-formula cannot adequately consider. Due to the difficulty to use the 
highly individual data of the large variety of traffic volumes and medium and smaller roads, averages that yield less accurate values are likely to have to be used. Resulting values are assumed still to be helpful in certain contexts.

The indicator developed to quantify degrading impact on adjacent area is abbreviated NDLI, which stands for noise driven landscape impact. Potential imperfections of the method lie in the inclusion of urban area as a receiver of habitat degrading impact, the assumption of national averages of noise extension inflexible to consider sites individually, and the dominance of the effect of noise over other impacts like emissions, road mortality, or fragmentation, the spatial impacts of which are not adequately represented. With regard to the inclusion of urban area, a distinction between area of settlement and industrial area may be suitable due to their distinct predispositions in being locations of living for humans and non-human species. In addition, it seems that more research on the assumption of $55 \mathrm{~dB}(\mathrm{~A})$ to be an appropriate threshold of disturbing impact is needed and that the data sample used under the practical approach should be enlarged to more states and potentially to more topographies, if the precautionary approach of sampling mainly open landscapes is dismissed.

Four limitations apply to the calculation of a fragmenting value using a corridor- and habitat-orientated approach. Three of them concern the underlying work of identifying, weighting, and mapping the considered fragmenting conflicts of Hänel and Reck (2011). In the study, conflicts are assessed and assigned to five groups of different priorities. In addition, there exists a sixth group entitled 'not further classified, subordinated conflicts'. Further research on the entries of this group would improve the quality of the data. Similarly, the second weakness, which consists of the lack of data of wet and dry habitats and corridors for the state of Hesse, could be remediated. The third limitation is that while the research did cover four major types of habitat, it insufficiently considers aquatic species and hydrologic systems. A fourth limitation may lie in the weighting of the points of conflict assumed in this work.

In addition to the disputable suitability of vehicle factors referred to above, for the calculation of a fragmenting value using a discretionary landscape approach, the assumed fragmenting impact of dissimilar traffic volumes as of the two intermediary equations should be subject of further discussion. Both the magnitude of impact as well as the assumed procedure to begin attributing a fragmenting impact from the first vehicle on and not to set a maximum threshold should be challenged. Another limitation is the inclusion of urban area, because under $\mathrm{FV}_{\mathrm{dla}}$ fragmenting impact is assumed to occur for the entire route. While it can be reasoned to include urban area in the calculation of noise driven landscape impact, it seems inappropriate to assume a fragmenting impact for areas where less species or species nonsensitive to noise are living. Nonetheless, 
urban biodiversity does exist, for instance in parks, gardens, or allotments. Thus, further research on this trade-off is necessary to reconsider the decision to omit or to include urban areas when referring to the fragmenting impact of roads.

Two limitations that concern the two methods of quantification of fragmenting impact and the formula on degrading impact are the needs for more qualitative studies that investigate the effects of road traffic on the long term viability of adjacent populations, communities, and ecosystems. One focus should be on the development and severity of the impacts of genetic differentiation and depletion produced by fragmentation. Thereto, complex and expensive molecular genetic approaches need to be applied to a higher degree. Concerning the $\mathrm{FV}_{\mathrm{cha}}$ and $\mathrm{FV}_{\mathrm{dla}}$-methods of quantification of fragmenting impact in particular, research urgently needs to investigate the effectiveness of wildlife crossing structures at the population level, because their construction is one central feature of current efforts of defragmentation.

Moreover, regarding the two methods of quantification of fragmenting impact, a difficulty that can arise from using two approaches to assess the same impact, is the possibility to obtain contradicting results. While both indicators have the same generic, numeric measuring unit, it is not possible to sum both results to obtain a total because issues of weighting were tackled within each indicator, but did not account for the comparability and relation of the two. That the values of $\mathrm{FV}_{\text {cha }}$ and $\mathrm{FV}_{\text {dla }}$ in the exemplary calculation of route 1 are nearly identical and neither are far apart for route 2 , wrongly suggests their direct comparability. While calibrating the indicators, so that an identical resulting value would mean an equal severity of impact, could be an undertaking to attend to in the future, it was not intuitive to be pursued here and it would not be free of disadvantages. The main reason is that the underlying philosophies of the two approaches of measurement, which are either to focus on priorities of conflict determined from a to some degree utilitarian and anthropocentric perspective or to assume responsibility for the impact irrespective of the location and the quantity of species and individuals concerned, prove to be of significant difference. However, as long as they are not merged inextricably into one indicator, calibration of the indicators would still allow users to value and weight one approach higher than the other, in spite of the suggested equivalence implied in their calibration. The situation of having two indicators may be beneficial because it can foster discussion and understanding of the impact of fragmentation and can contribute to meaningful standardization.

The final constraint to be mentioned is the limited global immediate applicability of two of the four the indicators. While the NDLI-formula on degraded area adjacent to roads and the $\mathrm{FV}_{\mathrm{dla}}$-formula on fragmenting impact that pursues a discretionary landscape approach have universal validity, the SSA-formula on sealed area and the $\mathrm{FV}_{\text {cha- }}$ - 
formula on fragmenting impact when pursuing a corridor- and habitat-orientated approach in this context hold limitations both in precision and applicability, because they use data that is likely to be specific to Germany or that may not be available in other countries. This concerns the measurement of the share of sealed area to a mild degree, because although road categories and yielded average lane widths per category are expected to vary between countries, lane width as such and the overall correlation between road size and average lane width are expected to be similar in most countries. Thus, the indicator can be used in other countries to obtain approximate results. In order to obtain precise results, present road categories have to be determined and their respective average lane widths have to be recalculated. Likely, it is not possible directly to apply the $\mathrm{FV}_{\text {cha }}$-formula in other countries because it uses the results of an extensive study carried out for Germany. However, if similar studies are available, the uncomplicated methodology can be readily adapted. Although all four indicators and their underlying assumptions may have to be changed to meet the specific ecological conditions and the cultural valuation of impacts of specific regions and societies, the studies and findings used for their development came from all over the world, so that a certain degree of global validity is ensured.

\subsection{Summary}

In this section it is summarized briefly, how the four indicators that allow quantifying land use, habitat degradation, and fragmentation, are developed, what parameters they consist of, and which limitations remain in their application. In section 5.3, exemplary results are calculated and discussed. The methods of quantification are targeted to measure the spatial impacts produced by traffic on larger roads, namely federal highways and freeways. However, they can also be used to account for the impacts of traffic on smaller roads, because the criterion of road type is not as important as is average daily traffic volume. Only calculating the share of sealed area, road type is important. Hence, the SSA-formula yields particularly precise results for higher use roads. Thus, in order to obtain highly accurate results using the SSA-formula, the percentage of roads that are smaller than federal highways or that carry less than 1,000 vehicles per day should be below $10 \%$ for a given route. For the indicators on habitat degradation and fragmentation, results are reliable for traffic volumes as low as circa 1,000 vehicles per day. Applying them, the share of roads with less than 1,000 vehicles per day should not surpass $10 \%$.

In order to determine the share of sealed area that an individual road user is responsible for, five variables for all sections of a route need to be known. As the total area sealed by a road and its supporting infrastructure like resting areas and bypasses is 
determined by the number of lanes and the average lane width of a road of a certain category, road category (freeway $=\mathrm{FW}$, federal highway $=\mathrm{FH}$, or smaller road $=\mathrm{SR}$ ) and the quantity of lanes $\left(\mathrm{l}_{\mathrm{i}}\right)$ of each section need to be known. In addition, information on daily traffic volume $\left(\mathrm{dtv}_{\mathrm{i}}\right)$ has to be obtained, not only because it is used to calculate the share of impact of an individual user, but also because traffic volume, number of lanes, and road type are the three variables that divide a route into individual sections. The assumption is that one section ends and another begins whenever one of the three variables changes. The length of each section is another variable that needs to be identified $\left(\mathrm{d}_{\mathrm{iFW}}, \mathrm{d}_{\mathrm{iFH}}, \mathrm{d}_{\mathrm{iSR}}\right)$. Accounting for differing impacts between different types of vehicles, the vehicle type $\left(v_{x}\right)$ of a vehicle being used has to be known in order to retrieve the respective vehicle factor $\left(f\left(v_{x}\right)\right)$. With this information the formulae indicated under Equations 5.3 can be used to determine the share of sealed area of an individual user. The unit of measurement of the result is square meters.

A second type of spatial impact that the analysis in Chapter 3 found to be undervalued and often to be left out completely is the degradation of area adjacent to roads. Several individual impacts like tangible and intangible emissions, invasive species, mortality, or the barrier effect of a road together produce the overall effect of changing and usually degrading plant and animal species' habitats. Despite the effect of noise is concluded to be the spatially most significant direct impact, the method of quantification developed here does account for several of the other impacts as well. Noise is hence used for the calculation of widths of road-effect zones. $55 \mathrm{~dB}(\mathrm{~A})$ is assumed as the threshold of disturbing impact. Two approaches are followed to attempt establishing an elementary mathematical formula that reliably represents the correlation between traffic volume and the spreading of noise, so that the share of degrading impact of a user can be measured from traffic volume. The first approach resorts to the formulae used by governmental institutions to monitor the expansion of sound pressure level in order to cope with legislation. Despite the rate of attenuation being assumed as $3.5 \mathrm{~dB}(\mathrm{~A})$ per doubling of distance is viewed to yield realistic expressions (see Equations 5.7d and e), the formula resulting from the second approach, which used a data sample of noise zone extensions and traffic volumes, is regarded to be more reliable. It constitutes a part of the more complex expression that calculates the noise driven landscape impact (NDLI) of an individual user. As noise zone extension depends on average daily traffic volume, traffic volume is the parameter to divide a route into individual sections. The length of each section $\left(\mathrm{d}_{\mathrm{i}}\right)$, is the second variable that needs to be known. The intermediary result is multiplied by two in order to account for the extension of noise on both sides of a road. In order to obtain the impact of one individual user, the resulting total areal value is divided by the total impact of all users within a year. In a last step, vehicle type is accounted for. Vehicle factors $\left(f\left(v_{x}\right)\right)$ differ from those assumed for the indicator on 
sealed area because the generation of sound pressure level relates to the same variables differently and to a few additional variables. The completed formula that allows quantifying the noise driven landscape impact per vehicle per route is listed under Equation 5.14. Again, the unit of measurement of the result is square meters.

Landscape fragmentation is the third spatial ecological impact that two quantifiable, complementary indicators are developed for. Since both knowledge on the constitution of the impact, referring to its formation and the consequences on species and ecosystems, and societal valuation of the impact, showing in degrees of discussion and the states of national targets, are not completed to a meaningful degree, the indicators are chosen not to state the 'impact', but to express a fragmenting value. Further, and for the same reasons, two approaches that differentiate in their underlying philosophies, either prioritizing locations of impact or accounting for the impact irrespective of the magnitude of impact, were followed. The first approach largely builds on a work of Hänel and Reck (2011), in which habitats and corridors of four different habitat types, wet, dry, forest, and forest living large mammal habitats, were identified for all of Germany and which in a second step were compared to the national grid of water, rail, and road infrastructure in order to define locations of fragmenting conflict where species corridors and transport infrastructure intersected. In a third step, the locations of conflict were assessed for the severity of the individual conflict, which depended on the ecological importance of a habitat or corridor, on the severity of the barrier function of an infrastructure, and on the presence or absence of opportunities for wildlife to cross an infrastructure, so that in the end maps and lists were created that grouped the conflicts by their severity. This fulfilled the central research goal, which was to determine priorities of abatement. Under the method of quantification presented here, the conflicts present in a given route are multiplied by numeric values ranging from 5 to 0.25 in order to account for their dissimilar severity. The result is multiplied by a vehicle factor, which is chosen to be the same as in the calculation of the share of sealed area of an individual user. The result is a numeric value that allows for a first estimation of fragmenting impact of a route and that can be used to compare alternative routes or the impacts of transport activities between years. The indicator is specified under Equation 5.15.

The complementing second approach of estimating the magnitude of fragmenting impact of road use applies a discretionary landscape-orientated assessment of impact. This means that all locations of a route are treated equally, not prioritizing one over another. The reasoning behind this is that roads at a large range of traffic volumes impede the movement of animals and plants throughout the entire landscape, be it that in some locations fewer species and individuals are concerned than in others. As already a change of surface or of vegetation represents an obstacle to some species, a fragmenting impact is assumed to be present from low numbers of road users onwards. 
Fragmenting impact is assumed to increase with rising traffic volume, with the effect of an additional vehicle being particularly significant up to daily traffic volumes between 20,000 and 30,000 vehicles, because at this threshold the last large group of species is viewed to be included considerably to be affected by the barrier effect. The formula that represents these characteristics is logarithmic. To obtain an equation, different severities of impact are assigned to various traffic volumes. The function that is obtained does not well represent impacts below 5,000 vehicles per day. Therefore, a second function covering the range from 500 to 5,000 vehicles is built. As for the indicators on land use, average daily traffic volume divides a route into individual sections. The results of all sections have to be added and multiplied by the same vehicle factors used under the complementing indicator of fragmentation in order to obtain a route's total fragmenting value. Thus, the three variables of the $\mathrm{FV}_{\mathrm{dla}}$-indicator are daily traffic volume $\left(\mathrm{dtv}_{\mathrm{i}}\right)$, distance travelled per section $\left(\mathrm{d}_{\mathrm{i}}\right)$, and a vehicle factor $\left(f\left(v_{x}\right)\right)$. Overall, assuming a discretionary landscape approach, the formula depicted under Equation 5.17 can be used to calculate a value of fragmenting impact of an individual user driving a particular route. The result takes the form of a numeric value.

In section 5.3, the indicators are tested by calculating results for two exemplary routes. One is a 355 kilometer long round trip in Lower Saxony and the second route runs across a large part of Germany, covering 480 kilometers going north to south, from Hannover to Wendelstein near Nuremberg. Results yield a share of sealed area of 2.92 $\mathrm{m}^{2}$ for route 1 and $3.09 \mathrm{~m}^{2}$ for route 2 . The area adjacent to the roads that is degraded predominantly by noise amounts to $127 \mathrm{~m}^{2}$ for route 1 and $156 \mathrm{~m}^{2}$ for route 2 . The fragmenting values of the corridor- and habitat-orientated approach are 302 for route 1 and 533 for route 2. Applying the complementary discretionary landscape approach, fragmenting values are 294 for route 1 and 318 for route 2 . The impression is that the results display realistic values. Testing both routes for potential superior courses regarding spatial impact and fragmentation, the situation that in both cases higher use roads are chosen over lower use alternatives, produced the result that no alternative courses are found that can improve the above mentioned results. This is positive because it means that the methods function in favor of the general opinion that concentrating traffic on fewer, higher use roads is environmentally preferable to spreading traffic more evenly across the landscape. The comparison of the two routes enhances this quality because route 2 yields superior kilometric values for SSA and FV dla, which is ascribed to route 2 having a higher average kilometric traffic volume than route 1. The less significant difference in NDLI is evidence of the fact that traffic volume and landscape impact correlate positively. That route 2 yields a higher result for $F V_{\text {cha }}$ explains because it runs through less built-up area and hence has a higher probability of 
producing fragmenting conflict than route 1. Overall, the results yield no surprise and are an indication of the functionality and reliability of the indicators.

With regard to the limitations indicated in section 5.4, the main constraint in the calculation of the share of sealed area (SSA) is the inaccuracy of results for smaller roads and the resulting inability precisely to measure regional transports. Determining the share of degraded area adjacent to a road, which is measured as noise driven landscape impact (NDLI), the inclusion of adjacent built-up area, the assumption of a noise disturbance threshold at $55 \mathrm{~dB}(\mathrm{~A})$, and the moderate degree of ability to account for other extending ecological impacts like emissions other than noise, changes in water regimes, erosion, sedimentation, mortality, the barrier effect of roads, or even the insertion of invasive alien species, are three subjects that require further discussion. Regarding the quantification of fragmenting impact produced by an individual vehicle being driven on a certain route, when assuming a corridor- and habitat-orientated approach ( $F V_{\text {cha }}$ ), the missing data on locations of conflict in the state of Hesse, the existence of remaining, not further classified conflicts for all four habitat types, and the weighting of conflicts as of five groups of priority plus a sixth group of remaining conflicts, appear as deficiencies of the method. Approximating a calculation of fragmenting impact when applying a discretionary landscape-orientated approach $\left(\mathrm{FV}_{\mathrm{dla}}\right)$, the weighting of impact of dissimilar traffic volumes implied in the two corresponding equations should be questioned and discussed. Secondly, the inclusion of urban area under this indicator is disputable and hence needs to be researched and discussed further. Finally, two constraints that concern all four indicators are, firstly, the assumption of the vehicle factors, which were not found yet to have been used in environmental contexts, and secondly, the missing compilation of all necessary input data in one database. Conclusions that concern the results and the levers of mitigation at the individual corporate and at the national economic level, as well as a discussion of the advancement of the research context and applied organizational environmental management practices are at the center of the following, second to last chapter of this work. 


\section{Research Conclusion and Outlook}

Despite some of the environmental impacts of transport are heard and felt in our immediate environment and thus are more visible than are many other impacts that occur at different stages of supply chains and in often significantly displaced locations, the impacts of road transport are largely disregarded in corporate environmental management, in consumers' awareness, and in political agendas. Possible reasons are that at a time of global sourcing and distribution, the cost of the first or last few hundred haulage kilometers is still very important and that owning and using a car became normality for most people, so that others could not be pointed to for engaging in intensive road use. Moreover, although in society and politics a consensus on the undesirability of human induced climate change developed in recent years, taxes on fuel remained unchanged. Likely, all three reasons, economic competitiveness, adapted and enjoyed mobility intensive life styles, and the difficulty to investigate the diffuse ecological impact of roads and traffic on species and ecosystems, played a part in having overlooked environmental impacts of road transport to a notable degree. Knowledge of the impacts concerned by the indicators developed in this work and of their levers for mitigation yields the result that a slight improvement of existing processes through better environmental management, is insufficient to abate impacts to a degree that would allow speaking of strongly sustainable practices of production, distribution and consumption to be established. Despite the possibilities to ship more area-efficiently by stressing freight consolidation or by using different modes of transport, or to mitigate impacts by improving technology (e.g. quieter road surfaces), by adapting behavior (e.g. speed or route selection) or by building wildlife crossings or (vegetated) berms, for the three impacts of land use, land degradation, and fragmentation, the obtained improvements are marginal compared to the effect that could be obtained from lowering transport intensity in production and distribution. To this end, haulage distances could be reduced for instance by using regional resources, by allowing larger lead times or by reducing shipments to farther away customers (cp. Aronsson \& Brodin 2006, Wu \& Dunn 1995). This is consistent with the principle that, regarding a problem alone, a problem is most effectively abated by being avoided compared to being mitigated. This holds particularly true for the ecological impacts of roads and traffic, because the functionality of several measures of mitigation is not fully understood and hence uncertain, and because in some cases solving one problem has the consequence of aggravating another. If the leveling rule was strong sustainability, a reduction of haulage distances and of overall mobility intensity would be needed.

While this larger, macro-economic outcome is rather plain, it does not render superfluous the development of indicators of this work. The methods of measurement allow for a better understanding of the impacts and they allow determining the concrete individ- 
ual size of impact. While not requisite, these two factors facilitate reflecting on the desirability of and responsibility for the impacts and they support their management. The research outcome relates to business administration since in order to organize production and distribution efficiently, guardrails and criteria of efficiency need to be known. While efficiency is viewed to be a positive theme in business administration, other tenets like the generation of consumerist needs, the acceptance of disrespectful occupation and of significantly unequal distribution of capital resources, the overemphasis of the human being as being egocentric, independent, and rivaling, and, recapitulatory, the exemption of ethics from economics, as criticized prior by Thoreau or Fromm, for instance leading to the oblivion of questioning the ends of personal and collective economic activity like happiness or self-development and as thoroughly done by ancient ethicists like Aristotle or Seneca or leading to an insufficient query of responsibility in complex conditions (Cafaro 2009a, 2009b, Fromm 1976), are viewed to be harmful.

Independent of an individual goal, business administration builds around efficiency. Analyses, concepts, and calculations target to support corporate decision-making in order to achieve a favorable ratio of inputs and outputs. Input resources are assumed to be scarce. Resources can take any form; the most prominent ones are materials, labor, time, and finances. Under the ancient ideal of the honorable merchant, consequences on closer or further society and on the environment are considered and their presumed interests are not violated (Klink 2008), which means to include them as matters of scarcity, and that with a very high weighting. Today, it seems that the larger projects and enterprises grew, and the further life cycle consequences reached, the less were fellow human beings' and non-human nature's interests considered and the more was regulation regarded sufficiently to represent their interests. However, due to augmenting welfare in well-off, industrialized countries and due to increasing understanding of ecological deterioration, environmental impacts have again gained in importance at the beginning of the $21^{\text {st }}$ century. This makes it necessary to include environmental impact into the criteria catalogue of decision making and to attribute it meaningful weight. This research advances business administration because knowledge and calculation of so far neglected environmental impacts move forward existing management of ecological impacts and improve the basis for efficient decision making. The indicators allow companies to compare alternative routes, vehicles and strategies of sourcing and delivery on a unit basis at a point as well as over the course of time.

Despite being regarded fit for application, the formulae presented and the approaches chosen for their development should be criticized and improved. This applies to the list of limitations indicated in section 5.4 and to the overall biological context, which includes the uncertainty and difficulty to understand which species are affected by the effects of road transport, what the consequences of impacts and changed behaviors 
are for affected and subsequently affected species, and what the potential impacts on the functioning of local and regional ecosystems are. Uncertainty about systemic and lagging implications of impacts in particular renders the description and quantification of effects difficult. Present knowledge in chemistry allowed determining quantities of greenhouse gas emissions of certain activities fairly accurately. Consequences of an impact on biological diversity, which implies more than the presence or absence of a species but also concerns the abundance of individuals, population fitness, or ecosystem stability, prove to be more difficult to measure and to estimate. While the first method of measurement of the individual impact on sealed soil is rather straightforward and the margin of error is limited, the implied question, where, how much area can be sealed and habitat be taken away without forfeiting ecosystem processes to an undesirable amount or without menacing ecosystem diversity is more difficult to answer. With regard to the other three indicators of impact, this holds true to a higher degree because the impacts of degrading seminatural area mainly through noise and of impeding biotic and abiotic movements by roads and traffic are less clear than is the sealing of soil. Nonetheless, evidence of the impacts was viewed to have been found and presented by respective studies in sufficient quantity and quality, as to use the results to represent the effects of wider landscape impact and fragmentation by the here developed indicators. In addition, due to the multitude of species concerned and due to the unclear valuation of individual fates and of non-human nature in general, information will not and does not have to be complete and definite to begin considering the impacts at hand. It shall be emphasized that the quantification of impacts is no curtate accusation of lorry use, because through consumptive decisions most people approve of it. Approximate quantification is a possibility to become aware and to evaluate, to report, to manage, and to consider the impacts in corporate daily or strategic decisions, or, at longer sight, as awareness and valuation develop, in consumptive choices.

With regard to policy instruments, numerous options to mitigate and to incentivize the avoidance of impacts exist. They range from more uncontroversial measures like

- the construction of quieter road surfaces,

- the construction of sound attenuation barriers, or

- the construction of different types of crossing structures,

to not necessarily monetarily, but more behaviorally impacting measures like

- supporting more area efficient modes of transport,

- establishing larger undissected low-traffic areas by regulating through traffic and rural traffic in general (here, a toll on major roads alone or route navigation systems that by trend spread traffic, are disadvantageous), 
- supporting the longevity of products, or

- supporting regional economic cycles, e.g. by imposing higher taxes on imports and exports, by increasing prices of transport, or by assisting regional currencies and personal and regional activities of subsistence.

Particularly measures that are directed at reducing haulage distances and gross national mobility intensity while allowing for equal or increasing welfare, inter alia are faced by one structural and one eudemonic constraint, which seem closely related: firstly, that is the material growth paradigm, which provokes peoples' alienation from each other and the earth, and which forms the understanding that material occupancy is most apt to feel freedom, security, self-actualization and happiness, and secondly, the unattractiveness of simplicity and sufficiency as virtues or elements of a good life. While a thorough ethical discussion on whether lowered mobility intensity is desirable is needed, overcoming these two constraints seems to have the potential for people unsolicitedly to reconsider their values and to live more self-determined and more sustainable within meaningful moral and ecological boundaries. For many to welcome such change, a reconnection with the land and with all, which comes from encounter and not from knowledge, is necessary. Regarding logistics, it then needed to be analyzed, which demands of a partially regionalized economy transport processes would have to meet and how reduced environmental impact could be ensured.

With regard to the limitations of the presented indicators, in addition to the weaknesses and restrictions inherent to the methods referred to in section 5.4, four ambient topics need further research: firstly, the indicators presented here can only be used for road transport. Research on and measurement of the impacts produced by other modes of transport is still missing. Secondly, despite having been determined to be the most significant undervalued impacts besides greenhouse gas emissions, land use and fragmentation do not complete the count of ecological impacts of road transport as for instance the problem of invasive alien species remains. Thirdly, this work focused on the relevance of the impacts for non-human nature. The consequences on humans, which regarding health risks are known to greater detail, should also be considered. Finally, it is mentioned that while significant parts of landscapes in industrialized countries are used for intensive agriculture, which means that noise and the barrier effect do not apply to as many species as in seminatural locations of high biodiversity, in this work no differentiation between the ecological qualities of landscapes is made. This is because even in intensively agriculturally used zones some landscape components like trees of shrubs remain, rendering the respective habitats particularly important. Moreover, enabling biological diversity is a service and a goal of increasing importance in agriculture. Thus, as long as intensively used zones are not formed and not set apart, it is regarded inappropriate to discount certain types of seminatural landscapes. 


\section{Summary}

Throughout the world land use and fragmentation are among the major causes of the destruction of habitats and the resulting loss of biological diversity. Besides climate change, the loss of biological diversity is the second environmental meta-crisis humans are provoking and facing. Due to biodiversity's high complexity in meaning and morale and a missing corporate inclination to assume responsibility for adverse environmental impacts, the adaption and instrumentalization of biodiversity in environmental management still are at an early stage. Analyzing corporate efforts, and tools used in management and reporting of ecological impacts from transport, incomplete practices of environmental management that are often limited to address greenhouse gas emissions were found (Chapter 3 ). To establish a more complete list of impacts, direct land use, which refers to the sealing of soil and the impact on adjacent area, and landscape fragmentation were conceived as the most important impacts, next to be assimilated by corporate environmental management (Chapter 4). To this end, this work presents four methods of quantification, one concerning sealed area, a second considering the degrading impact on adjacent area, and two others referring to the impact of fragmentation. The suggested methods shall stimulate the development of standardized indicators and they can facilitate the immediate consideration and management of these impacts in practice (Chapter 5). In addition, opportunities for mitigation at the companyand policy-level, and a reflection of the nature and the limitations of the formulae, are given (Chapter 6). In the following, the courses of action and the main findings of the five principal chapters of this text are summarized. In the second part of this summary, the four articles that were written during the time of research are briefly referred to. It further is explained, how the articles and their findings relate to the central theme of developing methods of quantification for the impacts of land use and fragmentation.

In Chapter 2, the context of the research question is analyzed. In the beginning, scientific findings of the ecological conditions and the state of political awareness regarding increasingly threatened biological diversity are indicated. The second part of the chapter deals with the question, why biodiversity is valued by humans. The capabilities approach by Nussbaum and the sustainable person by Becker are two concepts that take further the affirming reasons of tangible and intangible benefits. In section 2.3, the perception of sustainability in society and five structural constraints of economic organization that the implementation of sustainability is facing, are addressed. These are the inefficient conditions of external effects and of non-regulated, high market complexity, decreasing consumer and increasing capital sovereignty, and material growth. Mainly for the finiteness of "natural resources" and for the recognition that rising material wealth only at low levels is a significant component of happiness, the growth paradigm is rejected by the economic concept of degrowth, which is introduced. 
In Chapter 3, the status of consideration of biodiversity and ecological impacts of road transport in corporate environmental management is analyzed. Neither the most complete organizational management tool, nor the most proactive companies are found to consider either theme appropriately. The findings of this chapter produce the original research question, which is to work on a more complete recognition and management of environmental impact for transportation. For the relevance of road transport, and for the significance of the impacts of land use and fragmentation, in Chapter 4 the characteristics and consequences of the effects of road mortality, habitat loss and degradation, and fragmentation, are focused on. The negative consequences that the three effects have in common are reduced population size and higher risk of extinction. The fragmenting impact of the barrier effect of a road, which means the blocking of animals from resources, habitats, and mates, appears to be the most harmful impact because it can reach as far as to structurally weaken species' genetics. It stands as a result of Chapters 3 and 4 that for their underrepresentation in environmental management and for their relevance in disturbing and threatening individuals, species, and ecosystems, the goal of the research will be to develop methods of quantification of the impacts of sealed area, degraded area, and fragmentation in order to facilitate their inclusion into environmental management. The indicators that were built are explained in Chapter 5.

In section 5.1, two formulae to measure immediate land use and degradation of adjacent area are developed. To determine the share of sealed area SSA (see Equation 5.3) of an individual road user, five variables need to be known. These are average daily traffic volume $\left(\mathrm{dtv}_{\mathrm{i}}\right)$, road type ( $\left.\mathrm{FW}, \mathrm{FH}, \mathrm{SR}\right)$, number of lanes $\left(\mathrm{l}_{\mathrm{i}}\right)$, section lengths $\left(d_{i}\right)$ and vehicle type $\left(v_{x}\right)$. Constructing a formula to quantify extending degrading impact, noise is assumed as the dominant and spatially most significant impact. A level of $55 \mathrm{~dB}(\mathrm{~A})$ is assumed as the lowermost threshold of disturbing impact. Applying a data based approach, the formula that was obtained expresses the correlation of traffic volume and sound pressure level (see Equation 5.14). Average daily traffic volume $\left(\mathrm{dtv}_{\mathrm{i}}\right)$, section lengths $\left(d_{i}\right)$, and vehicle type $\left(v_{x}\right)$ are the required variables. Same as for the share of sealed area, the unit of measurement of the result is square meters. In section 5.2 , two complementing methods to measure the fragmenting impact of a vehicle driving a certain route are presented. Under the corridor- and habitat-orientated approach $\mathrm{FV}_{\text {cha, }}$, prioritized locations of conflicts are weighted and added for an entire route (see Equation 5.15). Conflict maps of different habitat types $(h)$, and vehicle type $\left(v_{x}\right)$ are the necessary input data. Under the discretionary landscape orientated approach $F V_{\text {dla }}$, all locations of the landscape are treated as of equal importance. As fragmenting impact is assumed to correlate with traffic volume, for the calculation, daily traffic volume $\left(d t v_{i}\right)$, section lengths $\left(d_{i}\right)$, and vehicle type $\left(v_{x}\right)$ need to be known (see Equation 5.17). In section 5.3 , the formulae are tested by calculating results for two exemplary transports. 
Results confirm the higher ecological efficiency of larger roads and attest the reliability of the formulae. In section 5.4, lasting limitations of the formulae are depicted.

In final Chapter 6, research conclusions are drawn and further outlook is given. It is concluded that while freight consolidation and usage of alternative modes of transport offer potential to mitigate ecological impacts of transport, a reduction of haulage distances is the most effective lever. Yet, this strategy requires strong commitment from a company and it is difficult to realize because it would benefit from multi-dimensional change, for instance in economic and individual aspirations. Despite this finding, the research at hand is viewed to advance business administration because the developed indicators raise awareness and allow for a better understanding of impacts, and, allowing to determine a concrete individual size of impact, facilitate potential management and mitigation. The indicators should be questioned in order to strengthen or add to their reliability in measuring the impacts of land use and fragmentation, particularly with regard to the complex context of ecosystem affectedness and biodiversity. Still, results of the available studies were regarded to be sufficiently informative and reliable to construct the presented formulae. Regarding possible regulatory policies, options to mitigate the impacts are limited and levers of lowering gross mobility intensity are unattractive also to government. Chapter 6 is concluded by making reference to potential limitations of the indicators. Moreover, several themes from the ambient context of quantification of environmental impacts from road transport that need further research, such as the impacts of other modes of transport, further effects of road transport itself, or the relevance of the impacts investigated here for humans, are described.

In continuation, brief summaries of the four articles written on the topic of research are given. They are reprinted succeeding the appendix of this document. Article I is entitled "Potential impacts of road haulage on biodiversity". The text was written in German and the original title is "Potentielle Auswirkungen von Straßenverkehrstransporten auf die Biodiversität". The text identifies land use, including degradation, fragmentation, road mortality, substance emissions, invasive alien species, and life-cycle impacts of roads and vehicles as six significant ecological impacts of roads and traffic. This list falls short to include the impacts of erosion, sedimentation, and changes in hydrology. Toward the end, rudimentary approaches that allow measuring the impact per road user for land use and fragmentation are introduced. The text was relevant for the development of the formulae because the overview of all existing impacts allowed making a first assessment of the significance of the individual impacts. This led to the selection of land use and fragmentation as the two impacts that should be attempted to be quantified.

Article II is entitled "Current practices of mitigating the ecologically harmful effects of roads - An assessment". Building on the political proposition to reduce and to suspend 
adverse effects of road transport on biological diversity, in this article recent conditions and trends of the effects of land use, material emissions, noise and light, and fragmentation are analyzed. The focus is on the discussion of the two major strategies of defragmentation currently applied and debated in science and practice. They can be described as a corridor-centered and an area-centered strategy. The latter has the advantage that maintaining and re-establishing large non-dissected low traffic areas is viewed to be most effective in supporting ecosystem stability and the conservation of biodiversity. Large crossing structures are a central feature of a corridor-centered approach. They are assumed to solve the problem of fragmentation only for a certain number of species in certain places. The advantage of the approach is that despite high cost of construction, the overall cost to society can be perceived as acceptable because no changes in economic organization and lifestyles are necessary as levels of mobility and land use intensity can persist. As both strategies have to complement each other, the goal of the discussion is to contribute to finding their 'right' weighting. Regarding other impairments, the positive trend in lowered land use is too weak to meet set goals, a further reduction of material emissions remains necessary, and more measurements and research are needed to track and to tackle the development of the impacts of light and noise. Findings of the article proved relevant for the development of the indicators because they evoked the conception that fragmenting impact at this early stage of discussion should be represented by both a more mitigation driven approach and a more conservative, non-biasing approach.

The third article is entitled "Traffic induced landscape fragmentation in the Biobio Region of Chile: Analysis and prospects". In this text, results of an analysis of the degree of fragmentation in the state Biobío are presented. The motivation for the analysis was that in regions with high road densities costly and uncertain measures of mitigation are applied in order to restore a certain degree of landscape permeability, and that for regions with increasing transport intensities the most effective and most feasible approach would be to be aware of the problem in order to be able to avoid it before it establishes. Having dissimilar strengths and weaknesses, the three methods of undissected low traffic areas, effective mesh size, and relation perimeter/ area were applied to determine the degree of fragmentation. Results reveal that the overall level of fragmentation in the Biobío Region is moderate. While disturbances and threats to individual animals exist, the up-keeping of minimum viable population sizes is assumed to rarely be impeded. Genetic variability across the landscape might be negatively affected. It is viewed suitable both to apply strategies of avoidance, such as bundling traffic or supporting sustainable resource use, and to employ strategies of mitigation in respective, already severely dissected locations of ecological importance. The simultaneously developed indicators on fragmentation benefit from the large landscape-level 
analysis because it built understanding on the complexity of the impact of fragmentation and on its relevance in different landscape contexts.

The fourth article is entitled "Integrating neglected ecological impacts of road transport into corporate management". Its content is an abridged form of this text. The focus is on the presentation of the four methods of quantification that were explained in detail on the previous pages. The methodology of the development of the indicators measuring the share of sealed area and fragmentation is literature analysis and it is data analysis for the indicator measuring the share of degrading impact on adjacent area. Two exemplary case studies are described in order to illustrate the characteristics of the formulae and of the results. The methods of quantification are intended to allow businesses to measure the impacts produced by their road transport activity. Results can be used in the context of LCA and for reporting, management, and mitigation.

To conclude, in this work methods that allow measuring the share of degraded area adjacent to roads and the impact of fragmentation produced by an individual vehicle are proposed for the first time. Also, the quantification of the share of sealed area is more precise than currently applied approaches that calculate individual impact from gross national data. The method presented here takes into account the parameters of traffic volume and size of a particular road. The analysis of the depth and integrity of environmental management tools together with the study of a multiplicity of ecological impacts caused by road use produced the research task of advancing and pioneering the quantification of the impacts of land use and fragmentation. The objective is to provide knowledge to corporate staff and decision-makers and to facilitate the inclusion and management of the referred to impacts in environmental management. Not least for the need of the pioneering methods to be challenged and advanced, the work is also directed at practitioners working on environmental management tools and guidelines, and at researches working in fields as diverse as biology or business economics.

Finally it shall be remarked that at this point in history extra indicators that usefully increase the allocation of responsibility appear insufficient to solve the environmental crises formed by humans across the world. The indicators shall be used carefully because quantitative relationships today are dominating human lives, despite the essence of humans' social lives lies in qualitative rather than in quantitative relationships, it is moral, not technological (Gregg 2009). Ethical reflection of the human/ non-human nature relation as well as reflection of the quality of currently dominating economic and political organization and agenda to support all humans to live dignified and flourishing lives, today and I daresay in the future, appear to be two of the more important matters people living today may do good to commit themselves to. 



\section{References}

Adidas AG. 2013. 2012 Sustainability Progress Report - Never Stop . Herzogenaurach: Adidas AG.

Agent, K.R., and Ch.V. Zegeer. 1981. Propagation of Traffic noise. Research Report UKTRP-81-21. Kentucky: Transportation Research Board.

Alexander, S. 2009. Voluntary simpllicity - the poetic alternative to consumer culture . Whanganui: Stead and Daughters Ltd.

Alexander, S.M., N.M. Walters, and P.C. Paquet. 2005. Traffic volume and highway permeability for a mammalian community in the Canadian Rocky Mountains. The Canadian Geographer 49(4): 321-331.

Ancestry. 2009. Map of German Federal States. Provo. Http://www.google.de/imgres?client=firefoxa\&hs=qr0\&sa=X\&rls=org.mozilla\%3Ade\%3Aofficial\&biw=1440\&bih=730\&tbm=i sch\&tbnid=NT8PIVJdB_VuAM\%3A\&imgrefurl=http\%3A\%2F\%2Ffamilytreemake r.genealogy.com\%2Fusers\%2Fs\%2Fe\%2Fv\%2FSarah-J-

Severs\%2FPHOTO\%2F0004photo.html\&docid=TLKD6iEzEbX-

3M\&imgurl=http\%3A\%2F\%2Ffamilytreemaker.genealogy.com\%2Fusers\%2Fs\% 2Fe\%2Fv\%2FSarah-J-Sev-

ers\%2FPHOTO\%2F0004photo.jpg \&w=308\&h=414\&ei=cRLhUvOfEoS24wTAyl CQBQ\&zoom $=1$ \&iact=rc\&dur $=314 \&$ page $=1 \&$ start $=0 \&$ ndsp $=36 \& v e d=0 C G A Q r Q$ MwAw (accessed 23 January 2014).

Andel, P., T. Minarikova, and M. Andreas. 2010. Protection of landscape connectivity for large mammals . Liberec: Everina.

Aristotle. Politics . 1282b 20.

Aronsson, H., and M.H. Brodin. 2006. The environmental impact of changing logistics structures. The International Journal of Logistics Management 17(3): 394-415.

Aulig, G., R. Bachfischer, J. David, and H. Kiemstedt. 1978. Wissenschaftliches Gutachten zu ökologischen Planungsgrundlagen im Verdichtungsraum NürnbergFürth-Erlangen-Schwabach . Munich: Selbstverlag Lehrstuhl für Raumforschung, Raumordnung, Landesplanung TU München.

Babisch, W. 2003. Stress hormones in the research on cardiovascular effects of noise. Noise Health 5: 1-11.

Balkenhol, N., and L.P. Waits. 2009. Molecular road ecology: exploring the potential of genetics for investigating transportation impacts on wildlife. Molecular Ecology 18: 4151-4164.

Barber, J.R., K.R. Crooks, and K.M. Fristrup. 2010. The costs of chronic noise exposure for terrestrial organisms. Trends in Ecology and Evolution 25(3): 100-109. 
Bartolomaeus, W. 2010. Comparison of road noise emission models. Lärmbekämpfung 5(2010): 179-187.

Bartolomaeus, W. 2013. Personal communication. December 13, 2013, Göttingen, Bergisch Gladbach.

Bast (Federal Highway Research Institute). 2005. Survey of kilometers travelled in 2002 by vehicles registered in Germany . Bergisch Gladbach: Bast.

Http://bast.opus.hbz-nrw.de/frontdoor.php?source_opus=199\&la=de (accessed 23 January 2014).

Bast (Federal Highway Research Institute). 2007. Autobahnverzeichnis 2006. In Berichte der Bundesanstalt für Straßenwesen , eds. Bundesanstalt für Straßenwesen, pp. 1-219. Bergisch Gladbach: Bundesanstalt für Straßenwesen.

Http://bast.opus.hbz-nrw.de/frontdoor.php?source_opus=165\&la=de (accessed 23 January 2014).

Bast (Federal Highway Research Institute). 2011a. Manual Traffic Census 2010 - Results on Federal Highways . Bergisch Gladbach: Bast

Http://www.bast.de/cln_030/nn_42248/DE/Statistik/Verkehrsdaten-

Downloads/2010/Manuelle-Zaēhlung-2010.html (accessed 23 January 2014).

Bast (Federal Highway Research Institute). 2011b. Manual Traffic Census 2010 - Results on Freeways . Bergisch Gladbach: Bast.

Http://www.bast.de/cln_030/nn_42248/DE/Statistik/Verkehrsdaten-

Downloads/2010/Manuelle-Zaehlung-2010.html (accessed 23 January 2014).

Bast (Federal Highway Research Institute). 2014. Bauwerke an Straßen als bestehende Querungsmöglichkeiten aus dem Bundesinformationssystem Straße (BISStra) und der Datenbank "Querungshilfen" . Bergisch Gladbach: Bast.

Baudrillard, J. 2005. Nique ta mère. Http://www.liberation.fr/tribune/2005/11/18/niqueta-mere_539377 (accessed 18 March 2014).

Bauer, J. 2013. VAUDE - Auf dem Weg zum nachhaltigsten Outdoor Ausrüster Europas . Presentation at Göttingen University, 15 November 2013.

Baumert, K.A., T. Herzog, and J. Pershing. 2005. Navigating the Numbers. Greenhouse Gas Data and International Climate Policy. Washington, DC: World Resource Institute.

Bechmann, A., and J. Hartlik. 2004. Die Bewertung zur Umweltverträglichkeitsprüfung ein methodischer Leitfaden . Barsinghausen: Verlag Edition Zukunft.

Bechmann, A. 1998. Zur Bewertung für die Umweltverträglichkeitsprüfung - Wie zeitgemäß ist die ökologische Risikoanalyse? . SYNÖK-Report 21, Barsinghausen: Verlag Edition Zukunft.

Becker, Ch. 2010. Sustainability ethics, Pennsylvania: STS Program and Department of Philosophy, Pennsylvania State University. 
Belardi, M., G. Catullo, Ch. Massacesi, R. Nigro, P. Padoan, and Ch. Walzer. 2011. Webs of life. Alpine biodiversity needs ecological connectivity. Results from the ECONNECT project . Cava de' Tirreni Salerno: Grafica Metelliana.

Benítez-López, A., R. Alkemade, and P.A. Verweij. 2010. The impacts of roads and other infrastructure on mammal and bird populations: a meta-analysis. Biological Conservation 143: 1307-1316.

Bertiller, R., C. Schwick, and J.A.G. Jaeger. 2007. Landschaftszerschneidung Schweiz. Zerschneidungsanalyse 1885 - 2002 und Folgerungen für die Verkehrs- und Raumplanung. Bern: ASTRA-Bericht.

BfN (Federal Agency for Nature Conservation). 2009. Rote Liste der Tiere, Pflanzen und Pilze Deutschland 2009ff. From 2009. Rote Liste gefährdeter Tiere, Pflanzen und Pilze Deutschlands. Band 1: Wirbeltiere, eds. H. Haupt, G. Ludwig, H. Gruttke, M. Binot-Hafke, C. Otto, and A. Pauly. Naturschutz und Biologische Vielfalt 70(1): 1-386.

BfN (Federal Agency for Nature Conservation). 2013. Ermittlung der Unzerschnittenen Verkehrsarmen Räume $>100$ km² für Deutschland 2010 . Leipzig: BfN.

BfU (Bavarian Agency for Environment). 2014. Cadaster of Noise Exposure. Munich. Http://www.bis.bayern.de/bis/initParams.do (accessed 23 January 2014)

Biglin, K., and L.-A. Dupigny-Giroux. 2006. Mapping the road-effect zone to assess impacts of proposed road segments. Journal of Conservation Planning 2: 1-16.

BImSchG 16 (Bundes-Immissionsschutzgesetz). 1974. Gesetz zum Schutz vor schädlichen Umwelteinwirkungen durch Luftverunreinigungen, Geräusche, Erschütterungen und ähnliche Vorgänge. Http://www.gesetze-iminternet.de/bundesrecht/bimschg/gesamt.pdf (accessed 11 March 2014).

Bissonette, J.A., and W. Adair. 2007. Restoring habitat permeability to roaded landscapes with isometriacally-scaled wildlife crossings. Biological Conservation 141: 482-488.

Bitom. 2004. Noise. Breisach-Oberrimsingen.

Http://www.bitom.de/Problem/Larm/larm.html (accessed 29 January 2014)

BKG (Federal Agency for Cartography and Geodesy). 2006. Digital Basic Landscape Model - Bauwerke an Straßen zur Auswahl bestehender Querungsmöglichkeiten; bebaute Flächen (Barrieren) als Grundlage zum Aufbau des Netzwerkes der Wälder . Frankfurt am Main: BKG.

BKG (Federal Agency for Cartography and Geodesy). 2008. Digital Landscape Model 250 - Bauwerke an Straßen zur Auswahl bestehender Querungsmöglichkeiten; bebaute Flächen (Barrieren) als Grundlage zum Aufbau des Netzwerkes der Wälder. Frankfurt am Main: BKG.

Bladen, V.W., and J.M. Robson (eds.). 1965. The Collected Works of John Stuart Mill, Principles of political economy . Toronto: University of Toronto Press. 
Blickley, J.L., and G.L. Patricelli. 2010. Impacts of Anthropogenic Noise on Wildlife: Research Priorities for the Development of Standards and Mitigation. Journal of International Wildlife Law \& Policy 13: 274-292.

BMU (Federal Ministry for the Environment, Nature Conservation and Nuclear Safety). 2011. National strategy on biological diversity . $3^{\text {rd }}$ edition. Berlin: BMU.

BMU (Federal Ministry for the Environment, Nature Conservation and Nuclear Safety). 2010. Der Indikatorenbericht 2010 zur Nationalen Strategie zur biologischen Vielfalt . Eichenau: Schottenheim druck\&werbung.

BMU (Federal Ministry for the Environment, Nature Conservation and Nuclear Safety). 1998. Mit dem Entwurf eines umweltpolitischen Schwerpunktprogrammes neue Etappe in der Umweltpolitik eingeleitet . Bonn: BMU-Pressedienst.

BMVBS (Federal Ministry of Transport, Building and Urban Development). 2011. Statistics of length of roads of overland traffic as of January 2011 (Längenstatistik der Straßen des überörtlichen Verkehrs, Stand: 1. Januar 2011). Berlin: BMVBS.

BMVBS (Federal Ministry of Transport, Building and Urban Development). 2014. Güterverkehr und Logistik (Freight haulage and logistics). Berlin: BMVBS. Available online at Http://www.bmvi.de/SharedDocs/DE/Artikel/IR/gueterverkehr-undlogistik.html.

BMVBS (Federal Ministry of Transport, Building and Urban Development). 2012a. $A$ Map of Highways in Germany (Bundesfernstrassen in Deutschland) . Bonn: BMVBS.

BMVBS (Federal Ministry of Transport, Building and Urban Development). 2012b. Modernisierung der BVWP-Methodik, Teil "Städtebauliche Effekte", Schlussbericht. . Berlin: BMVBS.

Http://www.bmvbs.de/SharedDocs/DE/Anlage/VerkehrUndMobilitaet/bvwp2015-modernisierung-methodik-staedtebau.pdf?_blob=publicationFile (accessed 17 December 2013).

BMW Group. 2013. Umwelterklärung BMW Group . Munich: BMW.

Boarman, W.I., and M. Sazaki. 2006. A highway's road-effect zone for desert tortoises (Gopherus agassizii). Journal of Arid Environments 65: 94-101.

Bolt Beranek and Newman, Inc. 1973. Fundamentals and abatement of traffic noise. Report No. FH-WA-HH1-HEV-73-7976-1. Washington, DC: National Highway Institute.

Brand, U. 2012. Growth and authority (Wachstum und Herrschaft). Aus Politik und Zeitgeschichte 62(27-28/2012): 8-14.

BRD (Federal Republic of Germany). 1990. Gesetz über die Umweltverträglichkeitsprüfung (UVPG) (Law on Environmental Impact Assessment). Http://www.gesetzeim-internet.de/bundesrecht/uvpg/gesamt.pdf (accessed 13 January 2014). 
Brumm, H., and H. Slabbekoorn. 2005. Acoustic communication in noise. Advances in the Study of Behavior 35: 151-209.

Brumm, H., K. Voss, I. Köllmer, and D. Todt. 2004. Acoustic communication in noise: regulation of call characteristics in a New World monkey. The Journal of Experimental Biology 207: 443-448.

Burch, M. 2009. Why simplify? In Voluntary simpllicity - the poetic alternative to consumer culture, eds. S. Alexander, pp. 181-205. Whanganui: Stead and Daughters Ltd.

Business \& Biodiversity Campaign. 2011a. Biodiversity Management. Radolfzell, Germany. 2011. Http://www.business-biodiversity.eu/default.asp?Menue=23 (accessed 24 September 2011).

Business \& Biodiversity Campaign. 2011b. Biodiversity Check. Radolfzell, Germany, 2011. Http://www.business-biodiversity.eu/default.asp?Menue=128 (accessed on 24 September 2011).

Business in Good Company. 2011. Online Handbook Corporate Biodiversity Management - Checklists. Business in Good Company; Berlin, Germany, 2011. Http://www.business-and-biodiversity.de/en/handbook/checklists.html\#c5233 (accessed 24 September 2011).

Cafaro, P. 2009a. Less is more. In Voluntary simpllicity - the poetic alternative to consumer culture, eds. S. Alexander, pp. 127-133. Whanganui: Stead and Daughters Ltd.

Cafaro, P. 2009b. Thoreau's alternative economics. In Voluntary simpllicity - the poetic alternative to consumer culture, eds. S. Alexander, pp. 127-133. Whanganui: Stead and Daughters Ltd.

CBD (Convention on Biological Diversity). 1992. Text of the Convention on Biological Diversity. Http://www.cbd.int/convention/text/ (accessed 5 February 2013).

CBD (Convention on Biological Diversity). 2010a. Global Biodiversity Outlook 3 . Montreal: Secretariat of the Convention on Biological Diversity.

CBD (Convention on Biological Diversity). 2010b. Biodiversity Scenarios: Projections of 21st Century Change in Biodiversity and Associated Ecosystem Services . Montreal: Secretariat of the Convention on Biological Diversity.

CBD (Convention on Biological Diversity). 2011. History of the Convention. Montreal, Canada. <http://www.cbd.int/history/> (Accessed on 23/9/2011).

CBD (Convention on Biological Diversity. 2013. National biodiversity strategies and action plans (NBSAPs). Http://www.cbd.int/nbsap/ (accessed 08 January 2014)

CE Delft, Infras, and Fraunhofer ISI. 2011. External Costs of Transport in Europe. Update Study for 2008 . Delft: CE Delft. 
Chapin III, F.S., E.S. Zavaleta, V.T. Eviner, R.L. Naylor, P.M. Vitousek, H.L. Reynolds, D.U. Hooper, S. Lavorel, O.E. Sala, S.E. Hobbie, M.C. Mack, and S. Díaz. 2000. Consequences of changing biodiversity. Nature 405: 234-242.

Charry, B., and J. Jones. 2009. Traffic volume as a primary road characteristic impacting wildlife: a tool for land use and transportation planning. In Proceedings of the international conference on ecology and transportation, eds. P.J. Wagner, D. Nelson and E. Murray, pp. 159-172. Raleigh: Center for Transportation and the Environment.

Christini, G., D.H. Matthews, and C.T. Hendrickson. 2011. Corporate Environmental Management. Http://gdi.ce.cmu.edu/gd/Research/ems-03.pdf (accessed 28 January 2013).

Chun, Y., and B. Bidanda. 2013. Sustainable manufacturing and the role of the International Journal of Production Research. International Journal of Production Research 51(23-24): 7448-7455.

Cinzano, P., F. Falchi, and C.D. Elvidge. 2001. The First World Atlas of the Artificial Night Sky Brightness. Monthly Notices of the Royal Astronomical Society 328: 689-707.

Club of Rome. 2011. About the Club of Rome. Http://www.clubofrome.org/?p=324 (accessed 23 September 2011).

Corlatti, L., K. Hackländer, and F. Frey-Roos. 2009. Ability of wildlife overpasses to provide connectivity and prevent genetic isolation. Conservation Biology 23: 548-556.

Crompton, T., and T. Kasser. 2009. Meeting environmental challenges: The role of human identity . Surrey: WWF-UK.

Dahm, J.D. 2003. Zukunftsfähige Lebensstile - Städtische Subsistenz für mehr Lebensqualität . Cologne: Cologne University.

Daly H.E. 1977. Steady-state economics: The economics of biophysical equilibrium and moral growth . San Francisco: W. H. Freeman and Company.

Daly, H.E. 1973. Toward a Steady State Economy . San Francisco: W. H. Freeman and Company.

Daly, H.E. 1996. Beyond Growth: The Economics of Sustainable Development . Boston: Beacon Press.

Dawkins, R. 1976. The selfish gene . Oxford: Oxford University Press.

DEGA (Deutsche Gesellschaft für Akustik). 2010. Straßenverkehrslärm - Eine Hilfestellung für Betroffene (Noise from road traffic - An assistance to aprties concerned). ALD - Schriftenreihe 1(2010): 1-76. 
Dekker, R., J. Bloemhof, and I. Mallidis. 2012. Operations Research for green logistics - An overview of aspects, issues, contributions and challenges. European Journal of Operational Research 219: 671-679.

DHL (Deutsche Post DHL). 2013. Taking on responsibility together. Corporate Responsibility Report 2012 . Bonn: Deutsche Post DHL.

DIN (German Institute for Standardization). 2012. Umweltmanagement DIN EN ISO $14001 \mathrm{ff}$. Dokumentensammlung (Environmental Management DIN EN ISO $14001 \mathrm{ff}$. Collection of documents.) . Berlin: Beuth.

Dooling R.J., and A.N. Popper. 2007. The effects of highway noise on birds. Report to the California. Department of Transportation, contract 43AO139 . Sacramento: California Department of Transportation, Division of Environmental Analysis.

Dunlap, J.C. 1999. Molecular bases for circadian clocks. Cell 96: 271-290.

Ecoinvent. 2013. Ecoinvent V3 . Hamburg: Ecoinvent.

EEA (European Environment Agency). 2002. Corine land cover 2000 (CLC2000) seamless vector database. Http://www.eea.europa.eu/data-andmaps/data/corine-land-cover-2000-clc2000-seamless-vector-database (accessed 24 March 2013).

EEA (European Environmental Agency). 2011. Landscape fragmentation in Europe Joint EEA-FOEN report . Copenhagen: EEA.

EEC (European Economic Community). 1985. Council Directive 85/337/EEC of 27 June 1985 on the assessment of the effects of certain public and private projects on the environment (85/337/EWG) Http://eurlex.europa.eu/LexUriServ/LexUriServ.do?uri=OJ:L:1985:175:0040:0048:EN:PD $\mathrm{F}$ (accessed 13 January 2014)

Eichhorn, S. 2013. Naturwissenschaftliche Erkenntnisse und die moralische Bewertung der lebendigen Natur. In Societal dimensions of the conservation and utilization of biological diversity, eds. J. Friedrich, A. Halsband and L. Minkmar, pp. 1733. Goettingen: Goettingen University Press.

Eickermann, A. 2013. Forests - Trapped between the Regimes. In Societal dimensions of the conservation and utilization of biological diversity, eds. J. Friedrich, A. Halsband and L. Minkmar, pp. 275-293. Goettingen: Goettingen University Press.

Eigenbrod, F., St.J. Hecnar, and L. Fahrig. 2009. Quantifying the road-effect zone: threshold effects of a motorway on anuran populations in Ontario, Canada. Ecology and Society 14: 1-18.

EKD (Kirchenamt der Evangelischen Kirche in Deutschland). 2012. Die Erde ist des Herrn und was darinnen ist. Biopatente und Ernährungssicherung aus christlicher Perspektive. Hannover: EKD. 
Elkington, J. 1999. Cannibals with Forks: Triple Bottom Line of 21st Century Business . Bloomington: Capstone Publishing.

Elsner. 2010. Der Elsner. Handbuch für Straßen- und Verkehrswesen . Dieburg: Otto Elsner Verlagsgesellschaft.

EMAS (Eco-Management and Audit Scheme). 2009. Regulation (EC) No 1221/2009 of the European Parliament and of the Council of 25 November 2009 on the voluntary participation by organisations in a Community eco-management and audit scheme (EMAS). Http://eur-

lex.europa.eu/LexUriServ/LexUriServ.do?uri=OJ:L:2009:342:0001:0045:EN:PD F (accessed 11 January 2014)

EMAS (Eco-Management and Audit Scheme). 2010. Die neuen Kernindikatoren der EMAS III. Http://www.emas.de/fileadmin/user_upload/06_service/PDFDateien/UGA_Infoblatt-Indikatoren_Mrz_2010.pdf (accessed 11 January 2014)

EMAS (Eco-Management and Audit Scheme). 2011a. Main Features. Http://ec.europa.eu/environment/emas/about/summary_en.htm (accessed 24 September 2011).

EMAS (Eco-Management and Audit Scheme). 2011b. Legislative Texts. Http://ec.europa.eu/environment/emas/documents/legislative_en.htm (accessed 24 September 2011).

Esswein, H., and H.-G. Schwarz-von Raumer. 2005. LIKI-Indicator "Landscapefragmentation", Identification of undissected low traffic areas and effective mesh size (original title in German: LIKI-Indikator „Landschaftszerschneidung“, Ermittlung der Unzerschnittenen Verkehrsarmen Räume (UZVR) und der effektiven Maschenweite (meff). In Possibilities and limits of undissected low traffic areas for a qualitative assessment, steering, and compensation of land use" (Möglichkeiten und Grenzen der UZVR zur qualitativen Bewertung, Steuerung und Kompensation von Flächeninanspruchnahmen) . Leipzig: Federal Agency for Nature Conservation.

EU (European Union). 2001. Directive 2001/42/EC of the European Parliament and of the Council of 27 June 2001 on the assessment of the effects of certain plans and programmes on the environment. Http://eurlex.europa.eu/LexUriServ/LexUriServ.do?uri=CELEX:32001L0042:EN:NOT (accessed 11 January 2014).

EU (European Union). 2002. Richtlinie 2002/49/EG des Europäischen Parlaments und des Rates vom 25. Juni 2002 über die Bewertung und Bekämpfung von Umgebungslärm. L 189/12 DE. Http://eur-

lex.europa.eu/LexUriServ/LexUriServ.do?uri=OJ\%3AL\%3A2002\%3A189\%3A0 012\%3A0025\%3ADE\%3APDF (accessed 11 January 2014).

Ewing B., A. Reed, A. Galli, J. Kitzes, and M. Wackernagel. 2010. Calculation Methodology for the National Footprint Accounts, 2010 Edition. Oakland: Global Footprint Network. 
Fahrig, L. 2003. Effects of habitat fragmentation on biodiversity. Annual Review of Ecology, Evolution, and Systematics 34: 487-515.

Fahrig, L., and T. Rytwinski. 2009. Effects of roads on animal abundance: an empirical review and synthesis. Ecology and Society 21: 1-20.

FAO (Fisheries and Aquaculture Department). 2009. The state of world fisheries and aquaculture 2008 . Rome: Food and Agriculture Organization of the United Nations.

Federal Statistical Office. 1999. Zur Interpretation und Verknüpfung von Indikatoren (Interlinkages) . Wiesbaden: Federal Statistical Office.

Federal Statistical Office. 2012. Umweltökonomische Gesamtrechnungen. Nachhaltige Entwicklung in Deutschland Indikatoren zu Umwelt und Ökonomie. 2013. Wiesbaden: Federal Statistical Office.

Federal Statistical Office. 2013a. Surface area according to actual use, 2011 (Bodenfläche nach Art der tatsächlichen Nutzung, 2011). Wiesbaden: Federal Statistical Office.

Federal Statistical Office. 2013b. Surface area according to actual use, 2012 (Bodenfläche nach Art der tatsächlichen Nutzung, 2012). Wiesbaden: Federal Statistical Office.

FGSV (German Road and Transportation Research Association). 1996. Guidelines for the design of roads (Richtlinie für die Anlage von Straßen RAS, Teil: Querschnitte RAS-Q) . Cologne: FGSV.

FGSV (German Road and Transportation Research Association). 2011. Guidelines for resting facilities of roads (Empfehlungen für Rastanlagen an Straßen : ERS) . edition 2011. Cologne: FGSV.

Finkbeiner, M. 2009. Carbon footprinting - opportunities and threats. International Journal of Life Cycle Assess 14(2): 91-94.

Fischer, M., and D. Matthies. 1998. RAPD variation in relation to population size and plant fitness in the rare Gentianella germanica (Gentianaceae). American Journal of Botany 85: 811-819.

Fitschen, A., and H. Nordmann. 2012. Verkehrsentwicklung auf Bundesfernstraßen 2010. Bergisch Gladbach: Federal Highway Research Institute.

Forman, R.T.T. 1995. Land Mosaics: The Ecology of Landscapes and Regions . Cambridge: Cambridge University Press.

Forman, R.T.T. 2000. Estimate of the area affected ecologically by the road system in the United States. Conservation Biology 14: 31-35.

Forman, R.T.T., and D. Sperling. 2011. The future of roads: No driving, no emissions, nature reconnected. Solutions 5: 10-23. 
Forman, R.T.T., and R.D. Deblinger. 2000. Ecological Road-Effect Zone of a Massachusetts Suburban Highway. Conservation Biology 14: 36-46.

Forman, R.T.T., B. Reineking, and A.M. Hersperger. 2002. Road Traffic and Nearby Grassland Bird Patterns in a Suburbanizing Landscape. Environmental Management 29(6): 782-800.

Forman, R.T.T., D. Sperling, J.A. Bissonette, A.P. Clevenger, C.D. Cutshall, V.D. Dale, L. Fahrig, R. France, C.R. Goldman, K. Heanue, J.A. Jones, F.J. Swanson, T. Turrentine, and T.C. Winter. 2003. Road Ecology: Science and Solutions . Washington, DC: Island Press.

Foster, J.B. 2013. An interview with John Bellamy Foster. Http://mrzine.monthlyreview.org/2013/foster180713.html (accessed 1 March 2014).

Friedrich, J., and J. Geldermann. 2013a. Potentielle Auswirkungen von Straßenverkehrstransporten auf die Biodiversität. In Treffpunkt Biologische Vielfalt 12: Interdisziplinärer Forschungsaustausch im Rahmen des Übereinkommens über die Biologische Vielfalt, eds. U. Feit and H. Korn, pp. 107-113. Bonn: Federal Agency for Nature Conservation.

Friedrich, J., and J. Geldermann. 2013b. Current practices of mitigating the ecologically harmful effects of roads - An assessment. In Societal dimensions of the conservation and utilization of biological diversity, eds. J. Friedrich, A. Halsband and L. Minkmar, pp. 341-358. Goettingen: Goettingen University Press.

Fromm, E. 1976. To have or to be? . New York: Harpercollins.

Fukuoka, M. 1978. The one straw revolution . Emmaus: Rodale Press.

Gagnon, J.W., T.C. Theimer, N.L. Dodd, S. Boe, and R.E. Schweinsburg. 2007. Traffic volume alters elk distribution and highway crossings in Arizona. The Journal of Wildlife Management 71(7): 2318-2323.

Gallaway, T., R.N. Olsen, and D.M. Mitchell. 2010. The economics of global light pollution. Ecological Economics 69:658-665.

Gerlach, G., and K. Musolf. 2000. Fragmentation of Landscape as a Cause for Genetic Subdivision in Bank Voles. Conservation Biology 14(4):1066-1074.

German Bundestag. 2013. Schlussbericht der Enquete-Kommission „Wachstum, Wohlstand, Lebensqualität -Wege zu nachhaltigem Wirtschaften und gesellschaftlichem Fortschritt in der Sozialen Marktwirtschaft" (Study Commission on Growth, Well-being and Quality of Life-Paths to Sustainable Economic Activity and Social Progress in the Social Market Economy). Drucksache 17/13300, 03.05.2013.

Http://www.bundestag.de/bundestag/gremien/enquete/wachstum/Schlussberich t/17-13300.pdf (accessed 16 January 2014) 
GFN (Global Footprint Network). 2010. National ecological footprint and biocapacity of 2007. Oakland: Global Footprint Network.

GFN (Global Footprint Network). 2013. Earth overshoot day. Http://www.footprintnetwork.org/de/index.php/gfn/page/earth_overshoot_day/ (accessed 03 January 2014)

Gibbs, J.P. and W.G. Shriver. 2002. Estimating the effects of road mortality on turtle populations. Conservation Biology 16(6): 1647-1652.

Gibbs, J.P. and W.G. Shriver. 2005. Can road mortality limit populations of poolbreeding amphibians? Wetlands Ecology and Management 13: 281-289.

Glista, D.J., T.L. DeVault, and J.A. DeWoody. 2008. Vertebrate road mortality predominantly impacts amphibians. Herpetological Conservation and Biology 3(1): 7787.

Goergescu-Roegen, N. 1971. The entropy law and the economic process . Cambridge: Harvard University Press.

Goergescu-Roegen, N. 1973. The entropy law and the economic problem. In Toward a steady-state economy, eds. H.E. Daly, pp. 37-49. San Francisco: Freeman and Company.

Goergescu-Roegen, N. 1979. La décroissance. Entropie - Écologie-Économie . Paris: Édi-tions Sang de la terre.

Google Maps. 2014a. Display of the final part of route 2.

$\mathrm{Https}: / /$ maps.google.com/maps?saddr=A7\&daddr=Sperbersloher+Stra\%C3\%9F e,+Wendelstein, + Deutschland\&hl=de\&ie $=U T F 8 \& \|=49.790131,10.788574 \&$ spn $=$ $1.186233,2.90863 \& s \|=50.396263,10.670471 \&$ sspn $=1.171323,2.90863 \&$ geocod e=FXgZAAMdshWVAA\%3BFVEI8QId1TeqACIneiGHDFufRzEOhNsfLimBow\& $\mathrm{mra}=\mathrm{dme} \& \mathrm{mrsp}=0 \& \mathrm{sz}=9 \& \mathrm{t}=\mathrm{m} \& \mathrm{z}=9$ (accessed 4 March 2014).

Google Maps. 2014b. Route display Hann. Münden to Hannover to Brunswick and back to Hann. Münden.

Https://maps.google.com/maps?saddr=Tonlandstra\%C3\%9Fe,+Hann.+M\%C3 $\%$ BCnden,+Deutschland\&daddr=Ulmer+Stra\%C3\%9Fe,+Laatzen,+Deutschlan $\mathrm{d}+$ to:Bevenroder+Stra\%C3\%9Fe,+Braunschweig,+Deutschland+to:Tonlandstra $\% \mathrm{C} 3 \% 9 \mathrm{Fe},+$ Hann.+M\%C3\%BCnden,+Deutschland\&hl=de\&ie=UTF8\& $=50.190$ $968,12.502441 \& s p n=9.414409,23.269043 \& s \|=51.918862,10.093689 \& s s p n=1$. 087576,2.90863\&geocode=FejkEAMdOGeTACnp_1qGDjC7RzFIUAUGBjNkCw \%3BFRxjHgMdTWiVACIHTUdMXwqwRzHSx6ws1Z5Mog\%3BFT4CHgMdcDah ACkntQSEFPSvRzHY8RRW36hpoA\%3BFejkEAMdOGeTACnp_1qGDjC7RzFI UAUGBjNkCw\&mra=ls\&t=m\&z=6 (accessed 4 March 2014).

Google Maps. 2014c. Route display Hannover to Wendelstein Https://maps.google.com/maps?saddr=Tillystra\%C3\%9Fe,+Hannover,+Deutsch land\&daddr $=$ Sperbersloher + Stra\%C3\%9Fe,+Wendelstein,+ Deutschland\&hl=de \&ie=UTF8\& $\|=50.035974,12.370605 \& s p n=9.444797,23.269043 \& s\|=37.0625$, 95.677068\&sspn=46.27475,93.076172\&geocode=FdKfHgMdsEyUACl1j6ZvJH 
WwRzFrP0tfnSqfpg\%3BFVEI8QId1TeqACIneiGHDFufRzEOhNsfLimBow\&mra $=I s \& t=m \& z=6$ (accessed 4 March 2014).

Gorz, A. 2009. Auswege aus dem Kapitalismus. Beiträge zu einer politischen Ökologie . Zurich: Rotpunktverlag.

Graeber, D. 2011. Debt: The first 5,000 years . New York: Melville House.

Grafe, T.U., S. Döbler, and E.K. Linsenmair. 2002. Frogs flee from the sound of fire. Proceedings of the Royal Society B 269: 999-1003.

Gray, R. 2006. Does sustainability reporting improve corporate behaviour?: Wrong question? Right time? Accounting and Business Research 36: 65-88.

GREENPEACE. 2011. Tanzanian government partially withdraws from Serengeti Highway plan. Http://www.greenpeace-maga-

zin.de/index.php?id=55\&tx_ttnews[tt_news]=114199\&tx_ttnews[backPid] $=54 \& c$ Hash=9c822a2e47820486169317946e0d274d (accessed 24 September 2011).

Gregg, R.B. 2009. The value of voluntary simplicity. In Voluntary simpllicity - the poetic alternative to consumer culture, eds. S. Alexander, pp. 127-133. Whanganui: Stead and Daughters Ltd.

GRI (Global Reporting Initiative). 2006. Sustainability reporting guidelines G3 . Amsterdam: Global Reporting Initiative.

GRI (Global Reporting Initiative). 2011. Sustainability reporting guidelines G3.1 . Amsterdam: Global Reporting Initiative.

GRI (Global Reporting Initiative). 2013. Sustainability reporting guidelines G4 . Amsterdam: Global Reporting Initiative.

Guinée, J.B., M. Gorrée, R. Heijungs, G. Huppes, R. Kleijn, A. de Koning, L. van Oers, A. Wegener Sleeswijk, S. Suh, H.A.U. de Haes, H. de Bruijn, R. van Duin, and M.A.J. Huijbregts. 2001. LCA - An operational guide to the ISO-standards . Leiden: Ministry of Housing, Spatial Planning and the Environment (VROM) and Center of Environmental Science Leiden University.

Halfwerk, W., L.J.M. Holleman, C.M. Lessells, and H. Slabbekoorn. 2011. Negative impact of traffic noise on avian reproductive success. Journal of Applied Ecology 48: 210-219.

Hänel, K., H. Reck, M. Böttcher, M. West, A. Huckauf, J. Jessberger, R. André, M. Herrmann, N. Klar, U. Walz, L. Stratmann, and J. Schumacher. 2010. Prioritäten zur Wiedervernetzung von Lebensraumkorridoren im überregionalen StraBennetz - Netzwerk für Wald bewohnende, große Säugetiere. Http://bfn.de/fileadmin/MDB/documents/themen/eingriffsregelung/2012_Priorita eten_Gro\%DFsaeuger_Layout_A0_300dpi.pdf (accessed 14 March 2013).

Hänel, K., and H. Reck. 2011. National Priorities of reconnecting ecosystems (Bundesweite Prioritäten zur Wiedervernetzung von Ökosystemen: Die Überwindung 
straßenbedingter Barrieren. Ergebnisse des F+E-Vorhabens 350782090 des Bundesamtes für Naturschutz). Naturschutz und Biologische Vielfalt 108: 1-353.

Hardin, G. 1968. The tragedy of the commons. Science 162: 1243-1248.

Harvey, D. 2007. The Limits to Capital . London: Verso.

Haug, F. 2014. Zeit, Wohlstand und Arbeit neu definieren. In Zeitwohlstand - Wie wir anders arbeiten, nachhaltig wirtschaften und besser leben, eds. Konzeptwerk Neue Ökonomie, pp. 26-38. Munich: oekom.

Helldin, J.O., P. Collinder, D. Bengtsson, Å. Karlberg, and J. Askling. 2013. Assessment of traffic noise impact in important bird sites in Sweden - a practical method for the regional scale. Oecologia Australis 17(1): 48-62.

Hels, T., and E. Buchwald. 2010. The effects of road kills on amphibian populations. Biological Conservation 99: 331-340.

Herrmann, M., J. Enssle, M. Süsser, and J.-A. Krüger. 2007. Der NABUBundeswildwegeplan. Meckenheim: Wahrlich-Druck.

Holder, J. 2004. Environmental Assessment: The Regulation of Decision Making . New York: Oxford University Press.

Hölker, F., C. Wolter, E.K. Perkin, and K. Tockner. 2010. Light pollution as a biodiversity threat. Trends in Ecology and Evolution 25: 681-682.

Hopkins, R. 2008. The Transition Handbook: From Oil Dependency to Local Resilience . Foxhole, Dartington, Totnes, Devon: Green Books.

Huijser, M.P., J.W. Duffield, A.P. Clevenger, R.J. Ament, and P.T. McGowen. 2009. Cost-benefit analyses of mitigation measures aimed at reducing collisions with large ungulates in the United States and Canada; a decision support tool. Ecology and Society 14(2): 1-15.

Hursthouse, R. 2013. Virtue Ethics. In The Stanford Encyclopedia of Philosophy (Fall 2013 Edition), eds. E.N. Zalta, pp. 1ff. Stanford: Stanford University.

Http://plato.stanford.edu/archives/fall2013/entries/ethics-virtue/ (accessed 21 December 2013).

Illich, I. 1978. Fortschrittsmythen (Myths of progress) . Reinbeck: Rowohlt.

IPBES (Intergovernmental Platform on Biodiversity and Ecosystem Services). 2011. Frequently asked questions. Http://ipbes.net/about-ipbes/frequently-askedquestions.html (accessed 22 September 2011).

IPCC (Intergovernmental Panel on Climate Change). 2007. Synthesis Report . Valencia: IPCC.

IPCC (Intergovernmental Panel on Climate Change). 2013. Summary for Policymakers. In Climate Change 2013: The Physical Science Basis. Contribution of Working 
Group I to the Fifth Assessment Report of the Intergovernmental Panel on Climate Change, eds. T.F. Stocker D. Qin, G.-K. Plattner, M. Tignor, S.K. Allen, J. Boschung, A. Nauels, Y. Xia, V. Bex, and P.M. Midgley, pp. 1-33. Cambridge: Cambridge University Press.

ISO (International Organization for Standardization). 1996. International standard ISO 14.001. Environmental management systems - Specifications with guidance for use. Genève: ISO.

ISO (International Standardizing Organization). 2011. ISO 26.000 - Social Responsibility.

Http://www.iso.org/iso/iso_catalogue/management_and_leadership_standards/s ocial_responsibility/sr_discovering_iso26000.htm (accessed 24 September 2011).

luell, B., G.J. Bekker, R. Cuperus, J. Dufek, G. Fry, C. Hicks, V. Hlaváč, V.B. Keller, C. Rosell, T. Sangwine, N. Tørsløv, and B.L.M. Wandall. 2003. Wildlife and traffic: a European handbook for identifying conflicts and designing solutions . Utrecht: KNNV Publishers.

Jackson, T. 2009. Prosperity without growth. Economics for a finite planet . London: Earthscan.

Jaeger, J. 2001. Ansätze zur Quantifizierung der Landschaftszerschneidung und die Einbeziehung räumlich-funktionaler Zusammenhänge. In Rolle und Bedeutung von Modellen für den ökologischen Erkenntnis-prozeß. Beiträge zu einer Tagung des Arbeitskreises "Theorie" in der Gesell-schaft für Ökologie vom 1.-3. März 2000 , eds. F. Jopp and G. Weigmann, pp. 115-126. Frankfurt/Main: Peter Lang.

Jaeger, J., S. Grau, and W. Haber. 2005a. Einführung: Landschaftszerschneidung und die Folgen. Gaia 14: 98-100.

Jaeger, J.A.G., J. Bowman, J. Brennan, L. Fahrig, D. Bert, J. Bouchard, N. Charbonneau, K. Frank, B. Gruber, and K. Tluk von Toschanowitz. 2005b. Predicting when animal populations are at risk from roads: an interactive model of road avoidance behavior. Ecological Modeling 185: 329-348.

Jaeger, J.A.G. 2000. Landscape division, splitting index, and effective mesh size: new measures of landscape fragmentation. Landscape Ecology 15: 115-130.

Jaeger, J.A.G., H. Esswein, and H.G. Schwarz von Raumer. 2006. Landschaftszerschneidung in Baden-Württemberg: Fortschreibung zeigt weitere starke Zunahme der Zerschneidung an. Bad Boller Skripte 3 (2006): 17-48.

Jarup, L., W. Babisch, D. Houthuijs, G. Pershagen, K. Katsouyanni, E. Cadum,M.-L. Dudley, P. Savigny, I. Seiffert, W. Swart, O. Breugelmans, G. Bluhm, J. Selander, A. Haralabidis, K. Dimakopoulou, P. Sourtzi, M. Velonakis,and F. VignaTaglianti. 2008. Hypertension and Exposure to Noise near Airports: the HYENA Study. Environmental Health Perspectives 116(3): 329-333. 
Johansson, O. 1996. Welfare, externalities, and taxation; theory and some road transport application. Göteborg: Kompendiet-Göteborg.

Jordaan, S.M., D.W. Keith, and B. Stelfox. 2009. Quantifying land use of oil sands production: a life cycle perspective. Environmental Research Letters 4:1-15.

Kallis, G. 2011. In defence of growth. Ecological Economics 70(2011): 873-880.

Kaseloo, P.A. 2006. Synthesis of noise effects on wildlife populations. In Proceedings of the 2005 international conference on ecology and transportation, eds. C.L. Irwin, P. Garrett and K.P. McDermott, pp. 33-35. Raleigh: Center for Transportation and the Environment.

Keller, I., W. Nentwig, and C.R. Largiadèr. 2004. Recent habitat fragmentation due to roads can lead to significant genetic differentiation in an abundant flightless ground beetle. Molecular Ecology 13: 2983-2994.

Kempf, H. 2008. How the rich are destroying the earth . Foxhole, Darting-ton, Totnes, Devon: Green Books.

Kempf, H. 2010. Democracy, ecological crisis and oligarchy. Plenary presentation in the International Conference on the Climate of Democracy, Parma, 7-8 October 2010. In In defence of growth , G. Kallis. Ecological Economics 70(2011): 873880.

Kerth, G., and M. Melber. 2009. Species-specific barrier effects of a motorway on the habitat use of two threatened forest-living bat species. Biological Conservation 142: $270-279$.

Klink, D. 2008. Der Ehrbare Kaufmann - Das ursprüngliche Leitbild der Betriebswirtschaftslehre und individuelle Grundlage für die CSR-Forschung. Journal of Business Economics 3(2008): 57-79.

Korten, D. 2009. Agenda for a New Economy. From Phantom Wealth to Real Wealth . San Francisco: Berrett-Koehler Publishers, Inc.

Kovel, J. 2002. The Enemy of Nature: The End of Capitalism or the End of the World? . London: Zed Books.

Krebs, A. 1997. Overview of ethics of nature (Naturethik im Überblick). In Naturethik Grundtexte der gegenwärtigen tier- und ökoethischen Diskussion (Ethics of Nature - Fundamental texts of the current animal- and ecoethical discussion), ed. A. Krebs, pp. 334-379. Frankfurt am Main: Suhrkamp.

Krebs, A. 2008. Naturethik (Ethics of Nature).

Http://www.bpb.de/gesellschaft/umwelt/bioethik/33722/naturethik (accessed 20 December 2013)

Krugman, P. 2009. New York Times Magazine, September 6, 2009. In Who Were These Economists Anyway? , J.K. Galbraith. Thought \& Action 25(2009):85-95. 
Kunz, H. 2013. 33111G4001 Tatsächliche Nutzung der Bodenfläche (Gemarkungen) nach 1er-Stellen 31.12.2012 (Land cover by specific use). Bad Ems: Federal Statistical Office Rhineland Palatine.

Lamure, C. 1986. Road traffic noise: Generation, propagation and control. In Noise Pollution, A.L. Sáent and R.W.B. Stephens. Hoboken: John Wiley \& Sons Ltd.

Lassen, D. 1979. Unzerschnittene verkehrsarme Räume in der Bundesrepublik Deutschland. Natur und Landschaft 54: 333-334.

Lassen, D. 1987. Unzerschnittene verkehrsarme Räume über $100 \mathrm{~km}^{2}$ Flächengröße in der Bundesrepublik Deutschland - Fortschreibung 1987. Natur und Landschaft 12: 532-535.

Latouche, S. 2010. Farewell to growth . Cambridge: Polity Press.

Lengagne, T. 2008. Traffic noise affects communication behaviour in a breeding anuran, Hyla arborea. Biiological Conservation 141(2008): 2023-2031.

Lindenmayer, D.B., and J. Fischer. 2006. Habitat Fragmentation and Landscape Change: An Ecological and Conservation Synthesis . Washington, DC: Island Press.

Linz, M. 2004. Weder Mangel noch Übermaß. Über Suffizienz und Suffizienzforschung . Wuppertal: Wuppertal Institut für Klima, Umwelt Energie.

Liu, S.L., B.S. Cui, S.K. Dong, Z.F. Yang, M. Yang, and K. Holt. 2008. Evaluating the influence of road networks on landscape and regional ecological risk - A case study in Lancang River Valley of Southwest China. Ecological Engineering 34(2008): 91-99.

MA (Millennium Ecosystem Assessment). 2005. Ecosystems and Human Well-being: Synthesis . Washington, DC: Island Press.

Mader, H.J. 1979. Die Isolationswirkung von Verkehrsstraßen auf Tierpopulationen, untersucht am Beispiel von Arthropoden und Kleinsaugern der Waldbiozonöse . Bonn-Bad Godesberg: Federal Agency for Nature Conservation.

Mader, H.J. 1984. Animal Habitat Isolation by Roads and Agricultural Fields. Biological Conservation 29: 81-96.

Maibach, M., D. Peter, and B. Seiler. 1999. Ökoinventar Transporte: Grundlagen für den ökologischen Vergleich von Transportsystemen und den Einbezug von Transportsystemen in Ökobilanzen, Technischer Schlussbericht, SPP Umwelt, Schweizer Nationalfonds zur Förderung der wissenschaftlichen Forschung . $2^{\text {nd }}$ edition, Zurich: Infras.

Marsh, D.M., and N.G. Beckman. 2004. Effects of forest roads on the abundance and activity of terrestrial salamanders. Ecological Applications 14(6):1882-1891. 
Marx, K. 1844/ 1978. Economic and philosophical manuscripts of 1844. In The MarxEngels Reader, ed. R.C. Tucker. New York: Norton. or Http://www.mlwerke.de/me/me40/me40_533.htm (accessed 20 December 2013)

Matthews, D., G. Christini, and C. Hendrickson. 2004. Five Elements for Organizational Decision-Making with an Environmental Management System. Environmental Science \& Technology 38: 1927-1932.

McClure, C.J.W., H.E. Ware, J. Carlisle, G. Kaltenecker, and J.R. Barber. 2013. An experimental investigation into the effects of traffic noise on distributions of birds: avoiding the phantom road. Proceedings of the Royal Society $B$ 1773(280): 1-10.

McGinnis, M., and E. Ostrom. 2008. Will lessons from small-scale social dilemmas scale up? In New Issues and paradigms in research on social dilemmas, eds. A. Biel, D. Eek, T. Gärling and M. Gustafson, pp. 189-211. Heidelberg: Springer.

Meadows, D.H., D.L. Meadows, J. Randers, and W.W. Behrens III. 1972. The limits to growth . $2^{\text {nd }}$ edition. New York: Universe Books.

MELS (Ministry of the Environment, Energy and Climate Protection of Lower Saxony). 2014. Environmental map. Http://www.umweltkartenniedersachsen.de/GlobalNetFX_Umweltkarten/ (accessed 23 January 2014)

Memo AG. 2013. Sustainability Report 2013/14 . Greußenheim: Memo AG.

Minkmar, L. 2010. Biopiraterie. Die Privatisierung der Natur durch geistige Eigentumsrechte. Forum Recht 2010(1): 17-20.

MKULV NRW (Ministry for Climate Protection, Environment, Agriculture, Nature Conservation and Consumer Protection of the German State of North RhineWestphalia). 2014. Ambient noise NRW. Http://www.umgebungslaermkartierung.nrw.de/ (accessed 23 January 2014)

Mollison, B. 1979. Permaculture II: Practical design and further theory in permanent agriculture . Stanley: Tagari.

Mollison, B. 2010. Handbuch der Permakultur-Gestaltung . Stainz: PermakulturAkademie im Alpenraum des Österreichischen Instituts für angewandte Ökopädagogik E.R.D.E..

Mora, C., D.P. Tittensor, S. Adl, A.G.B. Simpson, and B. Worm. 2011. How Many Species Are There on Earth and in the Ocean? PLoS Biology 9(8): 1-8.

Moser, B., J.A.G. Jaeger, U. Tappeiner, E. Tasser, and B. Eiselt. 2007. Modification of the effective mesh size for measuring landscape fragmentation to solve the boundary problem. Landscape Ecology 22(2007): 447-459. 
Murphy, P.R., and R.F. Poist. 2000. Green logistics strategies: an analysis of usage patterns. Transportation Journal 40(2): 5-16.

Nussbaum, M.C. 1992. Human Functioning and Social Justice: In Defense of Aristotelian Essentialism. Political Theory 20(2): 202-24.

Nussbaum, M.C. 2000. Women and Human Development. The Capabilities Approach . Cambridge: Cambridge University Press.

Nussbaum, M.C. 2006. Frontiers of Justice. Disability, Nationality, Species Membership . Cambridge/London: Belknap.

ODOT (Ohio Department of Transportation). 2006. Statewide investigation of noise abatement alternatives . Columbus: Ohio Department of Transportation.

Oggier, P., A. Righetti, and L. Bonnard. 2007. Zerschneidung von Lebensräumen durch Verkehrsinfrastrukturen COST $341.2^{\text {nd }}$ edition. Bern: Bundesamt für Umwelt; Bundesamt für Raumentwicklung; Bundesamt für Verkehr \& Bundesamt für Straßen.

Okri, B. 2008. Our false oracles have failed. We need a new vision to live by. October 30, 2008, The Times, London.

Http://www.thetimes.co.uk/tto/law/columnists/article2047920.ece (accessed 14 January 2014)

Olson, M. 1965. The Logic of Collective Action: Public Goods and Theory of Groups . Cambridge: Harvard University Press.

Ostrom, E. 1990. Governing the Commons: The Evolution of Institutions for Collective Action. New York: Cambridge University Press.

Ott, K., and R. Döring. 2006. Grundlinien einer Theorie starker Nachhaltigkeit (Basic arguments for a theory of strong sustainability) In Umwelt-Handeln. Zum Zusammenhang von Naturphilosophie und Umweltethik, eds. K. Köchy and M. Norwig, pp. 89-127. Freiburg, München: Verlag Karl Alber.

Ott, K. 2009a. Guidelines for a Strong Sustainability - A Proposal for Embedding the Three-Pillar Concept. Gaia 18(2009): 25-28.

Ott, K. 2009b. Zur Begründung der Konzeption starker Nachhaltigkeit. In Zwischen Wissenschaft und Politik - 35 Jahre Gutachten des Sachverständigenrates für Umweltfragen, eds. H.-J. Koch and C. Hey, pp. 63-87. Berlin: Sachverständigenrat für Umweltfragen.

Paech, N. 2009. The economy in the aftermath of growth. Einblicke 49: 24-27.

Paech, N. 2012a. Liberation from Excess - The road to a post-growth economy. Munich: oekom. 
Paech, N. 2012b. "Green" growth would be a wonder („Grünes“ Wachstum wäre ein Wunder). Http://www.zeit.de/wirtschaft/2012-06/wachstumskritik-paech (accessed 08 January 2014)

Paech, N. 2014. Suffizienz und Subsistenz: Therapievorschläge zur Überwindung der Wachtumsdiktatur. In Zeitwohlstand - Wie wir anders arbeiten, nachhaltig wirtschaften und besser leben, eds. Konzeptwerk Neue Ökonomie, pp. 40-49. Munich: oekom.

Parris, K.M., and A. Schneider 2008. Impacts of traffic noise and traffic volume on birds of roadside habitats. Ecology and Society 14(1): 29.

Patricelli, G.L., and J.L. Blickley. 2006. Avian communication in urban noise: causes and consequences of vocal adjustment. Auk 123(3): 639-649.

Pimm, S.L. 2004. A Scientist Audits the Earth . New Brunswick: Rutgers University Press.

Pimm, S.L., G.J. Russell, J.L. Gittleman, and T.M. Brooks. 1995. The Future of Biodiversity. Science 269(5222): 347-350.

Poteete, A., and E. Ostrom. 2004. Heterogeneity, group size, and collective action: The role of institutions in forest management. Development and Change 35(3): 43561.

PRé Consultants B.V. 2001. The Eco-indicator 99 - A damage oriented method for Life Cycle Impact Assessment Methodology Report . $3^{\text {rd }}$ edition. Den Haag: Ministerie van Volkshuisvesting and Ruimtelijke Ordening en Milieubeheer.

ProgTrans AG (Prognoses and Strategy Consulting for Transport and Traffic) and IWW (Institut für Wirtschaftspolitik und WIrtschaftsforschung). 2007. Endbericht. Aktualisierung der Wegekostenberechnung. Karlsruhe: ProgTrans AG and Basel: IWW.

Reck, H, K. Hänel, M. Böttcher, J. Tillmann, and A. Winter. 2005. Habitat corridors for humans and nature (Lebensraumkorridore für Mensch und Natur) . Bonn, BadGodesberg: Federal Agency for Nature Conservation.

Reck, H., and G. Kaule. 1993. Straßen und Lebensräume - Ermittlung und Beurteilung straßenbedingter Auswirkungen auf Pflanzen, Tiere und ihre Lebensräume . Bonn: Typo-Druck.

Reck, H., K. Hänel, J. Jessberger, and D. Lorenzen. 2008. UZVR, UFR + Biologische Vielfalt. In Naturschutz und Biologische Vielfalt , ed. Agency for Nature Conservation Germany, pp 1-181. Münster: Landwirtschaftsverlag.

Reck, H., B. Schultz, and C. Dolnik. 2011. Field guide of Holstein Habitat Corridors and the fauna passage Kiebitzholm, Molfsee: Stiftung Naturschutz SchleswigHolstein. 
Reijnen, R., and R. Foppen. 1995. The effects of car traffic on breeding bird populations in woodland. IV. Infuence of population size on the reduction of density close to a highway. Journal of Applied Ecology 32: 481-491.

Reijnen, R., and R. Foppen, C. Ter Braak, and J. Thissen. 1995. The effects of car traffic on breeding bird populations in woodland. Reduction of density in relation to the proximity of main roads. Journal of Applied Ecology 32:187-202.

Reijnen, R., R. Foppen, and H. Meeuvsen. 1996. The effects of traffic on the density of breeding birds in dutch agricultural grasslands. Biological Conservation 75: 255260.

Reijnen, R., R. Foppen, and G. Veenbaas. 1997. Disturbance by traffic of breeding birds: evaluation of the effect and considerations in planning and managing road corridors. Biodiversity and Conservation 6: 567-581.

Reijnen, R., and R. Foppen. 2006. Impact of road traffic on breeding bird populations. In Ecology of transportation. Managing mobility for the environment, eds. J. Davenport and J.L. Davenport, pp. 255-275. Dordrecht: Springer.

Reuter, K. 2013. Sustainability and the good life (Nachhaltigkeit und gutes Leben). In Societal dimensions of the conservation and utilization of biological diversity, eds. J. Friedrich, A. Halsband and L. Minkmar, pp. 11-16. Goettingen: Goettingen University Press.

Reuther, C., and A. Krekemeyer. 2004. On the Way Towards an Otter Habitat Network Europe (OHNE). - Method and Results of an Area Assessment on the European and the German Level. Habitat 15: 1-308.

Rich, C., and T. Longcore. 2006. Ecological Consequences of Artificial Night Lighting, Washington, DC: Island Press.

Ridoutt, B.G., and S. Pfister. 2013. Towards an Integrated Family of Footprint Indicators. Journal of Industrial Ecology 17(3): 337-339.

Riley, S.P.D, J.P. Polinger, R.M. Sauvajot, E.C. York, C. Bromley, T.K. Fuller, and R.K. Wayne. 2006. A southern California freeway is a physical and spatial barrier to gene flow in carnivores. Molecular Ecology 15: 1733-1741.

Roedenbeck, I.A., and J.A.G. Jaeger. 2006. Auf dem Weg zu straßenökologischer Forschung auf Landschaftsebene. Naturschutz und Landschaftsplanung 38: 297299.

Eversheim, R., H. Reck, and K. Pleuser. 2009. Wald, Wild und biologische Vielfalt. Aufbruch in einen neuen gesellschaftlichen Dialog . Bonn: Stiftung natur+mensch.

Rolston III, H. 1994. Value in Nature and the Nature of Value. In Philosophy and the Natural Environment, Royal Institute of Philosophy Supplement 36 , eds. R. Attfield and A. Belsey, pp. 13-31. Cambridge: Cambridge University Press. 
Rosa, H. 2014. Resonanz statt Entfremdung, Zehn Thesen wider der Steigerungslogik der Moderne. In Zeitwohlstand - Wie wir anders arbeiten, nachhaltig wirtschaften und besser leben, eds. Konzeptwerk Neue Ökonomie, pp. 62-72. Munich: oekom.

Sala, E.O., F.S. Chapin III, J.J. Armesto, R. Berlow, J. Bloomfield, R. Dirzo, E. HuberSanwald, L.F. Huenneke, R.B. Jackson, A. Kinzig, R. Leemans, D. Lodge, H.A. Mooney, M. Oesterheld, N.L. Poff, M.T. Sykes, B.H. Walker, M. Walker, and D.H. Wall. 2000. Global Biodiversity Scenarios for the Year 2100. Science 287: 1770-1774.

Samuelson, P. A. 1954. The Pure Theory of Public Expenditure. The Review of Economics and Statistics 36: 387-389.

Schneider, F., G. Kallis, and J. Martinez-Alier. 2010. Crisis or opportunity? Economic degrowth for social equity and ecological sustainability. Journal of Cleaner Production 18(2010): 511-518.

Schupp, D. 2005. Umweltindikator Landschaftszerschneidung: Ein zentrales Element zur Verknüpfung von Wissenschaft und Politik. Gaia 14: 101-106.

Seidl, I., and A. Zahrnt. 2010. Postwachstumsgesellschaft. Konzepte für die Zukunft . Marburg: Metropolis-Verlag.

Seiler, A. 2005. Predicting locations of moose-vehicle collisions in Sweden. Journal of Applied Ecology 42: 371-382.

Selva, N., S. Kreft, V. Kati, M. Schluck, B.G. Jonsson, B. Mihok, H. Okarma, and P.L. Ibisch. 2011. Roadless and Low-Traffic Areas as Conservation Targets in Europe. Environmental Management 48: 865-877.

Semlitsch, R.D., T.J. Ryan, K. Hamed, M. Chatfield, B. Drehman, N. Pekarek, M. Spath, and A. Watland. 2007. Salamander Abundance along Road Edges and within Abandoned Logging Roads in Appalachian Forests. Conservation Biology 21: 159-167.

Sen, A. 1999. Development as Freedom . New York: Knopf.

Shanley, C.S., and S. Pyare. 2011. Evaluating the road-effect zone on wildlife distribution in a rural landscape. Ecological Society of America 2: 1-16.

Slabbekoorn, H., and E.A. Ripmeester. 2008. Birdsong and anthropogenic noise: implications and applications for conservation. Molecular Ecology 17(1): 72-83.

Smith, A. 1776. The Wealth of Nations . London: W. Strahan and T. Cadell.

Smith, T.M., and R.L. Smith. 2009. Ökologie - Vom Organismus bis zum Ökosystem . 6th edition. Hallbergmoos: Pearson. 
Spielman, D., B.W. Brook, and R. Frankham. 2004. Most species are not driven to extinction before genetics factors impact them. Proceedings of the National Academy of Sciences, USA 101: 15261-15264.

Spielmann, M., Ch. Bauer, R. Dones, and M. Tuchschmid. 2007. Transport Services. ecoinvent report No. 14 . Dübendorf: Swiss Centre for Life Cycle Inventories.

Stengel, O. 2011. Suffizienz - Die Konsumgesellschaft in der ökologischen Krise. Munich: oekom.

Stoner, D. 1925 . The toll of the automobile. Science 61: 56-57.

Strein, M., U. Müller, and R. Suchant. 2005. Artunspezifische Modellierung einer Korridor-Potenzial-Karte für Mitteleuropa - Methodik und erste Ergebnisse einer landschaftsökologischen GIS-Analyse. In Naturschutz und Biologische Vielfalt , ed. Agency for Nature Conservation Germany (BfN). Bonn: BfN.

Taylor, B.D., and R.L. Goldingay. 2010. Roads and wildlife: impacts, mitigation and implications for wildlife management in Australia. Wildlife Research 37: 320331.

Taylor, P.D., L. Fahrig, K. Henein, and M. Gray. 1993. Connectivity Is a Vital Element of Landscape Structure. Oikos 3(68): 571-573.

Tilman, D., R.M. May, C.L. Lehman, and M.A. Nowak. 1994. Habitat destruction and the extinction debt. Nature 371: 65-66.

Transition Network. 2013. About Transition Network.

Http://www.transitionnetwork.org/about (accessed 22 December 2013)

Tullock, G. 2005. Public goods, redistribution and rent seeking . Cheltenham, Massachusetts: Edward Elgar Publishing Limited.

Turner, J.M. 2006. Conservation science and forest service policy for roadless areas. Conservation Biology 20: 713-722.

UBA (Federal Environmental Agency), DLR (German Aerospace Center), DFD (German Remote Sensing Data Center). Corine Land Cover - Germany. To be ordered free of charge at

Http://www.corine.dfd.dlr.de/mod/ext/corine/order_form_en.html.

UBA (German Federal Environmental Agency) \& BMU (Federal Ministry for the Environment, Nature Conservation and Nuclear Safety). 2010. Umweltbewusstsein in Deutschland 2010 - Ergebnisse einer repräsentativen Bevölkerungsumfrage . Niestetal: Silber Druck.

UBA (German Federal Environmental Agency) \& BMU (Federal Ministry for the Environment, Nature Conservation and Nuclear Safety). 2012. Umweltbewusstsein in Deutschland 2012 - Ergebnisse einer repräsentativen Bevölkerungsumfrage . Paderborn: Bonifatius $\mathrm{GmbH}$. 
UBA (German Federal Environmental Agency). 2006. Richtlinie über die Bewertung und Bekämpfung von Umgebungslärm - Auslösekriterien für die Lärmaktionsplanung. Dessau-Roßlau: UBA.

UBA (German Federal Environmental Agency). 2012. Daten zum Verkehr. Ausgabe 2012. Berlin: KOMAG mbH.

UBA (German Federal Environmental Agency). 2013. Treibhausgasausstoss in Deutschland 2012 -vorläufige Zahlen aufgrund erster Berechnungen und Schätzungen des Umweltbundesamtes. Dessau-Roßlau: UBA.

UN (United Nations) and UNFCCC (United Nations Framework Convention on Climate Change). 2010. Report of the Convention of the Parties on its fifteenth session held in Copenhagen, from 7 to 19 December 2009 - Copenhagen Accord. Http://unfccc.int/resource/docs/2009/cop15/eng/11a01. pdf\#page=4 (accessed 22 September 2011).

UN (United Nations). 1992. Agenda 21. New York: United Nations.

UN (United Nations). 2001. United Nations - International Decades Designated by the General Assembly. Http://www.nalis.gov.tt/National-UN-

Days/UN_INTERNATIONALDECADES.html (accessed 22 September 2011).

UNA-Canada (United Nations Association in Canada). 2011. International Decades. Http://www.unac.org/en/news_events/un_days/international_decades.asp (accessed 22 September 2011).

UNEP (United Nations Environmental Program). 2010. Annual Report 2009 . Nairobi: Publishing Services Section.

Uzawa, H. 1999. Toward a general theory of social overhead capital. In Markets, Information and Uncertainty: Essays in Eco-nomic Theory in Honor of Kenneth J. Arrow, eds. K.J. Arrow and G. Chichilnisky, pp. 253-304. Cambridge: Cambridge University Press.

Van der Grift, E.A., R. Van der Ree, L. Fahrig, S. Findlay, J. Houlahan, J.A.G. Jaeger, N. Klar, L.F. Madriñan, and L. Olson. 2013. Evaluating the effectiveness of road mitigation measures. Biodiversity and Conservation 22: 425-448.

Van der Ree, R., J.A.G. Jaeger, E.A. Van der Grift, and A.P. Clevenger. 2011. Effects of roads and traffic on wildlife populations and landscape function: road ecology is moving towards larger scales. Ecology and Society 1(16): 48.

van Griethuysen, P. 2010. Why are we growth-addicted? The hard way towards degrowth in the involuntary western development path. Journal of Cleaner Production 18(6): 590-595.

Varian, H. R. 1992. Microeconomic analysis . $3^{\text {rd }}$ edition, New York: Norton.

Vaude. 2013. Sustainability Report 2013 . Tettnang: Vaude. 
VBUS. 2006. Bekanntmachung der Vorläufigen Berechnungsverfahren für den Umgebungslärm nach $\S 5$ Abs. 1 der Verordnung über die Lärmkartierung (34. BlmSchV) vom 22. Mai 2006. Bundesministerium der Justiz. Bundesanzeiger 58 (154a).

Https://www.kiel.de/leben/umwelt/laerm/_dokumente/bundesanzeiger_154a.pdf (accessed 11 March 2014).

von Hauff, M., and A. Kleine. 2009. Sustainability in 3D - A Plea for Three Dimensions of Sustainability. Gaia 18/1(2009): 29-31.

Weidema, B.P., M. Thrane, P. Christensen, J. Schmidt, and S. Lokke. 2008. Carbon Footprint - A Catalyst for Life Cycle Assessment? Journal of Industrial Ecology 12(1): 3-6.

Werner, G.W. 2008. Einkommen für alle (Basic income for all). Cologne: Bastei Lübbe $\mathrm{GmbH} \&$ Co. KG.

WHO (World Health Organization). 2009. Night Noise Guidelines . Copenhagen: WHO.

Wilkinson, R., and K. Pickett. 2009. The Spirit Level: Why More Equal Societies Almost Always Do Better . London: Allen Lane.

Wilson, E.O. 1984. Biophilia . Cambridge: Harvard University Press.

Wittgenstein, L. 2001. Philosophische Untersuchungen. In Philosophische Untersuchungen. Kritisch-genetische Edition, ed. J. Schulte, pp. 1-1164. Frankfurt: Wissenschaftliche Buchgesellschaft.

Wolters, G. 1995. "Rio" oder die moralische Verpflichtung zum Erhalt der natürlichen Vielfalt - Zur Kritik einer UN-Ethik. Gaia 4(1995): 244-249.

Wood, W.E., and S.M. Yezerinac. 2006. Song Sparrow (Melospiza melodia) song varies with urban noise. Auk 123: 650-659.

WCED (World Commission on Environment and Development). 1987. Our Common Future. Oxford: Oxford University Press.

Wu, H., and S. Dunn. 1995. Environmentally responsible logistics systems. International Journal of Physical Distribution \& Logistics Management 25(2): 20-38.

Yahner, R.H. 1998. Changes in Wildlife Communities near Edges. Conservation Biology 2(4): 333-339.

Zhang, Y., A. Baral, and B.R. Bakshi. 2010. Accounting for Ecosystem Services in Life Cycle Assessment, Part II: Toward an Ecologically Based LCA. Environmental Science \& Technology 44(7): 2624-2631. 


\section{Appendix}

\section{A Discussion of the Meaning of Sustainability and of the Impli- cations}

To define the meaning of sustainability is not a straightforward procedure. While it is the central concept being used when discussing humanly evoked impacts on the environment, since the presentation of the Brundtland-Report in 1987 and the adoption of the Agenda 21 in 1992, which is the ensuing international program of developmental and environmental politics, sustainability is viewed in a broader context of development (WCED 1987, UN 1992). Its tie to development adds a cultural and economic dimension and an emphasis on the countries of the Southern hemisphere. In Western countries the term evolved no longer to be used uniquely for impacts on and responses of the environment. Rather a three-dimensional character of sustainability, where its scope is subdivided to ecological, economic, and social concerns, as for instance in the Triple Bottom Line concept of Elkington (1999), became the common comprehension of the concept.

In the past, there has been extensive discussion about the appropriateness of extending the scope of sustainability to non-ecological areas. Therefore, a universally valid definition, or even one on the meaning of environmental sustainability alone, does not exist. Considering all three pillars as parts of sustainability has made it easier for organizations to use it as a selling concept in different contexts. However, incomprehensive approaches and sporadic actions cannot legitimate the usage of the term to represent an entity's total relation toward its natural environment. There is a clear difference between 'more sustainable' and 'sustainable' behavior, with the latter probably being exercised only by very few communities around the world. While discourse and development of a term are important for its assimilation within society, it seems unlikely that existing, varying interpretations will allow finding a standardized definition in the near future. Following a saying of the philosopher Ludwig Wittgenstein, who said that "the meaning of a word is its use in communication" (Wittgenstein 2001, p. 425), the threedimensional meaning of sustainability would have to be viewed as the current state of the art.

Irrespective whether sustainability at this point should consider ecological issues only, or whether the term can be used also to describe social and economic permanency (see von Hauff \& Kleine 2009), or whether not associating sustainability with neither the one-column nor with the three-pillar model, but rather with the components of efficiency, consistency and sufficiency, or whether an again other approach is preferred, the 
current, vague application of the term seems to be misleading more than to be helping. Sustainability is a term of positive connotation, but in nowadays it is used for marginal improvements that support any form of perpetuity, which do not have to yet mean that a given ecological state can be maintained. Concerning this issue, Ott criticizes the three-pillar model as the "ultimate softener of the idea of sustainability" (Ott 2009a, p. 26).

While the primary goal of this work is to suggest measures that allow for a better understanding and more comprehensive corporate management of the ecological effects of transport, some further analysis of the term sustainability is viewed indispensable in order to clarify the meaning of sustainability assumed in this work as well as to motivate entities for a precise communication of their definition of sustainability. In this work, a one column approach is chosen. The main reason leading to this understanding resides in the development of the German term for sustainability, which is 'Nachhaltigkeit'. The first utilization of the idea dates back to 1713, originating from and since then being employed in the field of forestry (Ott 2009b). Since the development of furnaces in the 14th century, immense amounts of firewood were used for steel production. 'Nachhaltigkeit' was an approach to allow for continually high timber harvesting, advising over a course of time to chop down only as much wood as would naturally grow back during that time. The principle at that time was clearly tied to human use of natural resources. It further proclaims the idea of 'strong sustainability', meaning that natural resources cannot be substituted by any other form of gain or progress, for instance in technology, but that they should be maintained as expressed for instance by the 'constant nature capital rule (CNCR)' of Ott and Döring (2006). Inter alia, their concept builds on the reasoning of Daly, who disagreed with the infinitely substitutability of capital stocks to be appropriate to include natural capital, primarily for the negantropic character of complex biospheric processes, which he considers the evolutionary dowry of humankind that we should respect and preserve (Daly 1996). Exercising the understanding of strong ecological sustainability in practice, the concepts of efficiency, consistency, and sufficiency can be applied, with sufficiency clearly being the silver bullet (Linz 2004, Paech 2012, German Bundestag 2013). Ott (2009a) suggests the comprehensive resilience of threatened natural organisms and systems to be adopted as the most important guideline for sustainability.

Regarding the further development of the meaning of the term, which results in today's common understanding of sustainability, the appearance of the term 'sustainable development' in the Brundtland-Report in 1987 was significant. The central message of the report constitutes that "sustainable development is development that meets the needs of the present without compromising the ability of future generations to meet their own needs" (WCED 1987, p. 1). The broad scope of the statement made the 
meaning of sustainable development and the concept of sustainability rather vague. This condition was utilized in the future and resulted in the formation of the three-pillar model, which was accepted by decision-makers because it granted them comfortably much leeway on how to act in keeping with the principle (Ott 2009b). One can argue that the enlarged scope and the tie to non-industrialized countries' development as of Western vision did not facilitate for people to ponder their lives' balance to and responsibility for a concept that was difficult to apply. Neither did it animate them to contemplate their relation to the subjects of sustainability, which are past, present, and future life forms, including the human being. As indicated in section 2.2, it seems that, ethically, humans would do good at least to reclaim an estimation of respect towards nature. Reuter convincingly argues that "sustainability is an aspect of the good life, because we cannot separate the answer to the question in what kind of world we want to live, namely in a world without environmental crisis, from the answer to the question of how we want to live, namely using the room of maneuver of the individual to support ecological sustainability" (Reuter 2013, p. 5). While the discussion on sustainability in literature extends further, the detail of explanation given in this text and reasoning to assume a one pillar understanding in this work end here. 


\section{B Five Economic Constraints to Sustainable Action - A More Detailed Analysis}

In this part of the appendix, the five structural constraints to sustainable action at a corporate and economic level designated in section 2.3.2 are analyzed to greater detail. At first, the two related inefficiencies of market economies, external effects and the prisoners' dilemma, are referred to. Next, and despite having been addressed more extensively in the body of this work, the suitability of structural GDP, capital, and consumptive growth, again is questioned and additional aspects of the potential alternative strategy of degrowth are described. Last, and to a not as elaborate extent, the subjects of consumer sovereignty and capital sovereignty are analyzed.

\section{On External Effects}

While the main reason for external effects to be referred to here is their relevance with regard to using natural resources and affecting non-human life forms and ecosystems in an inefficient and undesirable way, external effects are directly related to the main theme of this work, which is to quantify potential ecological impacts of road transport, because recognition and accountability are two important prerequisites for a potential just and effective internalization of insufficiently well managed environmental effects.

Also referred to as 'external costs', the phenomenon describes the negative impacts on a third party, which can be a person, a building, a frog, or a mountain, that the originators responsible for the impacts do not or do not fully compensate for (Johansson 1996). The term 'external cost' is put in inverted commas because one may have doubts about the suitability to describe negative effects as costs. This is because the word cost suggests that all effects can be monetized, which is problematic because it is one principal character of external effects that many impacts for instance on health or non-human life forms cannot be expressed well by a pecuniary value or that this cannot be done unambiguously. Although objectifying effects in order to account for them by some means is an improvement compared to not considering them at all, monetizing them in some instances may increasingly disconnect parties, making it more probable for an effect to reoccur, and thus may serve to quieten one's conscience and to find a way to live with them instead of to avoid them. One possibility to facilitate avoidance, is to find maximum limits for instance for countries or individuals, which can either guide or severely restrict subjects. In order to apply a mechanism of 'caps' society would have to discuss thoroughly, which activities shall be selected, because people would be affected to differing degrees. As external effects often concern complex, individually diversely presumed and abstract relational issues like health, property, beauty, or human relation to other life forms or future generations, which was found to be of im- 
portance for a significant number of people to sense joy and fulfilment in life, it seems adequate to set ambitious limits, attempting not to violate the moral values of minorities or even individuals. Further discussion on how to deal with external effects in the future is needed. The term 'external cost' from here on is substituted by the term external effect. The term external effect refers to negative effects only. For a consequence to be classified an external effect, the assessment of an individual is sufficient and no majority is needed, although, ideally, chances that harmful effects are avoided should rise as the number of subjects vocalizing their dismay increases. It can be said that the magnitude of external effects present in a society says something about the quality of a community. There exist four potential, principal reasons, why external effects exist:

1. External effects are sometimes difficult to detect and even more difficult to quantify because they often concern abstract and highly individual values. If a new bypass road that shall be constructed intersects and degrades an ecologically valuable area that is also used by humans for recreational activities, how are the two affected parties to be reimbursed? Do they have a right to be reimbursed at all and can compensation be achieved monetarily? For vermin, anurans, or native plants, this is not possible. Also that the reach of substance emissions or noise is unclear makes, a just quantification very difficult. The example continues to be complicated, when trying to identify the originator of the impact. Is it the elected politicians, who decided in favor of the construction making the tax-payer the entity to render compensation, or should the users of the road raise the amount to be reimbursed in money or in form of an area? The example illustrates the complexity of determining and dealing with external effects and it reveals that much societal debate on the valuation of more abstract values is necessary, if external effects are to be dealt with by the means of compensation. Further, it is to be borne in mind that abstract external effects often are perceived by minorities, which challenges the concept of majority rule in governing (cp. Tullock 2005).

2. The second reason deals with the nature of the human being. There is no clear consent on whether humans are more selfish or altruistic, and while they certainly hold both sentiments, maybe such a dualistic differentiation of motivation is actually inadequate. Disregarding all other reasons, external effects could be hypothesized to exist because the human being may be predominantly selfish, deliberately accepting impacts harmful to others. However, recognizing the various additional factors that can lead to the occurrence of external effects and the indications that oppose to a general dominance of selfishness in the human character, such as studies that prove that levels of joy and fulfillment are higher in people when they can contribute to a community goal than when they enjoy something alone, not sharing it with others, leave the proposition of egoism to be a substantial reason for external effects to occur to be heavily contestable. 
3. The third reason deals with the surroundings in which external effects are more likely to occur. Community structure and group size are thought to have an influence on the type of dominant behavior in humans. One hypothesis is that the more complex structures of life are, the less incentivized are actions that consider the well-being of others. Complexity is created by the number of people one interacts with and by distance or missing contact (Olson 1965). Both features make the relationship between people less personal and more instrumental as impacts of personal decisions on others are not seen. An example of dissimilar degrees of relatedness and complexity would be buying grapes from a local farmer or as a product of Israel in a supermarket. In the latter case it is impossible to check if people and the soil were treated according to one's values.

Two qualities of smaller and more simply structured communities that favor behavior not to affect others negatively are the reciprocity relation and the power to punish (McGinnis \& Ostrom 2008). Repeated contacts nourish the reciprocity thought of not doing to others what one does not want to be done to oneself because a betrayed person would soon be met again. Secondly, in a reliable group setting the control exerted by others is more powerful than that of an individual (Ostrom 1990). These two conditions function as guard rails within which altruistic behavior works better than egocentric behavior. While a certain group size is necessary to apply the punishment mechanism, the assumed negative relation between group size and collective action, which supposes free-riding to be more prevalent in larger groups, over time was relativized, inter alia by Poteete and Ostrom (2004), who stress the significance of the context as well as the role of institutions to soothe the harmful effects of large group size. While it seems that group size must not necessarily correlate negatively with collective action, also Smith (1776) though that his axiom that intentions and behaviors best for one's own security would render the greatest value to an entire community would work best in smaller groups and contexts as he opposed to large transnational corporations, which he found unresponsive to local affairs and unable to exert good stewardship of resources. In his mind ideal economics were characterized by small local economies that interacted with each other and that were guided by the enlightened self-interest of individuals (Smith 1776).

4. The fourth and final reason of external effects referred to here is property rights on land and its natural resources. Hand in hand with the notion of property goes the mindset of considering land as a commodity rather than as community when one person would be a steward maybe because she or he is particularly good at keeping the rights and interests of all in mind. Effects can occur in the originating area, as for instance in the cases of land use change or overexploitation, and in adjacent area or also globally as substances harmful to buildings, human health, or ecosystems and their life forms disseminate by water and air. External effects can concern contemporaries, who can 
be hurt in their values, their feeling of security, or in their relation to all life forms, future generations, and non-human life forms, assuming they are granted some innate value. 7

On a national basis, ideally rules exist that aim at allowing people the most individual freedom possible without compromising the freedom and well-being of others. Despite the foundation of the United Nations, which focuses on worldwide peace and human rights, on a global basis, the subject of private or national ownership or responsibility over natural resources has advanced insufficiently in previous decades. One of the first organizations to have dealt with the issue is the CBD (Convention of Biological Diversity, founded 1992). Although some success has been achieved for instance in the first Kyoto Protocol on limiting greenhouse gas emissions or lastly in the Nagoya Protocol on access and benefit sharing of natural resources in 2010, binding, ambitious, and effective international laws on nature conservation or pollution remain scarce. This is because it is a difficult cultural, philosophical, and political challenge, if for instance people in Germany should have anything to say about the clearing of rainforest or if Brazilians should have a veto on fracking in Germany. 8 While mankind historically has

7 Property rights on organic matters are an ongoing discussion in patenting, where significant consequences can come into effect (see EKD 2012). Supporters argue that the inventor of a new agricultural breed should have exclusive right over the invention, just like individuals or entities have in other industries, because without being granted the right large investments could no longer be recovered and hence products that could be improved would no longer or at least much slower and less intensively be worked on. Critics oppose that natural life forms are fundamental to human life and that it hence is not possible to exclude people from their use or, particularly with regard to property, to infringe one's responsibility of stewardship over areas and forms of life. A second argument is that forms of life do not belong to mankind in a material way and hence neither can belong to people or a group of people in particular (see Minkmar 2010 for further discussion).

8 The discussion if there are, and if yes, what common environmental resources there are is only at its beginning. While most nations agree that all countries are responsible for emitting 'just' amounts of greenhouse gases per capita (and already in determining the quantitative meaning of 'just', due to differing emission histories, states of industrialization, geologic conditions and differing possibilities of consumer- or producer-orientated accounting methods is a great political task), the global rights and responsibilities regarding life and its diversity in the future are likely to become a more and more significant topic of international debate. For instance do some people think that while the territory of the Brazilian rainforest could be under Brazilian leadership, the Amazonian rainforest itself were a natural phenomenon of such richness and importance, that Brazil had a responsibility to maintain it. Brazil in turn does not want its land use policy and the respective economic potential influenced by non-Brazilians. The most prominent proposition along these lines is the REDD mechanism, which would create a financial incentive to 'Reducing Emissions from Deforestation and forest Degradation'. However, it is argued that emission trading with existing forests is dubious because various problems with regard to accounting, uncertain property rights and the more and more acknowledged ecological-social multifunctionality of forests occur (cp. Eikermann 2013). Morally, this approach would lead to further commercialize nature, with problematic social impacts particularly for indigenous peoples and traditional forest users who would have to provide their basis of living as a tradable service (Fatheuer 2010). 
not had much time to ponder on how to use and treat ecosystems globally, urgency for advances and humans' interests may motivate to assign limits, rights, and responsibilities that not only safeguard the existence of the planet, but that aid in minimizing external effects and in augmenting the solidarity and quality of life of all. Thus, there is potential for true development in a true challenge. However, regarding current social and economic structure, one hypothesis is that the more spheres of life, such as nutrition, occupation, or education, are organized via impersonal, competitive markets, the higher gets the probability that people become less sensitive to take note of and to challenge external effects, one of which is humans' enjoyable persistence on the earth.

\section{The Prisoners' Dilemma}

While external effects are usually known, but there is no consensus on their acceptability or there exists no concept on how to internalize or do away with them that is agreed upon or working successfully, in a situation of incentives that is described as the Prisoners' Dilemma, an ideal outcome is known and agreed upon, and vet not attained. While the motivational conditions of an entity that are assumed under the Prisoners' Dilemma are simplified, it seems that the model applies to businesses' actions and governments' policies at both a regional and global level. The dilemma is portrayed by game theory and can be applied to the use of public goods. It displays a situation of motivational conditions, when dominant actions lead to inefficient results. As the scenario of the Prisoners' Dilemma only is one in a number of theories, following its subsequent discussion, findings of alternative approaches are referred to as well.

Public goods in economics are characterized as being nonrival, which means that the consumption of a good by person $A$ does not reduce its availability to $B$, and nonexcludable, which means that A's consumption of the good does not exclude $B$ from consuming it, too (Samuelson 1954, Varian 1992). Examples would be taking a walk in London's Hyde Park, taking a breath, or watching TV. While in reality the two characteristics do not always apply to one hundred per cent, for a group of goods non-rivalry and non-excludability in consumption are valid to such a high degree that individual and organizational behavior that concerns their use can be hypothesized by the two properties. In the case of environmentally sustainable behavior that, inter alia, implies a meaningful preservation of biodiversity, an inefficient total result derives from individual entities' activities: prominently, the Stern Review, also entitled 'The Economics of Climate Change', and the TEEB study on 'The Economics of Ecosystems and Biodiversity' conclude that the economic value of a consistent supply of nature's resources provided by intact ecosystems outweighs the benefits obtained from exploiting and degrading the like systems at a particular point in time (Stern 2007, Süß 2011). Concerning climate change, Stern estimated that investments of $1 \%$ of global GDP per year 
would be sufficient to prevent future climate change damage, which he expected on average to cost between 5 and 20\% of global yearly GDP (Stern 2007). Therefore, assuming decision criteria of maximal resource use, for the global economy as a whole it would be efficient to draw upon natural resources and to degrade ecosystems only to a point that guarantees sufficient provision of the demanded goods and services in the future. As it is hard to assess, which amount of which environmental good will be needed in the future, and as the ongoing decrease of the majority of ecosystems is not expected to go on gradually because tipping points of ecosystem functionality are assumed to exist, application of the precautionary principle can help not to presume the level of tolerable ecosystem degradation too optimistically (CBD 2010a, Chapin III et al. 2000). Studies show, that for a few ecosystems like coral and costal systems and marine life, thresholds of sustainable usage have been surpassed (MA 2005, FAO 2009).

With regard to the actions and incentives of businesses in the whole world, and similarly also of individuals and governments, actions or investments that would yield an efficient result are foregone because in spite of having a common goal, parties do not act as a collective mind. The world economy does not act as one single player, but consists of rivaling national economies and rivaling businesses within the global economy. The world economy hence consists of millions of independent businesses that depend on selling their goods and services. Apart from the influence from regulation, most of these individual entities would have to choose to do business more sustainably. Yet, it can be assumed that their motivational situation consists of dominant actions that lead to an overall inefficient result. In the following, this condition is illustrated by an example from agriculture. If a single poultry farmer abstains from feeding genetically modified organisms (GMOs) or uses only local fodder, the farmer would incur higher costs (the farmer will be referred to as player A). Assuming that other farmers decided to stick to the old sourcing practices, the payoff for player $A$ is negative because player $A$ incurs the entire cost while the small environmental benefit divides between all players (see negative value of player $A$ in field I-ii in Table Ap.1; the first digit of each pair refers to player $\mathrm{A}$, the later to player $\mathrm{B}$ ). It is assumed that the environmental benefit takes the form of a pecuniary value. The fraction of the entire payoff reaching player $A$ does not surpass the incurred cost, unless player $B$ (here representing the majority of other farmers and all other players of the economy that provoke a similar environmental impact or profit from the gained environmental benefit) also invested into a more environmentally friendly production, which would result in large environmental benefit to be divided between all (see field I-i). Hence, the individual farmer, player A, depends on the collaboration of others in order to yield a positive personal payoff for her or his environmental investment. 
As some of the other players are rivals of A player A may feel too much uncertainty about a significant share of other players also to invest and she or he may fear rivaling players' competition. This can result in the situation described by the Prisoners' Dilemma. It usually assumes that players cannot communicate with each other or influence each other, a condition that can be viewed to represent the complex structure of surroundings of player $A$. The condition of incentives that is called a dilemma refers to the situation where the payoffs for both players can be of the kind that regardless of the decision of one of the two players for the other it always yields a payoff not to invest. A situation like this, when a particular strategy always earns a player a larger payoff than another, regardless of what other players do, in game theory is called strategic dominance. The values of the players' payoffs in Table Ap.1 visualize this condition. For player A it can be seen that regardless of player B's decision either to invest or not to do so, player $A$ always attains a higher profit when not investing because 8 is larger than 5 and -1 larger than -2 . This applies to player $B$ vice versa. When players can make a decision only once and when they want to yield the highest payoff possible, a distribution of payoffs as in the Prisoners' Dilemma always leads to a results, in which no player invests. The outcome is surprising insofar as it is inefficient because there exists another pair of actions that sets both players better off (compare field II-ii to field $\mathrm{I}-\mathrm{i})$. This dilemma of inefficient results in game theory became popular as the 'Prisoners Dilemma' (Straffin 1980). In the classical example, the distribution of payoffs makes two culprits confess and go to jail instead of having left the hearing as free men had they both not confessed. With regard to the probability of the situation depicted above correctly to represent competition in global markets, it must be criticized that the assumptions that there is no communication and no phases are unrealistic. However, while this would have been the conditions as of the original Prisoners' Dilemma, here it can be assumed that players of an entire economy can interact and carry out actions repeatedly over a course of time. Hence, the underlying assumption in the farmers' example would have been that the pictured state of payoffs was one that became constant over time.

Table Ap.1: Situation of payoffs that provokes an inefficient result (Source: own representation).

\begin{tabular}{|c|c|c|c|c|}
\hline & $\mathrm{i}$ & ii \\
\hline & & & \multicolumn{2}{|c|}{ Player B } \\
\hline & & & invests & does not invest \\
\hline I & Dlayor & invests & $5 / 5$ & $-2 / 8$ \\
\hline II & ? & does not invest & $8 /-2$ & $-1 /-1$ \\
\hline
\end{tabular}


The Prisoners' Dilemma is one scenario that can be used to describe the incentives of different players within an economy when the usage of a public good is concerned. In reality, a situation is likely to be more dynamic because there are several influencing factors like customer demand, brand name strategy, regulation, and subsidies, and factors that are not as closely related to financial concerns like company values and mission, owners' or top management's goals, or the degree of consumer sovereignty. All these factors play a role and for a given company can result in different payoffs than the ones assumed above. If payoffs are not monetary they would represent degrees of usefulness or degrees of satisfaction. Opposing for instance to the model of the 'tragedy of the commons' by Hardin (1968), which much resembles the above example, Ostrom argues in favor of the complexity and individuality of each player's decision and each decision's context and thus criticizes simplified economic modeling (Bergstrom 2010). Her opinion was formed by examining many case studies, which showed that around the world durable institutions exist that manage common pool resources efficiently. Repeated interaction and group size were found to be important factors to develop and maintain efficient cooperation (Bergstrom 2010, Ostrom 1998). The relevance of small scale approaches suggested by Ostrom when dealing with commons or public goods, further objects the other two popular approaches of rational choice theory concerning the use of common pool resources. These are the Pigovian Approach, which argues that negative effects should be solved by imposing central regulation and/or taxation in order to align private and public interests, and the Property Rights Approach, which suggests that inefficiencies of common access problems can best be solved by eliminating public access and by assigning property rights to private owners (Bergstrom 2010).

With regard to global ecological conditions it seems that a combination of small scale solutions as suggested by Ostrom and of approaches of global governance should be pursued. Concerning the ecological effects of land use and fragmentation, whose identification, quantification, and allocation are at the center of this work, inefficiency created mostly by competition as depicted in the Prisoners' Dilemma and elevated complexity and missing interaction as depicted by Ostrom, contribute to the occurrence of the impacts. The admission of costs to reduce the impacts would lower the local and global competitiveness of a company and people who live in a city seldom are aware of the impacts on the landscape provoked by their consumption. Still less interaction occurs between the originators and the vegetative and faunal recipients of the impacts.

While competition and complexity are reasons not to engage in more environmentally friendly production, the concept of economic growth that extends beyond the productive and absorptive capacities of an area, and which serves to accumulate and preserve (the power of) capital and manifests in consumerism, is viewed to represent the 
greatest hurdle at the individual material and the collective structural level. Competition and complexity do not force mankind to live in a way it does not want to live; both are human-made. Before the nuclear catastrophe of Fukushima, competitiveness was the major reason, why in Germany nuclear energy could not be abandoned, after Fukushima it quickly could. If the complexity of global markets is overburdening the intelligence and the responsibility of the human being, then it should be avoided. While both phenomena also hold positive aspects, it appears difficult to overcome their negative consequences when they are not managed and restricted not to become dominant forces of societal organization. While not totally withdrawing competition and complexity, the economic concept of material degrowth seems promising because it seems capable of setting limits to ecological destruction and greed. In the following, the historical development of the theoretical background of the concept and a few aspects additional to the description of the concept in the main body, are given.

\section{The Concept of Degrowth}

It is still difficult to say, whether human discontent about inequality and a changed quest for self-realization and happiness or the destruction and deterioration of ecological systems and the changes in geophysical systems will lead to a change in lifestyles and economic organization. In either case, the main characteristic of change will have to be a reduction of societies' material throughput. The economic theory that deals with the question, how to achieve a socially agreeable and steady, rather than a catastrophic downshifting of an economy, is called degrowth. Transportation being a resource- and impact-intensive activity in need to be reduced itself, a drastic reduction of material throughput would significantly lower and change the demand for transport.

Degrowth is a culturally and structurally coined strategy in order for the human being to reach a form of living that at least for its hold of impact allows for a fair, which refers to the rights of all born and unborn human beings, and permanent existence under as little as possible deteriorating conditions on planet earth. As things in a living system always are subject to change, it has to be discussed what 'as little as possible deteriorating conditions' means in detail. Of the two existing concepts of weak and strong sustainability, the ecological dimension of degrowth seems to favor the concept of the latter (see section 2.3.1 and Ott \& Döring 2006 and Ott 2009b for a discussion of the concept of strong sustainability). Goergescu-Roegen points out that the maintenance of entirely non-deteriorating conditions is impossible because the stock of terrestrial low entropy is limited and because the balance of any biological or economic activity is always negative, which means that it results in higher entropy that contains more bound and less free energy. Bound energy humans can no longer use. Entropy is a measure of unavailable energy in a thermodynamic system. Goergescu-Roegen ex- 
plains that what is regarded economic development, that is substituting an ox for a tractor or manure for artificial fertilizer changes free into bound energy and hence is antieconomical because it uses up more scarce low entropy. The production of a car destroys low entropy that is no longer available for the production of a spade. This decreases the number of future human lives because besides asking how many people planet earth can sustain in one year, the more relevant question seems to be how long a given world population can be maintained. The less humankind uses power from solar radiation, which is the only other source of energy and which so far is mostly be used by obtaining mechanical power from using chlorophyll photosynthesis for the nutrition of living organisms, the more it shortens its species life span as solar radiation is expected to be available much longer than the stock of terrestrial low entropy (Goergescu-Roegen 1973).

While entropy was not adopted as the measuring unit in degrowth research, the reason maybe being that it would be too discouraging as mankind had become too dependent and enthusiastic on the gains of development which in turn would make it a task too difficult and too lengthy to be considered philosophically, Goergescu-Roegen after the publication of his book 'Demain la decroissance' in 1979 still became a leading figure in the degrowth movement. The original and still primary goal of degrowth is to establish an economy of a stable material throughput that does not exceed ecological limits. Since its introduction by Mill in 1857 (Bladen \& Robson 1965), this was the dominant strain referred to for more than a century, inter alia, by Goergescu-Roegen (1979) and Daly $(1973,1977)$. In the more recent past, a second dimension of and motivation for degrowth, which is maybe best described as a cultural or philosophical strain, became meaningful (Latouche 2010, Schneider et al. 2010, Kallis 2011, Paech 2012a). It mainly consists of the perceptions that praising the development model of the USA and Europe is wrong, that short-term economic interest undermines democracy, and that growth does not increase happiness or allow living flourishing lives characterized by self-actualization, wealth of time, relatedness, and non-violence, also with regard to non-human life forms.

The normative goal of degrowth is not to degrow GDP, but to reduce material throughput in a socially desirable way. While this demands for qualitative improvements in economic, social, and cultural spheres, GDP nonetheless is expected to decrease due to an organization of smaller regional economies in which people adopt dematerialized, less pecuniary ways of living to some degree. While the reasons for which degrowth is viewed necessary to some extent suggest what resulting societies may look like, the focus of the concept is on getting underway and on shaping the process of how a significant reduction in throughput and changes in structures and lifestyles can be achieved in a non-disruptive and socially supported way. 
In 2013, the earth overshoot day, when the human ecological footprint surpassed the biocapacity of the earth was on August 20 (GFN 2013). At the end of the year, human demand and impact reached a level that 1.76 planets of the type of the earth could sustain. In order to picture responsibilities fairly, it must be said that high income countries (World Bank classification) have a much higher impact. Already in 2007, their footprint was 1.99 times the size of the biocapacity of the earth (GFN 2010). While this does not mean that industrialization must be reversed, two conclusions can be drawn: firstly, that many of the technological gains and gains in global purchasing power need to be treated and enjoyed more responsibly, particularly with regard to quantity, and secondly, that for the technological advances and the already realized construction of buildings and infrastructure, industrialized societies' impact should rather be below the average sustainable impact. This means that the concept of degrowth does not apply to the people and the countries of the global South, who besides being given the help they might ask for, are free to define their own trajectory.

The situation depicted above shows, how growth of consumption is metabolically unsustainable. This applies to the generation of energy, the availability of resources, and the functioning of ecological and atmospheric systems. The hypothesis to decouple environmental damage from economic growth due to the benefits obtained from technological innovation and gains in efficiency has proven to have been false in the past and is not likely to come true in the future. One reason is that unless valued differently savings tend to rebound, which can make the total balance of throughput after innovation negative (Jackson 2009, Paech 2012a).

Although the purely anthropocentrically reasoned, resource-orientated ecological dimension is more difficult to argue with, the before mentioned second cultural and philosophical dimension arguing for degrowth due to its relevance in the recent past has received increasing attention. In his book 'Farewell to growth' Latouche (2010) concisely names three reasons that oppose growth from this perspective: firstly, growth did not increase happiness, secondly, credit, advertising, and planned obsolescence maintained a treadmill of production, consumption, and growth for growth's sake, binding the creativity, potential for relatedness, and personal quest for the meaning of live, and thirdly, the idea of development as growth was corrupting non-Western cultures. Regarding the latter, Baudrillard (2005) identified non-Western cultures' aspiration for Western lifestyles to be the only thing that kept growth economies going. With regard to happiness and peace, Illich (1978) viewed the competitive and impersonal character of money and markets to be a significant threat; and growth needed markets, or war. Illich further stated that in a market intensive economy, what mattered was not the effort to achieve happiness and enjoyment, but best to couple labor with capital. Already in his former and much discussed work 'Tools for conviviality', Illich said that capital held mo- 
nopolies on basic human activities such as health, agriculture, or learning, which threatened subsistence and conviviality (1973). He further claimed that economic development often did not result in human flourishing, but in modernized poverty and dependency. In his historical analysis on money and debt, Graeber (2011) opines that the theological fall of man and the subsequent punishment by self-love, by Hobbes was changed to the supposition that the human being was not content with a certain degree of welfare, but for its self-interest would want to increase its pleasure and accept competition. This moral component together with the legalization to demand interest, led to a change in economic order substituting practices of sharing and credit for the maxim of interest. With regard to sociability, responsibility, and subsequently happiness, Graeber considers the ability of money to turn moral into an impersonal arithmetic that legitimizes actions that would otherwise be disdained, as most harmful. While potential negative consequences of immoral conduct are most hurtful at the receiving end, the aspect of reduced relatedness also is harmful for the other party considering the result of Becker's analysis of the human being to be significantly relational.

Mainly because the characteristics of self-interest and avarice were unable to result in peace and a certain degree of egality and led to a transcending from nature, missing relational experiences in consumption, and a reduction of self-esteem to the strength in competition, all of which center on having or possessing things and power instead of being solidary and in comforting relations, Fromm claimed that the health of a competitive market system could only be attained for the sake of sick people (Fromm 1976). His assessment drastically summarizes the second strain of motivation inherent to the concept of degrowth discussed today. Okri (2008, p. 1) refers to it as he formulates: "We must bring back into society a deeper sense of the purpose of living. The unhappiness in so many lives ought to tell us that success alone is not enough. Material success has brought us to a strange spiritual and moral bankruptcy". Yet, the majority of people today tend to aspire to define themselves by what they have. Under this perception, to live more simply, for instance by renouncing material possessions, is a threatening prospect because it means "a partial or total loss of identity, power, privilege and self" (Burch 2009, p. 192). To conclude the discussion of the two strains of motivation for degrowth, it is assessed that despite the significance of the realization and the related, increasingly attended moral implications of the earth to be limited in the provision of resources, the perceptions that happiness, self-development, and peace can better be attained in gathering less power and possessions and in perceiving the restrictions from simple living rather to be pleasing sources of solidary relation appear to be of higher importance for material societal throughput to be reduced voluntarily.

Regarding a potential implementation of the concept, the most important principles and political policies that can help to get degrowth underway were presented in detail in 
section 2.3.2. Some of the principles that individual policies are aimed at are a multilevel and more direct democracy, a redistribution of work, leisure, natural resources, and wealth, and a re-localization of the economy. Taxes on environmentally damaging activities or the introduction of regional currencies would be two policies that would directly concern transportation. With regard to key elements, for Paech (2012a, 2014) the principles of subsistence and sufficiency are the fundament of post-growth economies because existing structural driving forces for growth, inter alia, could be reduced by shortened chains of financing, production, and consumption, and present cultural driving forces would abate when understanding reduced consumption as liberation and enrichment instead of renunciation, meaning a change in behavior to be free from coercion (Stengel 2011). For instance would subsistence lower the demands of capital, if non-pecuniary local provision and regional trade using interest-free regional currencies increased and necessary global produce decreased. 9

The idea of redistributing political power and wealth is likely to meet the resistance of those who have most to lose, which according to Kempf (2010) and Burch (2009) is one significant reason for these actors to prospect increases in welfare to be attained by growth because they do not favor the alternative of reallocation. While it remains uncertain, if, and how exactly the depicted severe social and political transition would happen, Latouche (2010) at this point in time views it sufficiently important to challenge the imaginary that growth was a prerequisite for happiness and economic organization. His work aimed to set out the vision that people individually and as a society could live better with less, and that a difficult, but desired and self-determined transition might be preferable to politically or environmentally forced, more drastic change. Expanding on an above assessment, the perception of the individual that due to the human being's partially relational character, from a certain threshold onward, having less can allow 'being more' because sharing is an act of solidarity and frees capacities for interaction, appears to be the most powerful and most interesting to observe lever to enable a post-growth transition.

While the concept of degrowth is unlikely to be societally and politically adopted in the immediate future, it neither is exceedingly far-fetched because singular post-growth policies are already being implemented in different countries and because local initiatives exist that, inter alia, seek to reduce environmental impact and to enhance com-

9 The concept of subsistence economy refers to the survival of groups that live by a minimal dependency on markets. In a non-monetary economy people use local natural resources to provide for their basic material and immaterial needs. There is no impetus for profit, but communication, participation, and cooperation, which consistently reproduce on a basis of mutual responsibility, build the foundation (Dahm 2003). 
munal structures (see section 2.3.3 for more details). Initiatives and ideas differ in profundity of implementation and development. Predominantly, they are purposefully adopted, developed, and vocalized by people who are no professional politicians. This triggers and renders possible high-level political attention and action. It seems that for a transition to occur, incitements both from local activity and national political action are necessary. While with regard to logistics, a few new requirements for regional logistics would arise, the expected overall impact from reduced consumption and increased regional subsistence would be a decrease in gross national haulage, with the respective positive ecological consequence of lowered impact. The developed indicators can be used better to understand the impacts of land use and fragmentation and to apprehend certain behaviors that decrease individual impact potentially contributing to move society in the direction of ecologically sustainable economics.

\section{Consumer Sovereignty}

Consumer sovereignty is an important lever to trigger sustainable activity in companies because in spite of ecological and ethical responsibility to be held by all players along a supply chain, demands provoke production and because the decision of consumers has a function similar to a final verdict as to the adequacy of implications. Vicky Robin put it: "How we spend our money is how we vote on what exists in the world" (Alexander 2009, p. 9). Consumers usually feel a certain degree of responsibility because they sense to be influential when purchasing or not purchasing an item. However, the degree to which capability and responsibility are felt depends on various conditions. Although businesses and some researchers still view consumer demand as the most powerful trigger toward gradually integrating more ecologically sustainable practices into business processes and products (AmCham 2011, Berger 2012), in literature a discussion on the current state of consumer sovereignty can be found (Henry 2010).

The saying that "the consumer is the mother of all dictatorships" unequivocally refers to a strong influence of consumers. Yet, it can be argued that today's complex and global production systems, as well as the inducement of advertising, do not allow for consumers' decisions to be informed and morally reflected well enough adequately to speak of sovereignty anymore (Goodwin et al. 2010, cp. Becker in section 2.2.3). A second critical variable is pricing. Besides reinforcing the alienation from production processes, globalization has fostered mass production and specialization. For many products, this drove down production costs to a degree that regional products and products that are made attaching great importance to a sustainable life cycle cannot to compete with. The result is a more than marginal difference in prices, which is noted by consumers. However, the major share of consumers in Western countries possesses the economic requisite to make purchasing decisions, in which they do not value pric- 
ing as the pivotal criteria. In summary, it is viewed legitimate to contend, that a lack of information threatens the sovereignty of consumers, but that differences in product pricing, while favoring cheaper products, do not disable consumer sovereignty. Hence, the sovereignty over one's personal values and consumptive choices still make for consumers potentially to be a powerful trigger of production. Usually, the influence of consumers is much stronger in business-to-consumer than in business-to-business relationships because the impact of a final consumer's values and purchasing decisions abates downward the supply chain. It is viewed that in the way of spending their money, consumers have not lost their capability to vote on the form of sustainability that they want to see to be practiced in the world.

\section{Capital Sovereignty}

Capital sovereignty here refers to the situation in existing market economies, where all basic institutions, such as financial, property, political, and redistributive, depend on and mandate economic growth (Kallis 2011). In addition to the link between growth and logistics and business economics, capital sovereignty refers to the structural democratic and proprietary implications of growth and capital distribution. A concentration of capital was found to be risky and likely to be harmful to commons and the environment. The use of private property as collateral (van Griethuysen 2010), debt and the interestearning system (Paech 2009), and the grow-or-die competition of private enterprises for profit and market share (Kovel 2002), make growth a structural feature of capitalism that facilitates capital sovereignty (Harvey 2007). Some critics say that the capitalist growth model failed not only in environmental or social terms, but also in its own terms of maintaining growth (Korten 2009). Most renown theorists like Marx, Weber, Schumpeter, or von Mises, independent of their position in the political spectrum, expected capitalism to last for one, or at most two generations (Graeber 2011). In order to protect surplus, capitalism in the past was extended beyond the real economy and a process well described as financialization is happening until today. Financialization is the conversion of public and private assets, such as social systems, land, water, education, mobility, patents, information, police, or the planet, to the holders of financial products. This can be considered unegalitarian to a degree that conflicts with human rights. For instance in the case of information, the gathering of data cannot only be a strategy to protect ongoing economic imperialism, it also is repressive, undemocratic, and breaking with the human right of freedom of speech. In neoliberalism, Kempf (2010) and Foster (2013) see the end of liberal democracy and rather a condition of oligarchy. With respect to capital, Foster (2013) sees the disappearance of relative autonomy of the state, which transfers sovereignty from the people to capital. That in a new macroeconomic model, which could follow the steady-state economy of Daily, in which mate- 
rial throughput lies within the regenerative capacities of ecosystems (Daily 1996), the organization and functioning of financial institutions as major capital holders and as today's fuses of partially perverse activity would have to undergo considerable change, even one of the most recognized free-market advocates, Paul Krugman might accept as he admits: "People that questioned the belief in financial markets were up against a pervasive and, in retrospect, foolish complacency" (Krugman 2009, p. 1). This concludes the more thorough analysis of current economic circumstances that are met by the implementation of the concept of sustainability and that allow assessing the potential of the here developed methods of quantifying ecological impact. 
C Map of the Federal States of Germany

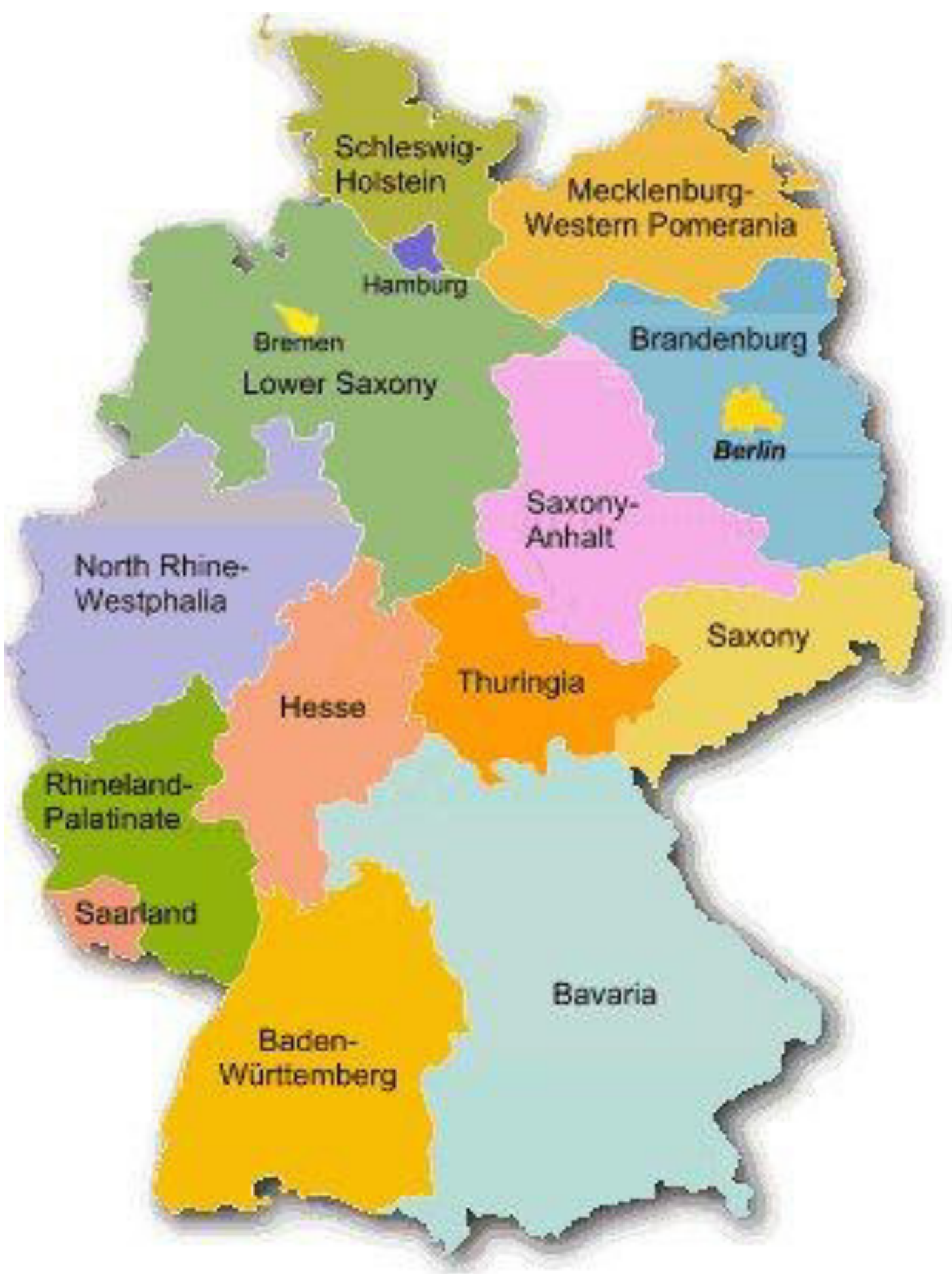

Figure Ap.1: Map of the federal states of Germany; source: Ancestry 2009. 


\section{Traffic Volume Impacts on Wildlife at Different Thresholds}

Table Ap.2: Traffic volume impacts on wildlife at different thresholds; source Charry and Jones 2009.

\begin{tabular}{|c|c|c|c|c|}
\hline Vehicles/Day & $\begin{array}{l}\text { Onset of } \\
\text { impacts }\end{array}$ & $\begin{array}{l}\text { Continuum of } \\
\text { substantial impacts }\end{array}$ & $\begin{array}{l}\text { Major } \\
\text { habitat } \\
\text { avoidance }\end{array}$ & $\begin{array}{l}\text { Near } \\
\text { complete } \\
\text { barrier }\end{array}$ \\
\hline $100-500$ & $\begin{array}{l}\text { amphibians, } \\
\text { carnivores }\end{array}$ & amphibians, reptiles & & \\
\hline $500-1,500$ & $\begin{array}{l}\text { ungulates, } \\
\text { birds }\end{array}$ & $\begin{array}{l}\text { amphibians; increases } \\
\text { for reptiles }\end{array}$ & & \\
\hline $1,500-3,000$ & & $\begin{array}{l}\text { Ungulates; increases } \\
\text { for amphibians \& rep- } \\
\text { tiles }\end{array}$ & & \\
\hline $3,000-6,000$ & & $\begin{array}{l}\text { carnivores, birds; in- } \\
\text { creases for amphibians, } \\
\text { reptiles \& ungulates }\end{array}$ & & \\
\hline $6,000-10,000$ & & $\begin{array}{l}\text { increases for amphibi- } \\
\text { ans, carnivores, ungu- } \\
\text { lates \& birds }\end{array}$ & & reptiles \\
\hline $10,000+$ & & & $\begin{array}{l}\text { birds, un- } \\
\text { gulates }\end{array}$ & $\begin{array}{l}\text { amphibians, } \\
\text { reptiles, carni- } \\
\text { vores, ungu- } \\
\text { lates, small } \\
\text { mammals }\end{array}$ \\
\hline
\end{tabular}




\section{E Maps of the Two Exemplary Routes}

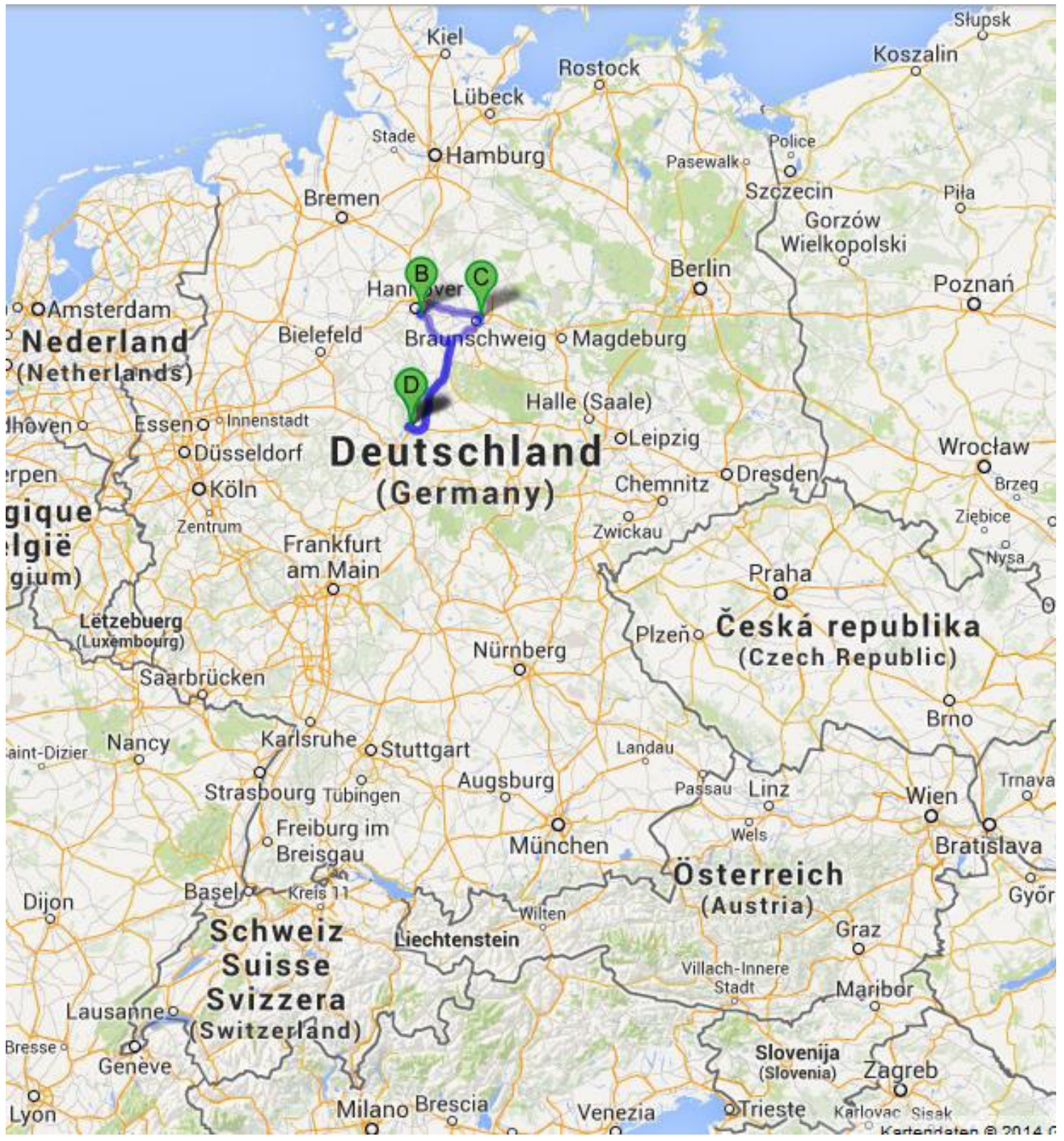

Figure Ap.2: Route display Hann. Münden to Hannover to Brunswick and back to Hann. Münden; source: Google Maps 2014b. 


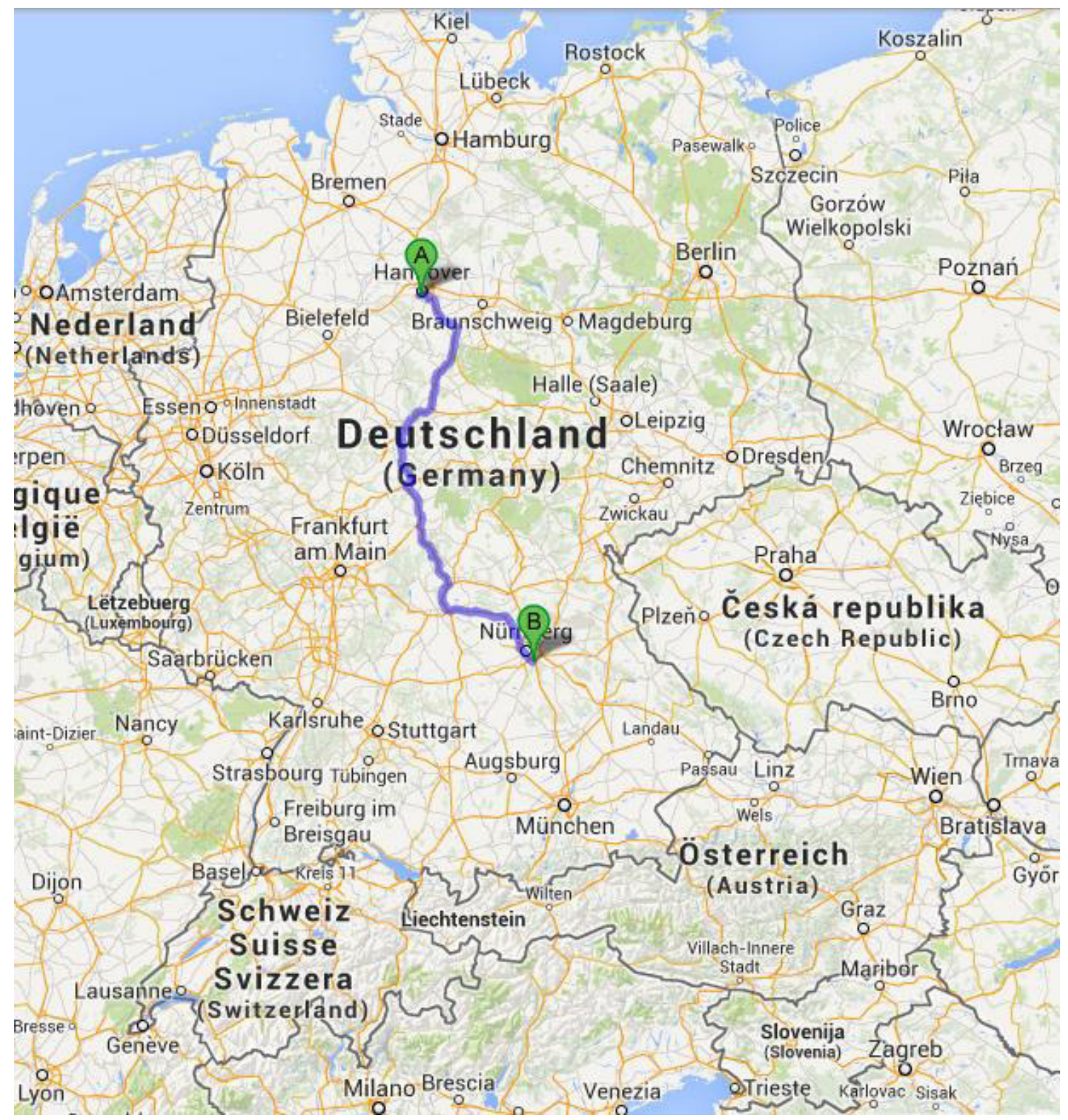

Figure Ap.3: Route display Hannover to Wendelstein; source: Google Maps 2014c. 



\section{ARTICLE I}

Friedrich, J., and J. Geldermann. 2013a. Potentielle Auswirkungen von Straßenverkehrstransporten auf die Biodiversität. In Treffpunkt Biologische Vielfalt 12: Interdisziplinärer Forschungsaustausch im Rahmen des Übereinkommens über die Biologische Vielfalt , eds. U. Feit and H. Korn, pp. 107-113. Bonn: Federal Agency for Nature Conservation.

Printed with permission for non-commercial use. 



\title{
Potentielle Auswirkungen von Straßenverkehrstransporten auf die Biodiversität
}

\author{
JAN FRIEDRICH \& JUTTA GELDERMANN
}

\section{Schagwörter: Flächeninanspruchnahme, Landschaftszerschneidung, Straßenverkehrstransport, Biodiversität; Umweltmanagement}

Der Energie- und Ressourcenverbrauch der sogenannten entwickelten Welt übersteigt die lokalen und globalen natürlichen Kapazitäten. Er ist der maßgebliche direkte Grund für die verschiedenen Ausprägungen der ökologischen Krise. $\mathrm{Zu}$ deren wesentlichen Ausprägungen zählen der Klimawandel, der Süßwassermangel, das Artensterben, oder die Übernutzung und Verschmutzung von Böden, Wasser und Luft. Ökologische Knappheiten und Probleme bestehen dabei nicht an sich, sondern sie werden von sozialen Knappheiten, also vom Verhalten und von den Lebensstilen der Menschen, hervorgerufen. ${ }^{1}$ Dies ist auch für das Spannungsfeld Verkehr und Umwelt der Fall. Die heutige Transportintensität westlicher Produktionsstrukturen und Konsumarten kollidiert signifikant mit den Bedürfnissen anderer Lebewesen. Vergleichbar mit den negativen Effekten von Verkehr für den Menschen, wie Lärm an Flughäfen und Bahntrassen oder Emissionen in Städten, sind auch zahlreiche Tierarten gerade vom dominierenden Kraftwagenverkehr auf Straßen gestört oder in einigen Fällen gar in ihrer Existenz gefährdet. Die für Tiere und Pflanzen negativen Effekte sind weitestgehend bekannt und gewinnen im Zuge der politischen Bemühungen zum Schutz der biologischen Vielfalt nun auch auBerhalb der Naturwissenschaften an Bedeutung. Ziel dieses Beitrags ist es, betriebswirtschaftlichen Entscheidern Informationen und methodische Hilfsmittel zur besseren Berücksichtigung und zur Verringerung der potentiellen negativen Auswirkungen von Straßentransporten zu liefern.

Dazu werden die Ergebnisse folgender beider zentraler Fragestellungen präsentiert:

1. Welche maßgeblichen potentiellen negativen Effekte von Straßenverkehrstransporten auf die nicht menschliche Natur bestehen?

2. Kann für die beiden Effekte Flächeninanspruchnahme und Landschaftszerschneidung eine Operationalisierung und Quantifizierung gefunden werden, die unternehmerischen Entscheidern bei der Verminderung dieser Effekte hilft?

Grundlage der beiden Fragestellungen ist eine Lücke zwischen der Berücksichtigung des Themas in Unternehmen sowie ihrem Umfeld und den Ergebnissen der naturwissenschaftlichen Disziplin StraBenökologie. Zwar befassen sich Unternehmen, gesetzliche Verordnungen und Ökobilanzen verstärkt mit der Ökologieverträglichkeit von industriellen Produktionsprozessen, dennoch hat die Informationstiefe sowie das Interesse in der Breite erst in jüngerer Zeit ein signifikantes Ausmaß angenommen². Beispielsweise gibt es schon seit Jahrzehnten Zertifizierungen wie den Blauen Engel (Einführung 1978) oder Regularien wie die Umweltverträglichkeitsprüfung (1990). Die Entwicklung in der Ökobilanzierung (Eco-Indicator von 1999; CML-Modell von 2001), bei der Nachfrage nach Umweltmana-

1 vgl. ULRICH 1991, S. 2

2 vgl. GUINÉE et al. 2002 
gementsystemen (Eco-Management Audit Scheme - EMAS, International Organization of Standartization - ISO, Global Reporting Initiative - GRI) und die Bedeutung der Nachhaltigkeitsberichterstattung in Unternehmen wurde vor allem auch im letzten Jahrzehnt dynamischer.

Mit dem globalen Phänomen des Verlusts von Biodiversität, der sich in der Schädigung von Ökosystemen und in einem Rückgang der Vielfalt an Arten und Genen zeigt, stellt sich der Gesellschaft und somit auch den Unternehmen eines der bisher komplexesten ökologischen Probleme. Trotz Veröffentlichung eines Meilensteinberichts zur Bewertung des Zustands und der Gefahren für die biologische Vielfalt durch das Millennium Assessment in 2005, bedurfte das Thema Biodiversität der breiten öffentlichen Diskussionen zum Klimawandel und folgte so in der politischen, wissenschaftlichen und öffentlichen Wahrnehmung erst auf die Phase nach dem Erscheinen des vierten IPCC Sachstandsberichts in $2007 .^{3}$ So zeigten beispielsweise Schwerpunktthemen in Gesprächen mit Unternehmensvertretern, Inhalte der mit EU Life+ Mitteln geförderten und von einigen NGOs geführten Business \& Biodiversity Campaign und die Analyse von Nachhaltigkeitsberichten und Umweltmanagementprogrammen, dass die meisten Unternehmen im Transportbereich nahezu ausschließlich $\mathrm{CO}_{2}$-äquivalente Emissionen als signifikante ökologische Belastung ansehen. Es gibt verschiedene Bemühungen, wie den Einsatz von Leichtlaufreifen, Fahrerschulungen oder eine schrittweise Erneuerung des Fuhrparks, um den Ausstoß von transportbedingten Emissionen zu verringern. Diesem Vorgehen stehen Erkenntnisse der Straßenökologie über weitere signifikante Auswirkungen von Verkehr gegenüber. Straßenökologie kann definiert werden als die von Straßen und Fahrzeugen beeinflusste Wechselwirkung zwischen Organismen und ihrer Umwelt. ${ }^{4}$ Das Ziel des Fachgebiets, die Umweltauswirkungen von Straßen systematisch zu erfassen und zu bewerten und so eine Voraussetzung für die Verringerung von negativen Effekten zu liefern, wurde in den 1970er- und 80er-Jahren maßgeblich durch Arbeiten von Mader und Ellenberg vorangebracht. ${ }^{5}$ Mit dem Gesamtwerk Road Ecology von Forman et al. in 2003 und durch zahlreiche weitere Arbeiten, wurden weitere Fortschritte über die Kenntnis der Beschaffenheit der einzelnen Auswirkungen von Verkehr erzielt. ${ }^{6}$ Aus dieser nicht kongruenten Situation der beiden Bereiche leitet sich das Ziel der Integration straßenökologischer Erkenntnisse in betriebswirtschaftliche Nachhaltigkeitsbemühungen ab.

Dazu sind in einem ersten Schritt die maßgeblichen potentiell schädlichen Auswirkungen zu identifizieren, bevor im zweiten Schritt eine Instrumentalisierung der beiden Effekte Flächeninanspruchnahme und Landschaftszerschneidung vorgenommen wird. Methodisch wird dabei in Teilen auf die beiden in der Wirkungsanalyse von Mensch-Umwelt-Systemen etablierten Ansätze des DPSIRModells und der ökologischen Risikoanalyse zurückgegriffen. ${ }^{7}$ Die Identifikation der Beeinträchtigungen entspricht dabei der zweiten Phase des DPSIR-Modells. Dieses wurde von der Europäischen Umweltagentur (EEA), auf Basis des zum Ende der 1980er Jahre von der OECD entworfenen PSRModells, weiterentwickelt. ${ }^{8}$ Ziel war es, bei der Darstellung von Umweltbelastungen und Schutzmaßnahmen einen Systemansatz zu schaffen, der die Probleme und die Wirkungen möglicher Lösungsan-

3 vgl. MiLlENIUM ECOSYSTEM ASSESSMENT 2005; vgl. IPCC 2007

4 vgl. FORMAN et al. 2003

5 ROEDENBECK UND JAEGER 2006, S. 297

6 vgl. FORMAN et al. 2003; RECK et al. 2005; JAEGER et al. 2005; vgl. FAHRIG 2003

7 DPSIR steht dabei für driver (Treiber), pressure (Belastung), state (Zustand), impact (Auswirkung) und response (Reaktion).

8 OECD 1993, S. 5; EEA 2003, S. 13 
sätze als kausale Kette und vor allem unter Berücksichtigung der begleitenden Einflüsse sozioökonomischer Entwicklungen abbildet. Als ökologische Belastungen durch Straßengütertransporte können folgende sechs wesentliche Einflüsse identifiziert werden:

1. Die Flächeninanspruchnahme und -versieglung durch Verkehrsinfrastruktur sowie die Degradierung anliegender Gebiete zerstört natürliche Fläche. Auch wenn der Begriff Flächeninanspruchnahme dem Terminus Flächenverbrauch vorzuziehen ist, weil er offen lässt, dass genutzte Fläche theoretisch auch renaturiert werden kann, gehen mit der Versiegelung Flächen, die zuvor Pflanzen und Tieren als Habitate zur Verfügung standen und Ort von produktiven Ökosystemen mit Bodenfruchtbarkeit, Sauerstoffproduktion und Dekomposition waren, zumeist unwiederbringlich verloren. Durch stoffliche Emissionen ebenso wie durch immaterielle Emissionen wie Licht und Lärm und auch wegen der Auswirkungen auf das Lokalklima in Form von veränderter Luftfeuchtigkeit, veränderter Temperatur oder veränderter Wasserflüsse werden auch anliegende Gebiete in ihrer natürlichen Qualität beeinträchtigt.

2. Belastend sind auch der Vorgang des Zerteilens von Populationen durch lineare Infrastruktur sowie die Barrierewirkung von viel befahrenen Straßen, auf Grund derer Individuen den Zugang zu Ressourcen, Ruheplätzen, saisonalen Habitaten, Partnern und/ oder zu entfernten Gebieten verlieren. Neben den direkten Einschränkungen und Gefahren sowohl für Individuen als auch für die Gruppe, ist für den langfristigen Fortbestand einer Population in besonderem Maße die sich mit der Zeit einstellende kleiner werdende Variabilität des Genpools problematisch. Denn sie bedeutet einen Rückgang der Stabilität von Populationen insgesamt, darunter fällt zum Beispiel eine geringere Anpassungsfähigkeit an veränderte Umweltbedingungen, wie sie etwa durch ein Verschieben von Lebensräumen durch den Klimawandel auftreten. Die zunächst schlimmste Ausprägung dieses Effektes wäre Inzucht. Doch schon eine geringe, kaum sichtbare und schwerlich reversierbare verringerte Variabilität des Genoms stellt eine für eine Population höchst bedrohliche Entwicklung dar, weil sie das Extinktionsrisiko erhöht.

3. Da nicht jede Straße auf Tiere wie eine Barriere wirkt, sondern dies vor allem von der Anzahl und der Verteilung der Fahrzeuge im Tagesverlauf sowie dem Verhalten der jeweiligen Art abhängt, versuchen Tiere häufig Straßen zu überqueren. Aufgrund des Zusammenstoßes mit Fahrzeugen kommt es so signifikant häufig zu Mortalität. Betroffen sind viele Tierarten, darunter Kröten, Insekten, Vögel, Hasen, Igel, Rehe, Otter oder Wildkatzen. Bei den beiden letztgenannten war der Tod durch Kollision mit einem Fahrzeug zeitweise die häufigste Todesursache. ${ }^{9}$ Die in starken Konfliktzonen angewandte Lösungsmöglichkeit von Straßenbezäunung zeigt, dass die einzelnen Auswirkungen mitunter stark miteinander verknüpft sind. So kann Bezäunung zwar das Mortalitätsproblem vermindern, jedoch ebenfalls die Zerschneidungswirkung erhöhen und somit das Gesamtproblem der Auswirkung der Straße eher verlagern als lösen.

HERRMANN et al 2007, S. 3 
4. Beim Betrieb brennstoffbetriebener Kraftwagen auf Straßen fallen eine Reihe stofflicher Austräge an, die die natürliche Umwelt schädigen können. Zu nennen sind hier die auch für den Menschen relevanten Luftschadstoffe wie Feinstaub, Ozon, Kohlenmonoxid, flüchtige organische Verbindungen und Stickoxide. Weitere stoffliche Austräge fallen durch Gummiabrieb und Gummiteile, Streusalze oder durch die Entsorgung von Abfällen an. Wegen der globalen Bedeutsamkeit des Ausstoßes von Treibhausgasen wird der Ausstoß von $\mathrm{CO}_{2}$-äquivalenten Emissionen, im Verkehr fast ausschließlich Kohlendioxid (96,75\% $\left.\mathrm{CO}_{2} ; 2,3 \% \mathrm{~N}_{2} \mathrm{O} ; 0,95 \% \mathrm{CH}_{4}\right)$, hier gesondert aufgeführt. Der Anteil der vom Verkehrssektor ausgestoßenen Emissionen an den globalen Gesamtemissionen lag 2004 bei 13,1\%, für den Straßenverkehr allein bei $9,9 \%{ }^{10}$

5. Transportmittel lösen häufig die Problematik invasiver Arten aus oder verschärfen diese. Als invasive oder gebietsfremde Arten werden Pflanzen und Tiere bezeichnet, die durch menschliche Einwirkung in ein Gebiet eingebracht werden, in dem sie natürlich nicht vorkommen. Diese Arten werden dann problematisch, wenn sie die biologische Vielfalt gefährden, indem sie zum Beispiel aufgrund ihrer überlegenen Konkurrenzfähigkeit einzelne heimische Arten verdrängen oder ganze ökologische Teilsysteme gefährden. ${ }^{11}$

6. Nicht zuletzt sind auch Einflüsse, die nicht vor Ort auftreten oder nicht vor Ort verursacht werden, dennoch aber der Nutzung von Fahrzeugen zuzuschreiben sind, zu berücksichtigen. Dies umfasst die Auswirkungen des gesamten Lebenszyklus der beiden Komponenten Infrastruktur und Fahrzeug, von der Ressourcengewinnung, dem Bau bzw. der Produktion bis zur Wiederverwertung oder Entsorgung. Ausgenommen ist die Nutzungsphase, deren spezifische potentielle direkte Belastungen oben bereits einzeln betrachtet wurden.

Auf die Identifikation der potentiell negativen Effekte folgt nun die Instrumentalisierung der beiden Belastungen Flächeninanspruchnahme und Landschaftszerschneidung. Die Analysemethode lehnt sich nun stärker an das Konzept der ökologischen Risikoanalyse an. Leitidee dieses Verfahrens ist eine Verknüpfung der Intensität potentieller Belastungen mit der Beeinträchtigungsempfindlichkeit des ökologischen Systems, deren Ergebnis das berechnete ökologische Risiko ist. So kann eine Belastung am Ende nicht nur als signifikant oder vernachlässigbar bewertet werden, sondern muss sehr detailliert betrachtet werden. Ziel der Methode, die Ende der 70er Jahre maßgeblich von Bachfischer entwickelt wurde, ist die Beurteilung der ökologischen Verträglichkeit von Nutzungen bei unvollständiger Information. Dem Ansatz liegt dabei kein klar definiertes Bewertungsverfahren zugrunde. Ein Indiz dafür könnte sein, dass die Methode den wirtschaftlichen Triebkräften der Politik sowie dem Vorwurf der mangelnden planerischen Integrierbarkeit von langfristigen natürlichen und sozialen Ressourcen ein handfestes Argument entgegensetzen sollte und es somit zunächst eher auf die Leitidee der Analyse

10 IPCC 2007, S. 36

11 Hubo et al. 2007, S. 95 
ankam. ${ }^{12}$ Die beiden unten vorgestellten Quantifizierungsmodelle sind ebenso wie die ökologische Risikoanalyse nicht frei von Werturteilen. Ein weiterer Diskurs ist deshalb sinnvoll.

Für die Flächeninanspruchnahme besteht der Ansatz darin, sowohl für die faktisch verbrauchte, als auch für die anliegende degradierte Fläche, den Anteil eines einzelnen Nutzers zu berechnen. Der Zeithorizont beträgt ein Jahr, da Unternehmen in der Regel jährlich ihre Leistungen bewerten. Zudem kann von einer Nutzungsänderung nach einem Jahr bereits ein signifikantes Signal an die Raum- und Verkehrsplanung ausgehen, ob zusätzliche Kapazitäten benötigt, der Status Quo erhalten werden, oder ein Rückbau von Verkehrsinfrastruktur stattfinden soll. Die Berechnung der versiegelten Fläche erfolgt über die Anzahl der Fahrstreifen einer Straße. Unter Einbezug unter anderem von Rand- und Mittelstreifen, Rastplätzen und Auf- und Abfahrten wurde ein Durchschnittswert von 5,5 Metern pro Fahrstreifen ermittelt. Zur anteiligen Berechnung werden Daten der Verkehrsintensität benötigt. Eine Strecke teilt sich in verschiedene Abschnitte, wenn eine der beiden Zahlen sich ändert. Bei der degradierten Fläche werden Bereiche von 2 km rechts und links der Fahrbahn mit nachlassender Beeinträchtigung bei zunehmender Entfernung vom Straßenkörper und in Abhängigkeit vom Verkehrsaufkommen für die anteilmäßige Berechnung angenommen. Bei der Quantifizierung der Zerschneidung verwendet der Indikator Ergebnisse einer Arbeit, in der GIS gestützte Karten für wichtige Wildtierwanderkorridore erstellt wurden und in der anschließend mithilfe digitaler Daten für das deutsche Straßennetz Konfliktstellen unterschiedlicher Schwere ausgewiesen werden konnten. ${ }^{13}$ Daran angelehnt addiert der hier entwickelte Indikator die Zerschneidungskonflikte einer Strecke in Abhängigkeit ihrer Schwere und anteilig an der Gesamtzahl von Nutzern innerhalb eines Jahres. ${ }^{14}$

Wichtig zu erwähnen ist, dass die hier beschrittene umwelttechnische Analyse nur eine Teilantwort auf die ökologische Frage ist. $\mathrm{Zu}$ ihrer gesamten erfolgreichen Beantwortung ist eine Analyse des sozialökologischen Kontexts mit Reflektion der gesellschaftlichen und politischen Rahmenbedingungen ebenso notwendig. ${ }^{15}$ Neben der ökologischen und der sozialen Dimension stellt sich der Organisation unserer europäischen Gesellschaft im Status Quo zentral auch eine wirtschaftspolitische Frage: Wie kann ein auf nicht weiter möglichem Wachstum beruhendes Sozial- und Wirtschaftssystem auf den Level eines ökologisch nachhaltigen und global sozialgerechten Systems angehoben werden, ohne chaotische Zustände bei der Beschäftigung und der Versorgung der Menschen, und ohne den Verlust lebensbereichernder Errungenschaften, zum Beispiel im Gesundheitswesen oder in der Mobilität, hervorzurufen? Allein mit technischen Errungenschaften und Effizienzverbesserungen lässt sich der momentane Level des Verzehrs ökologischer Ressourcen vor allem westlicher Lebensstile, weder für die heutige, noch die in Zukunft größere Anzahl an Menschen aufrechterhalten. ${ }^{16}$ Da ein etliche Ebenen betreffender Wandel kaum von den im jetzigen Modell erfolgreichen Institutionen und Akteuren ausgehen wird, liegt Potential vor allem bei den einzelnen Menschen aufgrund bewusster oder veränderter Wertvorstellungen zu weniger ressourcenintensiven und suffizienteren Lebensstilen überzugehen. ${ }^{17}$ Der Soziologe Oliver Stengel sieht in der ,auf die Verringerung des Güterkonsums zielenden

12 Aulig et al. 1977, S. 28

13 HÄNEL et al. 2010

14 Aufgrund des begrenzten Umfangs dieses Beitrags werden die beiden Formeln nicht abgebildet oder detaillierter erläutert. Ein Aufsatz in dem dies geschieht erscheint in Kürze im Journal Oecologia Australis.

15 vgl. ULRICH 1991

16 vgl. MEADOWs et al. 2004; vgl. SEIDL UND ZAHRNT 2010

17 vgl. LATOUCHE 2009 
Suffizienzstrategie eine unumgängliche Strategie zur Minderung der sich abzeichnenden und teilweise bereits ereignenden Umweltgefährdungen“. ${ }^{18}$

Unabhängig von den Entwicklungen in anderen Themenbereichen versuchen die hier vollzogene Identifikation potentieller negativer Auswirkungen von Straßengütertransporten sowie die Operationalisierung der Belastungen Flächeninanspruchnahme und Zerschneidung die Verknüpfung menschlicher Aktivitäten mit dem weiten Thema Biodiversität in einem Bereich zu konkretisieren. Abgestimmt auf die Verwendung in Unternehmern sollen sie diesen einen nachhaltigeren Umgang mit den begrenzten ökologischen Ressourcen der Erde ermöglichen. Drei Handlungsempfehlungen, die in Einklang mit den hier vorgestellten Indikatoren stehen, sind erstens eine Reduktion und zweitens eine Bündelung von Verkehr auf durchlässigen Straßen sowie drittens die Erkenntnis, dass eine erhebliche Mehrnutzung umweltfreundlicherer Verkehrsmodi sowohl weiter steigende Transportvolumina als auch bestehende Belastungen für Mensch und Natur nicht unproblematisch macht.

\section{Literaturverzeichnis}

AULIG, G., BACHFISCHER, R., DAVID, J. \& KIEMSTEDT, H. (1978): Wissenschaftliches Gutachten zu ökologischen Planungsgrundlagen im Verdichtungsraum Nürnberg-Fürth-Erlangen-Schwabach. München (Lehrstuhl für Raumforschung, Raumordnung, Landesplanung TU München)

EUROPÄISCHE UMWELTAGENTUR (2003): Europe's environment: the third assessment. - Kopenhagen (EEA)

FAHRIG, L. \& RYTWINSKI, T. (2009): Effects of roads on animal abundance: an empirical review and synthesis. - Ecology and Society, 21: 1-20

Forman, R.T., Sperling, D., Bissonette, J.A., Clevenger, A.P., Cutshall, C.D., Dale, V.D., Fahrig, L., France, R., Goldman, C.R., HeAnue, K., Jones, J.A., Swanson, F.J., TurRentine, T. \& WINTER, T.C. (2003): Road Ecology: Science and Solutions. - Washington, D.C. (Island Press)

GuINEE, J.B. (Ed.) (2002): Handbook on Life Cycle Assessment. - Dordrecht (Kluwer)

Hänel, K., Reck, H., BötTcher, M., West, M., Huckauf, A., Jessberger, J., AndRe, R., HerRmann, M., Klar, N., Walz, U., StRatmann, L. \& Schumacher, J. (2010): Prioritäten zur Wiedervernetzung von Lebensraumkorridoren im überregionalen Straßennetz - Netzwerk für Wald bewohnende, große Säugetiere.

http://bfn.de/fileadmin/MDB/documents/themen/eingriffsregelung/2012_Prioritaeten_Gro\%DFsae uger_Layout_A0_300dpi.pdf, Zugriff am 08.08.2012 um 13:42 Uhr

Herrmann, M., ENSSLE, J., SÜSSER, M. \& KRÜGER, J.-A. (2007): Der NABU-Bundeswildwegeplan. - Meckenheim (Wahrlich)

Hubo, C., Jumpertz, E., Krott, M., Nockemann, L., Steinmann, A. \& BräUer, I. (2007): Grundlagen für die Entwicklung einer nationalen Strategie gegen invasive gebietsfremde Arten. - Bonn (BMU)

IPCC (2007): Synthesis Report. - Valencia (IPCC)

18 STENGEL 2011, S. 14 
JAEGER, J.A., GRAU, S. \& HABER, W. (2005): Einführung: Landschaftszerschneidung und die Folgen. - Gaia, 14: 98-100

LAtouche, S. (2009): Farewell to growth. - Cambridge (Polity Press)

Meadows, D.H., Meadows, D.L. \& Randers, J. (2004): The Limits to Growth: The 30-Year Update. - Vermont (Chelsea Green Pub Co)

MillenNiUm Ecosystem AsSeSSMENT (2005): Ecosystems and Human Well-being: Synthesis. Washington, D.C. (Island Press)

OECD (1993): OECD Core Set of Indicators for Environmental Performance Reviews: A synthesis report by the Group on the State of the Environment. - Paris (OECD)

RECK, H., HÄNEL, K., BÖTTCHER, M., TILlMANN, J. \& WinTER, A. (2005): Lebensraumkorridore für Mensch und Natur. - Münster (Landwirtschaftsverlag)

ROEDENBECK, I.A. \& JAEGER, J.A. (2006): Auf dem Weg zu straßenökologischer Forschung auf Landschaftsebene. Themenheft ,Straßenökologie‘.- Naturschutz und Landschaftsplanung, 38: 297299

ULRICH, P. (1991): Ökologische Unternehmungspolitik im Spannungsfeld von Ethik und Erfolg. St. Gallen (Institut für Wirtschaftsethik an der Hochschule St. Gallen)

SEIDL, I. \& ZAHRnT, A. (2010): Postwachstumsgesellschaft. Konzepte für die Zukunft. - Marburg (Metropolis)

StEngel, O. (2011): Suffizienz - Die Konsumgesellschaft in der ökologischen Krise. - München (oekom)

Jan Friedrich

Prof. Dr. Jutta Geldermann

Professur für Produktion und Logistik

Platz der Göttinger Sieben 3

37075 Göttingen

E-Mail: jan.friedrich@wiwi.uni-goettingen.de 



\section{ARTICLE II}

Friedrich, J., and J. Geldermann. 2013b. Current practices of mitigating the ecologically harmful effects of roads - An assessment. In Societal dimensions of the conser-vation and utilization of biological diversity, eds. J. Friedrich, A. Halsband and L. Minkmar, pp. 341-358. Goettingen: Goettingen University Press.

Printed with permission for non-commercial use. 



\title{
Current practices of mitigating the ecologically harmful effects of roads - An assessment
}

\author{
Jan Friedrich and Jutta Geldermann
}

\begin{abstract}
Roads and vehicles have been established as largely indispensable facilitators of people's lives. They allow for accessibility and facilitate trade. Yet, the spatial function of the landscape is not limited to movements. Other demands include agricultural production, resource extraction from forestry or mining, human recreation, functioning ecosystems and the demands of other life forms, e.g. flora and fauna. Today's intensity levels of use of a dense road grid threaten various animal species. Motivated by human interests in utility and also by ethical values, humanity decided to try to suspend its adverse effects on biological diversity at large. In this article the state of the art and the trends of the effects of land use, material emissions, noise and light are analyzed. The central focus however resides on the mitigation of the currently most discussed and potentially severest effect, which is landscape fragmentation. The two major approaches of resolution currently applied and debated in science and politics are a corridor- and an area-centered strategy. Although both courses of action shouldn't be rivaling as they complement each other and as such are both indispensable, the discussion of their merits and pitfalls, which is at the center of this text, concludes on an ecological superiority of area concepts. Thus, this strategy of defragmentation should be prioritized as far as spatially conflicting human interests permit.
\end{abstract}




\section{Introduction}

Movement and speed, dominantly actualized by the means of motorized transportation vehicles, are frequently effected activities in people's lives and at large are positively valued in industrialized countries. Today, many people commute to work, they drive to shops to exchange their salaries for goods, and they use cars to carry out relational and recreational activities. Specialization in production and the spreading of the concept of paid labor instead of ownership over the output, low costs of transportation due to subsidies and due to the acceptance of external effects, as well as the opening of global markets have drastically increased the mobility-intensity of trade and of individual mobility patterns. A degree has been reached where high spatial and high energy demands of fast movements increasingly conflict with human interests and with the needs and health of many other life forms. Noise and emissions for instance have harmful effects also on animals and plants. Mobility thereby is one driver that contributes to the threat and loss of biological diversity.

The German federal government addresses this problem in formulating the following central goals for mobility under the National Strategy on Biological Diversity: "Impairments caused by traffic, for instance as a result of pollutants, noise and light, will be continuously reduced. New land transport routes (primarily road, waterways and rail) indicate adequate levels of ecological passability (e.g. fish ladders or wildlife crossings). By 2020, as a general rule, the existing transport routes will no longer cause any significant impairments to the system of interlinked biotopes. The current proportion of undissected, low-traffic areas of $\geq 100 \mathrm{~km}^{2}$ will be retained."1 In discussing land use, material emissions, noise, and light, this text analyzes the situation of four of the most relevant impairments when dealing with the ecologically adverse effects of roads and traffic. ${ }^{2}$ However, the focus of this text is on the effect of habitat fragmentation that is caused by linear transport infrastructure. After briefly referring to the history of human perception of the effect and after briefly explaining how individuals, species and ecosystems can be compromised by it, in the main part the two major approaches of resolution that are currently applied and discussed in science and in politics will be analyzed and assessed.

The reason for this emphasis is twofold: On the one hand, fragmentation is regarded as one of the most pressing but still underestimated environmental problems of the present. ${ }^{3}$ On the other hand, as ideas and strategies on how to tackle the problem develop and are implemented, it seems worthwhile to pause and to analyze their effectiveness. This seems relevant because even in the scientific community significant debate on the capabilities and on the interplay of the here entitled 'area-' and 'corridor-centered' approaches emerged at a recent global con-

\footnotetext{
${ }^{1}$ Federal Ministry for the Environment, Nature Conservation and Nuclear Safety 2007, p. 51f.

${ }^{2}$ For a more complete list of effects see Friedrich \& Geldermann 2013 and Forman \& Sperling 2011.

${ }^{3}$ Selva et al. 2011, p. $865 f$.
} 
ference on road ecology. ${ }^{4}$ To introduce to the topic and the discussion depicted in this text, the history of road ecology research is briefly presented.

Consciousness about the environmentally harmful effects of vehicles and roads dates back to 1925, when Stoner during a 632 mile car trip in the USA counted 225 animals that had died from collisions with vehicles. ${ }^{5}$ It then took until the $1970 \mathrm{~s}$ and -80 s when notable research mainly from Mader and Ellenberg marked the next regional milestone in the natural sciences dominated field. ${ }^{6}$ At an international and particularly at a North American level, the first comprehensive work concerning the entire variety of possible effects was entitled "Road Ecology - Science and Solutions". It was written by Forman et al. and was published in 2003. Since this date research has increased significantly, potentially because risen mobility usage has rendered the problem more evident, and additionally because scientific findings and political decisions to tackle the human induced rapid loss of biological diversity required also looking at the impacts of road use in more detail. In order to bring together scientists, infrastructure planners and politicians of all levels two interdisciplinary conferences, the ICOET- International Conference on Ecology and Transportation in North America and the IENE Conference in Europe, were established. The goal of road ecology research is to systematically identify and to assess the effects of roads and vehicles on the non-human environment and to then suggest solutions which shall help to avoid or to successfully mitigate harmful effects. In line with this objective, this text evaluates whether current efforts are apt to fulfill the goals of the national strategy or whether different targets and more ambitious policy action are necessary.

\subsection{Landscape Fragmentation}

The dissection of natural areas is called landscape or habitat fragmentation. ${ }^{7}$ It occurs predominantly because of the construction and use of linear transport infrastructure. It further results from areas intensively used by humans, like settle-

\footnotetext{
${ }^{4}$ This refers to the IENE International Conference, held in Potsdam, Germany, in October 2012. The Infra Eco Network Europe (IENE) is an expert network of authorities, planners and researchers involved in the phenomenon of habitat fragmentation caused by the construction and use of transport infrastructure, especially roads, railways and waterways.

${ }^{5}$ Cp. Stoner 1925.

${ }^{6}$ Roedenbeck \& Jaeger 2006, p. 297.

7 The term "landscape" is not entirely anthropogenic. Besides its culturally driven meaning of an area influenced by humans it is also used to distinguish between areas with differing species compositions or ecosystems. The word "habitat" sets a clear significance to the understanding that animal and plant species live among us and that they have their respective needs for soil and ecosystem characteristics. Hence, at first sight the term habitat fragmentation seems to be more suitable to be used for the issue at hand. However, in continuation the term landscape fragmentation shall be used because it denotes a more holistic understanding than does the species-associated word habitat. It is viewed that a species focus would mislead, because fragmentation is a broader problem. While it has to consider majorly affected species in Germany, only a very small amount of wilderness exists and most habitats are humanly impacted. Therefore, an approach dealing with these areas should openly consider the needs and preferences of both human and non-human nature.
} 
ments, industry, or agricultural area with few natural components. The manifold values of non-sealed surface area (e.g. soil fertility, water drainage, processing of wastes and water) together with still high rates of uptake of such area lead to an in theory significant valuation of natural surfaces. While the first specific goals to reduce land uptake were formulated in 1998, the impacts of disconnecting landscapes and natural processes were less apparent and they were barely recognized until much later. ${ }^{8}$ Two publications by Lassen in 1979 and 1987 deal with the phenomenon of large undissected areas becoming scarce. Reasoning why this development was undesirable, Lassen argues that such areas were important for people to rest and to recover. ${ }^{9}$ Hence problem identification had a clear anthropogenic emphasis. For most countries, and particularly for those with road networks as dense as Germany's, this has changed today. Most books and articles that deal with the effects of roads in specific or with the degradation of ecosystems, with the loss of biodiversity, or with another topic that is related to the imbalance of Western societies' lifestyles and the demands and condition of the environment, usually mention fragmentation as one of the major human induced ecological threats. ${ }^{10} \mathrm{By}$ today, in many parts of the world, particularly in countries with dense road grids, but also in places where the effects of dirt roads for forestry were concerned, studies on many species and their perturbation by roads were conducted. ${ }^{11}$ Besides the impact of habitat loss and degradation of adjunct area, mortality in the road and the road representing a barrier to movement or having a dissecting impact were identified as significant negative impacts of roads. ${ }^{12}$ The effects are visualized in the following graphic by Jaarsma and Willems.

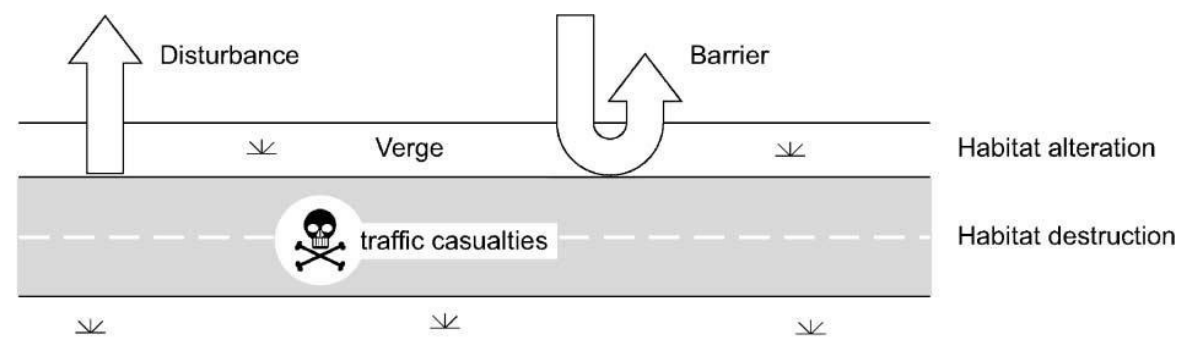

Figure 1: The potential impacts of roads and traffic on flora and fauna; adapted from Jaarsma \& Willems 2002

Particularly affected are slow-moving species (e.g. amphibians, hedgehogs or vipers), species with low dispersal abilities (e.g. flightless beetles), noise sensitive species (e.g. birds, toads, bats or moose), and species with larger habitat demands

\footnotetext{
${ }^{8}$ Federal Ministry for the Environment, Nature Conservation and Nuclear Safety 1998, S. 2.

${ }^{9}$ Cp. Lassen 1979, p. 333f; Lassen 1987, p. 534.

${ }^{10}$ Federal Environmental Agency 2010, p. 9ff; Lindenmayer 2006, p. 11; Schupp 2005, p. $101 \mathrm{ff}$.

11 Selva et al. 2011, p. 866; cp. Grez et al. 2006 (in references see 'Lindenmayer 2006').

12 Own interpretation based on Jaeger et al. 2005, p. 330f.
} 
that have more extensive movement ranges (e.g. wild cat, deer, lynx). ${ }^{13}$ Due to a time lag between the occurrence of the impact and the appearance of the full ecological consequences in the landscape, research and even more so societal awareness of the problem are handicapped. Although only few studies exist that have proven a reduced variability and reduced vitality in animals and plants due to fragmentation, the genetic disadvantageous effect is rarely questioned. ${ }^{14}$ In addition, also hydrologic flows are frequently affected by fragmentation. ${ }^{15}$ Overall, scientific research has moved beyond the point of problem identification. Studies and debates now center on the most appropriate ways to avoid and to mitigate the adverse effects of the dissection of populations, of the barrier effect and of animal mortality in roads. ${ }^{16}$ It is necessary to bear in mind all three effects simultaneously, because they are highly interrelated. Mitigation measures affect species differently and trigger differing responses. For instance, fencing may lower the risk of road mortality for some animals but it is likely to increase the barrier function for others unless connectivity-enabling efforts are carried out at the same time. The text will now move on to introduce and to discuss the two currently most popular strategies of defragmentation.

\section{The Two Major Approaches of Defragmentation}

\subsection{The corridor-oriented approach}

The identification of movement corridors of different habitat-type species in the first step, and the intent to establish connectivity along these corridors through the construction of wildlife crossings in a second step, in the following is referred to as a corridor-oriented strategy of tackling fragmentation. ${ }^{17}$ While culverts serving wetland species, and viaducts serving also dry- and forest-habitat species, in the past had been built mostly for geological and constructional reasons, today overand underpasses are predominantly built with the aim to serve amphibians and mammals. Since casualties in the road or on roadsides were most obvious for larger mammals like rabbits or deer, and since it was found that movements and behavior of larger mammals have a particularly positive function on the diversity of ecosystems, the approach of building larger overpasses instead of using fences and road signs is lately followed more intensively. ${ }^{18,19}$ The analysis of the corridor-

\footnotetext{
${ }^{13}$ Hels \& Buchwald 2001, p. 337; Keller et al. 2004, p. 2993; cp. Eigenbrod et al. 2009; Shanley \& Pyare 2011, p. 13.

14 Cp. Keller \& Largiader 2003; Fischer \& Matthies 1998.

15 Forman et al. 2003, p. 179.

16 van der Grift et al. 2013, p. 425.

17 Different habitat types include wetlands, such as bogs and fens, dry habitats, such as dunes, sandy or mountainous areas, some grasslands or desert-type of landscapes, and various forest-type habitats.

18 Reck et al. 2009, S. 4.
} 
oriented approach in this text refers to these larger structures (see an example in figure 2) and to larger mammals, but it is valid for other types of species as well.

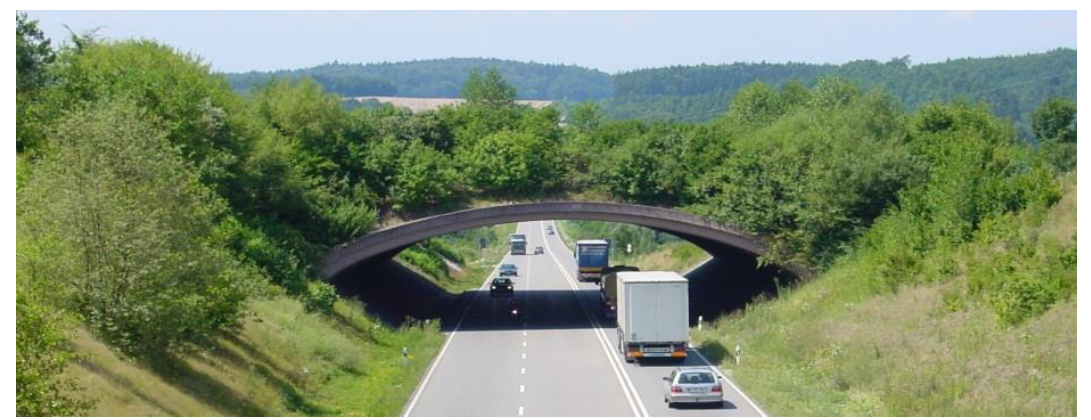

Figure 2: Image of a wildlife overpass; picture by Martin Strein, FVA Freiburg

In 2012, Germany had about 30 green bridges, 18 more were commissioned under the Economic Stimulus Package No. II, and about 50 bridges are to be built until $2020 .{ }^{20}$ The cost of an overpass usually is 3 to 5 million euros. Although bridges in place today seem to work for larger mammals, a variety of important aspects needs to be considered during construction, "operation", and maintenance of these structures. The following seven criteria were found to be imperative: 1) the correct positioning of a structure should be derived from analyzing road mortality rates, from the importance of connecting certain habitats, or from the demands of the species concerned 2) the necessity of complementary structures like fences has to be investigated, 3) the minimum width should be 50 meters, 4) to attract differing species, bridges must have a cover of diverse vegetation, 5) a functioning hinterland connection, which connects the bridge and its immediate surroundings to the wider landscape, has to be established, 6) tests on the achievement of preset goals concerning a species, its populations or an entire ecosystems have to be conducted; proves of the use of the bridge from taping animals using the bridge on film can only be a first step because while use is essential for effectiveness it does not imply that effectiveness - that is the fulfillment of the concrete goals - is attained, and 7) donor populations should be strengthened in order to motivate individuals or small groups to move further away from their current core habitats. ${ }^{21}$

Despite the above findings, the ideal composition of large crossing structures and of their near surroundings is still insufficiently known. ${ }^{22}$ The nature of the

\footnotetext{
${ }^{19}$ Larger mammals have been designated to have a so called vector-function for the diversity of local and regional ecosystems for two reasons: Firstly, they cover large distances both in their daily home range movements and in their seasonal dispersal ranges. Since they carry insects or spores in their fur and under their hooves they spread individuals across large areas. Secondly, they often destroy plants and bushes when they move and feed and therewith create more structurally diverse local ecosystems. ${ }^{20}$ Federal Ministry for the Environment, Nature Conservation and Nuclear Safety 2010, p. 40.

${ }^{21}$ Reck et al. 2011, p. 9; Iuell et al. 2003, p. 13ff.

22 van der Grift et al. 2013, p. 427.
} 
hinterland connectivity, the interplay of complementary components, the role of donor habitats, and particularly representative before- and after-investigations at the population level, are some of the topics that require further investigation. Despite recorded overpass uses by animals, so far no evidence on their effectiveness in mitigating genetic depletion at the population level exists. ${ }^{23}$ While the evidence of their casualties as well as the assumed vector-function of larger forest species endorses the importance of large bridge-type crossing structures, in total a large variety of structures exists. These include underpass tunnels, viaducts, amphibian tunnels, fish ladders, rope bridges, glider poles, tunnels and culverts, and even greened roofs in industrial or urban areas. In spite of the strength of centrally tackling the usually most severe threat of fragmented habitats and ecosystems, that is reduced genetic variability, the following three reasons show why crossing structures alone cannot suspend all effects sufficiently:

1) even a variety of differing and functioning types of bridges or structures cannot serve all species and their respective dissimilar needs adequately,

2) the number of identified locations where wildlife was found to be severely affected is far greater than there is money available to build the necessary structures (900 identified locations vs. 50 to 80 structures planned until 2020) $)^{24}$, and

3) the identification of corridors yields the danger of a dominance of mitigating approaches over strategies of avoidance.

Particularly the last point leaves room for debate as it relates to the broader question of the overall degree of sparing nature from negative impacts of roads and traffic. Before referring to this debate in the discussions section, next, the areaoriented approach is introduced and its benefits and drawbacks are explained.

\subsection{The area-oriented approach}

Despite a long lasting negative trend for the share of large lowly-disturbed areas in Germany, their importance for functioning and stable ecosystems is explicitly acknowledged in Germany's National Strategy on Biological Diversity. ${ }^{25}$ However, even the 2010-target not to have these areas diminish further than the last known value of 25.4 per cent, was already missed by far in $2011 .{ }^{26}$ One possible definition of such areas and the one used in Germany is as follows: areas need to have a minimum size of $100 \mathrm{~km}^{2}$, and they cannot be dissected by a road with a traffic intensity greater 1,000 vehicles per day, by railways with two or more tracks or with any

\footnotetext{
${ }^{23}$ Corlatti et al. 2009, p. 548; van der Grift et al. 2013, p. 425.

${ }^{24}$ Herrmann et al. 2007, p. 26.

25 Originating from different countries and introduced by different researchers, many terms exist to describe areas with low disturbances by humans. The most used expressions are roadless area, (undissected) low-traffic area, or traffic calming rural area. The main variable is traffic, but also settlements and industry can be disturbing or fragmenting elements. For details the specific fragmentation geometry has to be looked at. In this text the term lowly-disturbed area will be used most.

${ }^{26}$ Federal Ministry for the Environment, Conservation and Nuclear Safety 2010, p. 41 \& 2007, p. 41.
} 
electrified track, or by a waterway greater than category III. In addition, there cannot be tunnels larger than one kilometer, water bodies which are larger than half of the total area, or larger settlements or airports within the area.. ${ }^{27}$ Large lowlydisturbed ecological areas are of cardinal ecological importance because they hold conducive premises for biological diversity and for ecosystem stability. ${ }^{28}$ With regard to biodiversity, large lowly-disturbed ecological areas have several advantages: they support the preservation of native biodiversity and their genetic resources, they can function as barriers against invasive species, they ensure habitat for the viability also of larger area demanding populations, and they maintain ecosystem connectivity as they often function as migration corridors and stopovers. Moreover, ecosystem stability is supported because larger natural areas have a high buffering capacity. This translates into augmented resilience to external pressures, like pests, climatic changes, or other catastrophic events, like fires, landslides, or floods. ${ }^{29}$ Another quality of larger areas that are without significant human influences, particularly at this point in human history, is their ability to withdraw carbon from the air and hence to decrease the atmospheric greenhouse gas effect..$^{30}$

The area-approach has two major disadvantages: firstly, large lowly-disturbed areas are not available for intensive human use. Humans have many needs. Among the most basic ones are the urges to protect and to sustain themselves by building shelter, eating food, and by engaging in relationships. Some of these demands require space and soil. While it seems possible to live in ways that interfere with the interests of other life forms in a respecting and sustainable manner, for the majority of people in capitalist societies the perception of a fulfilling lifestyle today is a personal utility-maximizing consumptive one, rather than a convivial sufficient one. This creates spatial conflict between large demands for resources and space and between the idea of having lowly-disturbed large natural areas. This leads to the problem, which only to a much lower degree arises from sufficiency-oriented lifestyles and a higher appreciation of other life forms, that decisions and choices on the designation of lowly-disturbed areas need to be made. Which percentage of land mass or waters should be exempted from human use? Are there preferred habitat types? What is the definition of a lowly-disturbed area, and which human activities are allowed? ${ }^{31}$ It is evident that an area-oriented approach is of high ecological quality and that it at the same time requires notable changes in values and behavior in order to be exercised at large scale.

\footnotetext{
27 Schupp 2005, p. 105.

28 Selva et al. 2011, p. 867.

${ }^{29}$ For a complete list of human benefits from ecosystems see Haines-Young \& Potschin 2010, p. $11 \mathrm{ff}$.

30 Selva et al. 2011, p. 867.

${ }^{31}$ Most important to be considered are the five topics of mobility, settlements, commercial and agricultural use, and recreation. What is the fragmentation geometry concerning mobility infrastructure? How large can villages and cities be within such areas? Can there be mining activity and what percentage or intensity of agricultural activity or forestry is permissible? What are the terms of recreational use? Are there inaccessible wilderness areas?
} 


\subsection{Discussion}

While a corridor-oriented strategy currently seems to be prioritized in science and in practice, experts concede that efforts of avoidance like maintaining and reestablishing large lowly-disturbed areas, offer the greatest ecological benefits. ${ }^{32}$ The two major drawbacks of large crossing structures are that they only are assumed to successfully tackle the problem of fragmentation for a certain number of species in certain places, and that the impression of a fence and a crossing structure, and no further visible animal casualties in the road can lead to the false perception of all adverse effects of traffic being overcome. These two problems are the strongholds of an area-oriented approach. It provides connectivity for most species in most places and also reduces other effects like substance emissions or noise in great parts of the landscape. This applies only for within traffic-calmed areas. Thus, the need to connect such areas to one another remains, and corridors and crossing structures are indispensable for this matter. Yet, at a greater landscape level lowtraffic areas are vastly superior.

In addition, there is an ethical dimension to the comparison of the two strategies. While building crossing structures in prioritized sites is a mitigating, end-ofpipe attempt, which indicates a rather minimalistic valuation of biodiversity and of non-human life, self-imposing significant restrictions at a landscape level equal an approach of avoidance that tells of high respect and interest in the conservation of biodiversity. Concerning the related concept of sustainability, Becker argues that humanity struggles to define its meaning "because it is about a fundamental and demanding philosophical issue of human existence: the issue of the self-identity and existence of the human being as a fundamentally dependent and relational being." According to Becker, the issue is so demanding because the desired status of three fundamental relationships and dependencies has to be answered: The relation with other contemporaries, with nature, and with past and future generations. ${ }^{33}$ The issue of how to deal with the impacts of roads and traffic is related to these demanding questions.

From the analysis of the two strategies of defragmentation, the first conclusion is that both approaches have their merits. The stronghold of lowly-disturbed areas is ecological quality. Installing crossing structures has the advantage that despite their high cost, the overall cost to society might be perceived as acceptable because no changes in economic organization and lifestyles are necessary since the level of mobility intensity can persist. Secondly, even assuming changes in behavior and after exercising the frequently suggested bundling of traffic, fewer but yet a significant number of fragmenting conflicts will exist and artificial structures will be indispensable in these cases. ${ }^{34}$ The second conclusion is that the degree, to which the

\footnotetext{
32 Iuell et al. 2003, p. 32; EEA 2011, p. 67; Benítez-López et al. 2010, p. 1314; Selva et al. 2011, p.

874; Forman \& Sperling 2011, p. 16

33 Becker 2010, p. 3.

${ }^{34}$ Cp. Friedrich \& Geldermann 2013; Charry \& Jones 2009, p. 168; Jaeger et al. 2006, p. 151.
} 
problem of fragmentation should be answered by installing crossing structures, should be carefully chosen. It can be argued to be morally wrong, or said to be rationally naïve to profusely trust in an unconfirmed, species-centered, and rather minimalistic approach, when trying to maintain biological diversity and ecosystem functionality. Biodiversity and ecosystems have been developing for much longer than human existence, in fact they are the source of the human species, and humans also today fragilely depend upon them.

\section{Further impairments of roads and traffic on nature}

In this last section, the current conditions of further ecologically harmful effects of roads and traffic are referred to. The first aspect to turn to is land use. ${ }^{35}$ In 2010, 4.39 per cent of Germany's total land mass was used for road bound mobility and the absolute area value has increased constantly since 1992.36 The goal to reduce total land use to 30 hectares per day by 2020 was first formulated by the former environmental minister Angela Merkel in 1998. ${ }^{37}$ In spite of an overall positive trend, the average rate of reduction of about 3 hectares per year is too low to reach the target by $2020 . .^{38}$ Nevertheless, this target was confirmed and enlarged in 2007, as land use shall be driven close to zero after $2020 .{ }^{39}$ Reasons that allow to still being confident of achieving the objectives are that policy analyses and efforts seem to be increasing and that the mindset within the respective ministries is clear: "The realization of the national aim to reduce land consumption to 30 hectares by 2020 is no self-priming pump, nor is it always a win-win situation. It therefore requires intensive governance". ${ }^{40}$ Besides, the percentage of land surface affected by roads is much higher than the 4.39 per cent referred to above, because ecologically relevant effects extend beyond the physical location of the road. The most intuitive and - apart from greenhouse gas emissions - most severe impact is noise. In addition, further substance emissions from vehicles and from the road, as well as changes in local climate and in hydrologic systems occur. The area over which these ecological impacts extend is called 'road-effect zone. ${ }^{41}$ Its width varies for differing species and it strongly correlates with traffic volume. ${ }^{42}$ Its shape is often

\footnotetext{
${ }^{35}$ Land use or land consumption is defined as the increase in the amount of land used for human settlements, industry, recreation, and transport infrastructure. The characteristic that differentiates land use from other categories like forests or agriculture is that high proportions of land are sealed.

${ }^{36}$ Federal Statistical Office 2011, p.23f; calculations extended by the authors.

${ }^{37}$ Federal Ministry for the Environment, Nature Conservation and Nuclear Safety 1998, S. 2.

38 The four year mean value for the period 1995 to 1998 was about 124 hectares per day and the value for the years 2007 to 2010 was 87 hectares per day (Federal Statistical Office 2011, p. 18.).

${ }^{39}$ Federal Ministry for the Environment, Nature Conservation and Nuclear Safety 2007, p. 51.

${ }^{40}$ Cp. Henger et al. 2010; Federal Ministry of Transport, Building \& Urban Development 2011, p. 69.

${ }^{41}$ Forman \& Deblinger 2000, p. 37.

${ }^{42}$ E.g. Salamanders were found to be affected by forest roads for a distance of 35 meters (Semlitsch et al. 2007), breeding birds within the first 200 meters (Reijnen et al. 1995), tortoise for up to 400 meters (Boarman \& Sazaki 2006), and anuran species for up to 1,000 meters (Eigenbrod et al. 2009).
} 
asymmetric because of the strong influence of local topography. Biglin \& DupignyGiroux found the majority of impacts to occur within the first 100 meters, Forman and Deblinger estimate an average width of approximately 600 meters, and impacts can extend for several kilometers for noise sensitive species. ${ }^{43}$ Considering this, Forman estimated the 1 per cent of surface covered by roads to essentially affect 20 per cent of total U.S. land mass. ${ }^{44}$ Starting at 4.39 per cent of the surface being taken up by roads in Germany, the percentage for the area affected by road-use can be estimated to be very high. This also argues in favor of systematically maintaining and re-establishing large lowly-disturbed areas, particularly in countries with dense road grids as in the case of Germany. All in all, the loss of the functions of natural soil, the loss of fertile arable area, and the loss of ecologically intact area, including its biodiversity, do strongly argue to trying to reach the before mentioned objectives to reduce and to halt land use, and to also reduce the extending effects. 45

With regard to emissions, it seems helpful to distinguish between material and immaterial discharges. Substance emissions were found to potentially produce genetic defects in small mammals, to repress germination in plants, and to alter the competition and composition of vegetation for up to 200 meters. ${ }^{46}$ The most relevant material emissions are nitrogen oxide, particulate matter, ozone, carbon monoxide, carbon dioxide, sulfur dioxide, non-methane hydrocarbons or volatile organic compounds, and also rubber, dust and litter. The two major immaterial emissions are light and noise. As for material discharges, considerable reductions have been achieved for most substances. Yet, for nitrogen oxide, particulate matter and ozone maximum permissible values were still exceeded repeatedly in $2011 .{ }^{47} \mathrm{Com}-$ paring emission levels from 1995 and 2010, nitrogen oxide emissions could be cut back by 65 per cent, particulate matter by about 70 per cent, and sulfur oxide by over 98 per cent. ${ }^{48}$ Alone improvements of climate change provoking carbon dioxide for cars of about 10 per cent and of 28.3 per cent for lorries reach unsatisfying levels. All in all, further reductions particularly for nitrogen oxide, particulate matter, and chiefly for carbon dioxide need to be achieved in the future.

With regard to immaterial emissions like heat, vibration, sound, and light, the latter two seem to be the most harmful ones. Sound is a mechanical wave and its loudness is measured in decibels $(\mathrm{dB}[\mathrm{A}])$. The classification ' $\mathrm{A}$ ' refers to the frequency dependent responsiveness of the human sense of hearing. When sound is perceived as a strain, it is referred to as noise. Animal species show all three possible general responses to human-caused noise: attraction, tolerance, and aversion. Similarly as for humans disturbance is the most dominant and most problematic

\footnotetext{
43 Biglin \& Dupigny 2006, p. 13; Forman \& Deblinger 2000, p. 36; Jordaan et al. 2009, p. 3.

${ }^{44}$ Forman 2000, p. 34.

${ }^{45}$ Frequent suggestions include to concentrate traffic and to downgrade or close rural roads, as well as to use soil berms and to reduce vehicle noise (Forman 2000, p. 35; Charry \& Jones 2009, p. 168).

${ }^{46}$ Rassmus et al. 2002, p. $109 f_{s}$ Forman et al. 2003, p. 212 f.

${ }^{47}$ Federal Environmental Agency 2012a, p. 4.

${ }^{48}$ Federal Environmental Agency 2012b, p. 12.
} 
perception. It can show as (1) annoyance, (2) hearing loss, (3) 'speech' and sleep interference, (4) stress-related illness, (5) humans perceived as predators, (6) population changes, (7) genetic change over generations, and in the form of (8) numerous other behavioral responses. ${ }^{49}$ Despite having recognized the disturbances of sound on humans and animals, few recordings have been conducted and so few and scattered data is available. Existing recordings until today are limited to analyze the extent of sound that disturbs people. This means that even in a scenario of successful mitigation the development of noise occurring at the landscape level is not displayed. Overall, it is difficult to assess, whether the impact of noise on plant and animal species has increased in magnitude or has been abated in the past. ${ }^{50}$ However, considering the development of technological and behavioral criteria, analysis of the few existing data and also expert opinion suggest that the effect has increased, both in the extent emitted at the sources as well as in the level of disturbance perceived. ${ }^{51}$ One indication is that despite reductions being achieved through advances in vehicle technology, which for instance show in lower sound limits for the registration of new cars, the quantitative increases in traffic volume outstrip the technical improvements. Furthermore, also behavioral changes outweigh technological diminutions. The dominant source of noise switches from stemming from the engine to stemming from the friction of the wheel at a speed of about $50 \mathrm{~km} / \mathrm{h}$. Advances towards quieter engines hence have been outbalanced by peoples' faster driving behavior and the respective necessary usage of wider tires to maintain road grip for safety reasons. For a more precise assessment and for more effective approaches of resolution, increased monitoring of the quantity of noise emissions, also occurring at the landscape level, are necessary. Since 2007 the German Federal Environmental Agency is collecting recordings for sound from the federal states and after publishing the first nationally compiled data for the year 2010, the data for 2012 will follow in 2013.52

Lighting can impact ecosystems because together with temperature and humidity, light is the most important zeitgeber for the biological clock of many plant and animal species. It largely governs their daily and seasonal temporal behavior. One example would be that animals, and also humans, need a sufficient quantum of darkness for the daily regeneration of their hormonal and immune systems. ${ }^{53}$ In addition to this temporal dimension, there exists a second problematic dimension, which is spatial. This means that light can attract and endanger animals, as in the case of nocturnal insects like moths, or that it can disturb animals, for instance in diminishing their habitats or in troubling their orientation. ${ }^{54}$ Particularly affected

\footnotetext{
${ }^{49}$ Forman et al. 2003, p. 273.

${ }^{50}$ German Advisory Council on the Environment 1999, p. 158; Forman et al. 2003, p. 270; cp. Federal Agency for Nature Conservation 2001.

${ }^{51}$ Cp. Federal Agency for Nature Conservation 2009; cp. Hintzsche 2012.

${ }^{52}$ Federal Environmental Agency 2012b, p. 50.

53 Eisenbeis 2001, p. 98 (in Federal Agency for Nature Conservation 2001).

${ }^{54}$ Schmiedel 2001, p. $19 \mathrm{f}$ (in Federal Agency for Nature Conservation 2001).
} 
species are insects, amphibians, reptiles, and birds, and also plants. While data availability is poor with regard to sound, the situation is worse for lighting. Estimations for the global level say that planet earth is getting brighter night by night..$^{55}$ There are also studies on individual municipalities, for instance for Berlin. The city's sky glow is one of many subjects in Germany's currently most extensive research project called 'Loss of the Night', which is supported by the Federal Ministry of Education and Research. ${ }^{66}$ One number concerning light emissions is known from an expert conference on light pollution and biodiversity, held in Tutzing, Germany, in 2011. Total flux, reported in $\mathrm{lm}$ for Latin 'lumen', is the measure for the entire visible brightness emitted from a source of light. The measure complies with the responsivity of the human eye. For Germany, from the year 1981 to 2011 it is assumed to have risen by 120 per cent and that is accounting for all shifts in light bulb technology and changed installation quantities. ${ }^{57}$ The particular share of roads and traffic in this increase was not identified. However, an ongoing enlargement of the road network and an increase in traffic volumes make an opposing development for light emissions stemming from mobility unlikely. In spite of having acknowledged the dangers of artificial light for flora, fauna and humans, similar to the situation for traffic noise, technological advances in lighting in general have been outnumbered by increased lighting use. In conclusion, it seems that the trend for both noise and light emissions and the felt disturbances is still increasing.

\section{Summary and future outlook}

Scientists have been successful in explaining to political leaders the severity of the ecological effect of fragmentation, which is predominantly caused by transport infrastructure. Now, a new debate within the scientific field as well as in the political landscape has arisen over the question of the best mix of measures to resolve this issue. The word 'best' indicates that a fundamental, even philosophical dimension plays a role in this debate. While the goal to maintain biological diversity is commonly agreed upon, the degree of ambition on how much variety or quantity of diversity is needed or wanted is unclear. Also the attitude towards environmental risks, which is vaguely established by the precautionary principle, of which current national and global environmental policies and people's actions far from resemble, is still being discussed in contemporary societies. This dilemma also shows in Germany's national strategy, where it says that 'adequate' levels of ecological passability need to be achieved. At the policy level one facet of this is the weighting of the approaches of avoidance, mitigation, and compensation. In this light, the text at hand discussed the advantages and pitfalls of the two currently most popular measures when dealing with the ecologically adverse effects of fragmentation.

${ }^{55}$ Cp. Federal Agency for Nature Conservation 2009.

${ }^{56}$ See 'Loss of the Night', Leibniz Institute of Freshwater Ecology and Inland Fisheries, Berlin 2013.

${ }^{57}$ Isepy 2012. 
The approach of maintaining and re-establishing roadless or lowly-disturbed areas values diverse and functioning landscapes and ecosystems to a very high degree. In following a strategy of avoidance it sets great store by the precautionary principle. In a corridor-oriented approach conflict zones are prioritized and connectivity for wildlife is attempted to be re-established by the construction of crossing structures. It is a strategy of mitigation, which values higher the human demands on land and which deals with ecological complexity more optimistically. Both approaches have to be exercised; only their proportions have to be chosen carefully. The discussion of their advantages and weaknesses meant to contribute to this end.

The analysis of other impairments caused by traffic yielded that the goal of Germany's National Strategy to continuously reduce them is insufficiently reached. The positive trend for land use is too weak. In spite of significant improvements achieved in the past, a further reduction of material emissions, and here particularly carbon dioxide, remains necessary. While the disturbance of far-reaching impacts like noise and light is known, more measurements and research are needed to track their development and to apply effective strategies of avoidance and mitigation.

Looking into the future, research opportunities for people dealing with the abolishment of ecologically harmful effects of roads are numerous. Further robust studies on the functionality of crossing structures, especially concerning geneflow at a population level and ensuing ecosystem responses are needed. ${ }^{58}$ While for lowly-disturbed areas, more research on thresholds of traffic volumes, noise, and light is needed, the overall recommendation to concentrate traffic on primary roads and to downgrade or close roads in natural landscapes has been repeated for more than ten years. ${ }^{59}$ Moreover, despite valid criticism on the suitability of monetizing the value of non-human life forms, actions and policies with the objective to constitute the 'the user pays principle' have to be pursued. Similar to the rebound effect, increases in traffic volumes and speed have outweighed the individual unit reductions of noise and emissions. Therefore, road ecologists and mobility planners have to seek approaches of avoidance to a greater degree. To this end, environmentally friendlier modes of movement like biking or collective vehicle use have to be analyzed and supported. Despite significant opportunities existing in the field today, it shall be pointed out that the need to move is only partially of intrinsic nature and that many movements are a result of societal values, like speed, consumerism, individualism, or internationalism, and also of economic structure and paradigms. If desired, changes can occur in these spheres as well. From a philosophical perspective, more thoughts on the human relation to nature and the resulting implications for instance for the meaning of the precautionary principle in the context of roads seem due. Also, road ecologists should not limit their studies and expertise to roads because for instance regarding the effects of trains similar impacts and significant synergies can be expected to exist.

\footnotetext{
${ }^{58}$ Corlatti et al. 2009, p. 548; van der Grift et al. 2013, p. 444.

${ }^{59}$ Jaarsma \& Willems 2002, p. 168; Charry \& Jones 2009, p. 168; Forman \& Sperling 2011, p. 19.
} 


\section{References}

Becker, Ch.: Sustainability ethics. 2010, STS Program and Department of Philosophy, Pennsylvania State University.

Benítez-López, A.; Alkemade, R. and Verweij, P.A.: The impacts of roads and other infrastructure on mammal and bird populations: a meta-analysis. 2010, Biological Conservation 143, p. 1307-1316.

Biglin, K. and Dupigny-Giroux, L.-A.: Mapping the road-effect zone to assess impacts of proposed road segments. 2006, Journal of Conservation Planning 2, p. 1-16.

Boarman, W. I. and Sazaki, M.: A highway's road-effect zone for desert tortoises(Gopherus agassizii). 2006, Journal of Arid Environments 65, p. 94101.

Charry, B. and Jones, J.: Traffic volume as a primary road characteristic impacting wildlife: a tool for land use and transportation planning. In: Wagner, P. J.; Nelson, D. and Murray, E. (eds.): Proceedings of the international conference on ecology and transportation. 2009, Center for Transportation and the Environment, p. 159-172.

Corlatti, L.; Hackländer, K. and Frey-Roos, F.: Ability of wildlife overpasses to provide connectivity and prevent genetic isolation. 2009, Conservation Biology 23, p. 548-556.

EEA (European Environmental Agency): Landscape fragmentation in Europe Joint EEA-FOEN report. 2011, EEA.

Eigenbrod, F.; Hecnar, St. J. and Fahrig, L.: Quantifying the road-effect zone: threshold effects of a motorway on anuran populations in Ontario, Canada. 2009, Ecology and Society 14, p. 1-18.

Federal Agency for Nature Conservation: Auswirkungen von Fremdlicht auf die Fauna im Rahmen von Eingriffen in Natur und Landschaft. 2001, Schriftenreihe für Landschaftspflege und Naturschutz 67, p. 1-192.

Federal Agency for Nature Conservation: TV-Dokumentation: Die dunkle Seite des Lichts, Lichtverschmutzung - ein Problem für Pflanzen und Tiere. 2009, http://www.bfn.de/0401_pm.html?\&cHash=f6dd938e12\&tx_ttnews[backPid] =1\&tx_ttnews[tt_news] $=1272$. (accessed December 18 $\left.{ }^{\text {th }}, 2012\right)$.

Federal Environmental Agency: Maintaining biodiversity by protecting the environment. 2010, Federal Environmental Agency.

Federal Environmental Agency: Air quality 2011. 2012a, Federal Environmental Agency. 
Federal Environmental Agency: Daten zum Verkehr. Ausgabe 2012. 2012b, Federal Environmental Agency.

Federal Ministry for the Environment, Nature Conservation and Nuclear Safety:

Mit dem Entwurf eines umweltpolitischen Schwerpunktprogrammes neue

Etappe in der Umweltpolitik eingeleitet. 1998, BMU.

Federal Ministry for the Environment, Nature Conservation and Nuclear Safety:

National strategy on biological diversity. 2007, BMU.

Federal Ministry for the Environment, Nature Conservation and Nuclear Safety:

Der Indikatorenbericht 2010 zur Nationalen Strategie zur biologischen Vielfalt. 2010, Schottenheim druck\&werbung.

Federal Ministry of Transport, Building and Urban Development: 30-ha-Ziel realisiert. 2011, BMVBS.

Federal Statistical Office: Bodenfläche nach Art der tatsächlichen Nutzung. 2011, Federal Statistical Office.

Fischer, M. and Matthies, D.: RAPD variation in relation to population size and plant fitness in the rare Gentianella germanica (Gentianaceae). 1998, American Journal of Botany 85, p. 811-819.

Forman, R. T. T.: Estimate of the area affected ecologically by the road system in the United States. 2000, Conservation Biology 14, p. 31-35.

Forman, R. T. T. and Deblinger, R. D.: Ecological Road-Effect Zone of a Massachusetts Suburban Highway. 2000, Conservation Biology 14, p. 36-46.

Forman, R. T. T.; Sperling, D.; Bissonette, J. A.; Clevenger, A. P.; Cutshall, C. D.; Dale, V. D.; Fahrig, L.; France, R.; Goldman, C. R.; Heanue, K.; Jones, J. A.; Swanson, F. J.; Turrentine, T. and Winter, T. C.: Road Ecology: Science and Solutions. 2003, Island Press.

Forman, R. T. T. and Sperling, D.: The future of roads: No driving, no emissions, nature reconnected. 2011, Solutions 5, p. 10-23.

Friedrich, J. and Geldermann, J.: Potentielle Auswirkungen von Straßenverkehrstransporten auf die Biodiversität. In: Feit, U. and Korn, H. (eds.): Treffpunkt Biologische Vielfalt 12: Interdisziplinärer Forschungsaustausch im Rahmen des Übereinkommens über die Biologische Vielfalt. 2013, Federal Agency for Nature Conservation, p. 107-113.

German Advisory Council on the Environment: Umwelt und Gesundheit: Risiken richtig einschätzen. 1999, SRU.

Haines-Young, R. and Potschin, M.: Proposal for a Common International Classification of Ecosystem Goods and Services (CICES) for Integrated 
Environmental and Economic Accounting (V1). 2010, Centre for Environmental Management.

Hels, T. and Buchwald, E.: The effects of road kills on amphibian populations. 2001, Biological Conservation 99, p. 331-340.

Henger, R.; Schröter-Schlaack, Ch.; Ulrich, P. and Distelkamp, M.: Flächeninanspruchnahme 2020 und das 30-ha-Ziel: Regionale Verteilungsschlüssel und Anpassungserfordernisse. 2010, Raumforschung und Raumordnung 68, p. 297-309.

Herrmann, M.; Enssle, J.; Süsser, M. and Krüger, J.-A.: Der NABUBundeswildwegeplan. 2007, Wahrlich-Druck.

Hintzsche, M.: Personal communication. 2012, Bonn, December 17, 2012.

Isepy, S.: Personal communication. 2012, Augsburg, December 19, 2012.

Iuell, B.; Bekker, G.J.; Cuperus, R.; Dufek, J.; Fry, G.; Hicks, C.; Hlaváč, V.; Keller, V. B.; Rosell, C.; Sangwine, T.; Tørsløv, N. and Wandall, B. 1. M.: Wildlife and traffic: a European handbook for identifying conflicts and designing solutions. 2003, KNNV Publishers.

Jaarsma, C. F. and Willems, G.: Reducing habitat fragmentation by minor rural roads through traffic calming. 2002, Landscape and Urban Planning 58, p. 125135.

Jaeger, J. A. G.; Bowman, J.; Brennan, J.; Fahrig, L.; Bert, D.; Bouchard, J.; Charbonneau, N.; Frank, K.; Gruber, B. and Tluk von Toschanowitz, K.: Predicting when animal populations are at risk from roads: an interactive model of road avoidance behavior. 2005, Ecological Modeling 185, p. 329-348.

Jaeger, J. A. G.; Fahrig, L. and Ewald, K. C.: Does the configuration of road networks influence the degree to which roads affect wildlife populations? In: Irwin, C. L.; Garrett, P. and McDermott, K. P. (eds.): Proceedings of the international conference on ecology and transportation. 2006, Center for Transportation and the Environment, p. 151-163.

Jordaan, S. M.; Keith, D. W. and Stelfox, B.: Quantifying land use of oil sands production: a life cycle perspective. 2009, Environmental Research Letters 4, p. $1-15$.

Keller, I. and Largiader, C. R.: Recent habitat fragmentation caused by major roads leads to reduction of gene flow and loss of genetic variability in ground beetles. 2003, Proceedings of the Royal Society 270, p. 417-423.

Keller, I.; Nentwig, W. and Largiadèr, C. R.: Recent habitat fragmentation due to roads can lead to significant genetic differentiation in an abundant flightless ground beetle. 2004, Molecular Ecology 13, p. 2983-2994. 
Lassen, D.: Unzerschnittene verkehrsarme Räume in der Bundesrepublik Deutschland. 1979, Natur und Landschaft 10, p. 333-334.

Lassen, D.: Unzerschnittene verkehrsarme Räume über $100 \mathrm{~km}^{2}$ Flächengröße in der Bundesrepublik Deutschland - Fortschreibung 1987. 1987, Natur und Landschaft 12, p. 532-535.

Lindenmayer, D. B.: Foreword. In: Grez, A. A.; Simonetti, J. A. and Bustamante, R. O. (eds.): Biodiversidad en ambientes fragmentados de Chile. 2006, Editorial Universitaria.

Rassmus, J; Herden, C.; Jensen, I.; Reck, H. and Schöps, K.: Methodische Anforderungen an Wirkungsprognosen in der Eingriffsregelung. 2002, Angewandte Landschaftsökologie 51, p. 1-296.

Reck, H.; Thiel-Egenter, C.; Huckauf, A. and Hinsch, H.: Pilotstudie „Wild + Biologische Vielfalt"،. 2009, Stiftung natur+mensch.

Reck, H.; Schultz, B. and Dolnik, C.: Field guide of Holstein Habitat Corridors and the fauna passage Kiebitzholm. 2011, Holsteiner Lebensraum Korridore.

Reijnen, R.; Foppen, R.; Ter Braak, C. and Thissen, J.: The effects of car traf-fic on breeding bird populations in woodland. Reduction of density in relation to the proximity of main roads. 1995, Journal of Applied Ecology 32, p. 187-202.

Roedenbeck, I. A. and Jaeger, J. A. G.: Auf dem Weg zu straßenökologischer Forschung auf Landschaftsebene. 2006, Naturschutz und Landschaftsplanung 38, p. 297-299.

Schupp, D.: Umweltindikator Landschaftszerschneidung: Ein zentrales Element zur Verknüpfung von Wissenschaft und Politik. 2005, Gaia 14, p. 101-106.

Selva, N.; Kreft, S.; Kati, V.; Schluck, M.; Jonsson, B. G.; Mihok, B.; Okarma, H. and Ibisch, P. L.: Roadless and Low-Traffic Areas as Conservation Targets in Europe. 2011, Environmental Management 48, p. 865-877.

Semlitsch, R. D.; Ryan, T. J.; Hamed, K.; Chatfield, M.; Drehman, B.; Pekarek, N.; Spath, M. and Watland, A.: Salamander Abundance along Road Edges and within Abandoned Logging Roads in Appalachian Forests. 2007, Conservation Biology 21, p. 159-167.

Shanley, C. S. and Pyare, S.: Evaluating the road-effect zone on wildlife distribution in a rural landscape. 2011, Ecological Society of America 2, p. 116.

Stoner, D.: The toll of the automobile. 1925, Science 61, p. 56-57.

Van der Grift, E. A.; van der Ree, R.; Fahrig, L.; Findlay, S.; Houlahan, J.; Jaeger, J. A. G.; Klar, N.; Madriñan, L. F. and Olson, L.: Evaluating the effectiveness of road mitigation measures. 2013, Biodiversity and Conservation 22, p. 425-448. 


\section{ARTICLE III}

Friedrich, J., A. Henriquez Brash, M. Aguayo Arias, and J. Geldermann. Traffic Induced Landscape Fragmentation in the Biobío Region of Chile: Analysis and Prospects. Submitted October $21^{\text {st }} 2013$ to: International Journal for Social Ecology and Sustainable Development.

Printed with permission for non-commercial use. 



\title{
Traffic Induced Landscape Fragmentation in the Biobío Region of Chile: Analysis and Prospects
}

\author{
Jan Friedrich $^{1 *}$, Alex Henriquez Brash ${ }^{2}$, Mauricio Aguayo Arias², Jutta Geldermann ${ }^{1}$ \\ ${ }^{1}$ University of Göttingen, Faculty of Economics, Chair of Production and Logistics, Platz der Göttinger Sieben 3, 37073 Göttingen, Germany \\ ${ }^{2}$ Environmental Science Center EULA -Chile, University of Concepcion, casilla 160-C, Concepción, Chile \\ *Corresponding author: jan.friedrich@wiwi.uni-goettingen.de
}

\begin{abstract}
Destruction and fragmentation of habitats are among the greatest threats to biological diversity. In regions with high road densities research is conducted and measures are applied in order to restore a certain degree of landscape permeability. As it is more effective and feasible to avoid fragmentation than to mitigate it, the issue is also relevant for regions with increasing transport intensities. This article presents an analysis of fragmentation of the "Región del Biobío" of Chile. Results reveal that the overall level of fragmentation is moderate. This means that disturbances and threats to individual animals exist, that the up-keeping of minimum viable population sizes is assumed to rarely be impeded, and that genetic variability across the landscape might be negatively affected. There is an opportunity for strategies of avoidance like bundling traffic or supporting sustainable resource use as well as a need for strategies of mitigation to be applied.
\end{abstract}

Keywords: landscape fragmentation, roads, traffic, undissected areas, defragmentation strategies, sustainable development, transportation planning, biodiversity, conservation.

\section{Introduction}

Roads have brought benefits to human societies for decades. However, the image of a road-utility function might look like a bell rather than an incrementally mounting graph. Over the years numerous studies concluded that besides the destruction of habitats, also their fragmentation represented one of the greatest threats to biological diversity worldwide (Forman, 1995, Jaeger, 2000; MA, 2005; Lindenmayer \& Fischer, 2006; Smith \& Smith, 2009). Mostly affected by human induced fragmentation are earth bound species like mammals and amphibians. However, also aquatic and smaller areal species like flying insects or bats, as well as plant species can be severely impacted. Often hydrologic systems like streams and wetlands are also impeded, which means an impact to occur also in downstream ecosystems. The three major impacts of travelled roads on species are road mortality, habitat loss, and fragmentation. Road mortality is the severest problem at the level of the individual animal and it can also become a problem at the population level, if numbers of killed animals are so high as not to allow for the upkeeping of minimum viable population sizes (MVPs) (Oggier, 2007; NABU, 2007). Habitat loss and degradation occur when natural soil is sealed and when supporting construction or disturbing effects like noise, light or emissions extend outwards from a road. Disturbances can repress plant germination and faunal communication, preying or reproduction success and they can reach as far as several kilometers for noise sensitive species (Reck \& Kaule, 1992; Eigenbrod et al., 2009; Reijnen et al., 1997; Barber et al., 
2010; Shanley \& Pyare, 2011). The extension of the so called road-effect zone correlates with traffic volumes and for roads above 10,000 dtv (daily traffic volume) studies suggest for the major share of the impact to occur within the first 200 meters (Reijnen et al., 1997; Biglin \& Dupigny-Giroux, 2006; Forman \& Deblinger, 2000). Road mortality and habitat loss are mentioned here because in addition to their ecological relevancy they can be causes of fragmentation. Fragmentation is both the process and the result of transforming large habitat patches into smaller more isolated fragments of habitat (EEA, 2011). Fragmenting elements of a landscape can be natural features, such as rivers or mountains, and anthropocentric features, such as intensively used agricultural areas, settlements, and linear transport infrastructure (Mader, 1984). A road can represent a barrier due to mortality in the road, deterrence for instance by noise or surface, and due to physical construction. It then impedes animals' daily and yearly movements, including erratic dispersal movements which are important for interchanging genetic traits. Further, it can subdivide a population or a larger meta-population into smaller subpopulations. Populations that are either isolated or smaller than naturally intended face a higher risk of extinction because of possible demographic instability, higher exposure to regional climate events, and reduced genetic variability and the subsequently decreased capability of adaption. Thus, basic connectivity within a landscape or between wildlife corridors is important, because otherwise the stability and long term survival of populations is irreversibly threatened. To summarize, Figure 1 graphically displays the three effects referred to above.

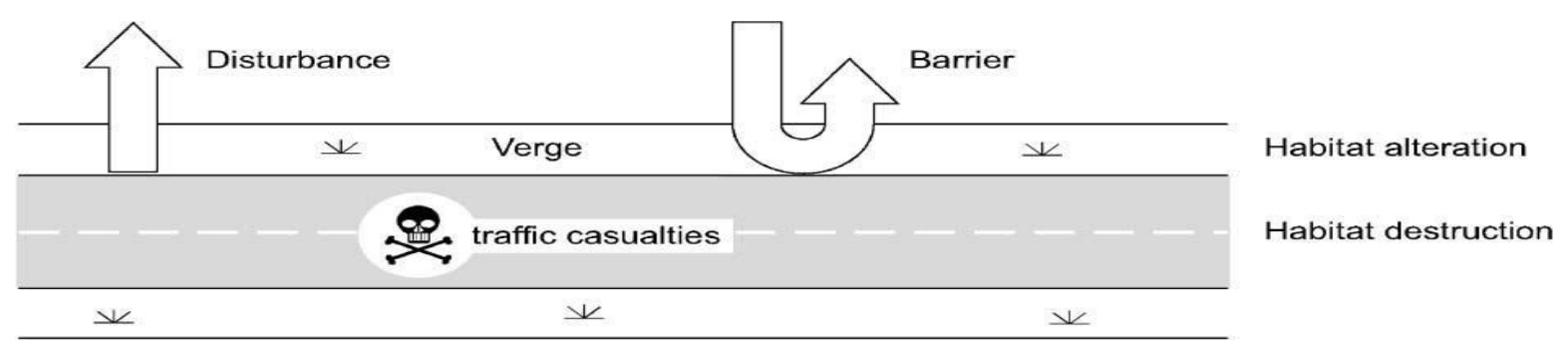

Figure 1. Potential impacts of roads and traffic on flora and fauna. Source: Jaarsma \& Willems, 2002 (C) 2002, Elsevier. Used with permission.).

Consciousness of the issue is particularly high in densely populated and commercially active regions with dense road networks. In Germany, $4.38 \%$ of the terrestrial land mass of $357,127 \mathrm{~km}^{2}$ is sealed by road-related infrastructure and 20 to $50 \%$ of the land is affected for reasons dissimilar to the sealing of soil (Friedrich \& Geldermann, in press). Regarding fragmentation, a national analysis determined over 900 conflicts of priority where ecological connectivity was impeded (NABU, 2007). In Europe, a recent study found Scandinavian and Eastern European countries to be much less fragmented than the countries of central Europe (EEA, 2011). The study's authors advised not to use those countries as benchmarks, because they already faced significant problems of fragmentation. Instead the study recommends not reiterating the mistakes made in central Europe, an estimation that was an incentive to determine the status-quo of fragmentation of the Biobío Region. The authors further argue that avoiding fragmentation would favor not only the conditions of biodiversity in not yet highly fragmented regions but also save respective countries large costs of ex post defragmentation efforts as currently occurring in Germany. Due to the good availability of data and the prospect of economic intensification the results will be carefully compared to the situation in Germany nonetheless. 
Chile as a whole and the Biobío Region itself were assumed to not yet have as dense or as intensively used road grids as central European countries. The two indicators of population density, which is 225 people/ $\mathrm{km}^{2}$ in Germany and 53 people/ $\mathrm{km}^{2}$ in the Biobío Region, and GDP based on purchasing-power-parity per capita, which is 39,028\$US for Germany and 18,419\$US for Chile for 2012, suggest so (INE, 2012; CONAF, 2011; IMF, 2013). However, looking at the development over the last decade and at estimations for the future, both IMF figures of expected economic growth and spatial data identifying urban and industrial area as the land use type of largest relative gain express trends of further urban development and economic intensification and the subsequent extension of the road grid with increasing traffic volumes (CONAF, 2011; IMF, 2013). The Biobío Region and Chile overall are susceptible to human induced fragmentation because despite being large in size $\left(755.696 \mathrm{~km}^{2}\right)$ the territory is very narrow $(4275 \mathrm{~km}$ north to south with an average of $180 \mathrm{~km}$ west to east). It is partitioned horizontally by the Pacific Ocean in the West, the Andes in the East, and the Cordillera de la Costa rising from the sea. Vertically dissecting natural elements are a number of large rivers with diameters of up to 2,000 meters (Rio Biobío). Landscapes with habitat sizes and habitat connectivity that allow for minimum viable population sizes to be kept up and for the possibility for animals to move and disperse would protect wildlife at an individual and populationary level and benefit entire ecosystems. This research analyzes the current degree of fragmentation of the Biobío Region in order to assess whether strategies of mitigation or avoidance can be applied. The analysis considers the entire landscape but is limited to the impacts of larger roads. Other studies exist that deal with the effects of forest roads (Bustamante et al., 2006). For the Biobío Region fragmentation was found to also concern protected areas, which are struggling to conserve wild species due to their sizes and surroundings (Simonetti, 2006). Therefore Simonetti (2006) demanded to increasingly look at landscapes as a whole when analyzing the conditions of biodiversity and its harming triggers. The analysis at hand does just that. Prior to presenting the results the methods and the study area are introduced.

\section{Applied Methods}

\section{Undissected Low Traffic Areas; Effective Mesh Size; Relation Perimeter/ Area}

Over the last twenty-five years, particularly two methods to measure the degree of fragmentation within a landscape became evident. With the measure ULTA100, standing for 'large undissected low-traffic area larger than $100 \mathrm{~km}^{2}$ ', the share of areas fulfilling particular fragmentation geometry is determined (Lassen, 1987). Two strengths of this approach are that firstly the definition of an ULTA $_{100}$ is intuitive, and secondly, that without much further sampling it can be assumed that an undissected area of a certain size is both a prerequisite and a facilitator for the functioning of regional, national, and supranational ecosystems and their species diversity (Turner, 2006; Selva et al., 2011; Baier \& Holz, 2001).

The second internationally well-established fragmentation measure is called effective mesh size $\left(\mathrm{m}_{\mathrm{eff}}\right)$ (Jaeger et al., 2006). It relates to each other the size and the multitude of undissected areas within a total area, so that the result can function as a quantitative expression of landscape connectivity (Reck et al., 2008). As it takes into account the possibility of movement between all points of a given landscape, it expresses the probability of two randomly situated animals to meet each other. In a long term perspective, this is the most basic prerequisite for the persistence of animal populations (EEA, 
2011). Ideally, the $\mathrm{m}_{\mathrm{eff}}$-value calculated for a region would equal the size of that region. This would mean that no fragmenting elements exist and that from a given point all other points in the landscape can be reached. Only in the case of all patches being of identical size does $m_{\text {eff }}$ yield the average size of all patches. One advantage of this measure is that it analyzes a region entirely because it considers patches of all sizes. The $\mathrm{m}_{\text {eff- }}{ }^{-}$ approach is depicted by the following formula published by Jaeger et al., (2006):

$m_{\text {eff }}=\left[\left(\frac{A_{1}}{A_{\text {total }}}\right)^{2}+\left(\frac{A_{2}}{A_{\text {total }}}\right)^{2}+\left(\frac{A_{3}}{A_{\text {total }}}\right)^{2}+\ldots+\left(\frac{A_{n}}{A_{\text {total }}}\right)^{2}\right] \cdot A_{\text {total }}=\frac{1}{A_{\text {total }}} \sum_{i=1}^{n} A_{i}^{2}$

$\mathrm{A}_{\mathrm{i}=1, \ldots, \mathrm{n}}: \quad$ size of the individual areas or patches, 1 through $\mathrm{n}$

$\mathrm{A}_{\text {total }}: \quad$ total size of the investigated region

$\mathrm{n}$ : number of patches of the investigated region

A third measure that is used is the average RPA (Relation Perimeter/ Area) (Pauchard et al., 2006). In relating the perimeter of a patch to its size, the degree of proximity or difficulty to reach all locations within that patch can be determined. Hence, the phenomenon of incisions, which ULTA 100 and $\mathrm{m}_{\mathrm{eff}}$ insufficiently account for, can be assessed. Incisions for instance are roads that end in mountainous areas and which do not fully dissect an area into two parts but which together with changing vegetation can mean a complete barrier for some species. In order to determine their influence, RPA will be calculated for two scenarios, one without, another with incisions.

\section{Fragmentation Geometry}

To calculate the number and the percentage of areas larger than $100 \mathrm{~km}^{2}$, the $\mathrm{m}_{\text {eff }}$-value, and the RPA, the most important definitional aspect is the nature of the fragmentation geometry. Here it is declared which elements are considered to be fragmenting and hence define the limits of an area. The $\mathrm{ULTA}_{100}$ and the $\mathrm{m}_{\mathrm{eff}}$-measures have repeatedly been calculated in Germany and therefore the geometry adopted there was taken as a basis and was then extended to suit the study area (UBA, 2012). In order not to be too ridged with presumed definitions and to detect possible sensitivities, three scenarios with partially differing fragmentation geometries were calculated: I. The baseline scenario, which inter alia assumes 1,000 dtv and only the widest parts of the largest rivers as fragmenting and which corresponds to internationally used geometries (EEA, 2011; LIKI, 2011), II. the precautionary scenario, where traffic volumes being considered as fragmenting are decreased to 500 vehicles per day and rivers of a minimum width of 20 meters are included (Girvetz et al., 2008), and III. the core region scenario, where patches with artificial administrative borders are excluded in order to obtain a result free of the boundary bias (the rest of the geometry is the same as in scenario I). Although natural rivers are not usually considered as being of fragmenting nature, their inclusion is viewed suitable under the precautionary scenario (Jaeger et al., 2006; Moser et al., 2006; Girvetz et al., 2008; EEA, 2011). For scenarios I and II, sub-scenarios labeled Ib and IIb are added where the Andean patches are discounted from the calculation. The reason for examining this situation in both scenarios is due to the area's geography. It is a mountainous region that also in the future will not be intensively used by humans. Due to the size of the area its inclusion is expected to lead to a significant improvement of the results. As an inclusion manipulates the results of the average habitable and arable landscape more than it serves their accuracy the Andean area is omitted under scenarios Ib and IIb. No sub-scenario is needed for the core region scenario as the Andean 
area has administrative borders. The fragmentation geometry exists of the following dissecting elements:

1. Roads with an average dtv of 1,000 or higher (or with a dtv of 500 or higher at some time during the year for scenario II);

2. built up area, usually of urban or industrial use;

3. sections of the major rivers (Río Itata until Nueva Aldea, Rió Nuble until Puerte Ñuble/ Chillán, Río Biobío until Santa Barbara for scenario 1; Río Perquilauquen, Río Nuble until river mouth of Río Los Sauces, Río Itata, Río Diguillin, Río Laja, Río Claro, Río Guaqui, Río Vergara, Río Renaico, Rió Biobío for scenario II; see figure 2 for a graphical display);

4. the borders of the region (not for scenario III);

5. tunnels shorter than 1,000 meters;

6. waterbodies at least as large as Laguna Avendano, which measures $1.08 \mathrm{~km}^{2}$;

7. a first elevation discrepancy of roughly 1,000 meters for the Andean region.

Not considered are railroads, because the very few that exist are not electrified and are not used intensively enough to be of fragmenting nature. The minimum size for patches to be considered is one hectare. In the German fragmentation geometry some rivers are included as barriers mainly due to shipping traffic. Despite none of the rivers of the Biobío Region is navigated, due to their width some rivers were assumed to have a fragmenting effect. Figure 2 shows the three major rivers considered under scenario I as well as all rivers wider than 20 meters assumed as borders of movement under scenario II. Elevation discrepancy is included as a natural border because it applies to species living in lower altitudes and because it allows partially considering the fragmenting character of incisions under the ULTA $_{100^{-}}$and $\mathrm{m}_{\mathrm{eff}}$-metrics (Girvetz et al., 2008). For the calculation of undissected low traffic areas the entire landmass of the region is considered and for the effective mesh size the sum of all habitable patches is used as the total area. The reason for this differentiation is that the ULTA-approach also has a strong habitat-component and hence considers the entire potentially available area while the $m_{\text {eff }}$-approach focuses on the fragmentation of an existing array of habitat patches.

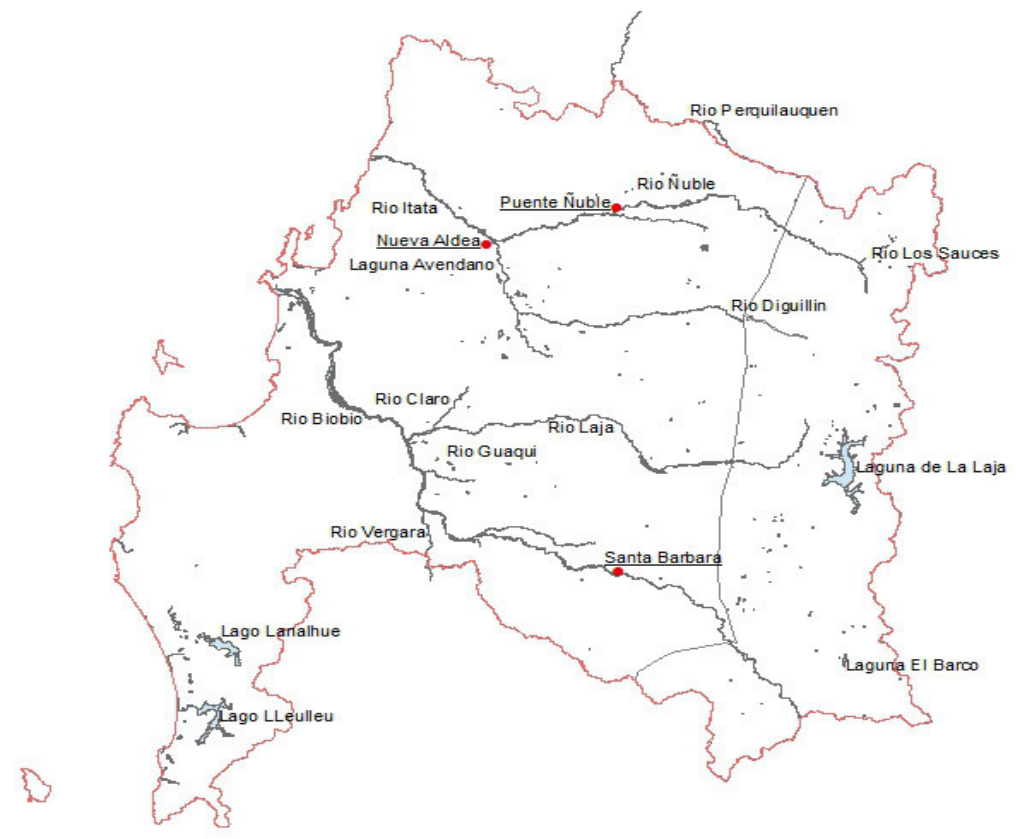

Figure 2. Courses of the major rivers concerned in the baseline and precautionary scenarios I and II 


\section{Sources of Data}

GIS software was used to map the spatial information and to determine and calculate all patches of the Biobío Region. The data needed for the calculation of ULTA $\mathrm{A}_{100}, \mathrm{~m}_{\text {eff }}$, and RPA came mainly from two sources: The spatial and geographical data, which consists of the locations and extensions of cities, roads, and the borders of the region, as well as of the continental and hydrological formation of the region stems from the former Ministerio de Desarrollo y Planificación, which today is the Ministerio de Desarrollo Socíal. The entire register of data is entitled "MIDEPLAN, Año 2002". While the old age of the data is not so much a problem for the geographical features, because they are assumed to have remained largely unchanged, the different land use features like urban, industrial, and infrastructure development are more sensitive and they in turn are expected to have changed to a notable degree since 2002. Therefore a number of different and more recent road maps from the Subdepartamento de SIG y Cartografía de la Dirección Nacional de Vialidad (GIS and Cartography Department of the Ministry of Transport) from 2011 and the Unidad de Sistemas de Información Geográfica - División Regional (Department of Geographical Informationsystems - Regional Division) from 2009 were considered in addition. For urban and industrial area, more recent data came from the Ministerio de Agricultura and its report "Cadaster of Land Use and Vegetation", which was put together by CONAF (National Forestry Authority) and CONAMA (National Environmental Agency). It was published in 2011 and the latest data on the Biobío Region is of the year 2008 (see Table 1). The increase from the GIS formatted MIDEPLAN data of $249 \mathrm{~km}^{2}$ in 2002 to $357 \mathrm{~km}^{2}$ in 2008 was attempted to be accounted for by assuming marginal extensions of build-up area for most locations. Additional topographic information came from an Esri ArcGIS Online Viewer, which was accessed through the Ministerio de Obras Públicas (Ministry of Public Works).

The second main stream of data consists of transit volumes. Data was gathered by the Dirección de Vialidad (Department of Transportation) and was provided by the Departamento Estadísticas y Censos de Tránsito (Department of Statistics and Traffic Census). The data set is of the year 2010 and it is entitled Plan Nacional de Censos - Volumen de Transito Año 2010 (National Traffic Census - Traffic Volumes of the Year 2010).For Germany, data of fragmentation is adjoined in table 2. The value for the country's land mass came from the German Federal Statistical Office (2011). Calculations of ULTA and $m_{\text {eff }}$ were mainly done at the Federal Agency for Nature Conservation (BfN) and in collaboration with the Federal States Initiative (LIKI). The data displayed here describes the state of 2010 (BfN, 2013).

\section{The Study Area}

The Biobío Region has a size of $3,706,003.3$ hectares or $37,060.03 \mathrm{~km}^{2}$. It is located in the central zone of Chile $\left(36^{\circ} 33^{\prime} 06^{\prime \prime} \mathrm{S}-72^{\circ} 45^{\prime} 12^{\prime \prime} \mathrm{O}\right)$. The climate is that of a zone in transition between mediterranean and temperately humid. This favors the development of hard-petalled plant species particularly in northern parts. One major characteristic of the region are the coastal and the Andean mountain ranges, which together with the climate and proximity of the sea produce a dense hydrologic system consisting of many smaller and fewer very large rivers like the Río Nuble, Río Diguillin, Río Itata, Río Biobío and Río Laja. Also, two ecological regions of globally prioritized protection status, the mediterranean coppice and the template rainforest, are part of the natural vegetal cover (Aguayo et al., 2009). Due to their large spatial extension, forest plantations are a dominant characteristic of the Biobío Region. About $60 \%$ of the over 2 million hectares 
of wood land are plantations of exotic species of fast growth, mainly eucalyptus and pine. They hence cover $33.1 \%$ of the region's total surface. This large scale change of natural surface means a significant decrease of the abundance of native flora and fauna as such and it further is an example how drastic agricultural or forestal land use change is of fragmenting impact as well because the majority of native species neither lives nor moves and disperses through this type of vegetation. Despite the intensive use of areas of forest plantations, some species can be expected to adapt and to live in and roam through them. However, species richness will be nowhere near the levels of that of native forests. For the study at hand, this has two significant impacts: Firstly, the fragmenting effect of a road is not as high in areas where the road is surrounded by plantations because there is less life to be bothered. Secondly, the road is not the dominant element of fragmentation because the barrier effect already is established by the alien vegetation. Despite the relevancy of the former, the analysis at hand is limited to roads. A continuative study that dealt with the habitat quality and fragmenting effect of plantations in more detail, and that incorporated the findings presented here could help identifying and prioritizing the most important conflicts of fragmentation.

Other major surface covers are agriculture and areas without vegetation with 8.3 and 1.3 million hectares respectively. Urban and industrial areas account for $357 \mathrm{~km}^{2}$, which is just below 1\%. In Germany, this category takes up 13.4\% of the land (Federal Statistical Office, 2011). Increases in land use from the year 1998 to 2008 occurred in urban and industrial area and in woodland. These are the categories with the most intensive human impact, because for woodland plantations increased by $2,691 \mathrm{~km}^{2}(14.8 \%)$, while the amount of native forest area remained fairly constant as native forests were cut back by $78 \mathrm{~km}^{2}(1 \%)$. Urban and industrial area recorded the largest relative gain as they augmented by $38.3 \%\left(99 \mathrm{~km}^{2}\right)$. Gains came at losses mainly of grassland and coppice as well as agricultural area, with $1,387 \mathrm{~km}^{2}$ and $1,356 \mathrm{~km}^{2}$ respectively (CONAF, 2011). Table 1 displays the values of all land use types for the years 1998 and 2008. Overall, the numbers speak of ongoing trends of urban development and economic intensification.

Table 1: Land Use in the Biobío Region, Years 1998 and 2008

\begin{tabular}{|l|c|c|c|c|c|r|r|r|c|c|}
\hline $\begin{array}{l}\text { Type of } \\
\text { surface }\end{array}$ & $\begin{array}{c}\text { Urban } \\
\text { area }\end{array}$ & $\begin{array}{c}\text { Agricul- } \\
\text { ture }\end{array}$ & $\begin{array}{c}\text { Grassland } \\
\text { and coppice }\end{array}$ & Woodland & $\begin{array}{c}\text { Wet- } \\
\text { lands }\end{array}$ & $\begin{array}{c}\text { Areas without } \\
\text { vegetation }\end{array}$ & $\begin{array}{c}\text { Snow- and } \\
\text { icefields }\end{array}$ & $\begin{array}{c}\text { Water } \\
\text { Bodies }\end{array}$ & Other & Total \\
\hline $\begin{array}{l}\text { Size in } \\
\mathrm{km}^{2} 1998\end{array}$ & 258.03 & $9,651.45$ & $6,371.63$ & $17,884.27$ & 120.28 & $1,339.76$ & 906.92 & 525.58 & 2.11 & $37,060.03$ \\
\hline $\begin{array}{l}\text { Size in } \\
\mathrm{km}^{2} 2008\end{array}$ & 356.86 & $8,295.08$ & $4,984.96$ & $20,529.82$ & 115.95 & $1,325.18$ & 904.68 & 547.42 & 8.10 & $37,060.03$ \\
\hline $\begin{array}{l}\text { Size in \% } \\
\text { in 2008 }\end{array}$ & 1.0 & 22.1 & 13.2 & 55.8 & 0.3 & 3.6 & 2.5 & 1.5 & 0 & 100 \\
\hline
\end{tabular}

Source: Adapted from Catastro de Uso del Suelo y Vegetación, CONAF, 2011.

With regard to the faunal species that are potentially affected by overland roads various mammalian species like coipo (Myocastor coypus), viscacha (Lagidium viscacia), guiña (Leopardus guigna), or zorro de Darwin (Lycalopex fulvipes) are identified. Furthermore, many amphibian species are concerned.

\section{Results and Discussion}

As indicated in the fragmentation geometry, three main scenarios are assumed in the analysis. Compared to the baseline scenario, under the precautionary scenario stricter assumptions on the dissecting character of an element are made with regard to traffic 
volumes and the width of rivers. As it is disadvantageous that two parameters change between the scenarios, it is constituted that the subsequent moderate change in the results of the two scenarios is provoked more so by added rivers than it is by added roads. Sub-scenarios Ib and IIb are added because discounting the large sized and very extensively used Andean patches, to date and for the medium-term future, yields more representative results for the area under human interference and stewardship. Due to the omittance of the inappropriately designated boundary areas and due to the probably sufficiently strict fragmentation geometry of scenario I, results of the core region scenario are viewed to be the most resilient ones. This is in spite of the fact that the area considered by this scenario covers only half of the Biobío Regions' entire landmass. The following table gives a concise overview of the numeric results. The results are described and interpreted in the subsequent paragraphs.

Table 2: Results of the Analysis of Fragmentation of the Biobío Region of Chile

\begin{tabular}{|c|c|c|c|c|c|c|c|}
\hline \multirow[b]{2}{*}{ Scenarios } & \multicolumn{4}{|c|}{ ULTA $_{100}$} & \multicolumn{3}{|c|}{$\mathbf{m}_{\text {eff }}$} \\
\hline & total area & $\begin{array}{l}\text { ULTA cov- } \\
\text { er }\end{array}$ & in $\%$ & $\begin{array}{c}\text { ULTA }_{500} \\
\text { in } \%\end{array}$ & total area & $\mathrm{km}^{2}$ & $S_{\text {eff }}$ \\
\hline $\begin{array}{l}\text { Ia: } \geq 1000 \mathrm{dtv} ; \\
\text { large rivers }\end{array}$ & $37,060.03$ & $35,259.32$ & $95.14 \%$ & $85.72 \%$ & $35,919.99$ & $3,387.19$ & 3 \\
\hline $\begin{array}{l}\text { Ib: Ia without } \\
\text { Andean patch }\end{array}$ & $27,806.70$ & $26,090.26$ & $93.54 \%$ & $81.03 \%$ & $26,750.93$ & 779.49 & 13 \\
\hline $\begin{array}{l}\text { IIa: } \geq 500 \mathrm{dtv} \\
\text { rivers }>20 \mathrm{~m}\end{array}$ & $37,060.03$ & $34,448.77$ & $92.95 \%$ & $76.76 \%$ & $35,883.53$ & $2,732.51$ & 3.7 \\
\hline $\begin{array}{l}\text { IIb: IIa without } \\
\text { Andean patches }\end{array}$ & $27,806.70$ & $25,289.28$ & $90.95 \%$ & $73.20 \%$ & $26,724.04$ & 675.97 & 15 \\
\hline $\begin{array}{l}\text { III: Ia without } \\
\text { boundary areas }\end{array}$ & $18,253.38$ & $16,878.52$ & $92.47 \%$ & $75.95 \%$ & $17,539.19$ & $1,056.94$ & 9.5 \\
\hline Germany 2010 & $357,127.00$ & $82,702.00$ & $23.16 \%$ & $1.00 \%$ & $357,127.0$ & 82.02 & 122 \\
\hline
\end{tabular}

Regarding the analysis of undissected low traffic areas larger than $100 \mathrm{~km}^{2}$, results for all five scenarios (Ia;Ib;IIa;IIb;III) yielded values above $90 \%$ of the region's total area to consist of areas of at least $100 \mathrm{~km}^{2}$. This means that areas sized $100 \mathrm{~km}^{2}$ or larger are no scarcity but make for the majority of the mostly semi-natural landmass of the Biobío Region. Enlarging the size of undissected low traffic areas to $500 \mathrm{~km}^{2}$, even for the scenario with the strictest rules of fragmentation those areas still make for $73 \%$ of the landscape. Differences between the scenarios are moderate. When omitting the Andean patch(es) values fall about $2 \%$ (95.14 to $93.54 \%$ and 92.95 to $90.95 \%$ ). Not accounting for patches that are delimited only by administrative boundaries yields a value of $92.47 \%$. This shows that the high values obtained here are not dictated by large boundary patches, but that also in the center of the region the majority of natural patches exceeds $100 \mathrm{~km}^{2}$. ULTA shares of Germany are at the other end of the spectrum. Particularly scarce are very large areas as they make for only $1 \%$ of the landmass. These numbers tell of the worthiness that such areas have in a heavily fragmented landscape.

With regard to the effective mesh size of a landscape, the best $m_{\text {eff-value from a }}$ defragmentation perspective is $\mathrm{m}_{\mathrm{eff}}$-max, equaling $\mathrm{A}_{\text {total }}$. $\mathrm{A}$ larger $\mathrm{m}_{\mathrm{eff}}$-value means less fragmentation. Values of $m_{\text {eff }}$ can better be comprehended when applying one further step of calculation, which converts values of $m_{\text {eff }}$ into values of the effective mesh density $\left(\mathrm{s}_{\mathrm{eff}}\right)$. $\mathrm{S}_{\mathrm{eff}}$ is the effective number of meshes per given square unit. For the calcula- 
tion of $s_{\text {eff }}$ the assumed size of a unit has to be divided by the $m_{\text {eff }}$-value. The size of the unit in this case is assumed to be $10,000 \mathrm{~km}^{2}$. Thus, $\mathrm{s}_{\text {eff }}$ of scenario Ia is 2.95 $(10,000 / 3,387=2.95)$. This means that an area of $10,000 \mathrm{~km}^{2}$ contains nearly three meshes. The result of the most resilient scenario III yields 9.5 meshes per $10,000 \mathrm{~km}^{2}$. Compared to the situation in Germany, where a unit of $10,000 \mathrm{~km}^{2}$ contains 122 patches, the obtained value appears to be rather low (and the German $s_{\text {eff-value in fact is even }}$ greater because in the calculations Germany's entire land mass was considered as $A_{\text {total }}$ and not the sum of patches). As expected, higher numbers appear for the scenarios without the Andean patch, Ib and IIb, with 13 and 15 patches respectively per 10,000 $\mathrm{km}^{2}$. This is a larger difference compared to the only moderate change when deducing the Andean patch under the ULTA 100 -metric. It shows that large areas have a dispropor-

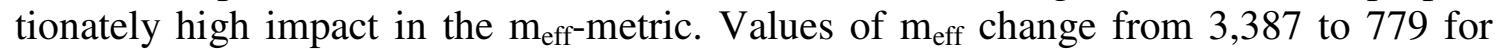
scenario I and from 2,733 to 676 for scenario II, while under ULTA 100 they drop from 95 to 93 and 93 to $91 \%$ respectively. Similarly, regarding the comparison of the core region scenario III to scenario Ia, the seemingly small difference in results in the case of ULTA $_{100}(92$ from $95 \%$ ) in the case of the effective mesh size turns into a more significant difference $\left(1,057\right.$ to $\left.3,387 \mathrm{~km}^{2}\right)$. The $\mathrm{m}_{\mathrm{eff}}$-results hence indicate a substantial increase in fragmentation for the scenario without boundary areas. This means that the landscape is more fragmented in the center of the region and that ULTA 100 was a measure not ambitious enough to detect so. This is supported by the results of the ULTA $500^{-}$ case, which begin to indicate a larger difference of fragmentation levels between the two scenarios (baseline scenario 86 vs. core region scenario $76 \%$ ). Overall, the $\mathrm{m}_{\text {eff- }}{ }^{-}$ results are more precise and they reflect the location of the region's three major cities (Chillán, Concepción, Los Àngeles) and the related infrastructure density around and between them, as none of them is situated in a boundary area (see Figures 3 and 4d). Various studies discovered this tendency, that land-cover change and landscape fragmentation are often inversely related to the distance to urban centers (EEA, 2011). Not having a socioeconomic cause but being a partial reason for socioeconomic development most of the larger rivers also have their fragmenting effect in the center of the region (cp. Figure 2).

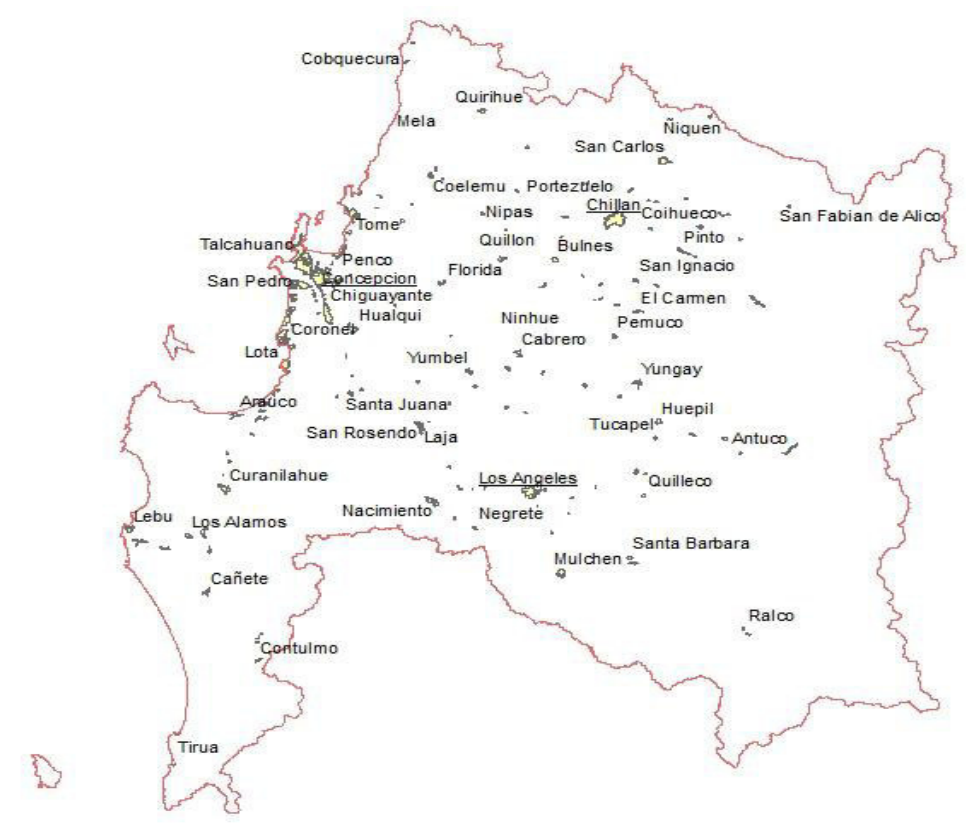

Figure 3. Location of larger cities in the Biobío Region 
With regard to the suitability of the methods of $\mathrm{m}_{\mathrm{eff}}$ and $\mathrm{ULTA}_{100}$, the results show that for a more detailed analysis of fragmentation at a landscape level, the $\mathrm{m}_{\text {eff-methodology }}$ is superior to the ULTA-metric. As this is the first calculation of $\mathrm{m}_{\mathrm{eff}}$, no trends could be investigated but the results can form a basis to do so in future analyses. To conclude, figures $4 \mathrm{a}$ to $4 \mathrm{~d}$ give a graphical representation of the previously discussed results. The chief results of an intensity of use of the road grid that does considerably dissect the landscape, but so far partitions it into rather few patches of relatively large sizes, as well as the tendency of higher degrees of fragmentation towards the center of the region, are mirrored in the maps.

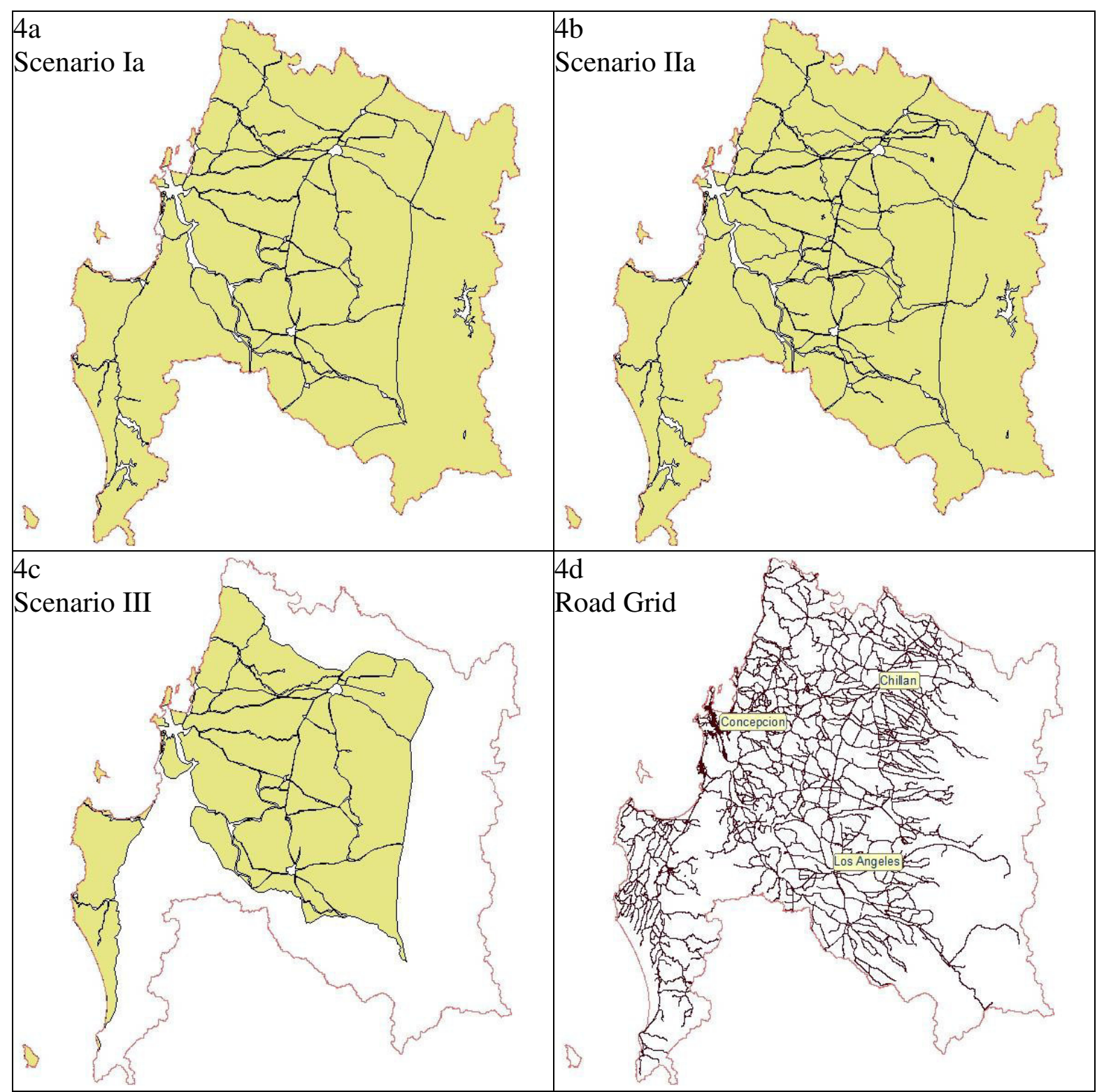

Figures 4a. Patches of 1,000 dtv and large rivers (Sc. Ia); 4b. Patches of $500 \mathrm{dtv}$ and rivers wider than 20 meters (Sc. IIa); 4c. Area considered when leaving out boundary patches (Sc. III); 4d. Grid of larger roads.

In order to analyze the influence of incisions in addition to abandoning the incision-rich Andean patch(es), the RPA-metric was applied. As the RPA-values when excluding and next including incisions were not expected to differentiate notably between the various scenarios, the calculation of RPA was carried out only for the most resilient core region scenario. The results in table 3 show that including incisions into the perimeter does augment the RPA-value. However, the increase from 1,438 to 1,442 meters of average 
perimeter per one square kilometer of area is very low. This means that while individual incisions, as for instance the one near Los Alamos (see figures 2 and 4), do impact animal movement and other natural flows in specific parts of the landscape, the overall impact at the level of the region is not large enough to constitute a major spatial issue to a rather permeable landscape. Again, this interpretation is valid only for ecosystem components impacted by larger roads and thus the effects of dissimilar types of roads are expected to differ (Liu et al., 2008).

Table 3: Average Relation Perimeter/Area for the Biobío Region

\begin{tabular}{|l|l|l|}
\hline & \multicolumn{2}{|c|}{ Relation Perimeter/Area (RPA) } \\
& without incisions & with incisions \\
\hline Scenario III: no boundary areas & $1,438.57$ meters per $\mathrm{km}^{2}$ & $1,442.44$ meters per km² \\
\hline
\end{tabular}

\section{Limitations and Recommendation}

One definitional limitation in the way that undissected low traffic areas and effective mesh size and density were calculated is the assumption of the region's limits as boundaries. It is inaccurate because an administrative border does not represent a factual barrier for flora and fauna. In literature, calculating degrees of fragmentation under the supposition of administrative borders to represent barriers is called cutting-out procedure (Jaeger et al., 2006). In an alternative approach, which is called cross-boundary connections procedure, borders are not considered to fragment patches, only physical barriers are (Moser et al., 2007). The difficulty here is that in order to determine patch sizes, data of the neighboring regions or national entities need to be known. The scope of the analysis at hand did not permit to gather that data. To address the problem of borders, in scenario III all boundary patches were discarded from the calculation.

Another limitation is the definition of 1,000 and 500 vehicles per day to be of fragmenting impact. However, it is preferred to assume a barrier that factually is no barrier for particular larger mammalian species than not to assume a barrier when a barrier is present to a significant number of species. With regard to roads representing complete barriers to all species, very few roads in the region exist that carry more than 10,000 vehicles per day. Finally, the analysis draws no complete picture of the problem of fragmentation, because smaller roads and areas under intensive use were not investigated.

The calculations of $m_{\text {eff }}$ and $s_{\text {eff }}$ are particularly sensitive to the number of patches that has been determined in the study. Despite the facilitating assumption to consider only patches larger than one hectare, future calculations that might use slightly different geometries at this point must pay attention to the question whether comparability with the present results is given. The numbers of patches under the different scenarios are listed in Table 4.

Table 4: Numbers of Patches Going into Effective Mesh Size Calculations

\begin{tabular}{|l|l|l|l|l|l|}
\hline & Scenario Ia & Scenario Ib & Scenario IIa & Scenario IIb & Scenario III \\
\hline Number of patches & 63 & 62 & 107 & 109 & 55 \\
\hline
\end{tabular}

When attempting to avoid or to mitigate the two major spatial ecological impacts of road traffic, land use and fragmentation, a number of options exist. Land use can be brought down by choosing more area efficient modes of transportation, such as trains or 
shared road-bound vehicles. While shared vehicles usually perform better with regard to all environmental impacts, their impact is not zero. Hence, their efficiency becomes of limited use when transport volumes steadily grow and pass sustainably transportable amounts. Transport volumes in Germany are approximating if not surpassing this threshold. In Germany, using more efficient modes of transport is becoming an obligation whereas it seems that in Chile it still is an open choice. Barring the lever of transport volumes, the ecological stresses from fragmentation can most effectively be reduced by bundling traffic on fewer roads. This leads to lower road densities as some roads can be upgraded and others be degraded, decommissioned, or even be deconstructed. With regard to transport volumes and their conditioning societal pressures, both impacts of land use and fragmentation are diminished by lowering the transport intensity in production, distribution, and consumption, and also in individual recreational activities. This can be attained by fostering regional economic structures that imply lower haulage distances. Also, lowered transport intensities could be a consequence of new and more total economic value-orientated policy making that passes on and internalizes to date external costs of transport to a higher degree. Or, it could derive from decreased demands, e.g. from citizens desiring to live more self-sufficient and environmentally sustainable, and to be rooted and interacting more in their home communities. This means that at the landscape level central roads to be used have to be identified and their usage to be encouraged. Collecting toll on larger roads and using real time navigation systems are examples of opposing incentives. Along the selected major roads, priority locations for defragmentation have to be identified. In addition to small crossing structures the construction of larger bridges of at least 50 meters in width is believed to be an effort of mitigation that can establish a significant degree of connectivity across essential and busy roads (figure 5).

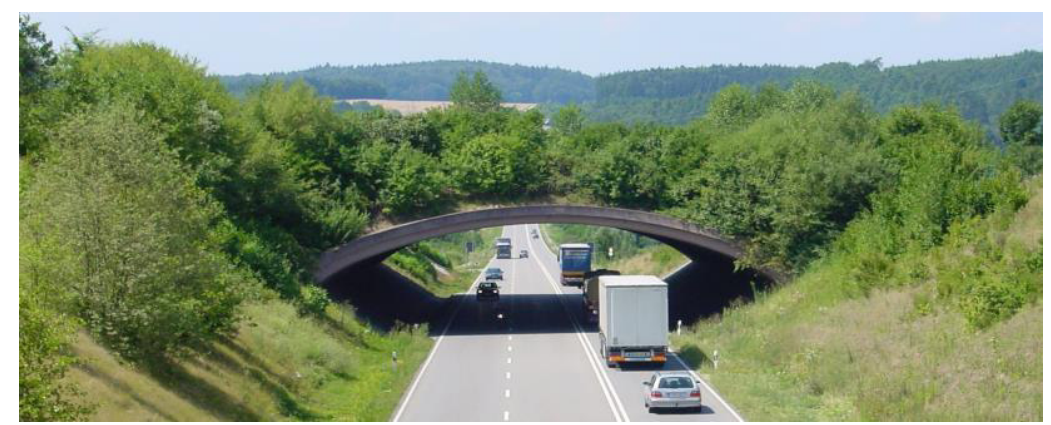

Figure 5. Image of a wildlife overpass; picture by Martin Strein, FVA Freiburg

While the use of wildlife overpasses has been well-recorded, few studies confirm their effectiveness to mitigate population level effects, such as genetic depletion (van der Ree et al., 2009; Corlatti et al., 2009). Reck et al. (2011) elucidate details on the constitution of the surroundings and van der Grift et al. (2013) propose how to best evaluate functionality. Additional artificial structures, which support the movement of wildlife and the flow of water patterns, such as small amphibian tunnels or culverts, can also be implemented. Roads and roadsides can further be improved by drawing from an array of further options of mitigation. Soil berms can be built, quieter surfaces be employed, or driving speeds be reduced in order to diminish the emergence and impact of noise. Also, creating woodier roadsides with complementary fences and traffic signs can increase habitat and decrease the barrier effects for some species (Forman \& Sperling, 2011). For any scenario of future landscape and road use, where it is still uncertain how to establish sufficient permeability, the target to maintain large unfragmented areas seems paramount (Selva et al., 2013; Friedrich \& Geldermann, in press). Shares of ULTA 100 s of 
over $90 \%$ in the Biobío Region tell of a limited level of fragmentation and imply significant opportunity. It is in large areas where ecological processes and species compositions can best follow natural flows. They can be expected to keep the surroundings and enablers of human life as rich and resilient as mankind has experienced them in the past.

\section{Conclusion}

Since the ability of animals to move through the landscape has profound impacts on ecological phenomena and processes, and since dense road networks are a significant anthropogenic cause impeding necessary movements, the goal of this analysis was to determine the current degree of fragmentation of the "Región del Biobío" in order to assess the severity of the impact, to introduce a metric to be able to compare against future developments, and to suggest possible approaches of improvement. Results seem particularly relevant to spatial planners and political leaders. The sealing of natural soil and the loss and degradation of adjunct habitat, as well as physical obstacles and mortality in the road can deter animals from otherwise desired movements. Also, plant distribution and hydrologic systems can be impacted and the overall compromise of natural flows can have a severe influence on individual lives, on the up-keeping of minimal viable population sizes and their genetic variability, and also on local ecosystems. As metrics to analyze the fragmentation of the Biobío Region, the three approaches of undissected low traffic areas larger than $100 \mathrm{~km}^{2}\left(\mathrm{ULTA}_{100}\right)$, the effective mesh size $\left(\mathrm{m}_{\mathrm{eff}}\right)$, and the relation perimeter/area (RPA) were chosen.

Results show that the existing road infrastructure and its current intensity of use in the Biobío Region can be expected to have a significant impact only on individual animals and individual species. The landscape at the center of the region is more densely dissected. Fragmenting elements do not critically diminish the availability of habitat for many species nor do they dissect the landscape into patches of sizes where many animal species cannot live in or maintain MVPs. Yet, degrees of dispersion, density, and intensity of road use are reached that at the level of individual animals or populations render certain areas inhabitable for some species, cause their death in collisions, and might limit gene flows. To alleviate these impacts, apart from identifying and tackling existing priority locations of defragmentation, for the region as a whole ecologically superior strategies of avoidance like bundling traffic and fostering less mobility demanding economic and social organization rather than strategies of costly and disputably effective strategies of mitigation could be pursued. The above assessment is only valid for species that are affected by large road infrastructure and its use. The impacts of minor roads, forest roads, and intensively used semi-natural areas like plantations are not examined. They are addressed in part in studies by Brehme et al. (2013), Semlitsch et al. (2007), and Grez et al. (2006). Comprehensive landscape analyses are rare but could deliver more accurate results on the reasons and level of fragmentation.

It seems helpful to point out that grading impacts and levels of fragmentation, for instance as high or low, is largely subjective because apart from the consensus of undesired species extinction broad societal discussion and consciousness of values towards nature and its diversity have not been important themes in anthropocentrically natured societies. However, rising imminence and extinction of life forms and ecosystem services begin to decrease welfare and happiness and might urge mankind to again think of its relation to non-human nature. Concluding, results of the analysis lead to a proposal that is far from being a claim not to build further roads in the Biobío Region, particularly at a time when the understanding of economic and social development depends on 
inexpensive and fast traffic infrastructure. Identifying the current condition of landscape fragmentation in the region and briefly describing respective ecological impacts may allow for better informed and more conscious choices when enhancing or alleviating the fragmentation of habitats in the future.

ACKNOWLEDGEMENTS: We thank our colleagues from the interdisciplinary PhD program "Biodiversity and Society" at Göttingen University for their input, we thank the management team and employees of the Environmental Science Center EULA Chile at Concepcion University for their support, and we last but not least thank the German Federal Ministry of Education and Research for the financial aid.

\section{Bibliography}

Aguayo, M., Pauchard, A., Azócar, G., \& Parra, O. (2009). Land use change in the south central Chile at the end of the 20th century. Understanding the spatio-temporal dynamics of the landscape. Revista Chilena de Historia Natural, 82, 361-374.

Baier, H., \& Holz, R. (2001). Landschaftszerschneidung als Naturschutzproblem: Die Wirkungen und ihre Vermeidungsstrategien. Naturschutzarbeit in Mecklenburg-Vorpommern, 44, 11-27.

Barber, J.R., Crooks, K.R. \& Fristrup, K.M. (2010). The costs of chronic noise exposure for terrestrial organisms. Trends in Ecology and Evolution, 25, 180-189.

Biglin, K., \& Dupigny-Giroux, L.A. (2006). Mapping the road-effect zone to assess impacts of proposed road segments. Journal of Conservation Planning, 2, 1-16.

BFN (Federal Agency for Nature Conservation). (2013). Ermittlung der Unzerschnittenen Verkehrsarmen Räume > 100 km² für Deutschland 2010. Leipzig: BfN.

BMU (Federal Ministry for the Environment, Nature Conservation and Nuclear Safety). 2010. Der Indikatorenbericht 2010 zur Nationalen Strategie zur biologischen Vielfalt.

Eichenau: Schottenheim druck\&werbung.

BMU (Federal Ministry for the Environment, Nature Conservation and Nuclear Safety). 2011. National strategy on biological diversity, 3rd Edition. Berlin: BMU.

Brehme, C.S., Tracey, J.A., Mcclenaghan, L.R., \& Fisher, R.N. (2013). Permeability of roads to movement of scrubland lizards and small mammals. Conservation Biology, 27, 710-20.

Bustamante, R.O., Grez, A.A., \& Simonetti, J.A. (2006). Efectos de la fragmentación del bosque maulino sobre la abundancia y la diversidad de especies. In A.A. Grez, J.A. Simonetti, \& R.O. Bustamante (Eds.), Biodiversidad en Ambientes Fragmentados de Chile: Patrones y Procesos a Diferentes Escalas (pp. 83-98). Santiago: Editorial Universitaria.

CONAF (Corporación Nacional Forestal) and CONAMA (Comisión Nacional de Medio Ambiente). (2001). Catastro del uso del suelo y vegetación. Santiago: Departamento de Fiscalización y Monitoreo de Ecosistemas Forestales.

Corlatti, L., Hackländer, K., \& Frey-Roos, F. (2009). Ability of wildlife overpasses to provide connectivity and prevent genetic isolation. Conservation Biology, 23, 548-556. 
DEPARTAMENTO ESTADÍSTICAS Y CENSOS DE TRÁNSITO (Department of Statistics and Traffic Census). 2013. Plan Nacional de Censos - Volumen de Transito Año 2010. Retrieved June 25, 2013, from http://servicios.vialidad.cl/censo/index.htm.

EEA (European Environmental Agency). (2011). Landscape fragmentation in Europe Joint EEA-FOEN report. Copenhagen: EEA.

Eigenbrod, F., Hecnar, St. J., \& Fahrig, L. (2009). Quantifying the road-effect zone: threshold effects of a motorway on anuran populations in Ontario, Canada. Ecology and Society, 14, 1-18.

ESRI. (2013). ArcGIS Online Viewer. Retrieved June 25, 2013, from http://www.arcgis.com/home/webmap/viewer.html?url=http\%3a\%2f\%2fwservicesit.mop.gov.cl\%2fArcGIS\%2frest\%2fservices\%2fDV\%2fPNC\%2fMapServer\&source= sd

Federal Statistical Office. (2011). Bodenfläche nach Art der tatsächlichen Nutzung. Wiesbaden: Federal Statistical Office.

Forman, R.T.T. (1995). Land Mosaics: The Ecology of Landscapes and Regions. Cambridge: Cambridge University Press.

Forman, R.T.T., \& Deblinger, R.D. (2000). Ecological Road-Effect Zone of a Massachusetts Suburban Highway. Conservation Biology, 14, 36-46.

Forman, R.T.T., Sperling, D. (2011). The future of roads: No driving, no emissions, nature reconnected. Solutions, 5, 10-23.

Friedrich, J., \& Geldermann, J. (in press). Current practices of mitigating the ecologically harmful effects of roads - An assessment. In J. Friedrich, A. Halsband, \& L. Minkmar (Eds.), Conference Proceedings of Biodiversity and Society. Societal dimensions of the conservation and utilization of biological diversity. Goettingen: Goettingen University Press.

Girvetz, E.H., Thorne, J.H., Berry, A.M., \& Jaeger, J.A.G. (2008). Integration of landscape fragmentation analysis into regional planning: A statewide multi-scale case study from California, USA. Landscape and Urban Planning, 86, 205-218.

Grez, A.A., Simonetti, J.A., \& Bustamante, R.O. (2006). Biodiversidad en Ambientes Fragmentados de Chile: Patrones y Procesos a Diferentes Escalas. Santiago: Editorial Universitaria.

IMF (International Monetary Fund). (2013). World economic outlook database, April 2013. Washington, D.C: International Monetary Fund.

INE (Instituto Nacional de Estadísticas). (2012). Censo 2012. Síntesis de resultados. Santiago: Instituto Nacional de Estadísticas.

Jaarsma, C.F., \& Willems, G. (2002). Reducing habitat fragmentation by minor rural roads through traffic calming. Landscape and Urban Planning, 58, 125-135.

Jaeger, J.A.G. (2000). Landscape division, splitting index, and effective mesh size: new measures of landscape fragmentation. Landscape Ecology, 15, 115-130. 
Jaeger, J.A.G., Esswein, H., \& Schwarz von Raumer, H.G. (2006). Landschaftszerschneidung in Baden-Württemberg: Fortschreibung zeigt weitere starke $\mathrm{Zu}$ nahme der Zerschneidung an. Bad Boller Skripte, 3, 17-48.

Lassen, D. (1987). Unzerschnittene verkehrsarme Räume über $100 \mathrm{~km}^{2}$ Flächengröße in der Bundesrepublik Deutschland - Fortschreibung 1987. Natur und Landschaft, 12, 532 535 .

LIKI. (2011). Landscape fragmentation. Retrieved June 25, 2013, from http://www.lanuv.nrw.de/liki-newsletter/index.php?indikator=13\&aufzu=0\&mode=indi

Lindenmayer, D., \& Fischer, J. (2006). Habitat fragmentation and landscape change: an ecological and conservation synthesis. Washington, D.C.: Island Press.

Liu, S.L., Cuia, B.S., Donga, S.K., Yanga, Z.F., Yanga, M., \& Holt, K. (2008). Evaluating the influence of road networks on landscape and regional ecological risk - A case study in Lancang River Valley of Southwest China. Ecological Engineering, 34, 91-99.

MA (Millennium Ecosystem Assessment). (2005). Ecosystems and Human Well-being: Synthesis. Washington, D.C.: Island Press.

Mader, H.J. (1984). Animal habitat isolation by roads and agricultural fields. Biological Conservation, 29, 81-96.

Ministerio de Desarrollo y Planificación. (2002). MIDEPLAN. Santiago. Data can be made available upon request.

Moser, B., Jaeger, J.A.G., Tappeiner, U., Tasser, E., \& Eiselt, B. (2007). Modification of the effective mesh size for measuring landscape fragmentation to solve the boundary problem. Landscape Ecology, 22, 447-459.

NABU (Nature and Biodiversity Conservation Union). (2007). Der NABUBundeswildwegeplan. Meckenheim: Warlich-Druck.

Oggier, P., Righetti, A., \& Bonnard, L. (2007). Zerschneidung von Lebensräumen durch Verkehrsinfrastrukturen COST 341, 2nd Edition. Bern: Bundesamt für Umwelt; Bundesamt für Raumentwicklung; Bundesamt für Verkehr \& Bundesamt für Straßen.

Pauchard, A., Aguayo, M., \& Alaback, P. (2006). Cuantificando la fragmentación del paisaje: las métricas y sus significados ecológicos. In A.A. Grez, J.A. Simonetti, \& R.O. Bustamante (Eds.), Biodiversidad en Ambientes Fragmentados de Chile: Patrones y Procesos a Diferentes Escalas (pp. 41-68). Santiago: Editorial Universitaria.

Reck, H., \& Kaule, G. (1992). Straßen und Lebensräume - Ermittlung und Beurteilung straßenbedingter Auswirkungen auf Pflanzen, Tiere und ihre Lebensräume. Bonn: Typo-Druck.

Reck, H., Hänel, K., Jessberger, J. \& Lorenzen, D. (2008). ULTA, UFR + Biologische Vielfalt. Bonn-Bad Godesberg: Federal Agency for Nature Conservation.

Reck, H., Schultz, B., \& Dolnik, C. (2011). Field guide of Holstein Habitat Corridors and the fauna passage Kiebitzholm. Molfsee: Holsteiner Lebensraum Korridore. 
Reijnen, R., Foppen, R., \& Veenbaas, G. (1997). Disturbance by traffic of breeding birds: evaluation of the effect and considerations in planning and managing road corridors. Biodiversity and Conservation, 6, 567-581.

Selva, N., Kreft, S., Kati, V., Schluck, M., Jonsson, B.G., Mihok, B., Okarma, H., \& Ibisch, P.L. (2011). Roadless and Low-Traffic Areas as Conservation Targets in Europe. Environmental Management, 48, 865-877.

Semlitsch, R.D., Ryan, T.J., Hamed, K., Chatfield, M., Drehman, B., Pekarek, N., Spath, M., \& Watland, A. (2007). Salamander abundance along road edges and within abandoned logging roads in Appalachian forests. Conservation Biology, 21, 159-67.

Shanley, C.S., \& Pyare, S. (2011). Evaluating the road-effect zone on wildlife distribution in a rural landscape. Ecological Society of America, 2, 1-16.

Simonetti, J.A. (2006). Conservacion de biodiversidad en ambientes fragmentados: el caso del bosque maulino. In A.A. Grez, J.A. Simonetti, \& R.O. Bustamante (Eds.), Biodiversidad en Ambientes Fragmentados de Chile: Patrones y Procesos a Diferentes Escalas (pp. 215-229). Santiago: Editorial Universitaria.

Smith, T.M., \& Smith, R.L. (2009). Ökologie. München: Pearson Studium Verlag.

Subdepartamento de SIG y Cartografía de la Dirección Nacional de Vialidad (GIS and Cartography Department of the Ministry of Transport). (2011). Red de Infraestructura Vial de Chile. Datos Cartográficos. Santiago: Dirección Nacional de Vialidad.

Turner, J.M. (2006). Conservation science and forest service policy for roadless areas. Conservation Biology, 20,713-722.

UBA (German Federal Environmental Agency). (2012). Daten zur Umwelt. Retrieved June 25, 2013, from http://www.umweltbundesamt-daten-zurumwelt.de/umweltdaten/public/theme.do?nodeIdent $=2858$

Unidad de Sistemas de Información Geográfica - División Regional (Department of Geographical Informationsystems - Regional Division). (2009). Red Vial - Región del Biobío y Región de la Araucaría. Santiago: Dirección de Vialidad.

Van Der Grift, E.A., Van der Ree, R., Fahrig, L., Findlay, S., Houlahan, J., Jaeger, J.A.G., Klar, N., Madriñan, L.F., \& Olson, L. (2013). Evaluating the effectiveness of road mitigation measures. Biodiversity and Conservation, 22, 425-448.

Van der Ree, R., Heinze, D., Mccarthy, M., \& Mansergh, I. (2009). Wildlife tunnel enhances viability. Ecology and Society, 14, 1-9. 



\section{ARTICLE IV}

Friedrich, J. Integrating neglected ecological impacts of road transport into corporate management. Submitted June $10^{\text {th }} 2014$ to: Ecological Indicators.

Printed with permission for non-commercial use. 



\title{
Integrating neglected ecological impacts of road transport into corporate management
}

\author{
Jan Friedrich \\ University of Göttingen, Faculty of Economics, Chair of Production and Logistics, Platz der Göttinger Sieben 3, 37073 Göttingen, Germany. \\ E-mail: jan.friedrich@wiwi.uni-goettingen.de
}

\begin{abstract}
Companies producing consumer goods are showing a greater interest in integrating the concept of 'environmental footprinting' into their management. Usually, one component of a footprint is road transport. While land use and greenhouse gas emissions are accounted for in life cycle assessment the focus often is on the latter. The additional impact by roads and vehicles - particularly regarding biological diversity - is seldom considered. One reason for this deficit is that findings from road ecology are not sufficiently quantifiable to integrate in life cycle or footprinting analyses. In this article, three approaches for quantifying direct land use, degradation of adjunct area, and fragmentation are introduced. Methodologically, this is achieved through a literature analysis and the use of case studies. Four equations are presented that allow measuring spatial impacts of transport processes. Results can be used for impact mitigation and reporting in the context of LCA.
\end{abstract}

Keywords: Biodiversity; corporate environmental management; fragmentation; indicators; land degradation; land use; life cycle assessment; impact quantification; road effect zone; road transport.

\section{Introduction}

Companies at different stages of value chains are interested in knowing the environmental impact of their products because they seek to meet consumer interests. In spite of discrete regulatory or monetary incentives like the European Emissions Trading Scheme (EU-ETS), the demands of end customers and other stakeholders are usually the strongest drivers behind a company's environmental management efforts (Christini et al. 2011). In addition, the moral dimension concerning humans' relations to other life forms and to to be born human beings (Krebs 2008, Nussbaum 2006, Becker 2010), scientific research, and governmental initiatives like the CBD increase the pressure on companies to extend their activities in the field of ecosystem service conservation (Chapin et al. 2000; Sala et al. 2000; MA 2005; CBD 2010a; CBD 2010b; CBD 2011). Due to being spatially demanding and resource and emission intensive, mobility, and here particularly road traffic, is one of the substantial causes of ecosystem degradation (Lindenmayer 2006; Andel et al. 2010). Developments in economic organization - particularly in global trade and specialization and the resulting government-support of mobility in order to assist national competitiveness are significant drivers of increments in road infrastructure and traffic volumes in the European Union member states in the last 20 years (EU 2013, p. 21\&75). For example, while the reduction of greenhouse gas emissions by route optimization, mode choice, driver training or adjusted warehouse management are in the focus of life cycle assessment (LCA) in transport, spatial ecological impacts are often neglected (Dekker et al. 2012, Murphy and Poist 2000).

The ecological impact of roads and traffic, and options to avoid and mitigate these impacts are investigated in a research field called 'road ecology'. To date, many studies of different species groups like mammals, birds, amphibians or insects as well as of different road types like forest roads or highways, have confirmed the differing but overall significantly negative impact of roads and traffic on biodiversity (Benítez-Lopez et al. 2010; Eigenbrod et al. 2009; Charry and Jones 2009; Fahrig and Rytwinski 2009). This knowledge has not reached the attention of corporate environmental management because it has not been expressed in a way where it could be used in LCA. Hence, the objective of this article is to present quantitative methods to integrate the three neglected impacts of direct land use, degradation of adjunct areas, and fragmentation into LCA and overall corporate environmental management. 
To this end, ecological findings are analyzed in a literature study in order to construct indicators that allow calculating the share of impact of an individual user. The methods presented here stop at the level of the vehicle that is used. However, if quantity, volume, and weight of freight are known, the vehicle-specific result could be itemized to determine the impact per product. This article is structured as follows: the first section describes the relevant ecological effects of road transport. Next, four approaches of quantifying the impact of land use and fragmentation are introduced. Finally, the formulae are applied to a case study. The formulae serve the purpose and procedure of LCA and can aid organizations to understand, quantify, and ultimately reduce the impact of road transport on biodiversity.

\section{Ecological Impacts of Roads and Traffic}

In this section, the impact of land use, degradation of habitat, road mortality, and fragmentation are described because the available quantitative results constitute the basis for the development of the indicators. These impacts are focused on because they are underrepresented in corporate environmental management and because their severity of impact on individuals, species and ecosystems is high.

\section{Land use and degradation of habitat}

While it may seem reasonable to group loss and degradation of habitat under the category of land use, due to the current connotation of the term land use in this text, it refers strictly to sealed area. Degraded area forms a category of its own. The soil and habitat functions of natural area can be either lost completely where surfaces are sealed; or be lost partially in areas adjunct to roads. These areas are often affected by additional artificial construction like embankments and by diffusing effects, such as noise or substance emissions. In the literature, the zone over which significant ecological effects extend outward from a road is called 'roadeffect zone' (Forman and Deblinger 2000). Besides material emissions from vehicles (such as nitrogen oxide, particulate matter, ozone, carbon monoxide, carbon dioxide, sulfur dioxide, non-methane hydrocarbons, rubber or litter) and from the road (salt, dust), intangible emissions like vibration, heat, noise, and light occur (Friedrich and Geldermann 2013b). Furthermore, a road and the physical modification of the landscape can produce changes in local climate because winds, temperature, and moisture can change. In addition, hydrologic systems can be altered and corresponding downstream effects occur because roads may block water flows, increase the risk of flooding, and contribute to erosion and subsequent sedimentation (Forman et al. 2003).

Studies have found outward effects with significant impact extending from about 15 to 200 meters for material emissions, which can repress the germination of plants. These outward effects can also change competitive relations and hence, plant composition. Vibrations and noise extend between 200 to 800 meters, affecting tortoise, anuran species, or breeding-birds and their breeding success (Eigenbrod et al. 2009; Boarman and Sazaki 2004; Reijnen and Foppen 1994; Reijnen et al. 1997). Impact can occur for up to 2,000 to 5,000 meters for distinct mammalian species that are extremely sensitive to noise, such as female moose (Reck and Kaule 1992; Kaseloo 2006; Shanley and Pyare 2011; Benítez-López et al. 2010). A non-speciesspecific study found that $33 \%$ of the significant impacts lie within the first 100 meters, while only $8 \%$ of the impact occurs further than 500 meters away from the road (Biglin and Dupigny-Giroux 2006).

For a highway with 50,000 vehicles per day, Forman and Deblinger (2000) estimated the affected zone to average 600 meters in width. The width of a road-effect zone predominantly depends on traffic volume and on the topography of a landscape (Shanley and Pyare 2011; Charry and Jones 2009). Regarding the aggregate landscape-level impact, Forman (2000) calculated that while roads in the U.S. cover $1 \%$ of the land, they essentially affect $20 \%$ of the total land mass. For Germany, in $201215,318 \mathrm{~km}^{2}$ or $4.29 \%$ of the surface was sealed by motorized road-bound infrastructure, affecting more than $50 \%$ of the country's landscape (Federal Statistical Office 2013; calculations extended by the authors). With regard to sealed area the indicator to be introduced advances the existing methodology because it moves from considering national total area sealed by roads and vehicle type to considering average sealed area per road type, road size per lane quantity, average intensity of use, and vehicle type. Next, the impact of road mortality is referred to, because besides often being the first perceived problem by humans and the most devastating impact at the level of an individual, it can build to be one reason of fragmentation.

\section{Road mortality}

Animals need to move for instance to reach food, resting places, mates or seasonal habitats. Collisions in roads are not limited to usually being fatal for individuals, but for collision-prone species also pose a 
significant problem at the population-level. In Germany, road mortality was the most probable cause of death for otters and wild cats in 2006 (Herrmann et al. 2007). In North America, the annual number of animalvehicle collisions is estimated to be 1-2 million (references in Huijser et al. 2009). Attempts have been made to reduce animal mortality by mitigation measures such as wildlife fences, separation barriers, speed limits, warning signs, and wildlife crossing structures (van der Grift et al. 2013). While road width and vehicle speed influence the degree of risk, various studies identify traffic volume as the dominant factor in unsuccessful crossings (Jaeger et al. 2005a; Charry and Jones 2009). Studies specifically investigating traffic volumes suggest that roadkill rates increase as traffic increases up to a level of about 3,000 vehicles per day. For traffic levels between 3,000 and 10,000 vehicles, the rate remains high or may begin to drop and will drop further for levels above 10,000 vehicles as the density of vehicles and noise deters animals from crossings (Charry and Jones 2009; Forman and Sperling 2011). This has a partly positive effect because it means that the effect of the road as a barrier is now substantial. Despite the decline in average roadkill rates as daily traffic volume declines, the total effect of minor roads is still significant due to their large presence in the landscape (Taylor and Goldingay 2010). Overall, population size, species occurrence, velocity of animal movement, species' diurnal movement patterns and the number and characteristics of vehicles are the most important factors influencing species' vulnerability to road mortality (Hels and Buchwald 2000; Riley et al. 2006). In this paper, no separate indicator measuring an individual vehicle's impact of road mortality is presented because the impact is landscape-, site- and species-specific. However, it is accounted for in part in the indicators of fragmentation because mortality contributes to the impact of fragmentation for roads with daily traffic volumes between 500 and 10,000 vehicles.

\section{Habitat fragmentation}

Landscape fragmentation is the result of transforming large habitat patches into smaller, more isolated fragments of habitat (EEA 2011). The phenomenon consists of two slightly different effects: the subdivision of populations, and the barrier effect, meaning that animals and plants are unable to move freely within a landscape. A road can represent a barrier in three principal ways:

1) Animals can be deterred from approaching a road because of noise, light, or changes in habitat. When they do come to a road, they can be deterred from crossing the road because of physical obstacles like fences, median barriers, or even the road surface, which may display uninviting conditions for being dry or for being without shelter; or because of the presence of fast moving vehicles, which increase levels of noise and stress. This applies to life on the ground, in the air, and in streams.

2) Animals that attempt to cross a road may die. Death is predominantly of the result of collision with vehicles, but can also be attributed to attack by predators.

3) Spores, insects, and other life forms that do not actively cross a road but are carried over by host animals, can no longer disperse if one of the before mentioned aspects applies to their host.

The barrier effect is problematic because it impedes an individual animal's access to resources like food or shelter, or mates (Bertiller et al. 2007). Consequences from denied access to mates can be inbreeding and decreased variability of genomes (Keller and Largiader 2003; Fischer and Matthies 1998; Riley et al. 2006). The risk of genetic differentiation and extinction due to the barrier effect is significantly higher in species with low dispersal abilities (Keller and Largiadèr 2004), in specialist species that require niche habitats (Fahrig 2003) and in mammals with large habitat demands (Yahner 1988; Herrmann et al. 2007). The effect of genetic subdivision is particularly dangerous because the time lag between the occurrence of an impact and the full ecological consequences can take decades and to date are sparely understood (Tilman et al. 1994; Jaeger et al. 2005b). More information needs to be generated by applying molecular or genetic approaches to a higher degree (Balkenhol and Waits 2009). This type of investigation is particularly needed to study the effectiveness of wildlife crossing structures, which seem to become an increasingly popular strategy of mitigation (Corlatti et al. 2009; van der Grift et al. 2013). A slightly different effect of landscape fragmentation is the subdivision of a meta-population or a smaller population. The four negative effects that subpopulations then face are: the edge effect, which means that the share of less favorable area increases, demographic instability, which means an increased risk of a gender ratio unfavorable for reproduction, a greater thread of changing environmental factors, and diminished genetic variability (Oggier et al. 2007).

In Germany, fragmentation is not measured by responses in animals or plants but is measured at a landscape level. The main approach that is used is the calculation of the percentage of lowly-disturbed areas larger than $100 \mathrm{~km}^{2}$ (see Schupp 2005 and Lassen 1987). Having a weakness in falling short of referring to the ecological quality of the areas designated as valuable due to their large size, advantages of the approach are its intuitive understanding and the assumption that an undissected area of a certain size is both a prerequisite and a facilitator for the functioning of regional, national and supranational ecosystems and their 
species diversity (Turner 2006; Selva et al. 2011; Baier and Holz 2001). Having fallen from 26.5\% in 2000 to $25.4 \%$ in 2005 to $23.16 \%$ in 2010 , Germany's 'National Strategy on Biological Diversity' aims to maintain low traffic areas larger than $100 \mathrm{~km}^{2}$ to at least 23\% (BMU 2010; BMU 2011; BfN 2013). While this quantitative data was regarded too generic to be used for the measurement of individual vehicle impact, a study that determined and weighted over 10,000 locations of fragmenting conflict in Germany for species of four different habitats was decided to be employed (Hänel and Reck 2011). As the goal of the study was to identify the most severe locations of conflict in order to mitigate most effectively - and not to facilitate the quantification of fragmenting impact of an arbitrary route - data was not processed in a way that can be applied to an algorithm, but has to be extracted from habitat-specific maps.

Other negative impacts related to road transport that shall briefly be mentioned are the spread of invasive alien species, erosion and sedimentation, changes in hydrologic systems, emission of greenhouse gases, and the effects occurring along the entire life cycle of both vehicles and road (Friedrich and Geldermann 2013a; Forman and Sperling 2011). While road transport also has positive effects on the natural environment, an empirical review of 79 studies found that the negative effects outnumber the positive effects by a factor of five (Fahrig and Rytwinski 2009). In the next section, it is explained how the developed indicators employ the information referred to above.

\section{Development of Quantitative Indicators for Land Use and Fragmentation}

From a review of the existing literature, enough evidence on the characteristics of the impacts referred to above could be derived in order to build four indicators that allow measuring the share of land use, of degraded area, and of fragmentation of an individual transport process. In the following section, the reasoning and the required variables of the formulae are specified. The formula that quantifies the share of sealed area of an individual vehicle yields particularly precise results for traffic volumes above 5,000 vehicles on federal highways and freeways. This is because of good data availability, homogeneity, and an analytical emphasis of high-use roads. In order to obtain dependable results for the share of sealed area, the percentage of roads that are of a lower road type than federal highways, or that carry less than 5,000 vehicles per day should be below $5 \%$ of an entire route. For the three remaining formulae that concern the impacts of habitat degradation and fragmentation, results are reliable for traffic volumes as low as about 1,000 vehicles per day. Here, for reliable results, the share of roads with less than 1,000 vehicles per day should not surpass $5 \%$ of an entire route.

The approach to determine the share of sealed area (SSA) of a vehicle is to calculate the area covered by the route and to divide this number by the quantity of users within one year. Sealed areas are split amongst all users for one year because this time horizon represents a compromise between a road to be in place usually for several years and the impact of sealed soil to be effective every day. Also, yearly results are suitable for companies because their reporting and management periods are usually for one year. While the distance of a trip is easily known, road width is more difficult to assess. Instead of using the gross national area covered by roads, in this approach, actual road width is accounted for. Road width is approximated per quantity of lanes per type of road. Average lane widths, which were determined to be $7.48 \mathrm{~m}$ for freeways (FW), $4.96 \mathrm{~m}$ for federal highways (FH) and $4 \mathrm{~m}$ for smaller roads (SR), account for the area sealed by the entire construction of a road and by bypasses and resting areas of the particular road type. Also, the average number of users usually varies within a route, which is why the method divides a route into individual sections, the results of which are to be added.

The variables that divide a route into sections whenever one of them changes are average daily traffic volume $\left(\mathrm{dtv}_{\mathrm{i}}\right)$, road type $(\mathrm{FW}, \mathrm{FH}, \mathrm{SR})$ and the number of lanes $\left(\mathrm{l}_{\mathrm{i}}\right)$. Resulting lengths of sections $\left(\mathrm{d}_{\mathrm{i}}\right)$ are multiplied by the average lane width for the road type and by the quantity of lanes of the road. The intermediary result is divided by the total impact of all users over the period of one year. In the last step, the impact factor $\left(f\left(v_{x}\right)\right)$ that differs by vehicle size is accounted for by the means of passenger car units (PCUs). Recognizing the vehicle factors of all users, no site-specific approach is applied, but shares of national mileage are assumed yielding a total of 1.1379. The result quantifies the share of sealed area that an individual vehicle is responsible for. The unit of measurement of the result is square meters. The equation suggested for calculation is indicated below. Assumed vehicle factors are specified in Table 1.

$$
S S A=\sum_{i=1}^{n} \frac{d_{i F W} \cdot 7.48 m \cdot l_{i}+d_{i F H} \cdot 4.96 m \cdot l_{i}+d_{i S R} \cdot 4 m \cdot l_{i}}{d t v_{i} \cdot(1.1379) \cdot 365} \cdot f\left(v_{x}\right)
$$


Table 1: Mileage shares and vehicle factors; adapted from Elsner 2010, Delft et al. 2008, ProgTrans AG and IWW 2007.

\begin{tabular}{|c|c|c|c|}
\hline Vehicle Type $\left(\mathbf{v}_{\mathbf{x}}\right)$ & $\begin{array}{l}\text { National Mileage } \\
\text { in billion tkm }\end{array}$ & $\begin{array}{c}\text { National } \\
\text { Mileage in \% }\end{array}$ & $\begin{array}{c}\text { Vehicle Factor/ } \\
\operatorname{PCU} \mathbf{f}\left(\mathbf{v}_{\mathbf{x}}\right)\end{array}$ \\
\hline Motorcycles $\left(\mathrm{v}_{\mathrm{m}}\right)$ & 15.4 & 2.23 & $0.5 \mathrm{f}\left(\mathrm{v}_{\mathrm{m}}\right)$ \\
\hline Cars \& Combination Vehicles $\left(\mathrm{v}_{\mathrm{c}}\right)$ & 587.5 & 84.90 & $1.0 \mathrm{f}\left(\mathrm{v}_{\mathrm{c}}\right)$ \\
\hline Buses $\left(v_{b}\right)$ & 3.4 & 0.49 & $2.5 \mathrm{f}\left(\mathrm{v}_{\mathrm{b}}\right)$ \\
\hline Pick-up Trucks $\leq 3.5$ tons $\left(\mathrm{v}_{\mathrm{t} 3.5}\right)$ & & 5.04 & $1.2 \mathrm{f}\left(\mathrm{v}_{\mathrm{t} 3.5}\right)$ \\
\hline Trucks $>3.5 \leq 12$ tons $\left(\mathrm{v}_{\mathrm{t} 12}\right)$ & & 1.67 & $2.0 \mathrm{f}\left(\mathrm{v}_{\mathrm{t} 12}\right)$ \\
\hline Trucks $>12$ tons $\left(\mathrm{v}_{\mathrm{t}}>12\right)$ & & 2.08 & $3.0 \mathrm{f}\left(\mathrm{v}_{\mathrm{t}>12}\right)$ \\
\hline All trucks combined & 60.8 & 8.79 & \\
\hline Semitrailer Tractors $\left(\mathrm{v}_{\mathrm{st}}\right)$ & 16.9 & 2.44 & $4.0 \mathrm{f}\left(\mathrm{v}_{\mathrm{st}}\right)$ \\
\hline other $\left(v_{0}\right)$ & 8.0 & 1.16 & $1.0 \mathrm{f}\left(\mathrm{v}_{\mathrm{o}}\right)$ \\
\hline Total & 692.0 & 100.01 & \\
\hline
\end{tabular}

The degrading impact on adjunct area consists of various individual impacts like tangible and intangible emissions, invasive species, erosion or changes in hydrology. In order to construct a formula to quantify the total impact, the impact of noise is focused on because considering both reach and immediacy of impact, it is the spatially most significant one. A sound pressure level of $55 \mathrm{~dB}(\mathrm{~A})$ is set as the lowermost threshold for disturbing impact. During the research, firstly, a theoretical approach that relates laws and formulae used for the calculation of noise zone extensions by governmental institutions was followed. Next, a practical approach that builds a formula from a data sample of mapped noise zones of roads in Germany was applied. While results were comparable, the latter approach was regarded more reliable. The formula that is best suited to represent the data sample is noise zone width $=0,2212 \cdot d t v_{i}{ }^{0.7273}$. It expresses the correlation of generated sound pressure level and traffic volume. To calculate the dissimilar reaches of noise levels $\geq 55$ $\mathrm{dB}(\mathrm{A})$ again average daily traffic volume $\left(\mathrm{dtv}_{\mathrm{i}}\right)$ partitions a route into individual sections, the lengths of which $\left(\mathrm{d}_{\mathrm{i}}\right)$ constitute for the second necessary variable. The impact also being vehicle-specific, vehicle factor $\left(f\left(v_{x}\right)\right)$ makes for the third variable. However, vehicle factors were found to differ from those used for the impact of sealed area. They are indicated in Table 2. The resulting formula of quantification is entitled NDLI (noise driven landscape impact). NDLI quantifies the extending habitat degrading impact of an individual road user (see the below equation). The unit of measurement of the result is square meters.

$$
N D L I=\sum_{i=1}^{n}\left(\frac{0,2212 \cdot d t v_{i}^{0.7273} \cdot d_{i} \cdot 2}{d t v_{i} \cdot(1.3544) \cdot 365}\right) \cdot f\left(v_{x}\right)
$$

Table 2: Mileage shares and vehicle factors; mileages adapted from Elsner 2010.

\begin{tabular}{|c|c|c|c|}
\hline Vehicle Type $\left(\mathbf{v}_{x}\right)$ & $\begin{array}{l}\text { National Mileage } \\
\text { in billion tkm. }\end{array}$ & $\begin{array}{c}\text { National } \\
\text { Mileage in \% }\end{array}$ & $\begin{array}{c}\text { Vehicle Factor/ } \\
\operatorname{PCU} \mathbf{f}\left(\mathbf{v}_{\mathbf{x}}\right)\end{array}$ \\
\hline Motorcycles $\left(\mathrm{v}_{\mathrm{m}}\right)$ & 15.4 & 2.23 & $1.0 \mathrm{f}\left(\mathrm{v}_{\mathrm{m}}\right)$ \\
\hline Cars \& Combination Vehicles $\left(\mathrm{v}_{\mathrm{c}}\right)$ & 587.5 & 84.90 & $1.0 \mathrm{f}\left(\mathrm{v}_{\mathrm{c}}\right)$ \\
\hline Buses $\left(\mathrm{v}_{\mathrm{b}}\right)$ & 3.4 & 0.49 & $2.5 \mathrm{f}\left(\mathrm{v}_{\mathrm{b}}\right)$ \\
\hline Pick-up Trucks $\leq 3.5$ tons $\left(\mathrm{v}_{\mathrm{t} 3.5}\right)$ & & 5.04 & $2.5 \mathrm{f}\left(\mathrm{v}_{\mathrm{t} 3.5}\right)$ \\
\hline Trucks $>3.5 \leq 12$ tons $\left(\mathrm{v}_{\mathrm{t} 12}\right)$ & & 1.67 & $3.5 \mathrm{f}\left(\mathrm{v}_{\mathrm{t} 12}\right)$ \\
\hline Trucks $>12$ tons $\left(\mathrm{v}_{\mathrm{t}>12}\right)$ & & 2.08 & $5.0 \mathrm{f}\left(\mathrm{v}_{\mathrm{t}>12}\right)$ \\
\hline All trucks combined & 60.8 & 8.79 & \\
\hline Semitrailer Tractors $\left(\mathrm{v}_{\mathrm{st}}\right)$ & 16.9 & 2.44 & $7.0 \mathrm{f}\left(\mathrm{v}_{\mathrm{st}}\right)$ \\
\hline other $\left(v_{o}\right)$ & 8.0 & 1.16 & $1.0 \mathrm{f}\left(\mathrm{v}_{\mathrm{o}}\right)$ \\
\hline Total & 692.0 & 100.01 & \\
\hline
\end{tabular}

As mentioned above, the impact of fragmentation is difficult to ascertain for many reasons. These reasons include the fact that fragmentation concerns many species differently; the character and the consequences of the impact are not fully understood; and the valuation and understanding human-/ nature-relations, biodiversity goals, and willingness to carry risk. Thus, the impact is expressed as 'fragmenting value' (FV) and two indicators representing philosophies from both ends of the spectrum of valuation are introduced. 
Their use is not a question of either/ or, because they are viewed to complement each other. While one approach is more anthropogenic and mitigation-centered, the other does not discern species-importance or quantity of concerned parties and is avoidance-oriented. The first description refers to the corridor and habitat oriented approach (FVcha).

To attempt expressing the fragmenting impact of a vehicle driving a certain route, under FVcha, the before mentioned study - that identified and prioritized conflicts between traffic infrastructure and movement corridors of species of four different habitat types, wet, dry, forest, and forest living large mammal habitats - is used. Weighting of the conflicts depends on the ecological importance of a habitat or corridor, on the severity of the barrier function of an infrastructure, and on the presence or absence of opportunities for wildlife to cross a barrier. In the method proposed below, conflict points and conflict values are taken from the final maps of the respective study (Hänel et al. 2010). In order to account for the severity of the conflicts, which is described by assigning conflicts to conflict groups 1 through 6 for each habitat type $\left(\mathrm{cg}_{1} \mathrm{~h}, \ldots, \mathrm{cg}_{6} \mathrm{~h}\right)$, group totals $\left(x_{c g_{i} h}\right)$ are multiplied by numeric values ranging from 5 to 0.25 , where 5 concerns the group of severest conflicts $\left(\mathrm{cg}_{1} \mathrm{~h}\right)$ and 0.25 the group of least severe conflicts $\left(\mathrm{cg}_{6} \mathrm{~h}\right)$. The intermediate result is to be multiplied by a vehicle factor $\left(\mathrm{f}\left(\mathrm{v}_{\mathrm{x}}\right)\right)$, which in this case is the same as in the calculation of the share of sealed area. The resulting equation is indicated below.

$$
F V c h a=\sum_{h=w, d, f, l m}\left(x_{c g_{1} h} \cdot 5+x_{c g_{2} h} \cdot 4+x_{c_{g 3} h} \cdot 3+x_{c g_{4} h} \cdot 2+x_{c_{5} h} \cdot 1+x_{c g_{6} h} \cdot 0.25\right) \cdot f\left(v_{x}\right)
$$

The second approach suggested to measure fragmenting impact complements the result of FVcha because it applies a discretionary landscape-oriented assessment of impact, meaning that all locations of the landscape are treated as equally important. The argument for this valuation is that roads and traffic in most cases impede exchanges of biotic and abiotic matter. Also, due to incomplete knowledge of the importance of those interchanges, and the discrimination of the individual when relying on formulations of priority stemming from biodiversity-, greatest-number- or visibility-aspects, it is appropriate to carry out a discretionary approach. A fragmenting impact can also be produced by smaller and lower use roads or even by decommissioned gravel roads for some amphibian species (Semlitsch et al. 2007). Yet the dynamic that higher use roads usually represent an impact to a larger number of animals is used by the approach of quantification because it derives the strength of fragmenting impact from daily traffic volume. Literature on average severities of fragmentation at various traffic volumes yields the two functions $f\left(\mathrm{dtv}_{\mathrm{i}}\right)$ for traffic volumes either above or below 5,000 vehicles per day. The equations are indicated above the fraction line of the FVdla-formula depicted below. As in the two indicators concerning land use and degraded area, FVcha calculates the share of impact of an individual user. Hence, average daily traffic volume partitions a route into individual sections, the results of which are added and adjusted by the vehicle factors in Table 1 to obtain the final result. Besides $\mathrm{dtv}_{\mathrm{i}}$, the distance traveled in each section $\left(\mathrm{d}_{\mathrm{i}}\right)$ and the vehicle factor $\left(\mathrm{f}\left(\mathrm{v}_{\mathrm{x}}\right)\right)$ are the variables that need to be known.

$$
\text { FVdla }=\sum_{i=1}^{n} \frac{d_{i} \cdot f\left(d t v_{i}\right)=\left\{\begin{array}{c}
2,8498 \ln \left(d t v_{i}\right)-21,317 \quad \text { for all } d t v[\geq 5,000] \\
0.009816 d t v_{i}^{0,6701} \quad \text { for all } 500<d t v<5,000
\end{array}\right\}}{d t v_{i} \cdot(1.1379)} \cdot f\left(v_{x}\right)
$$

The formulae have the following weaknesses. Calculating the share of sealed area, the method can be applied to less used roads, but the accuracy of results is expected to decline. Regarding the formula developed to determine the extension of degraded area, the consideration of noise as a single impact to account for other extending ecological impacts and the assumption of a noise disturbance threshold at $55 \mathrm{~dB}(\mathrm{~A})$ appear to require further discussion. Regarding the quantification of fragmenting impact of an individual vehicle, the assumed weighting of the different priorities of conflict when following a corridor and habitat-oriented approach, and the inclusion of urban area under the discretionary landscape approach are considered potential limitations. Two constraints that concern all four indicators are the set vehicle factors, which have not been applied to environmental contexts, and the missing compilation of all necessary data in one database. Regarding the applicability of the approaches, the two formulae measuring degradation of adjunct area and fragmenting impact when pursuing a discretionary landscape approach have universal validity. The 
formulae measuring sealed area and fragmenting impact when applying a corridor and habitat approach hold limitations in precision and applicability because they depend on data specific to Germany. Even with the limitations, the methodologies of all approaches can be applied globally.

\section{Exemplary Application of the Methods of Quantification}

In order to present possible results and to illustrate the characteristics of the indicators, the formulae are applied exemplarily. Below the impacts of two vehicles driving two different routes are quantified. One is a $355 \mathrm{~km}$ long round-trip within the German State of Lower Saxony leading from Hann. Münden to Hannover to Brunswick and back to Hann. Münden. The second route goes from Northern to Southern Germany, from Hannover to Wendelstein in Bavaria. The distance of the route is $480 \mathrm{~km}$. The frequency of the first transport is 40 trips per year, the second is assumed to take place 1.4 times a week on average totaling 73 trips per year. In both cases, semitrailer tractors are used. Results are indicated below.

Table 3: Results of the individual impact of route 1; distance of the trip: $354.5 \mathrm{~km}$; frequency of the trip: 40 trips per year.

\begin{tabular}{|c|c|c|c|}
\hline Type of impact & ...per trip & $\begin{array}{l}\text { Impact ... } \\
\text {...per year }\end{array}$ & $\ldots$ per $1 \mathrm{~km}$ \\
\hline SSA (in $\mathrm{m}^{2}$ ) & 2.92 & 116.72 & 0.0082 \\
\hline NDLI (in $\mathrm{m}^{2}$ ) & 126.82 & $5,072.77$ & 0.3577 \\
\hline FVcha & 302.00 & $12,080.00$ & 0.8519 \\
\hline FVdla & 293.80 & $11,752.03$ & 0.8288 \\
\hline
\end{tabular}

Table 4: Results of the individual impact of route 2; distance of the trip: $479.9 \mathrm{~km}$; frequency of the trip: 73 trips per year.

\begin{tabular}{|c|c|c|c|}
\hline Type of impact & ... per trip & $\begin{array}{l}\text { Impact ... } \\
\text {... per year }\end{array}$ & $\ldots$ per $1 \mathrm{~km}$ \\
\hline SSA (in $\left.\mathrm{m}^{2}\right)$ & 3.09 & 225.80 & 0.0064 \\
\hline NDLI (in $\left.\mathrm{m}^{2}\right)$ & 156.49 & $11,423.96$ & 0.3261 \\
\hline FVcha & 533.00 & $38,909.00$ & 1.1106 \\
\hline FVdla & 318.11 & $23,222.18$ & 0.6629 \\
\hline
\end{tabular}

Legend: $\mathrm{SSA}=$ share of sealed area; NDLI $=$ noise driven landscape impact $; \mathrm{FV}$ cha $=$ fragmenting value using a corridor and habitat orientated approach; FVdla = fragmenting value using a discretionary landscape orientated approach.

Results of $2.92 \mathrm{~m}^{2}$ for route 1 and $3.09 \mathrm{~m}^{2}$ for route 2 as values of sealed area and of $127 \mathrm{~m}^{2}$ for route 1 and $156 \mathrm{~m}^{2}$ for route 2 for portions of degraded area appear to be reliable. When comparing the results of the two routes by assessing the kilometric results, apart from the result for fragmenting value of the corridor and habitat oriented approach, route 2 yields ecologically superior numbers. For the share of sealed area and the fragmenting value under the discretionary landscape approach, the difference in results per kilometer explains because route 2 uses less smaller roads, the percentage being $0.85 \%$ compared to $4.24 \%$ for route 1 , and because overall average traffic intensity was higher on route 2 with 54,416 vehicles per kilometer compared to 50,502 vehicles per kilometer for route 1 . The greater efficiency of high use roads also becomes evident in a superior kilometric value for degraded area. The finding that the difference is more moderate indicates the comparatively linear relation between traffic volume and extending impact. The reason that route 2 yields a higher result of fragmenting value using a corridor and habitat-oriented approach is that it runs through less build up area and hence, has a higher probability of producing fragmenting conflict compared to route 1.

Testing both routes for potentially superior courses, the fact that in both cases, more frequently used roads were selected over less frequently used alternatives produced the result that no alternative courses were found that would improve the above mentioned results. This is favorable because it underlines that the formulae function in favor of the general opinion that concentrating traffic on fewer, higher use roads is environmentally preferable to spreading traffic more evenly across the landscape. Companies can use the results of the indicators not only to select a certain route. By knowing either quantity or weight of the freight, 
the quantified impacts can be broken down and be apportioned to products. Regarding an entire company, relating total impacts of land use and fragmentation to inflation-adjusted sales or calculating results for a functional unit can facilitate impact identification and management.

\section{Conclusion}

Information emerging from natural sciences and increasing environmental awareness amongst stakeholders indicate that organizations in the future will seek to offer products of with low environmental impact. To assist reaching this objective, this paper outlines four approaches of quantifying neglected ecological impacts. Here, the relevance of the ecological effects of land use, degradation of adjunct area, and fragmentation produced by road haulage were explained and exploited in order to support their inclusion into ecobalancing and corporate environmental management. The quantitative results of the indicators can be reported and used to improve the management of their mitigation. One strength of the formulae is that apart from reducing haulage distances, a prioritization for using larger roads brings down results effectively. Regarding fragmentation, this course of action matches current strategies of defragmentation, which favor efforts towards bundling traffic and to then apply solutions that ensure landscape connectivity across the resulting fewer roads (Jaarsma and Willems 2002; Charry and Jones 2009; EEA 2011).

While the employment of the indicators may be the task of environmental management departments, the discussion, elaboration and furthermost execution of strategies of avoidance and mitigation concern various functional units of an organization. Reducing total haulage distance per unit in sourcing and delivering, and using spatially more efficient and frequently-used roads, from a corporate perspective are the two most promising courses of action to diminish the effects of land use and fragmentation. However, despite the relevance of this research to contribute to more complete environmental management and allocation (including consumer-impact), the indicators introduced above shed only a small light on answering greater environmental issues faced by industrialized and industrializing countries' societies. Individual ethical reflections of human's three fundamental relationships with contemporaries, past and future generations, and non-human nature, which at the level of consumptive action all deal with the question of how to share what a finite planet offers, as well as reflections of the quality of currently dominating economic and political organization and agenda to support all humans to live dignified and flourishing lives appear to be of greater importance. As far as degrees of sharing with non-human nature, which would help maintain abundance and diversity of other life forms, are concerned, a renunciation of material growth and a parallel adoption and organization of less ecologically demanding lifestyles, possibly recognizing complementary attitudes like simplicity as virtues of the good life, seem necessary.

ACKNOWLEDGEMENTS: I acknowledge the support of my colleagues from the interdisciplinary PhD program "Biodiversity and Society" and the help of my co-workers from the Chair of Production and Logistics at Göttingen University.

\section{REFERENCES}

Andel, P., T. Minarikova, and M. Andreas. 2010. Protection of landscape connectivity for large mammals . Liberec: Everina.

Baier, H., and R. Holz. 2001. Landschaftszerschneidung als Naturschutzproblem: Die Wirkungen und ihre Vermeidungsstrategien. Naturschutzarbeit in Mecklenburg-Vorpommern 44, 11-27.

Balkenhol, N., and L.P. Waits. 2009. Molecular road ecology: exploring the potential of genetics for investigating transportation impacts on wildlife. Molecular Ecology 18: 4151-4164.

Baumert, K.A., T. Herzog, and J. Pershing. 2005. Navigating the Numbers. Greenhouse Gas Data and International Climate Policy. Washington, DC: World Resource Institute.

Becker, Ch. 2010. Sustainability ethics , Pennsylvania: STS Program and Department of Philosophy, Pennsylvania State University.

Benítez-López, A., R. Alkemade, and P.A. Verweij. 2010. The impacts of roads and other infrastructure on mammal and bird populations: a meta-analysis. Biological Conservation 143: 1307-1316. 
Bertiller, R., C. Schwick, and J.A.G. Jaeger. 2007. Landschaftszerschneidung Schweiz. Zerschneidungsanalyse 1885 - 2002 und Folgerungen für die Verkehrs- und Raumplanung . Bern: ASTRABericht.

BfN (Federal Agency for Nature Conservation). 2013. Ermittlung der Unzerschnittenen Verkehrsarmen Räume > $100 \mathrm{~km}^{2}$ für Deutschland 2010 . Leipzig: BfN.

Biglin, K., and L.-A. Dupigny-Giroux. 2006. Mapping the road-effect zone to assess impacts of proposed road segments. Journal of Conservation Planning 2: 1-16.

BMU (Federal Ministry for the Environment, Nature Conservation and Nuclear Safety). 2011. National strategy on biological diversity . $3^{\text {rd }}$ edition. Berlin: BMU.

BMU (Federal Ministry for the Environment, Nature Conservation and Nuclear Safety). 2010. Der Indikatorenbericht 2010 zur Nationalen Strategie zur biologischen Vielfalt . Eichenau: Schottenheim druck\&werbung.

BMU (Federal Ministry for the Environment, Nature Conservation and Nuclear Safety). 1998. Mit dem Entwurf eines umweltpolitischen Schwerpunktprogrammes neue Etappe in der Umweltpolitik eingeleitet . Bonn: BMU-Pressedienst.

Boarman, W.I., and M. Sazaki. 2006. A highway's road-effect zone for desert tortoises (Gopherus agassizii). Journal of Arid Environments 65: 94-101.

CBD (Convention on Biological Diversity). 1992. Text of the Convention on Biological Diversity. Http://www.cbd.int/convention/text/ (accessed 5 February 2013).

CBD (Convention on Biological Diversity). 2010a. Biodiversity Scenarios: Projections of 21st Century Change in Biodiversity and Associated Ecosystem Services . Montreal: Secretariat of the Convention on Biological Diversity.

CBD (Convention on Biological Diversity). 2010b. Global Biodiversity Outlook 3 . Montreal: Secretariat of the Convention on Biological Diversity.

CBD (Convention on Biological Diversity). 2011. Development of National Biodiversity Targets in the framework of the Aichi Biodiversity Targets. Https://www.cbd.int/nbsap/about/targets/ (accessed 29 January 2013).

Chapin III, F.S., E.S. Zavaleta, V.T. Eviner, R.L. Naylor, P.M. Vitousek, H.L. Reynolds, D.U. Hooper, S. Lavorel, O.E. Sala, S.E. Hobbie, M.C. Mack, and S. Díaz. 2000. Consequences of changing biodiversity. Nature 405: 234-242.

Charry, B., and J. Jones. 2009. Traffic volume as a primary road characteristic impacting wildlife: a tool for land use and transportation planning. In Proceedings of the international conference on ecology and transportation, eds. P.J. Wagner, D. Nelson and E. Murray, pp. 159-172. Raleigh: Center for Transportation and the Environment.

Christini, G., D.H. Matthews, and C.T. Hendrickson. 2011. Corporate Environmental Management. Http://gdi.ce.cmu.edu/gd/Research/ems-03.pdf (accessed 28 January 2013).

Corlatti, L., K. Hackländer, and F. Frey-Roos. 2009. Ability of wildlife overpasses to provide connectivity and prevent genetic isolation. Conservation Biology 23: 548-556.

Dekker, R., J. Bloemhof, and I. Mallidis. 2012. Operations Research for green logistics - An overview of aspects, issues, contributions and challenges. European Journal of Operational Research 219: 671-679.

EEA (European Environmental Agency). 2011. Landscape fragmentation in Europe - Joint EEAFOEN report . Copenhagen: EEA.

Eigenbrod, F., St.J. Hecnar, and L. Fahrig. 2009. Quantifying the road-effect zone: threshold effects of a motorway on anuran populations in Ontario, Canada. Ecology and Society 14: 1-18.

EU (European Union). 2013. EU transport in figures. Statistical Pocketbook 2013. Http://ec.europa.eu/transport/facts-fundings/statistics/doc/2013/pocketbook2013.pdf (accessed 12 March 2014). 
Fahrig, L. 2003. Effects of habitat fragmentation on biodiversity. Annual Review of Ecology, Evolution, and Systematics 34: 487-515.

Fahrig, L., and T. Rytwinski. 2009. Effects of roads on animal abundance: an empirical review and synthesis. Ecology and Society 21: 1-20.

Federal Statistical Office. 2011. Surface area according to actual use, 2010 (Bodenfläche nach Art der tatsächlichen Nutzung, 2010). Wiesbaden: Federal Statistical Office.

Federal Statistical Office. 2013. Surface area according to actual use, 2012 (Bodenfläche nach Art der tatsächlichen Nutzung, 2012). Wiesbaden: Federal Statistical Office.

Fischer, M., and D. Matthies. 1998. RAPD variation in relation to population size and plant fitness in the rare Gentianella germanica (Gentianaceae). American Journal of Botany 85: 811-819.

Forman, R.T.T., and R.D. Deblinger. 2000. Ecological Road-Effect Zone of a Massachusetts Suburban Highway. Conservation Biology 14: 36-46.

Forman, R.T.T. 2000. Estimate of the area affected ecologically by the road system in the United States. Conservation Biology 14: 31-35.

Forman, R.T.T., D. Sperling, J.A. Bissonette, A.P. Clevenger, C.D. Cutshall, V.D. Dale, L. Fahrig, R. France, C.R. Goldman, K. Heanue, J.A. Jones, F.J. Swanson, T. Turrentine, and T.C. Winter. 2003. Road Ecology: Science and Solutions. Washington, DC: Island Press.

Forman, R.T.T., and D. Sperling. 2011. The future of roads: No driving, no emissions, nature reconnected. Solutions 5: 10-23.

Friedrich, J., and J. Geldermann, J. 2013a. Potentielle Auswirkungen von Straßenverkehrstransporten auf die Biodiversität. In Treffpunkt Biologische Vielfalt 12: Interdisziplinärer Forschungsaustausch im Rahmen des Übereinkommens über die Biologische Vielfalt, eds. U. Feit and H. Korn, pp. 107-113. Bonn: Federal Agency for Nature Conservation.

Friedrich, J., and J. Geldermann. 2013b. Current practices of mitigating the ecologically harmful effects of roads - An assessment. In Societal dimensions of the conservation and utilization of biological diversity, eds. J. Friedrich, A. Halsband and L. Minkmar, pp. 341-358. Goettingen: Goettingen University Press.

Taylor, B.D., and R.L. Goldingay. 2010. Roads and wildlife: impacts, mitigation and implications for wildlife management in Australia. Wildlife Research 37: 320-331.

Hänel, K., H. Reck, M. Böttcher, M. West, A. Huckauf, J. Jessberger, R. André, M. Herrmann, N. Klar, U. Walz, L. Stratmann, and J. Schumacher. 2010. Prioritäten zur Wiedervernetzung von Lebensraumkorridoren im überregionalen Straßennetz - Netzwerk für Wald bewohnende, große Säugetiere. Http://bfn.de/fileadmin/MDB/documents/themen/eingriffsregelung/2012_Prioritaeten_Gro\%DFsaeuger_Lay out_A0_300dpi.pdf (accessed 14 March 2013).

Hänel, K., and H. Reck. 2011. National Priorities of reconnecting ecosystems (Bundesweite Prioritäten zur Wiedervernetzung von Ökosystemen: Die Überwindung straßenbedingter Barrieren. Ergebnisse des F+E-Vorhabens 350782090 des Bundesamtes für Naturschutz). Naturschutz und Biologische Vielfalt 108: 1-353.

Hels, T., and E. Buchwald. 2010. The effects of road kills on amphibian populations. Biological Conservation 99: 331-340.

Herrmann, M., J. Enssle, M. Süsser, and J.-A. Krüger. 2007. Der NABU-Bundeswildwegeplan . Meckenheim: Wahrlich-Druck.

Huijser, M. P., J.W. Duffield, A.P. Clevenger, R.J. Ament, and P.T. McGowen. 2009. Cost-benefit analyses of mitigation measures aimed at reducing collisions with large ungulates in the United States and Canada; a decision support tool. Ecology and Society 14(2): 1-15.

Iuell, B., G.J. Bekker, R. Cuperus, J. Dufek, G. Fry, C. Hicks, V. Hlaváč, V.B. Keller, C. Rosell, T. Sangwine, N. Tørsløv, and B.L.M. Wandall. 2003. Wildlife and traffic: a European handbook for identifying conflicts and designing solutions . Utrecht: KNNV Publishers. 
Jaarsma, C.F., and G. Willems. 2002. Reducing habitat fragmentation by minor rural roads through traffic calming. Landscape and Urban Planning 58: 125-135.

Jaeger, J.A.G. 2000. Landscape division, splitting index, and effective mesh size: new measures of landscape fragmentation. Landscape Ecology 15: 115-130.

Jaeger, J.A.G., J. Bowman, J. Brennan, L. Fahrig, D. Bert, J. Bouchard, N. Charbonneau, K. Frank, B. Gruber, and K. Tluk von Toschanowitz. 2005a. Predicting when animal populations are at risk from roads: an interactive model of road avoidance behavior. Ecological Modeling 185: 329-348.

Jaeger, J.A.G., S. Grau, and W. Haber. 2005b. Einführung: Landschaftszerschneidung und die Folgen. Gaia 14: 98-100.

Kaseloo, P.A. 2006. Synthesis of noise effects on wildlife populations. In Proceedings of the 2005 international conference on ecology and transportation, eds. C.L. Irwin, P. Garrett and K.P. McDermott, pp. 33-35. Raleigh: Center for Transportation and the Environment.

Keller, I., and C.R. Largiader. 2003. Recent habitat fragmentation caused by major roads leads to reduction of gene flow and loss of genetic variability in ground beetles. Proceedings of the Royal Society 270: 417-423.

Keller, I., W. Nentwig, and C.R. Largiadèr. 2004. Recent habitat fragmentation due to roads can lead to significant genetic differentiation in an abundant flightless ground beetle. Molecular Ecology 13: 29832994.

Krebs, $\quad$ A. 2008. Ethics of Nature Http://www.bpb.de/gesellschaft/umwelt/bioethik/33722/naturethik (accessed on 20 February 2013)

Lassen, D. 1987. Unzerschnittene verkehrsarme Räume über $100 \mathrm{~km}^{2}$ Flächengröße in der Bundesrepublik Deutschland - Fortschreibung 1987. Natur und Landschaft 12: 532-535.

Lindenmayer, D.B. 2006. Foreword. P. 11. In Biodiversidad en Ambientes Fragmentados de Chile: Patrones y Procesos a Diferentes Escalas, eds. A.A. Grez, J.A. Simonetti and R.O. Bustamante, pp. 11-12. Santiago: Editorial Universitaria.

MA (Millennium Ecosystem Assessment). 2005. Ecosystems and Human Well-being: Synthesis . Washington, DC: Island Press.

Mader, H.J. 1979. Die Isolationswirkung von Verkehrsstraßen auf Tierpopulationen, untersucht am Beispiel von Arthropoden und Kleinsaugern der Waldbiozonöse . Bonn-Bad Godesberg: Federal Agency for Nature Conservation.

Murphy, P.R., and R.F. Poist. 2000. Green logistics strategies: an analysis of usage patterns. Transportation Journal 40(2): 5-16.

Nussbaum, M.C. 2006. Frontiers of Justice. Disability, Nationality, Species Membership . Cambridge/London: Belknap.

Oggier, P., A. Righetti, and L. Bonnard. 2007. Zerschneidung von Lebensräumen durch Verkehrsinfrastrukturen COST 341 . $2^{\text {nd }}$ edition. Bern: Bundesamt für Umwelt; Bundesamt für Raumentwicklung; Bundesamt für Verkehr \& Bundesamt für Straßen.

Reck, H., and G. Kaule. 1992. Straßen und Lebensräume - Ermittlung und Beurteilung straßenbedingter Auswirkungen auf Pflanzen, Tiere und ihre Lebensräume . Bonn: Typo-Druck.

Reijnen, R., and R. Foppen. 1994. The effects of car traffic on breeding bird populations in woodland. I. Evidence of reduced habitat quality for willow warblers (Phylloscopus trochilus) breeding close to a highway. Journal of Applied Ecology 31: 85-94.

Reijnen, R., R. Foppen, and G. Veenbaas. 1997. Disturbance by traffic of breeding birds: evaluation of the effect and considerations in planning and managing road corridors. Biodiversity and Conservation 6 : 567-581.

Riley, S.P.D, J.P. Polinger, R.M. Sauvajot, E.C. York, C. Bromley, T.K. Fuller, and R.K. Wayne. 2006. A southern California freeway is a physical and spatial barrier to gene flow in carnivores. Molecular Ecology 15: 1733-1741. 
Roedenbeck, I.A., and J.A.G. Jaeger. 2006. Auf dem Weg zu straßenökologischer Forschung auf Landschaftsebene. Naturschutz und Landschaftsplanung 38: 297-299.

Sala, E.O., F.S. Chapin III, J.J. Armesto, R. Berlow, J. Bloomfield, R. Dirzo, E. Huber-Sanwald, L.F. Huenneke, R.B. Jackson, A. Kinzig, R. Leemans, D. Lodge, H.A. Mooney, M. Oesterheld, N.L. Poff, M.T. Sykes, B.H. Walker, M. Walker, and D.H. Wall. 2000. Global Biodiversity Scenarios for the Year 2100. Science 287: 1770-1774.

Schupp, D. 2005. Umweltindikator Landschaftszerschneidung: Ein zentrales Element zur Verknüpfung von Wissenschaft und Politik. Gaia 14: 101-106.

Selva, N., S. Kreft, V. Kati, M. Schluck, B.G. Jonsson, B. Mihok, H. Okarma, and P.L. Ibisch. 2011. Roadless and Low-Traffic Areas as Conservation Targets in Europe. Environmental Management 48: 865877.

Semlitsch, R.D., T.J. Ryan, K. Hamed, M. Chatfield, B. Drehman, N. Pekarek, M. Spath, and A. Watland. 2007. Salamander Abundance along Road Edges and within Abandoned Logging Roads in Appalachian Forests. Conservation Biology 21:159-167.

Shanley, C.S., and S. Pyare. 2011. Evaluating the road-effect zone on wildlife distribution in a rural landscape. Ecological Society of America 2: 1-16.

Stoner, D. 1925. The toll of the automobile. Science 61: 56-57.

Tilman, D., R.M. May, C.L. Lehman, and M.A. Nowak. 1994. Habitat destruction and the extinction debt. Nature 371: 65-66.

Turner, J.M. 2006. Conservation science and forest service policy for roadless are-as. Conservation Biology 20: 713-722.

Van der Grift, E.A., R. Van der Ree, L. Fahrig, S. Findlay, J. Houlahan, J.A.G. Jaeger, N. Klar, L.F. Madriñan, and L. Olson. 2013. Evaluating the effectiveness of road mitigation measures. Biodiversity and Conservation 22: 425-448.

Van der Ree, R., J.A.G. Jaeger, E.A. Van der Grift, and A.P. Clevenger. 2011. Effects of roads and traffic on wildlife populations and landscape function: road ecology is moving towards larger scales. Ecology and Society 48: 1-8.

Yahner, R.H. 1998. Changes in Wildlife Communities Near Edges. Conservation Biology 2(4): 333339. 




\section{AFFIDAVIT}

"I declare on oath that the present text 'Quantification of Potential Ecological Impacts of Road Transport' was singly written by myself. My contributions to the four articles also were singly written by myself. I did use no other than the cited auxiliary means and texts. All ideas and words of other authors are indicated and referenced". 